\title{
Detecting Early Choroidal Changes Using Piecewise Rigid Image Registration and Eye-Shape Adherent Regularization
}

\author{
Inaugural dissertation \\ to \\ be awarded the degree of Dr. sc. med. \\ presented at \\ the Faculty of Medicine \\ of the University of Basel \\ by \\ Tiziano Ronchetti \\ from Bern, Switzerland
}

Basel, 2020

Original document stored on the publication server of the University of Basel edoc.unibas.ch 
Approved by the Faculty of Medicine

on application of

Prof. Dr. med. Selim Orgül, University of Basel, First examiner

Prof. Dr. Philippe C. Cattin, University of Basel, Second examiner

Dr. Christoph Jud, University of Basel, Further advisor

Prof. Dr. Dr. med. Daniel Barthelmes, University of Zürich, External expert

Dr. med. Peter M. Maloca, Institute of Molecular and Clinical Ophthalmology Basel, Further expert

Dr. Boris Považay, Bern University of Applied Sciences, Further expert

Basel, $22^{\text {nd }}$ June 2020

Prof. Dr. med. Primo Schär

Dean 
Dedicated to my mother and my sister and my beloved fiancée.

Thanks for your constant love and support.

In my father's memory. 



\section{Contents}

Acknowledgments vii

Summary $i x$

Zusammenfassung $x i$

1. Introduction $\quad 1$

1.1. Preamble: Changes in the human eye and their consequences . . . . . . . . . . 1

1.2. Motivation: Correlations between changes in the choroidal thickness and myopia 1

1.3. Contribution

$C R A R$, a regularized piecewise rigid registration approach, as a sensitive objective progress indicator for several ocular diseases and their respective treatments . . . 3

1.4. Outline: Structure of this Thesis .................... 4

2. Background $\quad 5$

2.1. Medical Background . . . . . . . . . . . . . . . . . . 5 5

2.1.1. Anatomy of the Eye . . . . . . . . . . . . . . . 5

2.1.2. Anatomy of the Choroid . . . . . . . . . . . . . . . 7

2.1.3. Myopia and Ocular Diseases . . . . . . . . . . . . . . . . . . 9

2.1.4. Choroidal and Intra-Retinal Layers: Thickness as Biomarker . . . . . . . 14

2.2. Technical Background . . . . . . . . . . . . . . . . . . 17

2.2.1. General Information about Optical Coherence Tomography . . . . . . . 18

2.2.2. Time and Frequency Domain OCT . . . . . . . . . . . . . . 20

2.2.3. Mathematical Background of FD-OCT . . . . . . . . . . . . 26

3. Mathematical Principles underlying the Regularized Piecewise Rigid Registration Approach CRAR

3.1. Image Registration . . . . . . . . . . . . . . . . . . . . . . . . . . . 29

3.1.1. General Remarks . . . . . . . . . . . . . . . . . . . . . 29

3.1.2. Block Matching as Method for Piecewise Rigid Registration . . . . . . . . 30

3.1.3. Regularization ....................... 32

3.2. The Registration Problem in Mathematical Terms . . . . . . . . . . . . . . . 33

3.2.1. The Variational Approach: Getting Low on Energy. . . . . . . . . . . . . 33

3.2.2. General Remarks . . . . . . . . . . . . . . . . . . . . . 34

3.3. Mathematical Model of CRAR . . . . . . . . . . . . . . . . . 35

3.3.1. The Main Idea . . . . . . . . . . . . . . . . . . . . . . 35

3.3.2. Piecewise Rigid Registration . . . . . . . . . . . . . . . . 35

3.3.3. Radial Differences Regularization . . . . . . . . . . . . . . . . 37 
5. Detecting Early Choroidal Changes using Piecewise Rigid Image Registration and EyeShape Adherent Regularization

5.1. B-Spline Deformation . . . . . . . . . . . . . . . . . . . 59

6. Statistical Framework for Validation without Ground Truth of Choroidal Thickness $\begin{array}{ll}\text { Changes Detection } & 60\end{array}$

6.1. Further Experiments . . . . . . . . . . . . . . . . . . 78

6.2. Supplementary Information . . . . . . . . . . . . . . . . 78

7. Feasibility Study of Subfoveal Choroidal Thickness Changes in Spectral-Domain Optical Coherence Tomography Measurements of Macular Telangiectasia Type 2

$\begin{array}{lr}\text { 8. Discussion and Conclusion } & 89\end{array}$

8.1. Achievements . . . . . . . . . . . . . . . . . . . 89

8.2. Critical Points Limitations . . . . . . . . . . . . . . . . . . . 91

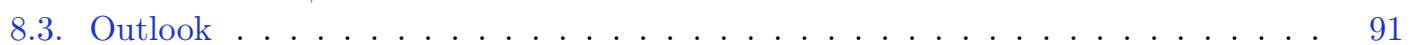

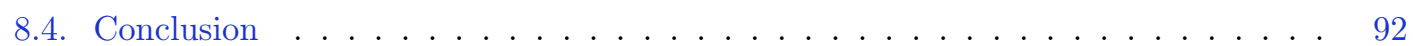

$\begin{array}{lr}\text { 9. Bibliography } & 93\end{array}$

$\begin{array}{lr}\text { List of Figures } & 106\end{array}$

$\begin{array}{lr}\text { Appendices } & 107\end{array}$

$\begin{array}{lr}\text { A. Additional Technical Information } & 109\end{array}$

A.1. Dual-Wavelength System: Hydra-Spectralis . . . . . . . . . . . . . . . . . 109

B. Additional Mathematical Information $\quad 111$

B.1. Mathematical Definitions underlying CRAR . . . . . . . . . . . . . . . 111

B.2. First Variation of $\mathcal{D}^{\mathrm{SSD}}$ and $\mathcal{D}^{\mathrm{NCC}} \ldots \ldots \ldots \ldots \ldots$

B.3. B-Splines . . . . . . . . . . . . . . . . . . . 113

C. Additional Visual Information $\quad 115$

C.1. Illustrations Technical Background . . . . . . . . . . . . . . . . . . . . . . 115

C.2. Illustrations Mathematical Background . . . . . . . . . . . . . . . . . . . 117

$\begin{array}{lr}\text { Curriculum Vitae } & 119\end{array}$ 


\section{Acknowledgments}

This thesis would not have been possible without the support and help of many people. First of all, many thanks to my supervisor, Prof. Dr. Philippe C. Cattin, for always believing in me and for giving me the opportunity to become a member of his amazing research team of the Center for medical Image Analysis and Navigation (CIAN). I have always felt very well tutored and in very good hands. I have really appreciated how everyone was always available to help the others in case of difficulties. I have also enjoyed the pleasant and motivating atmosphere in which I found the tranquility to quickly progress in my research. In particular, I thank Dr. Christoph Jud, who was my tutor at CIAN, for his great and passionate support.

I would like to thank the research team of the Institute for Human Centered Engineering (HuCE)-optoLab at the University of Applied Sciences (BUAS) in Biel for their support and assistance during this time. Special thanks go to Prof. Christoph Meier for making it possible for me to participate in this interesting SNSF project and for always giving me the support I needed. Many thanks to Dr. Boris Považay, the project leader, for his great support and experienced inputs for my papers. It was very rewarding to have the opportunity to collaborate with him. Furthermore, my gratitude goes to the staff at the Hong Kong Polytechnic University's School of Optometry, especially Dr. Rachel Ka Man Chun and Prof. Dr. Chi-Ho To, who performed the challenging long-term study with children and teenagers.

My gratitude also goes to Prof. Dr. Selim Orgül for his trust in my skills, for all the interesting discussions and for broadening my horizon in other fields of research. I am also very grateful to Dr. Maloca for giving me very important inputs for the medical eye research and for his contagious enthusiasm in taking on new challenges in unexplored areas of ophthalmology. Many thanks to both for their great support. I am also very grateful to Prof. Dr. Scholl for all the motivating discussions and his willingness and interest to cooperate with us in our future projects.

I offer my sincere gratitude to the directorate of the BUAS, especially to Dr. Herbert Binggeli (president), Dr. Lukas Rohr (director), Dr. Peter Brunner (head of the research division), Dr. Erich Wyler (head of teaching and resource planning), Pierre-André Chevalier (dean and head MNG) and Dr. Walter Businger (head of mathematics division) for offering me the opportunity to work on my Ph.D. thesis during the last four years. Many thanks to Dr. Andreas Stahel for the animated, interesting and interdisciplinary discussions.

In grateful memory to Prof. Rolf G. Müller, who initiated me to the BUAS.

Many thanks to my friend Michael Müller, who supported me very generously when I had questions in my first steps in using Python and Java. My special thanks go to my friend Jan Schippers, who although he actually does not come from the natural sciences, has already made himself available so generously for prying questions, critical thoughts and passionate discussions.

Finally, it is very important to me to thank all my colleagues of CIAN in Allschwil, especially Dr. Alina Giger, Dr. Nadia Möri, Dr. Robin Sandkühler, Dr. Stephan Wyder, Dr. Simon Pezold, Dr. Antal Horváth, Dr. Simon Andermatt, Dr. Jörg Wagner, Peter von Niederhäusern, Dr. Beat Fasel, Aydin Ürgen and Corinne Eymann-Baier. Thanks for the great support! 



\section{Summary}

Choroidal and retinal thickness changes can occur in patients with refractive errors (e.g. myopia) or ocular diseases (e.g. central serous chorioretinopathy, glaucoma, etc.) and must therefore be detected as early as possible and monitored. Image acquisition is usually done with the help of optical coherence tomography (OCT), which allows 2- and 3-dimensional images with micrometer resolution. Segmentation-based image analysis methods are used to detect and quantify thickness changes. However, segmenting the choroid is often a challenging task because of low contrast, loss of signal and the presence of artifacts in the acquired images. In particular, in vivo imaging of the choroid-sclera interface (CSI), the border separating the choroid from the sclera, is prone to these image degradations.

In this thesis, we present CRAR, a novel method for the early detection of choroidal changes based on piecewise rigid image registration. CRAR allows elastic modeling of the relatively soft choroid without affecting the more rigid properties of the surrounding sclera and retina. Rather than insisting on finding the exact position of the CSI, we focus on the changes of the entire choroid-sclera border. This enables us to circumvent the aforementioned difficulties because, using this approach, an exact recognition of the choroid-sclera boundary is not required.

In this approach, we focus on juvenile myopia (also called "school myopia"), which in Asian regions, and especially in China, has reached almost epidemic dimensions by now. Since juvenile myopia correlates with changes in the thickness of the choroid, but not with its structure as such, we restrict the transformation model to the anterior-posterior $(z-)$ direction. The proposed regularization allows respecting the eye's natural shape. In this context, the local homogeneity of the transformations in nasal-temporal $(x-)$ and superior-inferior $(y-)$ direction are boosted by penalizing their radial differences.

However, a comprehensive evaluation of the performance in detecting such changes is challenging, as a ground truth for comparison with the in vivo situation does not exist. In order to overcome this limitation, we present a statistical validation framework for automated choroidal thickness changes detection, in which a method purely based on the common agreement between the algorithm and all experts is combined with an exhaustive power analysis approach. We show the strengths of the framework with the example of CRAR: the framework demonstrates if an algorithm functions at an expert level, while the integrated power analysis allows concluding whether the algorithm performs even better than the experts.

We further applied CRAR to macular telangiectasia type 2 (MacTel2). The analysis of followup images of this disease suggests that there might be a correlation between changes in the choroidal thickness and the further development of MacTel2. The further refinement of the presented method CRAR can provide an objective and sensitive tool to analyze and monitor the progress of myopia, and beyond. 



\section{Zusammenfassung}

Änderungen der Dicke von Chorioidea (Aderhaut) und Retina (Netzhaut) können bei Patienten mit Brechungsfehlern, beispielsweise Myopie (Kurzsichtigkeit), oder Augenerkrankungen, wie z.B. Chorioretinopathia centralis serosa, Glaukom (Grüner Star), auftreten und müssen daher so früh wie möglich erkannt und überwacht werden. Die Bilderfassung erfolgt in der Regel mit Hilfe der Optischen Kohärenztomographie ("optical coherence tomography", kurz "OCT"), welche 2- und 3-dimensionale Aufnahmen in Mikrometeraufösung ermöglicht. Für die Erkennung und Quantifizierung der Änderungen werden segmentierungsbasierte Bildanalyseverfahren eingesetzt. Die Segmentierung der Chorioidea erweist sich allerdings oft als schwierig. Gründe dafür sind geringer Kontrast, Signalverlust und die Anwesenheit von Artefakten in den aufgenommenen Bildern. Die in vivo Bildgebung der Grenzschicht zwischen Chorioidea und Sklera (Lederhaut), sogenanntes "Choroid-Sclera Interface" (CSI), leidet besonders unter einem solchen Verlust an Bildinformationen.

In der vorliegenden Arbeit stellen wir CRAR vor, eine neuartige Methode zur Früherkennung von Veränderungen in der Dicke der Chorioidea, welche auf einer stückweise rigiden Bildregistrierung basiert. CRAR ermöglicht, die relativ weiche Chorioidea elastisch zu modellieren, ohne dabei die rigideren Eigenschaften der umgebenden Sklera und Retina zu beeinträchtigen. Anstatt darauf zu beharren, die genaue Position der CSI zu bestimmen, konzentrieren wir uns auf die Veränderungen des gesamten Chorioidea-Sklera-Grenzbereichs. Die Tatsache, dass sich bei diesem Ansatz eine exakte Erkennung der CSI erübrigt, ermöglicht es, die oben genannten Schwierigkeiten zu umgehen.

Bei diesem Ansatz fokussieren wir uns auf juvenile Myopie (auch "Schulmyopie" genannt), welche besonders im asiatischen Raum beinahe epidemische Ausmasse erreicht hat. Da juvenile Myopie zwar mit Veränderungen der Dicke der Chorioidea, aber nicht mit deren Struktur korreliert, schränken wir das Transformationsmodell auf die anterior-posteriore $(z-)$ Richtung ein. Die vorgeschlagene Regularisierung ermöglicht, der natürlichen Form des Auges gerecht zu werden. In diesem Sinne wird die lokale Homogenität der Transformationen in nasal-temporaler $(x-)$ und superior-inferiorer (y-) Richtung durch die Bestrafung radialer Differenzen gefördert.

Eine allumfassende Evaluierung dieser Methode zur Erkennung solcher Veränderungen ist eine anspruchsvolle Aufgabe, weil die reelle Veränderung als Referenzwert nicht existiert. Abhilfe liefert eine von uns entwickelte statistische Methodik für die Validierung der automatisierten Erkennung von Veränderungen in der Dicke der Chorioidea. Hierzu kombinieren wir das Prinzip von "gegenseitiger Übereinstimmung" ("common agreement") zwischen Algorithmus und einer Gruppe von Experten, mit einem umfangreichen Power-Analyse Ansatz. Am Beispiel von CRAR demonstrieren wir die Stärken der entwickelten Validierungsmethodik: diese überprüft, ob ein Algorithmus auf Expertenniveau performt, während die integrierte Power-Analyse Rückschlüsse daraus ziehen lässt, ob der getestete Algorithmus sogar noch besser als die Experten abschneidet. 
Ferner haben wir CRAR auch für die Untersuchung von Makulärer Teleangiektasie Typ 2 (MacTel2) angewandt. Die Analyse von Verlaufsbildern dieser Krankheit deutet auf einen Zusammenhang zwischen Veränderungen der chorioidalen Dicke und dem Fortschritt von MacTel2 hin. Die Verfeinerung der vorgestellten Methode CRAR kann ein objektives und sensitives Instrument liefern, das zur Analyse- und Überwachung des Fortschritts von Myopie und darüber hinaus verwendet werden kann. 


\section{Introduction}

\subsection{Preamble: Changes in the human eye and their consequences}

From even before the moment a child is born until the adolescence, the visual system continually undergoes physiological changes that have a crucial influence on the future quality of life. As with other organs in the body, the eyes grow and develop during this phase, adapting to their environment [1-4].

In this context, excessive axial lengthening of the eyeball can lead to negative consequences on vision, for example high myopia or, in extreme cases, pathological myopia, which impair visual function [5]. While low, up to high-grade myopia can be corrected with glasses or contact lenses, more severe nearsightedness can have serious consequences, such as retinal detachment [6], making a surgical intervention necessary to prevent blindness; see Fig. 1.1 as well as Fig. 2.6(c) and (f).

As a result of the eyeball elongation, inner ocular tissues such as the retina can become thin due to stretching $[7,8]$. The same thinning process also seems to have an impact on the choroid $[9,10]$. This is supported by several studies [10-15], which reported that shortsighted eyes could be characterized by a thinner choroid; see Fig. 2.6(b), (c), (e) and (f). The pathogenesis of these impairments is not yet fully understood.

\subsection{Motivation: Correlations between changes in the choroidal thickness and myopia}

Ocular diseases often represent not only an unpleasant disturbance of sight, but can also lead to partial, and in some cases, total loss of vision. When structural alterations in the retina are observed, irreversible changes may already have occurred, so timely intervention is crucial. It would therefore be helpful to obtain indicators for such changes in their early stages.

Researchers have found that the manifestation and progression of myopia are accompanied by changes in thickness of the choroid [16-18]. While in adults choroidal thickness decreases with advancing age in a physiological process [13, 19-21], studies assessing choroidal development during childhood and adolescence have proposed contradictory conclusions. Subfoveal choroidal thickness is found to be positively correlated with age in Caucasian children [16-18, 22], but negatively in Asian children, in whom the prevalence of myopia is significantly higher [20,23].

The choroid plays an active role in emmetropization, both by modulation of its thickness to adjust the retina to the optical focus plane (choroidal accommodation) and through the regulation of scleral growth $[24,25]$. Its complex interaction with other tissues as well as its strong dependence on many other factors such as blood pressure and diurnal variation, demand a precise 


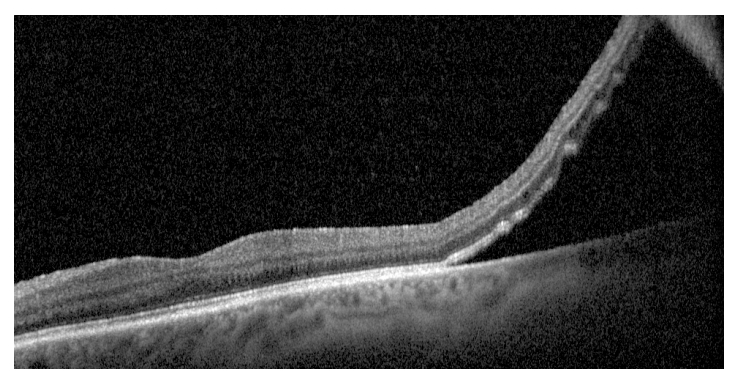

(a)

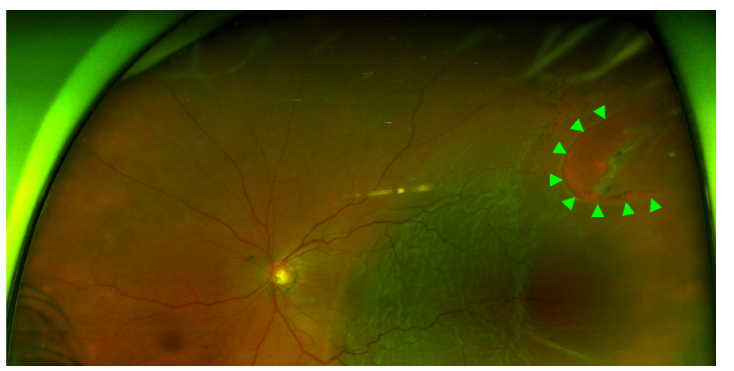

(b)

Figure 1.1.: Retinal detachment and tear.

(a) OCT B-scan of a left retina with advanced retinal detachment. (b) Wide field retinal fundus image of a myopic eye with a horseshoe-shaped retinal tear (see the area marked with the green arrows). (The images were kindly provided by the Eye Clinic at the Lindenhofspital in Bern, Switzerland.)

and reliable monitoring method [26,27]. Choroidal thickness and structure have been demonstrated to be important markers for the monitoring of myopic progression and predicting myopia, supporting the theory that choroidal abnormality could play a vital role in the pathogenesis of myopic degeneration [26, 28-30].

In the present study, our attention was focused on the development of a novel method to detect minute changes in choroidal thickness in case of myopia at its early onset. To achieve this, we focused on children and young adults (preferably aged 8-13) as a suitable study group. Because of the high prevalence of juvenile myopia [31,32], the Chinese volunteers enrolled in our study seemed to be especially interesting. Our goals were to tackle the following main challenges:

(a) Segmentation of the choroid (in particular of its outer border, the choroid-sclera interface or CSI, see Fig. 1.2) is challenging to perform due to low contrast, loss of signal and presence of artifacts in optical coherence tomography (OCT, see Sec. 2.2).

(b) The architecture of the posterior pole, including the relatively soft choroid that is surrounded by the more rigid scleral and retinal tissues (see Fig. 1.2), is so interleaved that these structures strongly suggest a locally operating, piecewise rigid approach, as proposed in [28, 33].

(c) If we developed such a method, the reliability of our results without an existing ground truth for validation would have to be demonstrated.

In this thesis, a new method called "Detecting early choroidal changes using piecewise rigid image Registration and eye-shape Adherent Regularization", in short form "CRAR", is proposed, which

(a) instead of determining the exact position of the CSI, detects its temporal displacement, so we can bypass the aforementioned limitations in OCT images;

(b) subdivides the global non-rigid matching problem into numerous local rigid registrations of the individual subregions. In this way, the soft choroid is elastically deformed and the more rigid characteristics of the surrounding sclera and retina are preserved. The incorporated 


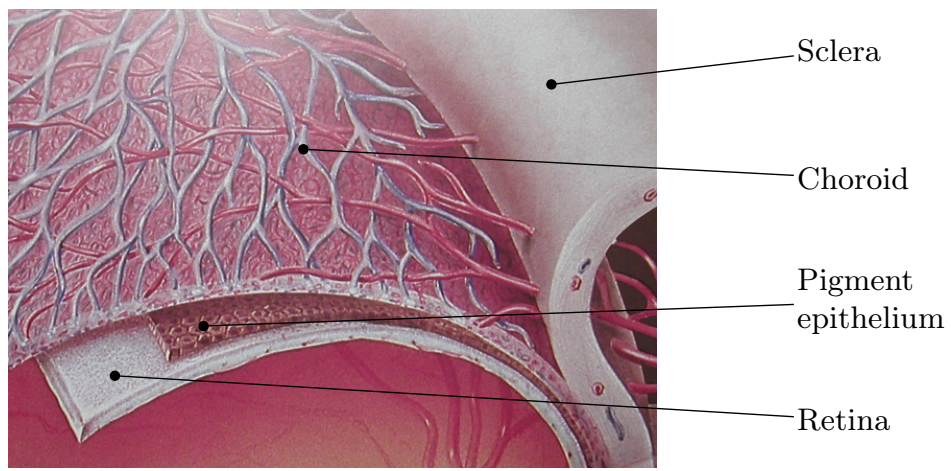

Figure 1.2.: Interleaved nature of the human eye (source: [34], public domain).

2D radial cubic B-spline kernel guarantees a regularization of the displacement field that is adherent to an eye's natural shape;

(c) overcomes the lack of a real ground truth with the support of its built-in statistical validation framework for automated detection of choroidal thickness changes, which combines a method purely based on common agreement with an exhaustive power analysis approach.

\subsection{Contribution}

\section{$C R A R$, a regularized piecewise rigid registration approach, as a sensitive objective progress indicator for several ocular diseases and their respective treatments}

To verify the feasibility of such a piecewise operating registration approach, we first propose the simplified block matching-based prototype "ICR" (short for "Intensity-based Choroidal Registration"), which processes only scan-wise and in which the regularization is only integrated as a post-processing step. In the second step, we expand the method into 3D. By optimizing the regularization using a multiresolution matching procedure, mismatches are corrected at an earlier stage leading to an improvement in the detection precision of $100 \%$. In addition, by using a regularizer with a compactly supported kernel we guarantee a local homogeneity of the displacements and a uniform smoothness of the results, matching the anatomic structure of the eye much better than in ICR. In the third step, in absence of a real ground truth for comparison of the results, we develop a statistical validation framework to evaluate the performance in the automatic detection of changes in choroidal thickness.

Summarizing, the contribution of this thesis is manifold: (1) We propose a novel, efficient, accurate and fully automated 3D registration algorithm for the detection of temporal choroidal thickness changes. (2) By the decomposition of the global non-rigid registration problem into numerous local rigid registrations, we create a deformation model that elastically deforms the soft choroid and preserves the more rigid characteristics of the surrounding sclera and retina. (3) We create a kernel-based framework allowing an eye-shape adherent regularization. (4) We present a statistical framework to validate the detection of choroidal thickness changes, that 


\section{Introduction}

works even though a ground truth for comparison is not available. Furthermore, we extend the commonly used power analysis approach by leave-one-out cross validation to become an ideal component of our statistical framework. (5) Based on a clinical study in cooperation with the Hong Kong Polytechnic University and School of Optometry, we analyze OCT Bscans of volunteers with a high prevalence of juvenile myopia. Moreover, we gain insight into choroidal thickness changes pattern. (6) In a retrospective, observational study, conducted in cooperation with the Moorfields Eye Hospital, London, UK, we use our method to examine a possible correlation between choroidal thickness change and the progression of idiopathic macular telangiectasia type 2 (MacTel2).

\subsection{Outline: Structure of this Thesis}

In Chapter 1, we introduce the goals of our work and explain its relevance in relation to the prevention and treatment of ocular diseases. We explore relevant medical background in Chapter 2, Sec. 2.1. First, we provide an introduction to the anatomy of the eye, and especially the choroid, on which we focus in our study. Second, we provide a brief overview of the most prevalent eye diseases, with particular attention on myopia at its early onset, also called juvenile or school myopia, which occurs between 9 and 11 years of age with progression throughout the early teenage years $[12,28,31,33]$.

In Sec. 2.2 we offer a brief overview of the technical background underlying this research, with a focus on the basic principles of Frequency domain (FD)-OCT, which was relied upon for image acquisition in this study. A description of the developed OCT dual-wavelength device can be found in Appendix A.1.

In Chapter 3, the mathematical principles and machine learning tools which play an essential role in this thesis are presented.

Chapters 4, 5, 6, and 7 contain the accepted publications. The first enclosed publication introduces a baseline of our method with a first piecewise rigid registration-based approach, operating only slicewise in 2D. In the second publication, presented in Chapter 5 , an improved registration framework that extends the matching into 3D and introduces a regularization which adheres to the eye's shape, is proposed. In the third publication, see Chapter 6 , we present a statistical validation framework to evaluate the performance of any algorithm in detecting choroidal thickness changes, if a ground truth for comparison is not available. As an example of an automatic detection method we use the proposed registration-based method CRAR [28]. In the fourth publication, presented in Chapter 7, we investigate the correlation between changes in the thickness of the choroid and the progression of MacTel2. Finally, in Chapter 8, we present our final thoughts and ideas for future research. 


\section{Background}

In this chapter, we provide an overview of the anatomy and function of the human eye, with a focus on the choroid and its relevance. We then review some of the more prevalent ocular disorders and diseases, particularly myopia. Finally, we offer a brief overview of the technical background used for this study, especially the role of OCT.

\subsection{Medical Background}

In the Atlas of Ophthalmology [35], EyeWiki [36], and Wikipedia [37], detailed information about the human eye, as well as ocular diseases and their treatments, is provided. Information from these websites was used to write this chapter, and these can be recommended for further reading.

The International Statistical Classification of Diseases and Related Health Problems 11th Revision (ICD-11) [38], a medical coding scheme created by the World Health Organization for the documentation of diseases in general, as well as their diagnoses and symptoms, is also very recommendable. This sizeable classification comprises 12 chapters. Some of the diseases listed are merely unpleasant disorders, while others can have serious health consequences. We refer the interested reader to these works.

\subsubsection{Anatomy of the Eye}

The eye is one of the most complex organs in the human body. Figure 2.1 illustrates a healthy human eye with labels for the most important structures. The eye is divided into two segments: the anterior and the posterior segment. While the anterior segment is composed of the anterior and posterior chamber, the vitreous is part of the posterior segment and represents the largest chamber in the eye, comprising up to $80 \%$ of the eye's volume. It is filled with a transparent, jelly-like liquid, the vitreous body. The anterior chamber is located between the cornea and iris, whereas the posterior chamber is found between the iris and the lens, see Fig. 2.2. Both chambers are filled with aqueous humor, a transparent fluid. ${ }^{1}$ This fluid, consisting of $0.1 \%$ nutrient (sugar, vitamins, and proteins) and $99.9 \%$ of water, plays an essential role in the health of the eye. It supplies nourishment to the cornea and lens, maintains intraocular pressure, and gives the eye its shape. The eyeball, also called globe or bulbus oculi, is located in the orbit, where the remaining space is occupied by eyes muscles, fat, blood vessels, and nerves. Three main layers (ocular tunicas) define the globe:

- The sclera is the white, opaque, outermost layer of the eye. It is visible peripheral to the iris and is covered by the conjunctiva in the anterior part of the eye. It encompasses the back of the eye and the optic disc, or optic nerve head, the circular area on the retina

\footnotetext{
${ }^{1}$ Please note that in ophthalmology the word "humor" (from the Latin "umor") describes the bodily secretions affecting a person's health.
} 


\section{Background}

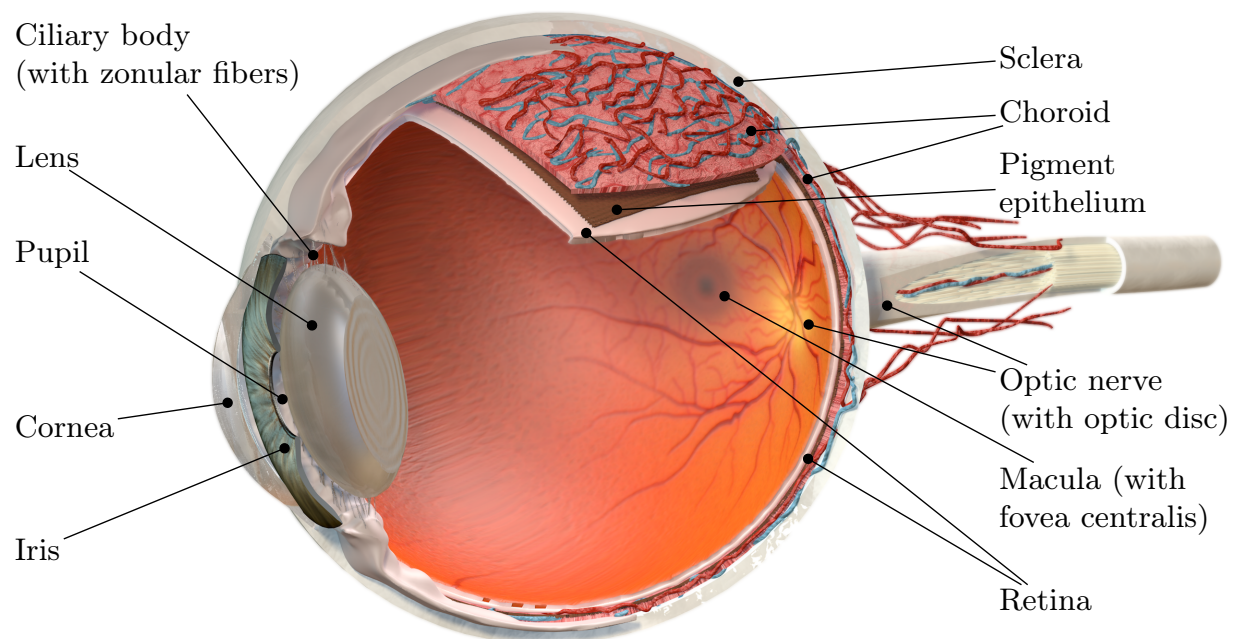

Figure 2.1.: Anatomy of the human eye (inspired by [34]).

where the optic nerve enters the eye. Consisting of thick connective tissue, the sclera forms the supporting wall of the eye, provides stable mechanical support to vulnerable internal ocular structures (such as the retina and optic nerve head) and maintains the shape of the eyeball. ${ }^{2}$ Its opacity protects against off-axial light transmission that could otherwise lead to a degeneration of the retinal image. Due to its ability to resist stress or deformation imposed by external (e.g. trauma) or internal factors (e.g. fluctuations in intraocular pressure), the sclera plays a key role in maintaining the ocular stability even under conditions that could negatively affect vision. It is slightly less curved than the transparent cornea, which covers the front part of the eye over the iris. Cornea and sclera are responsible for accurately focusing the light onto the retina and form the outer fibrous tunic of the eye bulb (tunica externa bulbi).

- The uvea (tunica media bulbi) is the middle layer of the eye and consists of the iris, the colored part of the eye, the choroid, and the ciliary body. The latter includes the muscles responsible for the shape changes of the lens and is also responsible for the production of the aqueous humor.

- The retina (tunica interna bulbi) is the innermost layer of the eye and consists of specialized, light-sensitive cells. Among others, there are two types of cells, the rods and cones. Rods process light in low-light conditions, while cones process light in well-lit conditions and are able to detect color. Light rays that have been focused by the cornea and lens eventually reach the retina which, in turn, translates the light-ray activity ("picture") into neuronal information, which is sent via the optic nerve to the brain for decoding.

\section{From Light to Vision}

Put in highly simplified terms, the human eye can be considered as a photo-sensitive system, which guides light rays reflected off an object to form an image on a sensor that we perceive as

\footnotetext{
${ }^{2}$ The word "sclera" is derived from Greek "skleros" meaning "hard".
} 


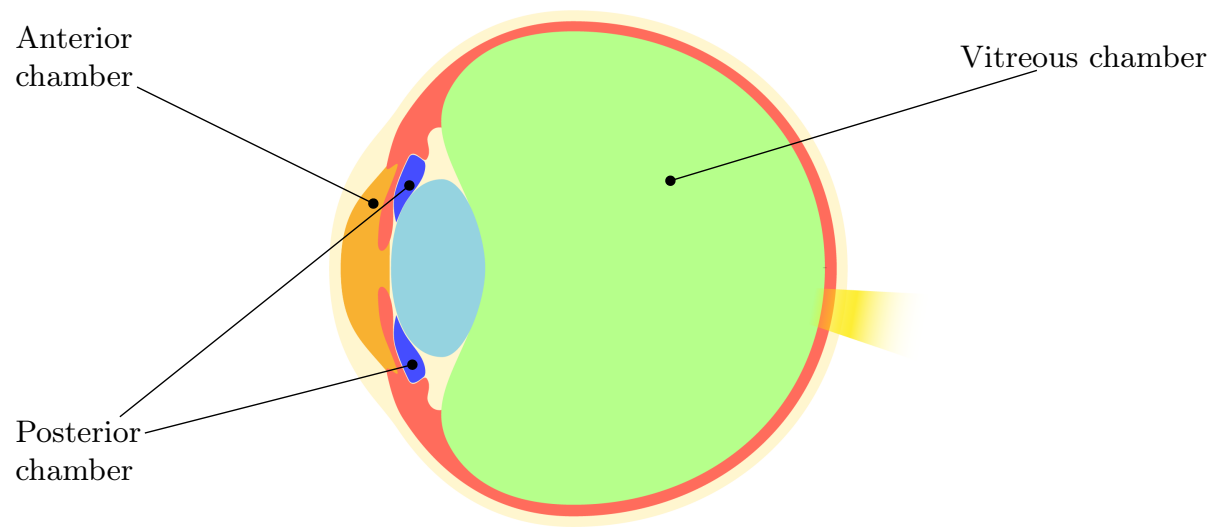

Figure 2.2.: The three chambers of the human eye.

The anterior (orange), the posterior (dark blue) and the vitreous chamber (green).

electrical signals in real time. As with the diaphragm of a camera, the iris controls the amount of light that reaches the back of the eye by adapting the size of the pupil. In low-light environments, the pupil is dilated to allow more light into the eye.

Behind the pupil the crystalline lens is located, which, in cooperation with the cornea, bundles the incident light to form an image on the retina and thus helps the eye to focus on near and far objects. In other words, the lens can change its thickness and curvature to focus on objects at different distances and to ensure that the image is as crisp as possible. The neurons of the retina are arranged in three main layers: the deepest consists of photoreceptors, which, similar to the electronic image sensor of a digital camera, convert light-ray information into nerve impulses. These impulses are then transmitted to a layer of bipolar neurons, and then to a third one that consists of so-called ganglion neurons. The optic nerve, a bundle of more than one million nerve fibers, finally transmits these impulses from the ganglion layer to the visual cortex, the part of the brain responsible for our sense of sight $[39,40]$.

\subsubsection{Anatomy of the Choroid}

The choroid, also known as choroidea or choroid tunic coat, is the vascular layer of the eye. ${ }^{3}$ It contains connective tissues and is positioned between the retina and the sclera. The thickness of the choroid varies. It is thickest at the posterior pole (about $0.22 \mathrm{~mm}$ ) and narrows down to $0.1 \mathrm{~mm}$ in the peripheral part of the eye. At birth, the human choroid is about $200 \mu \mathrm{m}$ thick, decreasing to approximately $80 \mu \mathrm{m}$ by the age of 90 years [41].

The structure of the choroid is divided into three layers. From the furthest from the retina to the closest, these are as follows [42]: Haller's layer, the outermost layer of the choroid, consists of larger blood vessels, with an average thickness of $28.2 \mu \mathrm{m}$ and $37.1 \mu \mathrm{m}$ for arteries and veins, respectively. Sattler's layer is formed of medium size blood vessels that feed and drain the choriocapillaris. The choriocapillaris is a network of large capillaries of 40-60 $\mu \mathrm{m}$ diameter supplying the retinal pigment epithelium (RPE) and the neighboring outer retina with oxygen,

\footnotetext{
${ }^{3}$ The word "choroid" is the result of the union of "chorion" and "-oid". Chorion is the Latinized form of Greek "khorion", i.e. the outermost membrane surrounding an embryo of a reptile, bird, or mammal. In mammals it contributes to the formation of the placenta. The ending "-oid" stems from "eidos" meaning "resemblance".
} 


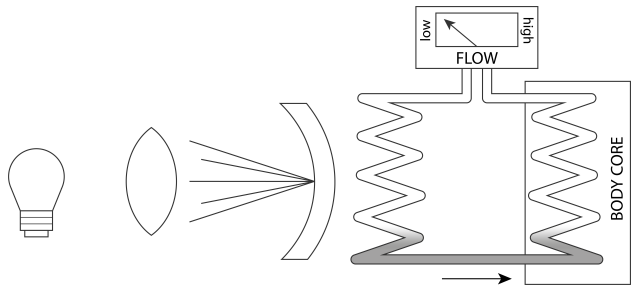

(a)

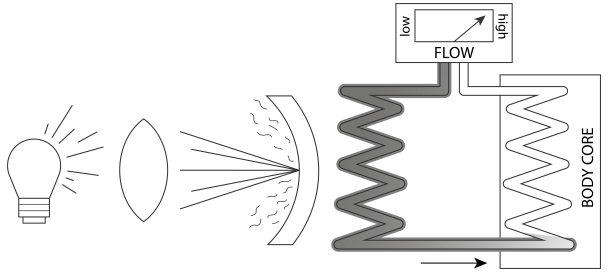

(b)

Figure 2.3.: Choroidal blood flow temperature regulation.

(a) Under normal circumstances, warmth is absorbed by the coiled circulation, stabilizing local tissue temperature. (b) In case of an increase in temperature, blood vessel walls thicken; because less warmth can be disposed of, tissue temperature rises. (Figure adapted from [43].)

micronutrients, ions, and water. When the choriocapillaris does not provide sufficient perfusion, the RPE and the outer retina suffer metabolic stress and hypoxemia, that is, an abnormal deficiency of oxygen in arterial blood. Bruch's membrane (BM), also called lamina basalis, is the 2-4 $\mathrm{mm}$ thick membrane on top of the innermost part of the choroid, forming the external surface of the RPE.

As far as it has been established, the choroid has at least the following three main functions: (1) it regulates the temperature of the retina; (2) by changing its thickness and perfusion, it adjusts the position of the retina; (3) it is responsible for the secretion of growth factors. This third function may play an important role in emmetropization, i.e. the adjustment of the eye's shape caused by general body growth, and thus in the correction of myopia or hyperopia [26]. These main functions will be laid out in more detail underneath.

\section{Function of the Choroid}

One of the choroid's primary physiologic tasks is to provide the outer retina with oxygen, blood and metabolites [26]. Thanks to the dark-colored melanin pigment in the choroid, light is absorbed, and reflections caused by scattering, which could lead to a degradation of vision, are reduced. At the same time, choroidal blood flow helps maintain a stable temperature environment for the outer-retinal layers, particularly in the macular area [43], see Fig. 2.3.

The choroid plays an active role in emmetropization, both by modulation of its thickness to adjust the retina to the optical focus plane (i.e. choroidal accommodation) and through the regulation of scleral growth $[24,25]$. Its complex interaction with other tissues and its strong dependence on many other factors, such as blood pressure and diurnal variations, demand a precise and reliable monitoring method [26,27]. When the choroid is affected, for example by degenerative changes or choroidal neovascularization, the macula and optic nerve may suffer and, therefore, a severe decrease in vision and sometimes even total blindness can occur [26]. With regard to the modulation of ocular elongation in response to defocus, short-term exposure to myopic defocus induces relative choroidal thickening, whereas hyperopic defocus leads to a thinning of the choroid [24]. This reaction of the choroid could assist in measuring retinal response to optical defocus in myopia. 


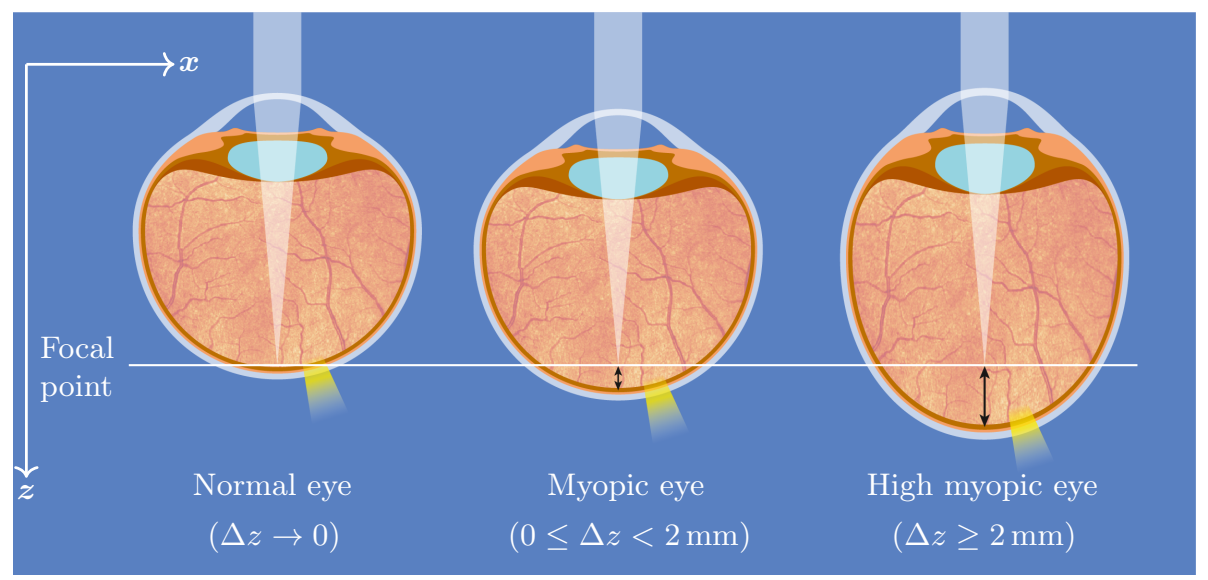

Figure 2.4.: Nearsightedness due to eye elongation.

Left: In a healthy eye, light is bundled to a spot on the retina. Middle and right: Due to the eye elongation, the light beam is focused in front of the retina, causing blurred vision. Depending on the degree of its axial lengthening $\Delta z$, an eye is classified as "myopic" $(\Delta z<2 \mathrm{~mm})$ or "high myopic" ( $\Delta z \geq 2 \mathrm{~mm}$ ). (Figure adapted from [48].)

\subsubsection{Myopia and Ocular Diseases}

\section{Myopia}

In contrast to emmetropia, the ideal state of vision providing a sharp image, refractive errors exist, such as hyperopia (also referred to as "farsightedness" [44]) and myopia (or "nearsightedness" [45]). ${ }^{4}$ Myopia can appear during childhood and gradually increase, reaching its peak when the affected individual is around 20 years old and normally stabilizing within the following ten years [47]. Myopia is the most common refractive error of the eye usually manifested by a disproportional growth of the eyeball's axial length ${ }^{5}$, see Fig. 2.4. If the axial lengthening of the eyeball overstretches the refractive power of the cornea and lens, the light beam does not reach the retina as it would in an emmetropic eye. The origin usually lies in childhood and can be seen as a "construction weakness" of the eye: the eyeball is too long, or the lens and cornea refract the light too strongly.

Generally, the refractive power of the cornea and lens is precisely adjusted to the axial lengthening of the eyeball. Light rays from nearby objects diverge and require more refraction to bring them to a focus. The eye adapts ("accommodates") to near vision by tightening the ciliary muscles and adapting the shape of the eye's lens, which becomes even rounder to allow the required refraction. This process is called "accommodation". Light rays from distant objects are nearly parallel and therefore need less refraction for focusing. The lens has its maximum focal length for distance vision when the eye is relaxed and the interior lens is the least rounded.

Figure 2.5 shows what happens if myopia manifests itself. In close vicinity, due to accommodation by the lens, objects still appear sharp, see Fig. 2.5(a) and (b), but objects in the distance are

\footnotetext{
${ }^{4}$ The word "myopia" (from the Greek "myein" meaning "to close", and "-ops" meaning "eye") probably refers to nearsighted individuals who tend to squint to better see objects in the distance [46].

${ }^{5}$ The axial length is the distance between the anterior surface of the cornea and the fovea. In healthy eyes it usually does not exceed $24 \mathrm{~mm}$.
} 


\section{Background}

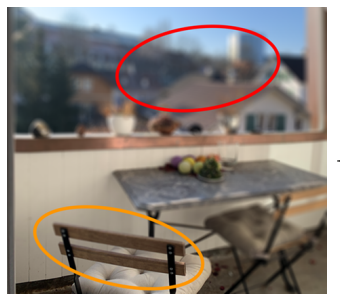

(a)

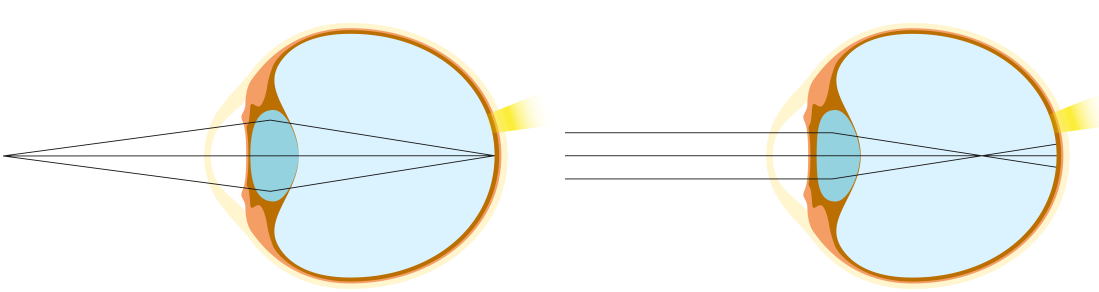

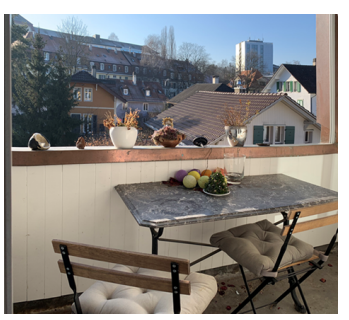

(d) (c)

(b)

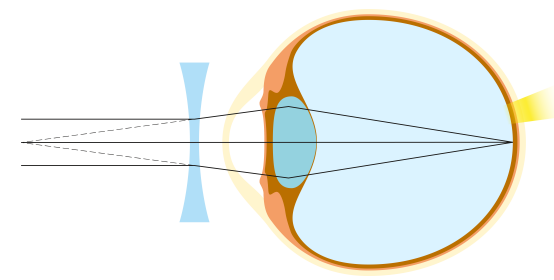

(e)

Figure 2.5.: Optics of the myopic eye.

Above: (a) In nearsighted vision close objects can be clearly seen (orange oval), whereas those in the distance are blurred (red oval). (b) Rays from close distances can be focused on the retina through accommodation of the internal (crystalline) lens. (c) Due to eye elongation, the light beams from afar converge in front of the retina, which causes blurred vision in the distance. Below: (d) Normal vision after compensating for myopia, using a corrective external lens (glasses or contact lenses). Both objects in the distance and nearby are sharp. (e) Since in a myopic eye the lens accommodation for distance vision does not adequately function, the use of an external lens is necessary for sharp vision near and far. (The defocus blur in (a) was generated by convolving the image with a disk-shaped uniform kernel.)

blurred, see Fig. 2.5(a) and (c). As a result, the focal point lies in front of the retina and vision in the distance becomes blurred. By using a corrective lens, the focal point of the light beam is perfectly located on the retina, so that each of its visual and sensory cells optimally supports the perception of the environment. Under these circumstances, the retina sends a sharp image of the surroundings to the brain and optimal visual acuity is ensured, see Fig. 2.5(d) and (e).

The unit diopter (abbreviation $d p t, 1 \mathrm{dpt}=1 \mathrm{~m}^{-1}$ ) is used as a measure of ocular refractive power and corresponds to the inverted focal length of the lens required for correction. Since nearsighted people suffer from too high refractive power, they need glasses to reduce the overall refractive power. Their correction therefore carries a minus sign, while farsighted people, who suffer from insufficient refractive power, have glasses with a positive refractive power correction. ${ }^{6}$ Myopia is graded as follows: mild, or low, up to $-3.0 \mathrm{dpt}$, moderate from $-3.0 \mathrm{dpt}$ to $-6.0 \mathrm{dpt}$, and high from - $6.0 \mathrm{dpt}$ upwards. In the absence of morphological problems the refractive consequences of myopia can be corrected with glasses or contact lenses.

\footnotetext{
${ }^{6} \mathrm{~A}$ corrective lens with a refractive power of $5 \mathrm{dpt}$ means that this is a (converging) lens with a focal length of $0.2 \mathrm{~m}$. In other words, it brings parallel rays of light to a focus of $0.2 \mathrm{~m}$ beyond the lens. Likewise, a (diverging) lens of $-2.0 \mathrm{dpt}$ has a focal length of $-0.5 \mathrm{~m}$. As a result, its focal point lies $0.5 \mathrm{~m}$ away from the lens itself.
} 


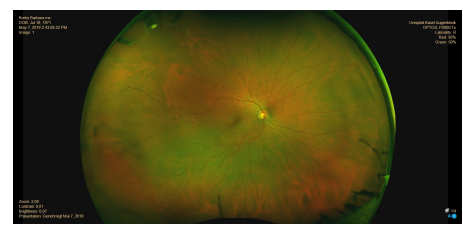

(a)

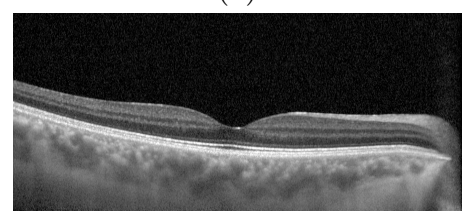

(d)

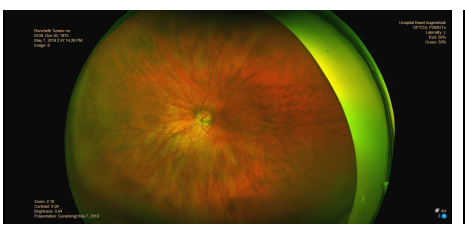

(b)

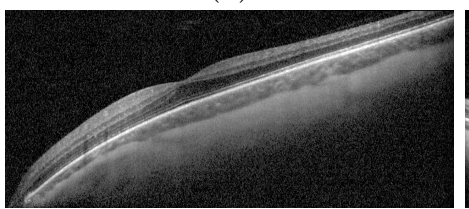

(e)

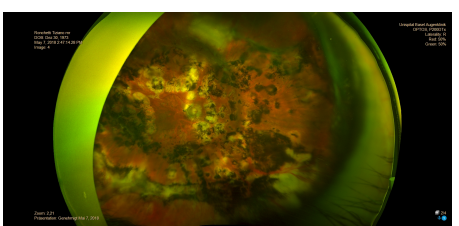

(c)

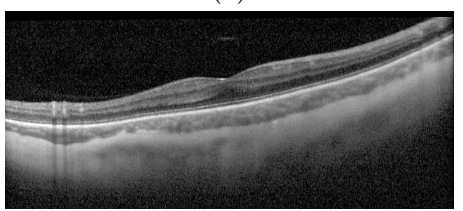

(f)

Figure 2.6.: Wide field retinal fundus (SLO) and OCT images.

Above: Wide field retinal fundus image (SLO) of (a) a normal non-myopic right eye, (b) a high myopic left eye, and (c) a right eye which underwent several surgical interventions due to retinal detachment as a consequence of pathological myopia. Below: OCT B-scans of the same three eyes, i.e. (d) a healthy right eye, (e) a high myopic left eye, and (f) an operated right eye. (c) and (f) illustrate an example of possible consequences of retinal detachment and the subsequent laser treatment, which, while ensuring the positioning of the retina, have caused scarring and partial loss of vision. (The images were kindly provided by the Eye Clinic at the University Hospital in Basel, Switzerland.)

In most cases, nearsightedness is a benign dysfunction which poses little risk to the health of the eye. However, since the most significant cause of myopia is an increase in the axial length of the eye, the retina must be stretched to cover the increased surface area. As a result, the retina in high myopic patients can become very thin [10,13-15], which, in comparison to non-myopic people, means a higher risk to develop holes, tears or even a retinal detachment, leading to rapid loss of vision $[5,6]$.

In comparison to the well-known methods which deal with the effects of myopia, for example laser eye surgery $[49,50]$, current research focuses on slowing or preventing myopic progression. Characterization of changes in the optical system and the anatomical structures of the human eye could lead to new insights into how to regulate eye growth and tackle myopia in a preventive way. A number of recent studies [51-53] have indicated that with the help of special corrective lenses, progression of myopia might be slowed during childhood so that stronger glasses are not required later. The ultimate aim is to improve eyesight and reduce dependence on glasses and contact lenses without having to undergo eye surgery.

Among the emerging clinical treatments for controlling myopia, orthokeratology (also called ortho-K) $[54,55]$ is a frequently used method for corneal reshaping. Specially designed gaspermeable contact lenses are worn overnight to correct refractive errors (primarily nearsightedness, but also astigmatism and hyperopia, and in some cases presbyopia) or to slow the progression of childhood myopia.

Causes of Myopia

Several studies have reported that genetic influences for juvenile myopia are minor [12,31]. More 


\section{Background}

important are factors which can be attributed to modern lifestyle: the increased intensity of education and less time spent outdoors. Studies have claimed that non-myopic children have a significantly reduced risk of myopia onset if they spend more time outdoors, and train to regularly focus their eyes on objects at different distances [56]. This is also true later, in cases where the development of myopia is in its early stages [57].

In Asian urban regions, the incidence rate of myopia has increased over the last 20-30 years, and around 80-90\% of students suffer from myopia [45, 58-60]. (As reported in [32], Asians, in particular Japanese, Chinese, and Korean people have a high genetic prevalence of especially high myopia.) In comparison, the increase in the Western world is slower [45]. However, and unfortunately, it is expected that myopia will likewise become widespread in coming years due to increased time spent on indoor activities that require the eyes to focus on objects close by, such as televisions, mobile phones, and computer screens [31,60].

The high prevalence of myopia makes a more than threefold increase in related visual loss over the next 30 years worldwide more than probable [58]. As mentioned previously, nearsightedness can also be caused by the cornea and/or the lens being too curved for the length of the eyeball. In some cases, myopia is due to a combination of these factors $[11,30]$.

\section{Pathological Myopia}

Approximately 1\%-3\% of the world's population suffers from a particularly severe form of shortsightedness: pathological, or malignant, myopia [61,62]. The prevalence of pathological myopiarelated visual impairment has been reported as $0.1 \%-0.5 \%$ in European studies and $0.2 \%-1.4 \%$ in Asian studies [63]. This disease is always the result of excessive axial length growth in the eyeball, and not of the strength of the refractive power of cornea and lens.

Pathological or degenerative myopia is defined as high myopia from $26.5 \mathrm{~mm}$ axial length upwards in combination with any myopia-specific pathology in the posterior part of the eye due to axial elongation. High myopic eyes have an increased risk to develop (1) stretched areas, which could lead to tears in the central retina, (2) serious bulging of the posterior part of the eye, called staphyloma, and (3) choroidal neovascularization (CNV) [15,64-67], see Fig. 2.7. When thinning and an ongoing loss of cells (atrophy) [68] occur in the macular region, central vision may gradually deteriorate.

Retinal detachment is a severe eye condition that occurs when the sensory retina is separated from the RPE [6,69], see Fig. 2.7. Tears usually occur as a result of vitreous traction to an area of vitreoretinal adhesion. Horseshoe-shaped tears (also "flap" or "U-shaped" tears, [70]) are the result of vitreous traction in combination with full thickness tears in the neurosensory retina, see Fig. 1.1. For both sexes they are more common in middle age and appear most often near the equator of the eye. The visual symptoms that indicate a potential retinal detachment include floaters, which appear like spider webs floating in the field of vision, and flashes of light, see [71,72]. Tears in myopic eyes require immediate treatment to avoid severe vision loss and even blindness, as illustrated in Fig. 2.6(c) and (f). Such treatment attempts to create a chorioretinal scar around the tear to prevent further vitreous fluid from entering the subretinal space [73].

Other complications caused by the disproportionated elongation of the eyeballs, and the directly associated stretching and thinning of the retina and sclera, can include cataract formation (a clouding of the lens [74]), choroidal neovascularization (CNV, the creation of new blood vessels derived from the choroid [75]), submacular hemorrhage (an accumulation of blood between the RPE and the neurosensory retina at the macula [76]), and posterior staphyloma (an abnormal 


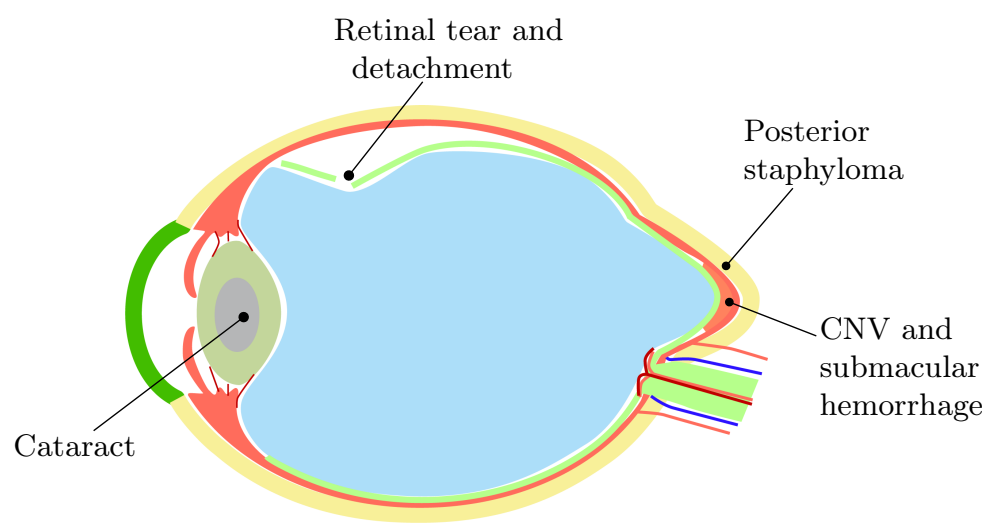

Figure 2.7.: Retinal detachment as a consequence of pathological myopia.

The retina separates from the choroid. Other complications caused by the disproportionate elongation of the eyeballs, and the directly associated stretching and thinning of the retina and sclera, can include a complicated cataract, choroidal neovascularization $(\mathrm{CNV})$, submacular hemorrhage, and the bulging of the posterior part of the eye, called staphyloma. (Figure adapted from [48].)

protuberance of the uveo-scleral tissue resulting from weakening of the cornea or sclera). Once pathological myopia has manifested itself, the best-corrected visual acuity is definitely lost $[8]{ }^{7}$

\section{Glaucoma}

Glaucoma [77] often manifests itself as an increased intraocular pressure (IOP), which causes damage to the optic nerve with no signs or symptoms in early stages. It has been suggested that myopic people may have a higher incidence of glaucoma or a higher sensitivity to increased IOP [78]. If untreated, glaucoma can lead to loss of peripheral vision and eventually to blindness. Although there is no cure for glaucoma, medications and surgery that can halt further loss of vision are available: pressure-lowering eye drops and surgery that increases fluid drainage from the eye, reducing and stabilizing IOP [79].

\section{Macular Telangiectasia}

Macular telangiectasia type 2 (MacTel2) [81] is a disease of the retina leading to a gradual deterioration only of central vision. Both eyes are involved, although not necessarily equally. It affects a well-defined oval-shaped area centered in the fovea, which is contained within the macula, the central area of the retina. Typically, there are no noticeable symptoms in the early stages. As the disease progresses, blurring, distorted vision, and a complete loss of central vision can occur within a period of 10 to 20 years. The development affects mostly people aged between 40 and 50 years. It is sometimes mistaken for age-related macular degeneration (AMD) [82] because of similar patterns of neovascularization. The cause is still unclear. Due to the lack of early symptoms and an adequate therapy for MacTel2, regular eye exams are recommended.

MacTel2 is diagnosed with the assistance of OCT [83] and specified in detail by the use of fluorescein angiography (FA) in cases with CNV [81]. No cure for MacTel2 has been found, yet.

${ }^{7}$ Best-corrected visual acuity means the best distance vision which still can be achieved using eyeglasses or contact lenses. 


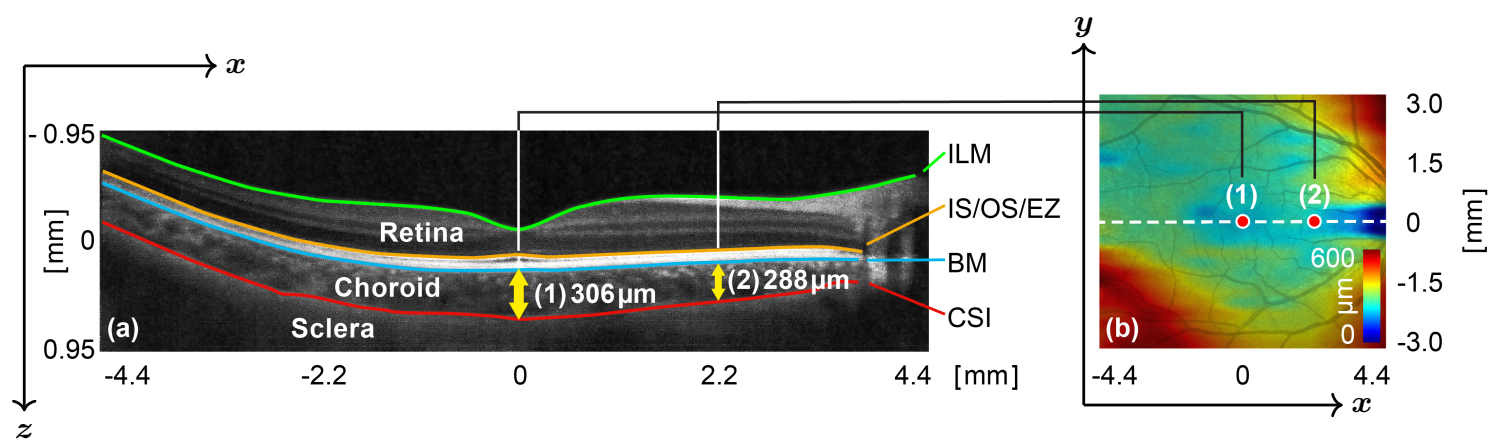

Figure 2.8.: Commonly used representations of the thickness of the choroid.

(a) OCT B-scan with segmented layers corresponding to the white dotted line in (b). Inner limiting membrane (ILM), Bruch's membrane (BM) and choroid-sclera interface (CSI). IS/OS/EZ denote the inner segment/outer segment/ellipsoid zone. (b) A color-coded choroidal thickness map overlying the fundus image. The indicated locations (1) and (2) correspond to the central and paracentral choroidal thickness measurements (1) and (2) in (a) (source: [80]).

However, in the case of neovascular vessel growth, the injection of anti-vascular growth agents (anti-VEGF) has been effective [84]. In Chapter 7 we shed light on the pathogenesis of MacTel2 by applying our method, CRAR, on subjects with the disease, to detect early changes in the thickness of the choroid.

\subsubsection{Choroidal and Intra-Retinal Layers: Thickness as Biomarker}

According to $[85,86]$ a biomarker is defined as a characteristic that is objectively measured and evaluated as an indicator of normal biological processes, pathogenic processes, or pharmacological responses to a therapeutic intervention. Biomarkers are of increasingly high importance in the realm of "personalized" medicine, because they are valuable for prognosis, treatment selection and the detection of therapeutic responses.

\section{Choroidal Thickness as Biomarker}

In the literature, for example [87], choroidal thickness is commonly defined as the Euclidean distance along the A-scans/in z-direction between the posterior edge of the hyper-reflective RPE, represented by BM, and the posterior surface of the choroid, delimited by the CSI, see Fig. 2.8. It is an important biomarker for myopia [26, 28-30] and other pathologies such as age-related macular degeneration (AMD) [82,88] and diabetic macular edema (DME) [89].

On the other hand, studies have suggested that there is no major association between glaucoma and macular choroidal thickness [90]. Therefore, detecting and monitoring small changes in the choroid could deliver insight into the pathogenesis of such diseases and assist in the planning of their treatment. If the image plane is perpendicular to BM and the CSI, measuring the choroidal thickness along the A-scan direction, as defined above, corresponds to the factual, biological thickness of the choroid.

However, this perpendicular position only occurs in an ideal scenario, and even a small malpositioning of the scanner's nodal point is sufficient to produce a difference between the measured and 


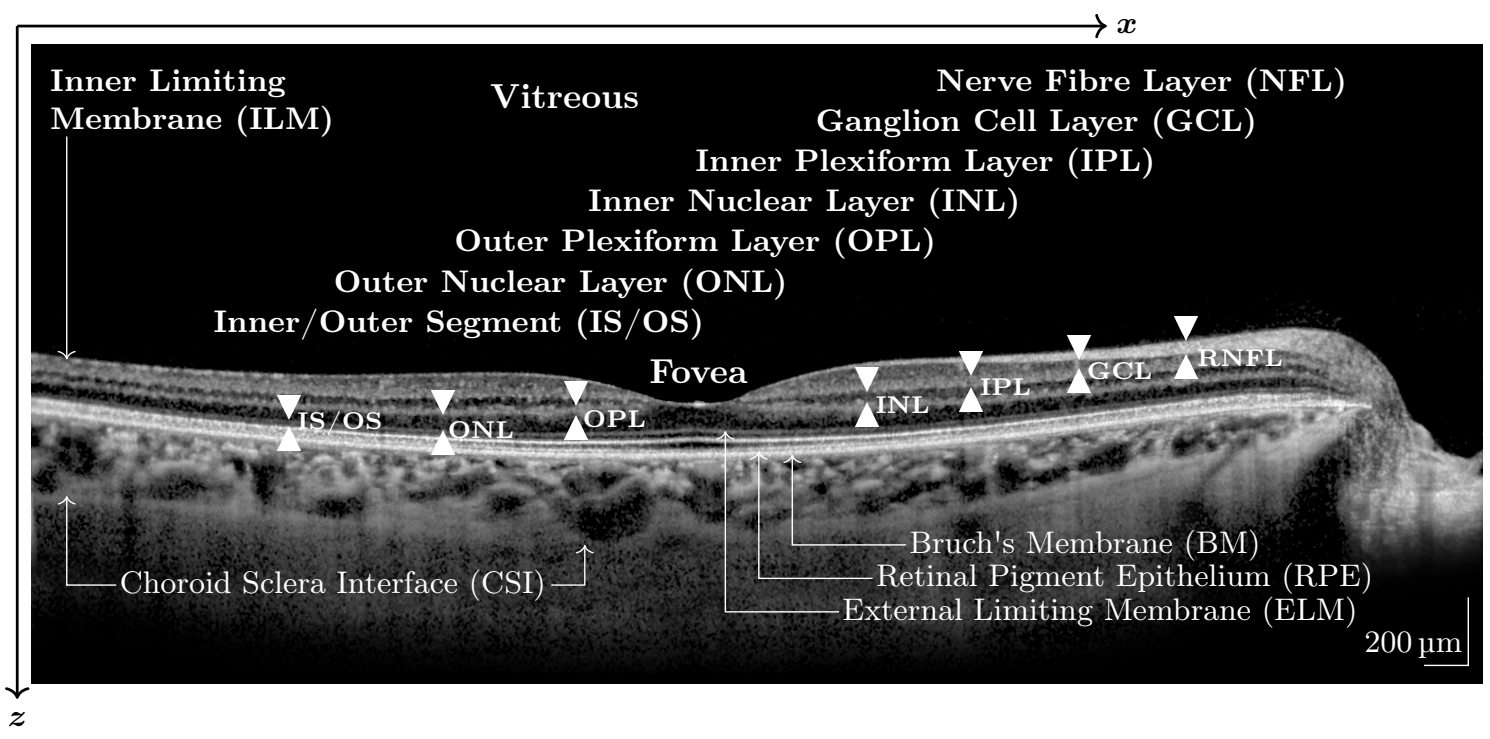

Figure 2.9.: The intra- and outer-retinal boundaries.

The intra- (ILM, NFL, GCL, IPL, INL, OPL, IS/OS) and outer- (ELM, BM, RPE, CSI) retinal boundaries. The intra-retinal layer located between the ILM and NFL is denoted by RNFL (retinal nerve fiber layer). The area between the inner and outer segment photoreceptors is also called the ellipsoid zone. In the other cases, the intraretinal layer is referred to in the same way as its lower boundary. For example, the layer between the IPL (the upper boundary) and the INL (the lower one) is called the INL layer. (This B-scan was produced using the "Hydra-Spectralis," which was developed by the HuCE-optoLab of the Bern University of Applied Sciences, Biel, Switzerland. For more technical information, see Appendix A.1.)

real value for choroidal thickness. However, in the datasets used in this study, these differences are marginal, and can be neglected.

\section{Intra-Retinal Layers}

The retina can be divided into eleven intra-retinal layers, consisting of seven inner-retinal (nos. (1)(7)) and four outer-retinal (nos. (8)-(11)) layers [91,92], as follows: (1) the inner limiting membrane (ILM), (2) the nerve fiber layer (NFL), (3) the ganglion cell layer (GCL), (4) the inner plexiform layer (IPL), (5) the inner nuclear layer (INL), (6) the outer plexiform layer (OPL), (7) the outer nuclear layer (ONL), (8) the inner/outer segment of the photoreceptors (IS/OS), (9) the external limiting membrane (ELM), (10) Bruch's membrane (BM), and (11) the retinal pigment epithelium (RPE). The aforementioned intra-retinal layers, as well as the choroid-sclera interface (CSI), are illustrated in Fig. 2.9.

According to the internationally reached consensus for OCT imaging terminology [93, 94], we must differentiate between the terms band, layer and zone. "Band" denotes 3D-volume structures in the retina. A "zone" is a region for which the contours are not clearly delineated, for example the choriocapillaris-Bruch's membrane-retinal pigment epithelium complex (CBR). While the GCL, the INL and ONL, the myoid zone (EZ), and the OS are hyporeflective because of their low light scattering potential, all others are capable of powerful light scattering and are hence 


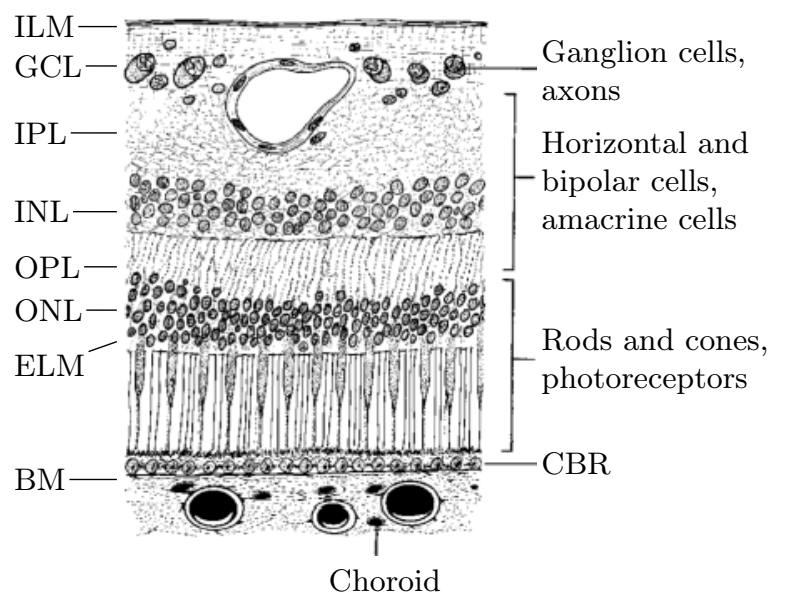

Figure 2.10.: Section of the central retina (source: [96]).

hyperreflective [95]. The outermost retinal layer is the hyperreflective CBR. The fovea is clearly visible because of its typical depression located in the middle of the OCT B-scan, see Fig. 2.9. This characteristic form is caused by the absence of inner layers at the macula ${ }^{8}$, leading to a thinning of the retina in this area.

The following points are worth mention regarding the thickness of intra-retinal layers $[62,97,98]$ :

- The central retina close to the fovea is considerably thicker than the peripheral retina, see Fig. 2.10. This is caused by the increased packing of photoreceptors, especially the cones, and their associated bipolar and ganglion cells, whereas the peripheral retina is mostly rod-dominated. The ONL, composed of the cell bodies of the rods and cones, shows a similar thickness in both parts of the retina. The OPL consists of neuronal synapses.

- The INL is thicker in the center of the retina than in its peripheral areas, due to a greater density of cone-shaped connecting second-order neurons (cone-shaped bipolar cells). It consists of cells that are closely packed, of which there are three varieties: bipolar cells, horizontal cells, and amacrine, see Fig. 2.10.

- A remarkable difference between the central and peripheral retina can be seen in the relative thickness of the IPL, GCL and NFL [97].

Although measuring intra-retinal layer thickness using OCT is not at the core of our work, it has received attention for use in preventing and monitoring several ocular diseases. For example, the thickness of the RNFL can help in diagnosing glaucoma [90,91].

\footnotetext{
${ }^{8}$ Actually, the radial shift of the neuronal and their support layers provides better visual acuity.
} 


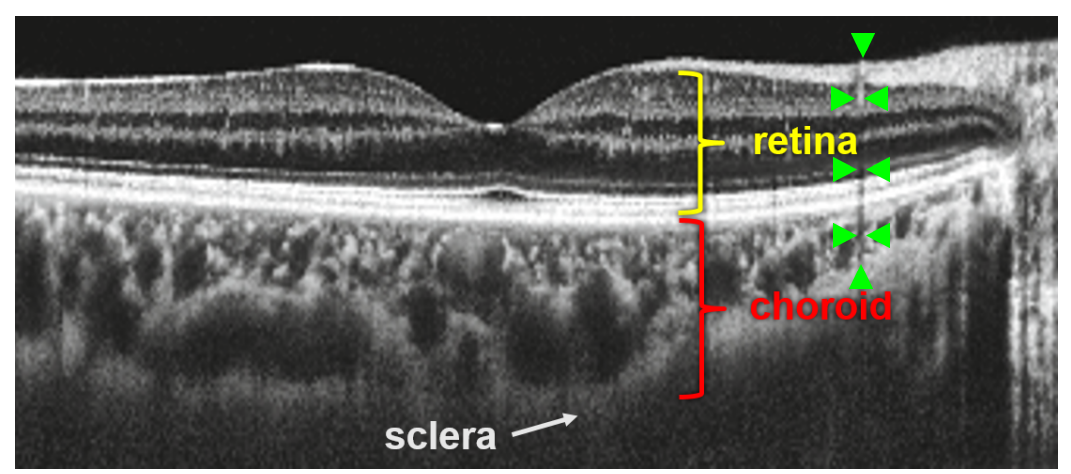

Figure 2.11.: Example of a less recognizable choroid-sclera interface.

In this OCT B-scan the choroid-sclera border region is not well visible, because of poor contrast, loss of signal and the presence of artifacts. The latter manifest themselves as a discontinuity of the image (see the area bordered by the green markers). (This image was kindly provided by the HuCE-optoLab of the Bern University of Applied Sciences, Biel, Switzerland.)

\subsection{Technical Background}

In this section we will first give an overview of the commonly used imaging techniques currently used in ophthalmology. Then we will go into the individual techniques with a special emphasis on OCT.

\section{General Remarks}

Fluorescein angiography (FA) [99-101] and optical coherence tomography (OCT) [102-104] are important medical imaging tools used in the examination and management of ocular diseases [100]. Often both imaging methods are necessary to arrive at a correct diagnosis and to facilitate treatment, so in that sense they complement each other. FA provides retinal circulation details, and OCT offers high-quality cross-sectional images of inner-ocular structures. Due to the injection of chemicals, FA is invasive and, as a non-amplified detection method, it is not ideal for patients with small pupils or insufficient media clarity [100]. In addition, the information provided by FA is qualitative, and interpretation of the results may be subjective [101].

OCT is objective, in other words less dependent on the investigators' skills, because this technique, due to its higher sensitivity, is not so much affected by pupil size or media clarity. Almost concomitantly with the advent of digital imaging, Wide Field Imaging (WFI) and Ultra Wide Field Imaging (UWFI), based on confocal scanning laser ophthalmoscopy (cSLO), have replaced traditional fundus cameras [105]. WFI allows to capture images beyond $50^{\circ}$ field area while UWFI systems allow for up to $200^{\circ}$. As a result, over $80 \%$ of the retinal surface area can be covered.

\section{Fluorescein Angiography}

FA is a medical imaging procedure which examines the blood circulation of the retina and choroid by increasing angiography contrast. Sodium fluorescein dye is injected into the bloodstream, and the blood vessels in the posterior part of the eye are illuminated by blue light at a wavelength of $490 \mathrm{~nm}$. As a result, the dye emits fluorescent green light, which is photographed, resulting in 


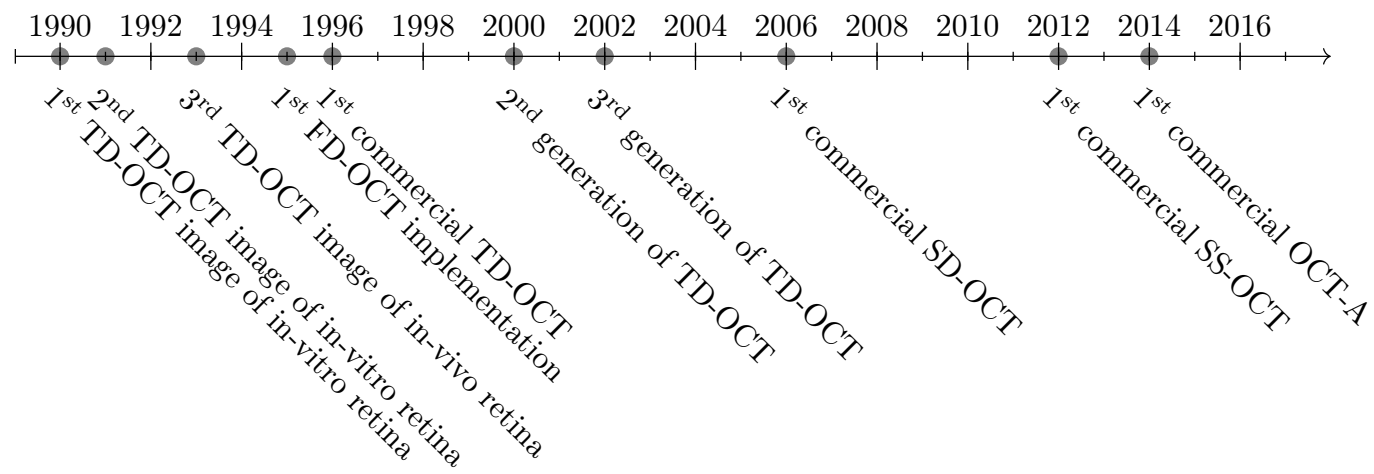

Figure 2.12.: Milestones in OCT.

Milestones in the development of OCT in ophthalmology on a timeline. (Figure adapted from [107].)

an angiogram [99-101]. Although the test does not involve any direct contact with the eyes, the use of sodium fluorescein dye is not completely risk-free [106].

Optical Coherence Angiography

OCT-A [100,108] has emerged in 2014 as a non-invasive technique for imaging the microvasculature of the retina and choroid by chemically increasing angiography contrast. In this procedure, repeated OCT scans acquired at the same position in the retina are utilized to detect minute changes. After correcting the effects of involuntary eye movements in the scans, the structures of the retinal tissue can be used as a static reference, in which only the differences due to blood flow in the vasculature remain as a result [95]. One of the main benefits is the fact that the use of exogenous dye is no longer necessary, thus making this procedure noninvasive. OCT-A has been demonstrated to be useful in monitoring several ocular diseases, such as diabetic retinopathy, retinal vein occlusion, and MacTel2 [109,110].

\subsubsection{General Information about Optical Coherence Tomography}

As aforementioned, OCT is a non-contact, non-invasive imaging technique, based on low coherence interferometry, to get high resolution retinal and choroidal images. This procedure is similar to ultrasound B-mode imaging except for the fact that it uses light instead of sound. Near-infrared light with a wavelength ranging from $750 \mathrm{~nm}$ to $1100 \mathrm{~nm}$ is used in vivo to generate tomographic images, because of its invisibility (so, the lack of brightness) and its depth penetration due to low scattering. These images reveal properties of biological microstructures, physiological information, blood flow, elasticity, changes in polarization states, and molecular content.

The basic principle of OCT is to acquire and measure the echo delay of the examined tissue's interior. This is achieved by detecting the interference signal between coherent light propagating in both the sample arm and the reference arm of a Michelson interferometer, see Fig. 2.14. Originally, OCT depth scans were made by scanning the reference arm of the Michelson interferometer, the so-called time domain (TD)-OCT. The first TD-OCT systems were presented in 1990 by Fercher [111] and 1991 by Huang [112]. In 1995, time domain OCT was succeeded by Frequency domain (FD)-OCT, a technology based on measuring the interference spectrum. 


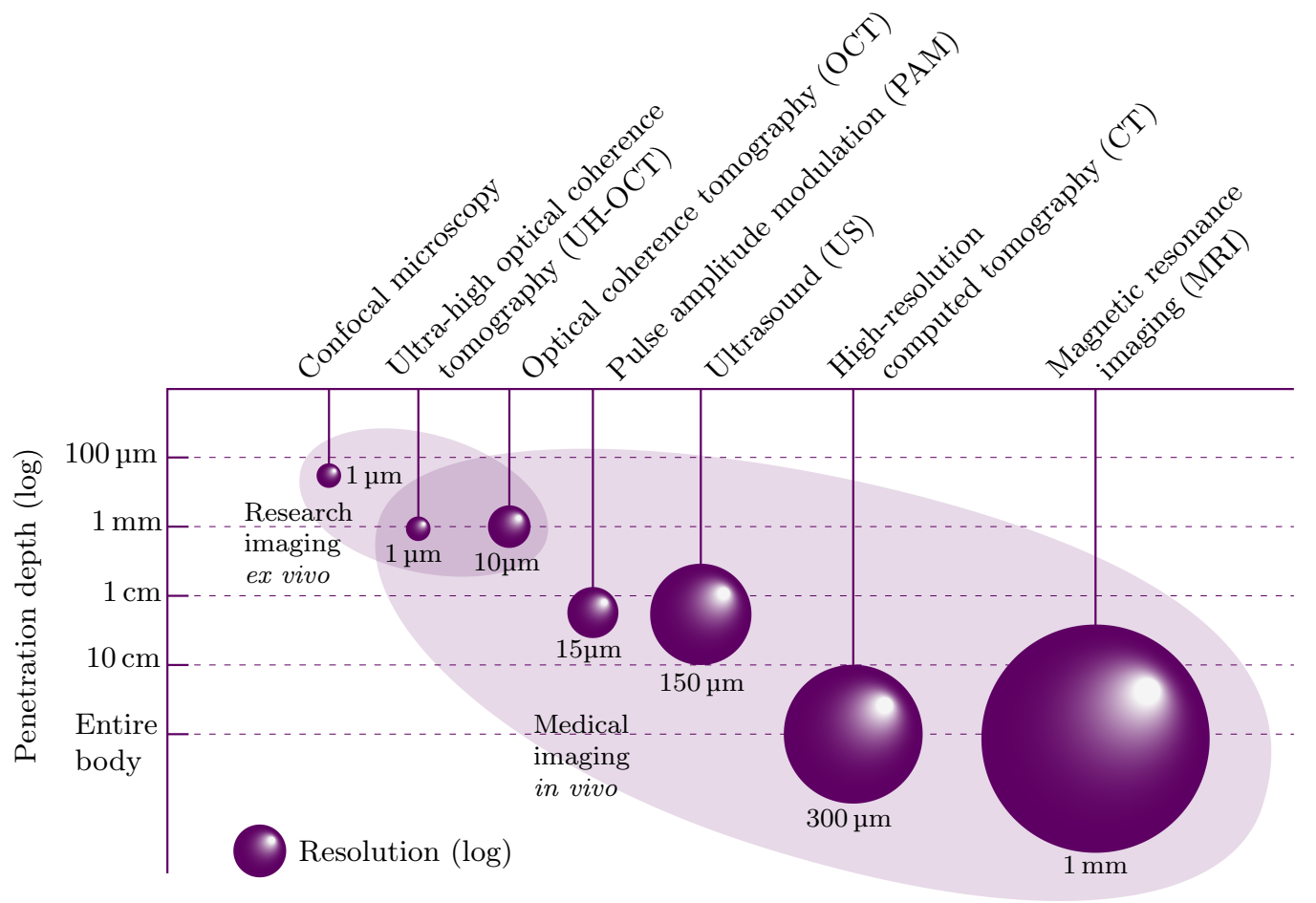

Figure 2.13.: Comparison of the different imaging modalities.

OCT compared in terms of resolution (the size of the "sphere") and penetration depth (the length of the "pendulum") with other clinical imaging technologies, such as optical (confocal microscopy), electric (PAM), acoustic (US), nuclear and X-ray (CT), magnet or radio-resonance (MRI). (Figure adapted from $[113,114]$.

(For a better understanding of the development of OCT in time, see Fig. 2.12.) With an axial resolution of $1-15 \mu \mathrm{m}$ and an imaging depth of about $2-3 \mathrm{~mm}$ in the tissue, OCT closes the gap between confocal microscopy and ultrasound, see Fig. 2.13. OCT allows a recording speed that is about 100 times faster than in normal ultrasound. Due to the high transparency of the human eye in this wavelength range, and the non-invasiveness of the procedure, OCT has proven to be a suitable method to characterize changes in the corneal, retinal and choroidal structures.

However, OCT has its limitations [115]: (1) Lateral resolution is limited by the numerical aperture $^{9}$, and therefore OCT requires a minimal pupillary diameter of $4 \mathrm{~mm}$ to obtain high quality images. (2) OCT has limited applications in the case of poor media clarity due to ocular diseases such as corneal edema or dense cataract. (3) High astigmatism, a decentered intraocular lens, or other alterations of optical surfaces, can compromise the quality of OCT scans. (4) Imaging related artifacts represent a problem. These anomalies in the scan do not represent the actual physical structure of the eye, see Fig. 2.11. Motion artifacts are the result of involuntary eye movements during scan acquisition such as patient blinks, microsaccades (small, fast, jerk-like eye movements), drifts (slow motions which appear during the intervals between

${ }^{9}$ The numerical aperture of an optical system is a (dimensionless) number that characterizes the range of angles over which the system can accept or emit light. For more details see Appendix C.1. 
(A)

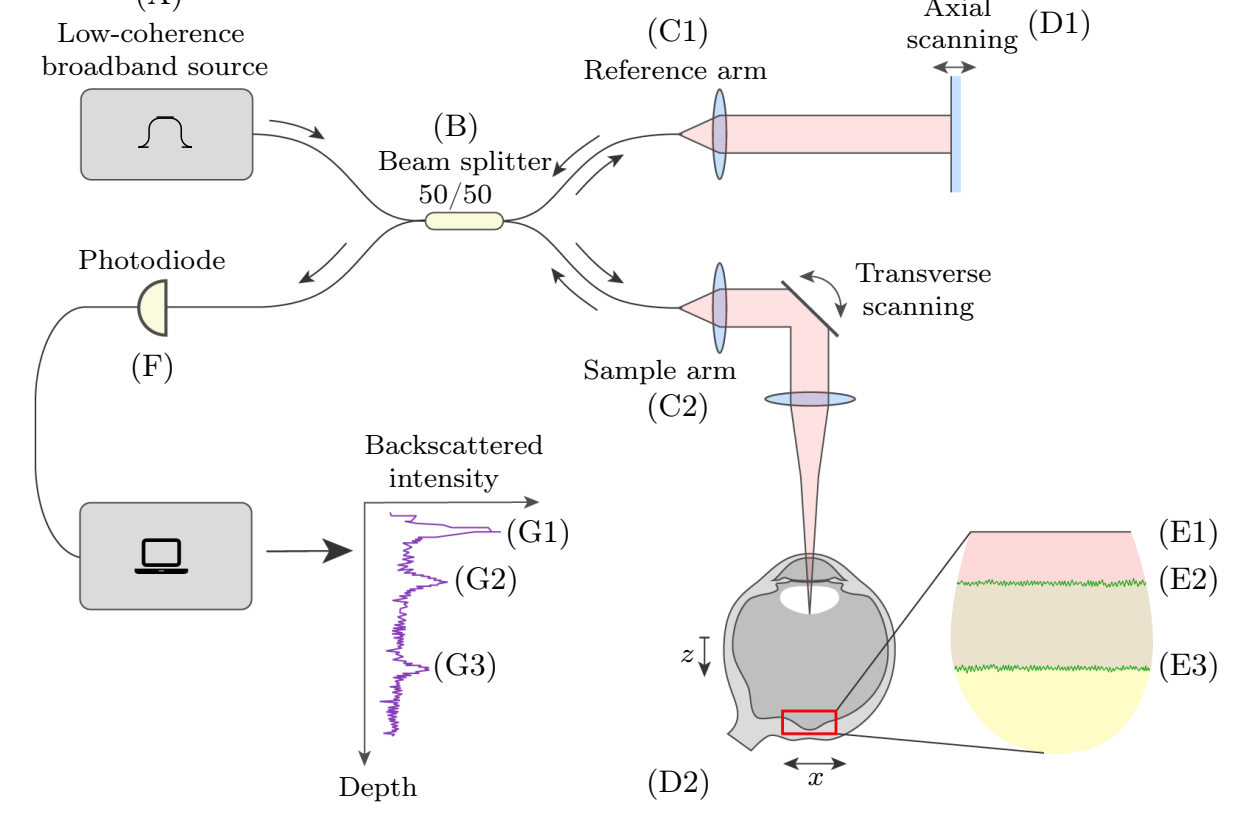

(C1)

Reference arm

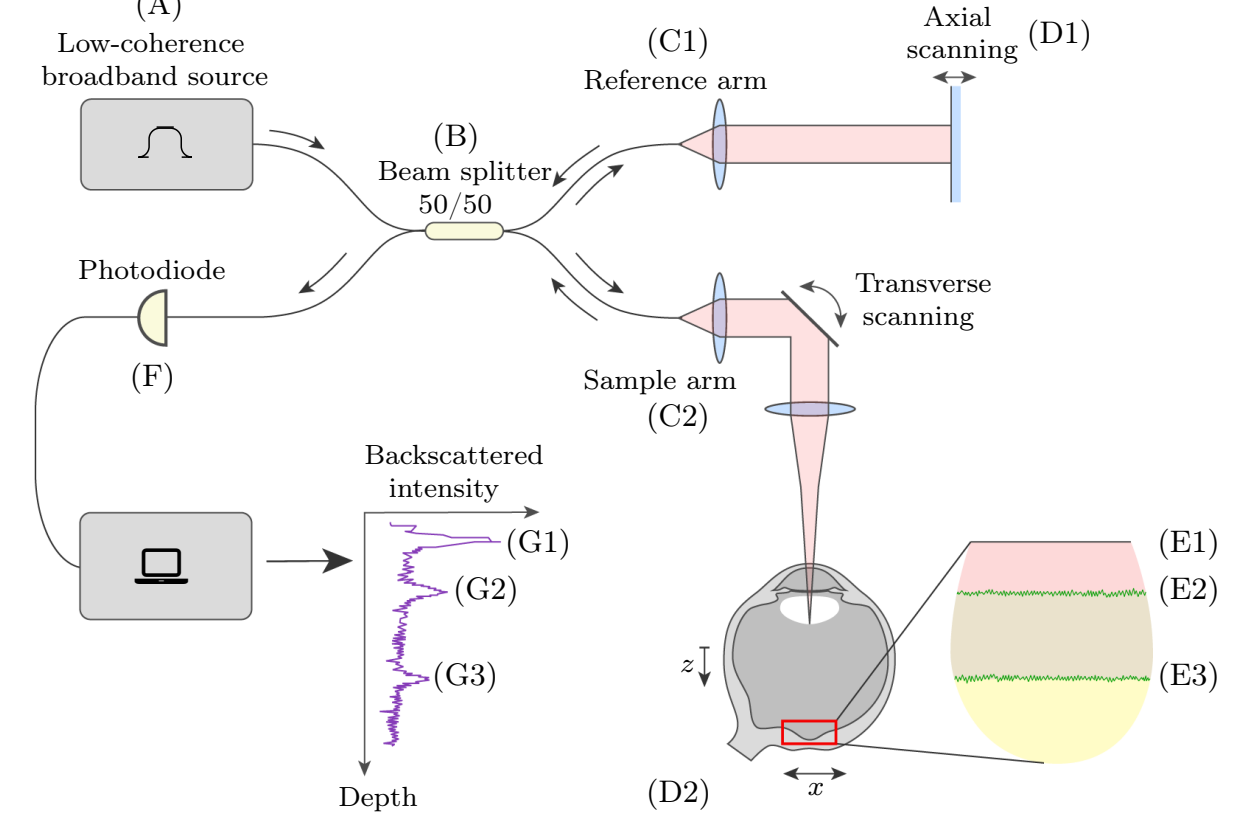

Axial (D1)

Figure 2.14.: Time domain OCT visualized.

Low time-coherence infrared light (A) goes through the beam splitter (B) into a reference arm $(\mathrm{C} 1)$ with a shifting mirror (D1), and a sample arm $(\mathrm{C} 2)$, leading to the posterior part of the eye (D2). The distance between beam splitter and reference mirror is continuously varied until the retinal/choroid tissue and the reference mirror have the same distance from the light source. Modulations in intensity, also called interference fringe bursts (E1-E3), are detected by the photodiode (F). As a result, an A-scan, a depth-resolved reflectivity profile (along the axial/ $z$-direction), is generated, where each peak location (G1-G3) corresponds to the depth of the tissue (E1-E3) at that position. (Figure adapted from [114].)

microsaccades). While microsaccades are responsible for breaks during the scan acquisition, drifts are the reason for shrunk and expanded areas in OCT data. Mirror artifacts are caused by the Fourier transformation used in FD-OCT devices, which cannot distinguish positive from negative time delays and, therefore, produce images that are symmetrical around the zero delay line $[116,117]$.

\subsubsection{Time and Frequency Domain OCT}

\section{Time Domain OCT}

Although TD-OCT has been widely superseded by FD-OCT, a basic knowledge of this technique is necessary to understand the principle of image acquisition used in this study. In TD-OCT $[118,119]$, a light beam emitted by a superluminescent diode (a low-coherence light source) is divided by a beam splitter in such a way that half of the beam is sent into the eye and backscattered by the ocular media. The other half is reflected by a reference mirror at similar optical distance from the beam splitter. Both beams are then mixed by the beam splitter 


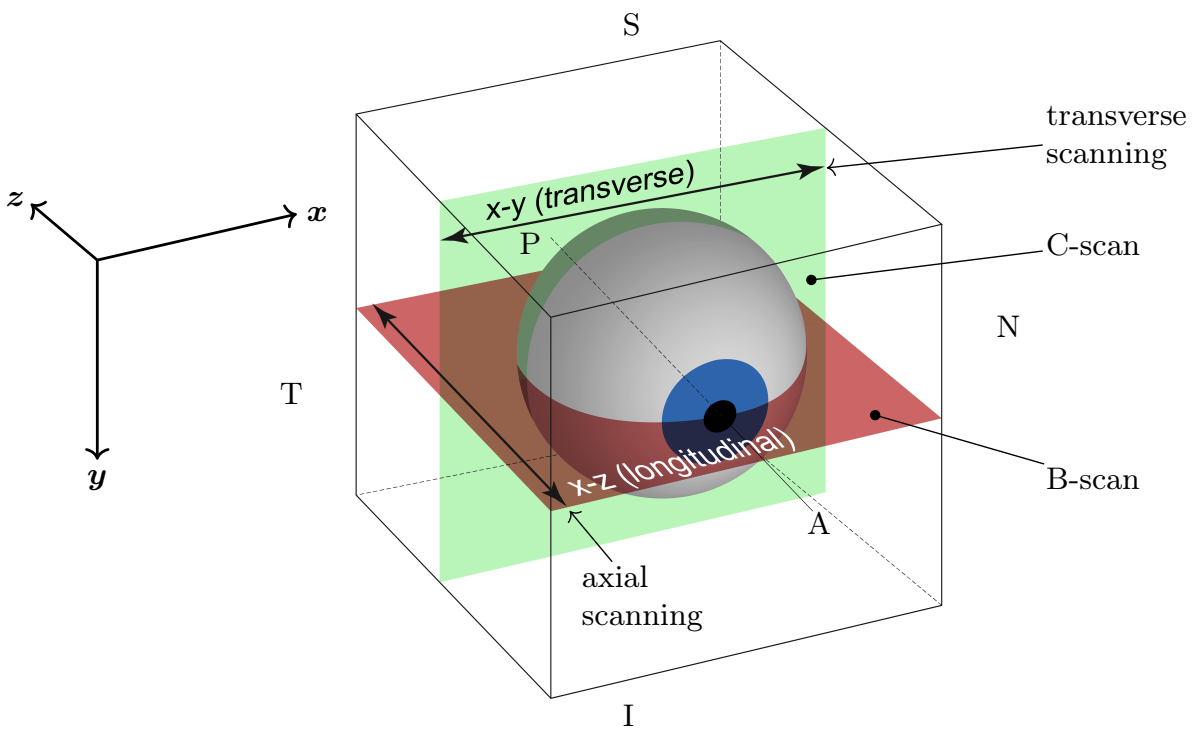

Figure 2.15.: The OCT scanning terminology with relative anatomical orientation.

An A-scan is produced along the $z$ /axial-direction. When the $z$-is combined with the $x$ /lateral-direction a $2 \mathrm{D}$ cross-section, or longitudinal slice, called B-scan, is the result. Adding the $y /$ transverse-direction generates a $3 \mathrm{D}$-volume stack of parallel Bscans, called a C-scan. Translated into standard medical terms to define anatomical positions, the directions and cross-sections are labeled: "Temporal" (T), "Nasal" $(\mathrm{N})$, "Posterior" (P), "Anterior" (A), "Superior" (S), and "Inferior" (I).

and directed to a detector. If the optical path lengths match within a coherence length, and therefore the time traveled by the light is nearly equal in both arms, the interference happens for all wavelengths at once, and this signal change is recorded. By modifying the position of the reference mirror, various retinal structures at different depths are detected, see Fig. 2.14.

The obtained 1D tissue-reflectance information, or reflectivity profile in depth $(z$-/axial direction), is called $A$-scan, analogous to the terminology used in ultrasound, namely amplitude mode scan. By transverse scanning (in $x$-/lateral direction) of the retina, a 2D cross-sectional image, called B-scan, is generated by collecting successive A-scans. This abbreviation also originated in ultrasound imaging, where B-scan means brightness mode scan. For a better understanding of the scanning terminology see Fig. 2.15.

\section{Frequency Domain OCT}

The second generation of widely implemented ophthalmic OCT technology, the so-called Frequency domain (FD)-OCT [120], implements the principle of low-coherence interferometry more efficiently by utilizing the Fourier transform relations between time and frequency. FD-OCT became extremely successful because the associated technologies became cheaper and available with the advent of cheap line sensor cameras (of which the sensor focuses on only one line at the time). Without the need of mechanical scanning of the optical path length, FD-OCT, unlike TD-OCT, uses spectral information to generate A-scans. Instead of moving the reference arm for each A-scan and measuring the resulting intensities with a single photodetector, the reference mirror is kept stationary and the interference signal is analyzed by a spectrometer. 


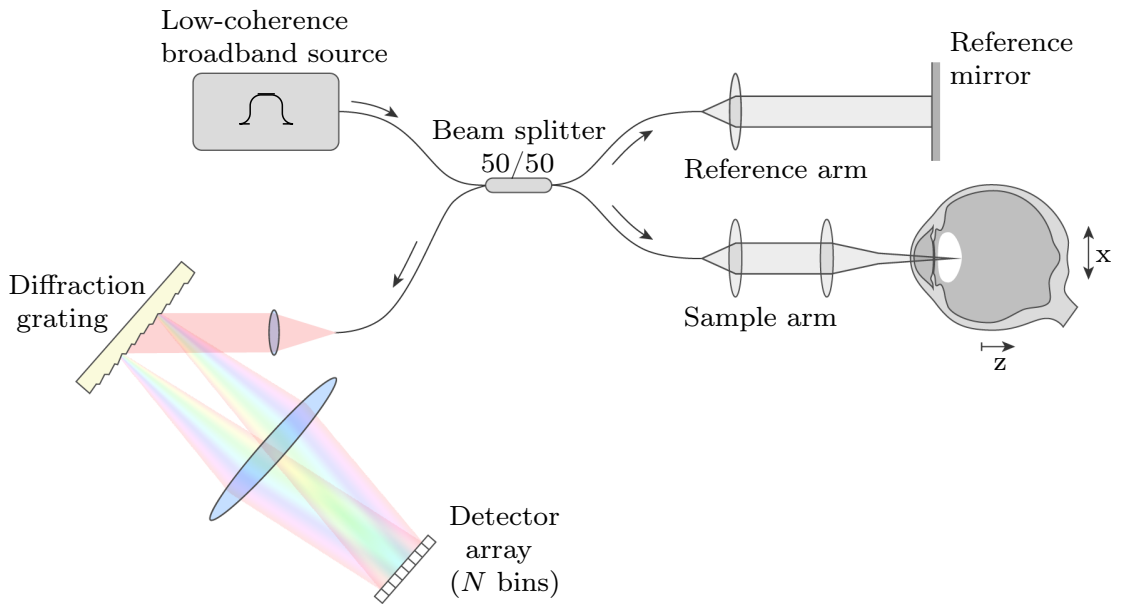

(a) Spectral domain (SD)-OCT system

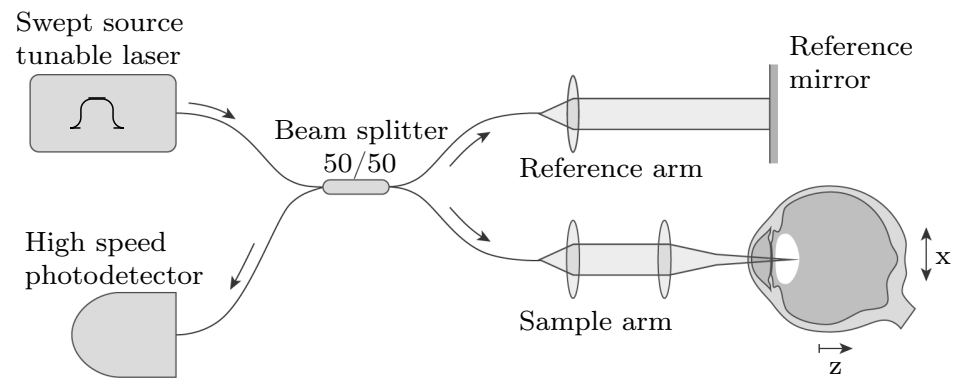

(b) Swept Source (SS)-OCT system.

Figure 2.16.: The two types of Frequency domain OCT systems.

(a) While SD-OCT uses a spectrometer for wavelength separation, (b) SS-OCT features a light source which sweeps the wavelength in time. Both technologies record an interference spectrum which carries the depth information of the sample and uses the inverse Fourier transform to convert the interference signal in an Ascan. (Figure adapted from [114].) 
Two methods, Spectral Domain (SD)-OCT and Swept Source (SS)-OCT, prevail when acquiring spectral information of the interferometric signal. ${ }^{10}$ While in SS-OCT the signal is time encoded, i.e. recorded sequentially, in SD-OCT (often also called "Fourier domain OCT"11) the signal is spatially encoded, i.e. spatially split, but recorded simultaneously. Both techniques generate an A-scan using the Fourier transform on the basis of the recorded interference spectrum, also called spectral interferogram (for mathematical details, see below).

In SD-OCT most of the components are identical to the setup of TD-OCT, but with the main difference that the point detector is replaced by a spectrometer, see Fig. 2.16(a). This allows capturing depth data of one A-scan simultaneously and not sequentially as in TD-OCT. In order to spatially separate the different wavelength contributions into a line image, which is recorded by a high speed line-scan camera, the spectrometer uses a diffraction grating to produce an interferogram of the spectral fringe patterns. As a broadband light source, a superluminescent diode is commonly chosen, due to its large bandwidth, reasonable cost, and high brilliance (the power density in a single lateral mode of a fiber).

The principle of SS-OCT [122] succeeded SD-OCT and was applied to measure intraocular distances [123]. Its optical setup is similar to TD-OCT, but it uses a wavelength-tunable laser which rapidly sweeps a narrow line-width over a broad range of wavelengths and the interferometric signal is detected sequentially by a single high speed photo-detector, at even better SNR (signal to noise ratio), see Fig. 2.16(b).

\section{Frequency vs Time Domain OCT}

FD-OCT has several advantages compared to TD-OCT: higher patient comfort, an increased acquisition speed and a significantly improved signal-to-noise ratio. It also reduces motion artifacts and shows a higher sensitivity, which makes FD-OCT a powerful tool for acquiring in-vivo tomograms of the human retina and choroid. Within FD-OCT, SS-OCT offers more advantages than SD-OCT in terms of increased imaging depth, higher detection performance, improved imaging range and an adaptability to different, especially longer wavelengths. For an overview of the different techniques, see Table 2.1. In contrast to TD-OCT, which is not yet suitable for 3D imaging due to the limited acquisition speed of A-scans (and, therefore, almost only enables "in vivo" $2 \mathrm{D}$ imaging), FD-OCT even allows $3 \mathrm{D}$ reconstruction of the internal structure of the eye in the form of a 3D volume-scan, also called $C$-scan, or en face image. C-scans in FD-OCT are compilations of the data of all B-scans, enabling a global overview of the morphology of the retina or choroid. For a better understanding of the different scan-types, see Fig. 2.17.

\section{Enhanced Depth Imaging}

In FD-OCT the detection range is related to the spectral resolution with which the signal can be detected, and is characterized by a depth-depending signal "roll-off" effect. This so-called "roll-off sensitivity" defines the reduction in sensitivity which FD-OCT inherently exhibits with increasing axial distance from the zero delay point (where sample and reference feature the same length). In contrast to TD-OCT, a modification to the standard technique in conventional commercial FD-OCT imaging systems allows to image the choroid by shifting the zero delay

\footnotetext{
${ }^{10}$ The terms "spectral domain/spectrometer-based OCT" should not be confused with "spectroscopic OCT", an extension of OCT technology in which not only the structural information of the ocular tissues is gathered, but also spectroscopic tissue parameters, such as blood absorption, and oxygen saturation are examined. [121]).

${ }^{11}$ The term "Fourier domain OCT" is often used instead of the more appropriate term "Frequency domain OCT". In signal processing there is a "time domain" and a "frequency domain", but no "Fourier domain".
} 


\section{Background}

\begin{tabular}{|c|c|c|c|c|c|c|c|c|}
\hline & $\begin{array}{c}\text { Type of } \\
\text { OCT }\end{array}$ & $\begin{array}{l}\text { Echo time delay } \\
\text { measurement }\end{array}$ & $2 \mathrm{D} / 3 \mathrm{D}$ & $\begin{array}{l}\text { Light source, } \\
\text { detector, and } \\
\text { mirror }\end{array}$ & $\begin{array}{l}\text { Scanning } \\
\text { speed }\end{array}$ & $\begin{array}{c}\text { Axial } \\
\text { resolution }\end{array}$ & $\begin{array}{l}\text { Transverse } \\
\text { resolution }\end{array}$ & Range of imaging \\
\hline & $\begin{array}{l}\text { Time } \\
\text { Domain } \\
\text { (TD) }\end{array}$ & $\begin{array}{l}\text { Sequential (by a } \\
\text { depth scanner) }\end{array}$ & $2 \mathrm{D}$ & $\begin{array}{l}\text { Superluminescent } \\
\text { diode }(810 \mathrm{~nm}), \\
\text { single photon } \\
\text { detector, moving } \\
\text { mirror }\end{array}$ & $\begin{array}{c}400 \\
\text { A-scans/sec }\end{array}$ & $10 \mu \mathrm{m}$ & $20 \mu \mathrm{m}$ & $\begin{array}{c}\text { Vitreoretinal } \\
\text { interface to RPE (no } \\
\text { EDI possible) }\end{array}$ \\
\hline \multirow{2}{*}{$\begin{array}{l}\text { Frequency } \\
\text { Domain } \\
\text { (FD) }\end{array}$} & $\begin{array}{l}\text { Spectral } \\
\text { Domain } \\
\text { (SD) }\end{array}$ & $\begin{array}{l}\text { Simultaneous (by } \\
\text { a spectrometer) }\end{array}$ & $3 \mathrm{D}$ & $\begin{array}{c}\text { Broadband } \\
\text { superluminescent } \\
\text { diode source } \\
(840 \mathrm{~nm}), \text { array of } \\
\text { detectors, fixed } \\
\text { mirror }\end{array}$ & $\begin{array}{c}27^{\prime} 000- \\
70^{\prime} 000 \\
\text { A-scans } / \text { sec }\end{array}$ & $5-7 \mu \mathrm{m}$ & $14-20 \mu \mathrm{m}$ & $\begin{array}{l}\text { Posterior cortical } \\
\text { vitreous to sclera } \\
\text { (using EDI mode) }\end{array}$ \\
\hline & $\begin{array}{l}\text { Swept } \\
\text { Source } \\
\text { (SS) }\end{array}$ & $\begin{array}{l}\text { Sequential (by a } \\
\text { high speed } \\
\text { photo-detector) }\end{array}$ & $3 \mathrm{D}$ & $\begin{array}{c}\text { Swept Source } \\
\text { tunable laser } \\
1500 \mathrm{~nm} \text { single } \\
\text { detector }\end{array}$ & $\begin{array}{c}100^{\prime} 000- \\
400 ' 000 \\
\text { A-scans } / \text { sec }\end{array}$ & $5 \mu \mathrm{m}$ & $20 \mu \mathrm{m}$ & $\begin{array}{c}\text { Posterior cortical } \\
\text { vitreous to sclera } \\
\text { (superior to SD-OCT } \\
\text { using EDI) }\end{array}$ \\
\hline
\end{tabular}

Table 2.1.: The different types of OCT compared. (Table adapted from [107].)

line or point of highest sensitivity from its standard position, more toward the sclera, so that the roll-off compensates some of the depth-dependent scattering losses. This so-called Enhanced Depth Imaging [124,125], as of now EDI, enables an overall assessment of the choroid and the choroid-sclera interface. The SNR in deep choroidal images is improved by shifting the image origin as well as by averaging multiple (50-100) B-scans.

Despite the aforementioned advantages, EDI is limited by the appearance of mirror artifacts inside the choroid, which are intrinsic to the FD-OCT signal, see Fig. 2.18. Another limitation is a lack of automated measurement software, which, due to the manual alternative, could easily lead to bias. Besides, signal transmission and reception between the OCT and the choroid is hindered as a result of media opacities. Although appropriate for analyzing the choroid in its full thickness, EDI does not contribute significantly to investigate the choriocapillaris and choroidal microvasculature, due to their proximity to the RPE [124].

\section{Challenges in Choroidal Imaging}

Although they are very good at providing retinal detail, even commercial SD-OCT $870 \mathrm{~nm}$ wavelength devices cannot completely reproduce choroidal details because: (1) the RPE and the choroid do not allow sufficient signal penetration [126], due to the depth-depending roll-off sensitivity affecting the detected signal, as well as the various inherited limitations with Fourier transformation [127], (2) at the level of the choroid the light beam is scattered by the tissue, (3) there is not enough contrast between structures of critical interest, and (4) the scan does not have an ideal lateral resolution [127].

Visualizing the full thickness of the choroid is challenging caused by loss of signal due to the scattering and absorption of the incident light by the pigment cells in the RPE. As mentioned before, $870 \mathrm{~nm}$-wavelength OCT is not enough for tissue imaging at the posterior part of the eye, such as the choroid and sclera. In order to penetrate into the choroid, the use of a light source with longer wavelength is necessary [126]. Unfortunately, this is not possible with most commercial OCT devices without affecting the quality of retinal images. In this context, the main body of the images used in this study was acquired with an improved SD-OCT based Spectralis device, which was extended with a second detection arm operating at a depth of $1075 \mathrm{~nm}$ wavelength, favoring deeper penetration into choroidal tissue. This device, called "Hydra-Spectralis", was developed by the HuCE-optoLab of the BUAS. For more technical information, see Appendix A.1. 

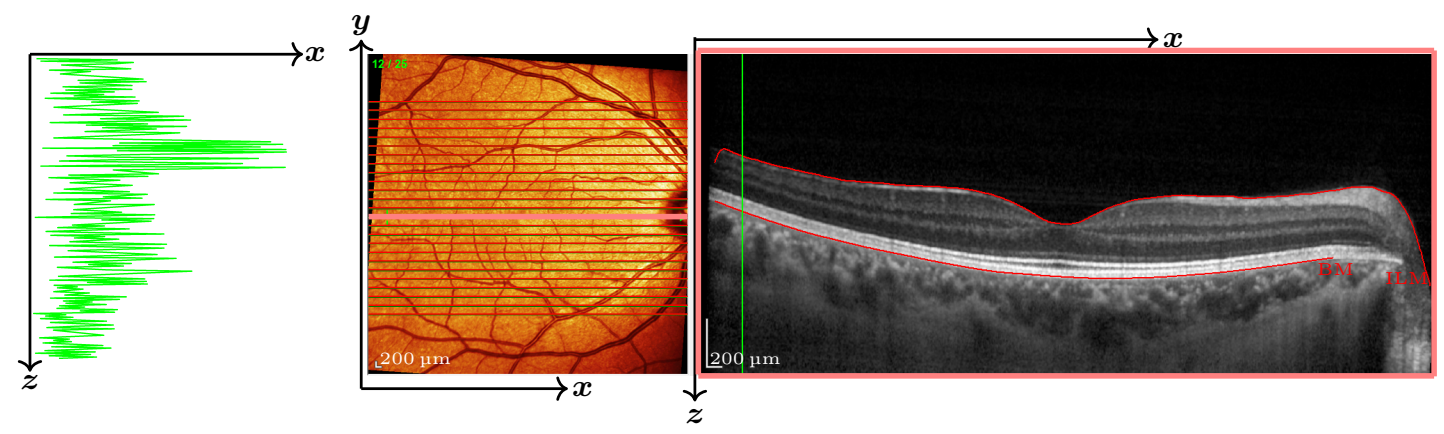

(a) A-scan

(b) B-scan

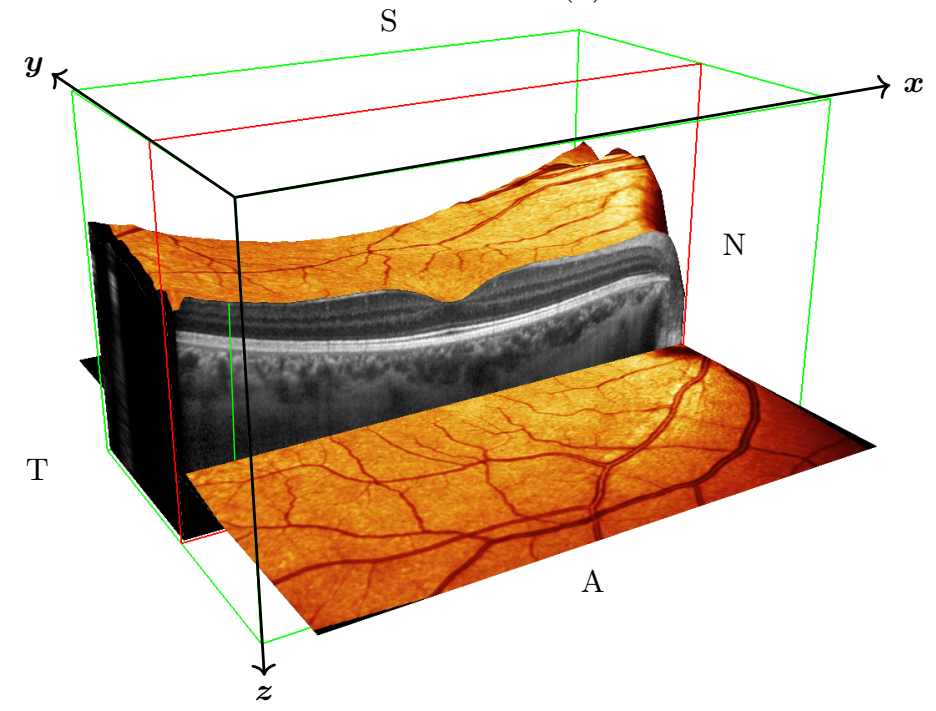

(c) C-scan

Figure 2.17.: OCT scan-types.

(a) The A-scan is a 1D scan along the $z$-axis, containing depth information. (b) The B-scan shows a 2D cross-section in which one axis is the depth axis. (The green vertical line in the B-scan, as well as the one in the $\mathrm{C}$-scan, indicates a single Ascan. The thicker horizontal line in the middle of the fundus image on the left side in (b) corresponds to the B-scan depicted on the right.) (c) The 3D-volume C-scan combines the information of all A- (green vertical line) and B-scans (surrounded by the red rectangle). (Images (b) and (c) were kindly provided by the HuCE-optoLab of the BUAS.) 


\section{Background}

\subsubsection{Mathematical Background of FD-OCT}

To fully appreciate the working principle of FD-OCT and to understand the formation of the spectral interferogram, a closer look at the signal formation is taken in the following section. For the sake of simplicity, the one-dimensional approximation is assumed, which reduces the typically utilized Gaussian beam to a mere plane wave.

In wave mechanics [128] a one-dimensional wavefunction takes the general form

$$
\psi_{n}(t)=\hat{\psi}_{n} \cdot \cos \left(k_{n} z_{n}-\omega_{n} t+\phi_{n}\right)=\Re\left[\Psi_{n}(t)\right],
$$

where $\hat{\psi}_{n}>0$ is the electromagnetic wave's amplitude, $\phi_{n}$ the phase angle, $k_{n}$ the wavenumber, and $\omega_{n}$ the angular frequency. In other words, it can be represented as the real part $\Re\left[\Psi_{n}(t)\right]$ of a complex wavefunction $\Psi$ of the form

$$
\Psi_{n}(t)=\hat{\psi}_{n} \cdot \mathrm{e}^{j\left(k_{n} z_{n}-\omega_{n} t+\phi_{n}\right)}=\hat{\psi}_{n} \cdot\left(\cos \left(k_{n} z_{n}-\omega_{n} t+\phi_{n}\right)+j \sin \left(k_{n} z_{n}-\omega_{n} t+\phi_{n}\right)\right) .
$$

Nevertheless, the complex form

$$
\Psi_{n}(z, t)=\hat{\Psi}_{n} \cdot \mathrm{e}^{j\left(k_{n} z_{n}-\omega_{n} t\right)}, \text { where } \hat{\Psi}_{n}=\mathrm{e}^{j \phi_{n}}
$$

is often preferred to the more straightforward real one, as it allows to combine the amplitude $\hat{\psi}_{n}$ and the phase angle $\phi_{n}$ into a single complex amplitude $\hat{\Psi}_{n}$.

As illustrated in Fig. 2.14 and 2.16 the idea behind an OCT system is to measure the optical delay of back reflected electromagnetic waves within a spectral bandwidth to reveal the depth in which the reflection occurred. Since the delays of the back-reflected waves are not measurable in a direct way, a reference is needed. With the help of an optical beam splitter, half of the incident light is sent to the sample while the other half is directed to a reference arm with a known length, see [129]. Since both beams stem from the same source, they will initially have the same wavenumber $k$ and phase $\phi$, but at the interference point their amplitudes can differ. Please note that the time dependency $\mathrm{e}^{j \omega t}$ of the optical field is neglected, as only the oscillation period is relevant for the signal detection.

Without loss of generality, we consider the case of a single reflector in the sample arm (i.e. $n=1$ ) and denote the power reflectivity, i.e. the ability to reflect or backscatter light, of the reference mirror and the sample layer by $R_{r}=\left|\hat{\Psi}_{r}\right|^{2}$ and $R_{s}=\left|\hat{\Psi}_{s}\right|^{2}$, respectively [130]. This leads to the following simplified superposition

$$
\Psi_{\text {sum }}=\Psi_{r}(k)+\Psi_{s}(k)=\hat{\Psi}_{r} \cdot \mathrm{e}^{j\left(k z_{r}\right)}+\hat{\Psi}_{s} \cdot \mathrm{e}^{j\left(k z_{s}\right)} .
$$

The light field's intensity $I(k)$ (here defined as the magnitude of the Poynting vector) as measured by the interferometer is described by the product of a plane electromagnetic wave with its conjugate according to

$$
P \propto I(k)=\left|\Psi_{\text {sum }}\right|^{2}=\Psi_{\text {sum }} \cdot \bar{\Psi}_{\text {sum }}
$$

In other words, the optical power $P$ is proportional to the square of the absolute value of $\Psi$ (or 
the product of $\Psi$ with its complex conjugate). According to Eq. (2.6) and Eq. (2.5) we obtain

$$
P \propto I(k)=\left|\Psi_{r}+\Psi_{s}\right|^{2}=\left(\Psi_{r}+\Psi_{s}\right) \cdot\left(\overline{\Psi_{r}+\Psi_{s}}\right)=\ldots=\left|\hat{\Psi}_{r}\right|^{2}+\left|\hat{\Psi}_{s}\right|^{2}+2 \hat{\Psi}_{r} \hat{\Psi}_{s} \cos (2 k \Delta z)
$$

where $\Delta z=z_{s}-z_{r}$ represents the optical path length difference between the signal and reference arms of the interferometer. The interferences at the output are due to the optical path difference $\Delta z$, which is defined as half of the optical path length difference between the reference mirror and the sample layer.

Therefore, in the case of a single reflector in the sample arm, the interference signal $I(k)$ (detected in the frequency domain) is given by

$$
I(k)=S(k) \cdot\left(R_{r}+R_{s}+2 \sqrt{R_{r} R_{s}} \cos (2 k \Delta z)\right),
$$

where $S(k)$ denotes the optical power density of the light source, described as a function of the wavenumber $k=\frac{2 \pi}{\lambda}$. According to the convolution theorem, the Fourier transform of a convolution " $\otimes$ " of two signals in the spatial domain is the pointwise product "." of their Fourier transforms in the frequency domain. By applying the inverse Fourier transform to Eq. (2.7) it follows:

$$
I(z)=\mathcal{F}^{-1}\{S(k)\} \otimes \mathcal{F}^{-1}\left\{\left(R_{r}+R_{s}\right)+2 \sqrt{R_{r} R_{s}} \cos (2 k \Delta z)\right\},
$$

where " $\otimes$ " denotes the convolution of both inverse Fourier transformed functions. Considering the relationship $\mathcal{F}^{-1}[\cos (2 k \Delta z)]=\delta(z-\Delta z)+\delta(z+\Delta z)$, we obtain

$$
I(z)=\mathcal{F}^{-1}[S(k)] \otimes\{\underbrace{\left(R_{r}+R_{s}\right) \delta(z)}_{(1) \text { DC }- \text { term }}+\underbrace{2 \sqrt{R_{r} R_{s}}[\delta(z-\Delta z)+\delta(z+\Delta z)]}_{(2) \text { complex conjugate artifact }}\} .
$$

Term (1) in Eq. (2.9), also called DC-term, is due to the non-interfering components of the sample and reference arms and indicates a peak at zero path length mismatch. Term (2) reveals the location of the scatterer and its indistinguishable complex conjugate image, which appears on the opposite side of zero path length (i.e. at the reference reflector position) ${ }^{12}$, see Fig. 2.18 (The slow electronic detector does not measure the phase of the interferogram, and, thus, the location of the scatterer can only be determined as an absolute, sign-less value [102].) This signal mirroring, referred to as the complex conjugate artifact, reduces the usable depth range in standard FD-OCT by half, see Fig. 2.18.

From a mathematical point of view, $I(z)$ is not directly the reflector position, but represents an entire $A$-scan. Looking for peaks/maxima in this signal gives the position of the reflector (in the spatial domain). In the more general case, a sample of $n \in \mathbb{N}$ reflective layers, each of them characterized by its corresponding depth position $z_{s_{i}}$ and its power reflectivity $R_{s_{i}}$, leads to a superposition of many different modulation patterns. Furthermore, let $\Delta z_{s_{i}}=z_{s_{i}}-z_{r}$ be defined as half of the optical path difference between the reference mirror and the $i^{\text {th }}$ layer (or

\footnotetext{
${ }^{12}$ From a mathematical point of view, the complex conjugate artifact in FD-OCT is a consequence of the fact that the detected interferometric spectrum is necessarily real, and, therefore, its inverse Fourier transform must be Hermitian symmetric [103].
} 

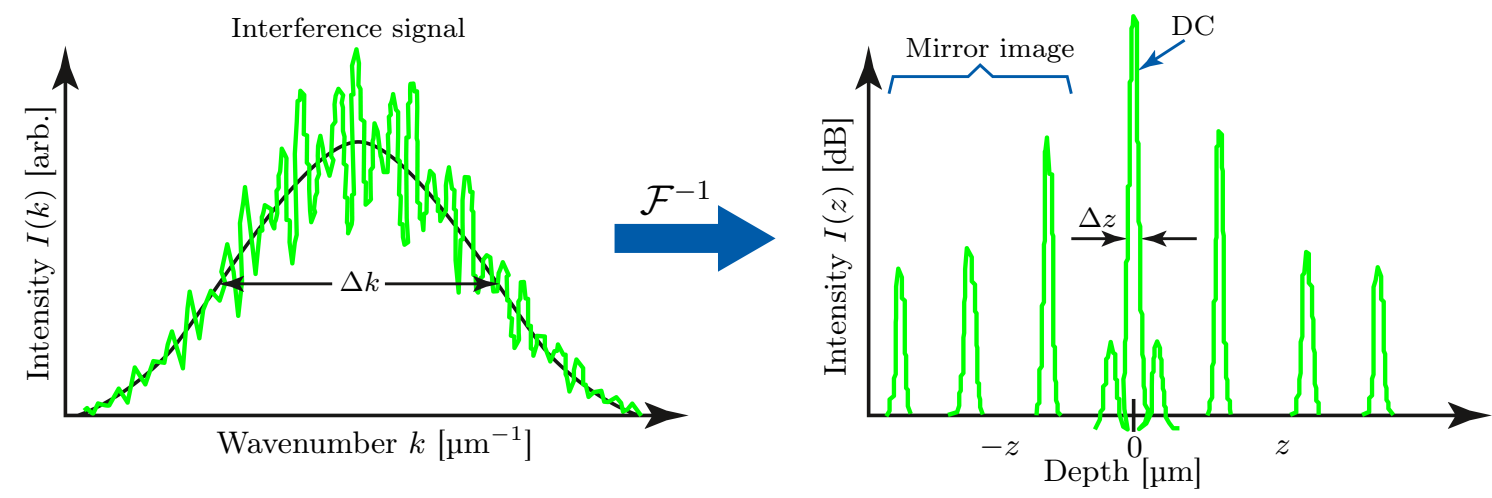

Figure 2.18.: Signal mirroring in FD-OCT.

FD-OCT records an interference spectrum that indicates the depth of the sample. Using the inverse Fourier transform, the interference signal is transformed into a depth-resolved reflectivity profile (A-scan) of the sample. The picture on the right side illustrates the mirror image generated during FD-OCT (as the result of the inverse Fourier transform of the spectral domain signal). For more details about axial and lateral imaging parameters in OCT see Appendix C.1. Note, that "arb." is the abbreviation for "arbitrary (unit)". (Figure adapted from [131].)

sample reflector), the interferogram $I(k)$ acquired by an $n$-array spectrometer is given by

$$
\begin{aligned}
I(k)=S(k)\left(R_{r}+\sum_{i=1}^{n} R_{s_{i}}\right. & +2 \sqrt{R_{r}} \sum_{i=1}^{n} \sqrt{R_{s_{i}}} \cos \left(2 k \Delta z_{s_{i}}\right) \\
& \left.+2 \sum_{i=1}^{n} \sum_{\substack{j=1 \\
j \neq i}}^{n-1} \sqrt{R_{s_{i}} R_{s_{j}}} \cos \left(2 k \Delta z_{s_{j}}\right)\right),
\end{aligned}
$$

where $\Delta z_{s_{j}}=z_{s_{j}}-z_{s_{i}}$ denotes the optical path difference between the two different sample reflectors. The location of the reflector is revealed by taking the inverse Fourier transform of Eq. (2.10). (For a comprehensive and detailed explanation the reader is referred to [103,104,130-132].) 


\section{Mathematical Principles underlying the Regularized Piecewise Rigid Registration Approach CRAR}

In this chapter, the mathematical principles and machine learning tools which play an essential role in this thesis are presented. For the underlying mathematical definitions and proofs see Appendix B.1 and B.2.

\subsection{Image Registration}

\subsubsection{General Remarks}

Different images of the same objects, taken at different points in time and on the basis of different angles or sensors, lead to a variations of the background, texture size and noise level which usually are no problems for the human visual system to interpret, but represent a challenging problem for a computer algorithm. Image registration [133-136] tries to overcome this problem and has as a fundamental goal to automatically estimate the correspondence between the images by finding the underlying spatial transform mapping that links them. More concretely, a pointto-point correspondence between the images is sought or, if necessary, assumed, so that the information within an image ("template") can be transferred to its original ("reference"), see Fig. 3.1. The main application domains of image registration include medical imaging [137, 138], remote sensing [139,140] and computer vision [141]. Historically, where images need to be rotated and shifted with respect to each other to achieve correspondence (e.g. in astronomy to register satellite images), image registration has been rigid, due to the inherent reproducible imaging geometry and static nature of the object. In medical image analysis, non-rigid image registration [142] is required. This is caused by the fact that an imaged body part (as in our case the choroid) undergoes a soft-tissue type of deformation [137,143].

\section{Intensity- and Feature-based Image Registration}

Independent of the difference rigid or non-rigid, there are two fundamental approaches to image registration: intensity-based and feature-based. Intensity-based registration methods define the similarity measure directly on image intensities $[134,137,138]$ and do not require any feature extraction or matching steps. They use dense voxel-wise similarity measures, which are suitable to estimate dense deformation fields. Intensity based similarity measures are distinguished by their application area into monomodal or multimodal.

Feature-based image registration, on the other hand, requires pre-processing steps to extract geometrical features (corner points or edges) in order to be able to match these with their 
counterparts in the second image. The main advantage of these methods is their dimensionality reduction property, which significantly reduces the computational time and load.

However, most recent research in image registration is intensity-based, because the registration procedure uses all the image information available.

\section{Relevance of Image Registration in OCT Imaging}

Image registration is vital for OCT image processing. This procedure is used to compare OCT B-scans within one stack (intra-stack) and between various stacks (inter-stack) in both crosssectional and longitudinal studies. In the latter, registration can also be applied on images taken at different points in time for monitoring long-term progression of diseases or the follow-up of the outcome of a treatment $[134,138]$. With the help of this kind of procedure, it is possible to correct inadvertent eye movements, tilting, bad illumination and noise.

\section{Four Components of Image Registration}

An image registration algorithm consists of four main components: the similarity measure, the transformation model, the optimization method and the regularization term. The similarity measure is usually an objective function that shows a relationship between two images, and has to be optimized. The transformation model defines the set of admissible transformations which, ideally, model the real transformations that the imaged objects, in order to be aligned, may physically undergo.

The optimization method is an algorithm that tries to find the best possible solution for a registration problem. In case of a parametric model, optimization is commonly performed using Gradient Descent in order to find the set of parameters which minimizes an energy functional, or loss function, as explained later in Sec. 3.2. ${ }^{1}$ In the non-parametric case, optimization can be done by solving partial differential equations $[149,150]$. (Recently, new methods have been developed, in which this tedious computational procedure is no longer necessary [151].)

Finally, the vital mechanism that is used in any image registration algorithm is regularization. Since image registration is inherently ill-posed, the existence and the uniqueness of the solution is not guaranteed. ${ }^{2}$ To make possible that an ill-posed problem can be turned into a wellposed problem, regularization imposes additional constraints [154]. In other words, regularization can be considered as a form of regression, which, with the purpose to avoid overfitting, constrains/regularizes or shrinks the coefficient estimates toward zero.

\subsubsection{Block Matching as Method for Piecewise Rigid Registration}

There are many intensity-based registration methods of medical images $[133,134]$. The common strategy is to assume a global relationship between the local distribution of the intensities of the images to be registered (e.g. affine, statistical, non-parametric, etc. [155]) to optimize a suitable similarity measure. In reality, such a relationship does not exist, and the linearity of most images is not compatible with how our eyes interpret them, namely in a non-linear way. Usually, light is recorded in a linear way in raw files by all digital sensors. However, as stated above,

\footnotetext{
${ }^{1}$ Other optimization algorithms include Powell's algorithm, useful up to a maximum of two parameters, and Levenberg Marquart algorithm, which becomes very slow in the case of models from ten parameters upwards [144-148].

${ }^{2}$ Hadamard $[152,153]$ claimed no ill-posed problems exist in real life, but by now it is widely acknowledged that most medical image processing problems are in fact ill-posed, see also Appendix B.1.
} 


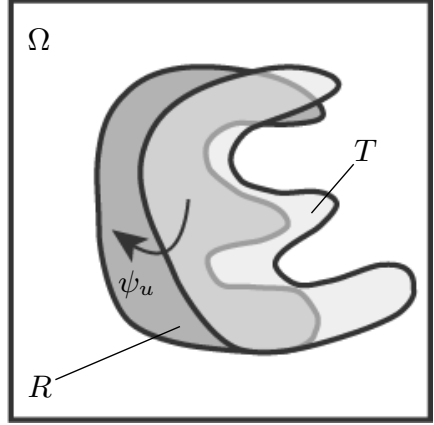

(a)

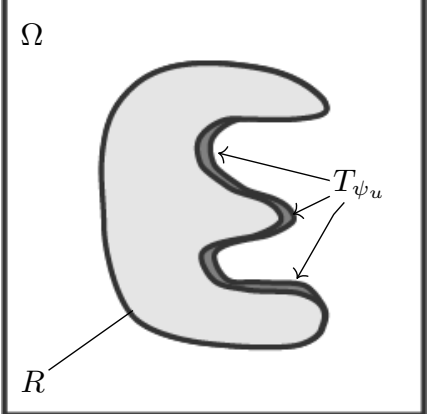

(b)

Figure 3.1.: The principle of image registration.

(a) In image registration the goal is to find a spatial transformation $\psi_{u}$ which, applied to a template image $T,(\mathrm{~b})$ produces a transformed image $T_{\psi_{u}}$ "as similar as possible" to a reference image $R$, in mathematical terms $T_{\psi_{u}}=T \circ \psi_{u} \approx R$.

the human vision is non-linear, but rather logarithmic. So, the eye expects to see something closer to a logarithmic curve ("non-linear" is commonly used to express this idea). In order to convert a recorded linear image, which for example in OCT appears very dark as raw file, back into its non-linear original version, it requires a non-linear filter. Non-linear images yield an objective function with a lot of local extrema, and thus, the optimization procedure is seldom straightforward and the intended local extrema are not always found [156].

Another problem is posed by the presence of various image artifacts, such as intensity inhomogeneities (analogously to bias field in magnetic resonance imaging, shadowing in ultrasound) and biological changes occurring in temporal studies (evolutive lesions, tumors, etc.). Artifacts can bias the assumption of global relationship criteria between the image intensities and hamper the optimization of the similarity measure [157], see Fig. 2.11.

In order to avoid undesired local extrema, we used a block matching $[157,158]$ strategy to pursue our piecewise rigid registration (see also Sec. 1.2 and 1.3). Instead of optimizing a global similarity measure with respect to the transformation parameters, block matching treats the problem in a sequential manner. The goal is to find the best position within a search region for a chosen block of pixels in a reference frame. The best position is defined by the maximum correlation between the intensities of the reference and matched block. In a first step, by comparing two frames acquired within a certain interval of time, local transformations (by individually translating and/or rotating each block) are found, which are propagated into a continuous and smooth transformation field for all the blocks involved; as a result, instead of one global maximization, several local optimizations are performed.

The region of interest, in our case the choroid-sclera interface, is subdivided into partially overlapping 3D volume blocks. This already represents a regularization in our approach. By subdividing the area into blocks, we restrict the degree of freedom of each pixel in the image in order to prevent many undesirable solutions during the optimization procedure. Our piecewise rigid registration approach can be seen as a 0-order B-spline regularization in 2D $[159,160]$ with additional restrictions of the transformation model to displacements in the $z$-direction. (Please note that this study focuses on juvenile myopia, which is primarily related to thickness changes, 
rather than structural alterations.) The blocks (of variable heights) can be considered as 0-order B-spline basis functions (with the corresponding support-size sigma variable in the $z$-direction), see Fig. 3.2. The block centers correspond to the control points. In addition to the regularization mentioned above, another one is performed to guarantee a local smoothness matching the natural shape of the eye (see. Sec. 3.3.3) which, however, is not related to the similarity measure.

After this, by regularizing the displacement field obtained, the spatial transformation is determined. This procedure is commonly applied iteratively in a multi-scale hierarchy to first recover more global and in a later stage fine and detailed deformations. The size of the neighborhood region can also be varied on a large-to-small basis.

Contrary to methods based on a global transformation, block matching provides explicit point correspondences, though very often some of them show mismatches as a result of uniformly displaced blocks or structures that are not found in the reference image. Mismatches are rejected as outliers when they are below a predefined threshold or during the robust estimation of the transformation parameters.

\subsubsection{Regularization}

As mentioned above, image registration is an ill-posed problem because there is often more than one solution (due to many local minima of the objective function), in other words more than one possible transformation to get from template to reference image. Including a regularization term in the energy functional $\mathcal{J}$ may alleviate the ill-posedness of the problem. It is the purpose of regularization to restrict the outcome of image registration by including, in form of constraints, prior knowledge of the physical properties of the underlying deformations and thus making the problem well-posed [136].

\section{$2 D$ B-Spline Regularization}

There is a myriad of different regularizations in medical image registration. In this thesis, we mainly focus on B-spline based regularization techniques. B-splines ("basis splines") are piecewise defined polynomials which are smoothly connected at their joining points ("knots"). The main advantage of using B-splines in image registration is that the resulting smoothed deformation field, generated by linear combinations of basic functions, well suited for modeling deformations in real life. In order to detect meaningful anatomical changes, such smooth transformations are often necessary. Unlike other smoothing kernels, such as the Gaussian kernel, B-spline kernels have compact, finite support, so the computational effort is considerably reduced, especially when dealing with large images. B-splines have the local approximation power of polynomials. Therefore, a high accuracy can be achieved with relatively few basic functions.

Of particular interest for the present work are the 2D B-spline basis functions third order, which are defined as the tensor product of two 1D B-spline functions (along the $x$ - and $y$-coordinate axes) of the same order and centered at each control point:

$$
K\left(p_{i}^{s}, p_{j}^{t}\right)=\beta_{3}\left(\left\|x_{i}^{s}-x_{j}^{t}\right\| / \sigma_{x}\right) \cdot \beta_{3}\left(\left\|y_{i}^{s}-y_{j}^{t}\right\| / \sigma_{y}\right)
$$

These $1 \mathrm{D}$ symmetric $\mathcal{C}^{2}\left(\Omega, \mathbb{R}^{3}\right)$, i.e. twice continuously differentiable, third-order piecewise polynomial functions, called radial cubic B-splines [161], are defined as follows: 


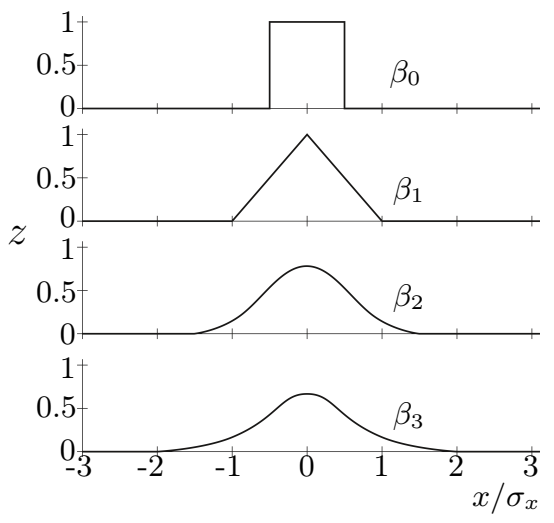

(a)

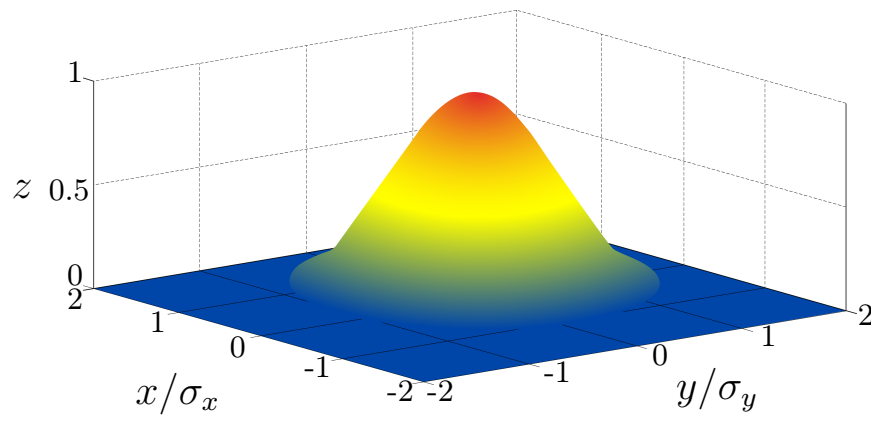

(b)

Figure 3.2.: The B-spline basis functions used.

(a) 1D B-spline basis functions of order 0 (constant), 1 (linear), 2 (quadratic) and 3 (cubic) for uniform control vector. (b) The 2D cubic B-spline kernel function. Here, $\sigma_{x}$ and $\sigma_{y}$ denote the pixel spacing in $x$ - and $y$-direction, respectively.

$$
\beta_{3}(r)= \begin{cases}2 / 3-\|r\|^{2}+\|r\|^{3} / 2 & \text { if } \quad 0 \leq\|r\|<\sigma \\ (2-\|r\|)^{3} / 6 & \text { if } \quad \sigma \leq\|r\|<2 \sigma, \text { where } r \in\left\{r_{x}, r_{y}\right\}, \sigma \in\left\{\sigma_{x}, \sigma_{y}\right\} . \\ 0 & \text { if } \quad 2 \sigma \leq\|r\|\end{cases}
$$

Furthermore, $r_{x}=\left\|x_{i}^{s}-x_{j}^{t}\right\| / \sigma_{x}$ and $r_{y}=\left\|y_{i}^{s}-y_{j}^{t}\right\| / \sigma_{y}$. Here, $\sigma_{x}$ and $\sigma_{y}$ denote the pixel spacing in $x$ - and $y$-direction, respectively (see Fig. 3.2 and Fig. 3.4).

\subsection{The Registration Problem in Mathematical Terms}

\subsubsection{The Variational Approach: Getting Low on Energy.}

In medical image analysis, the calculus of variations has been established as a helpful tool for image segmentation or registration problems. This is the field of functional analysis ${ }^{3}$ that focuses on variations, i.e. small changes in functionals, for optimization procedures. Functionals are often formulated as definite integrals involving functions and their derivatives, and, since they link functions to scalars, basically, functionals are just "functions of functions."4

Variational methods in medical imaging research are based on the principle that all things in

${ }^{3}$ Functional analysis, i.e. the branch of mathematical analysis dealing with functionals, emerged as a distinct field in the 20th century, when it was realized that diverse mathematical processes, from arithmetic to calculus procedures, exhibit very similar properties. Some of the most prominent voices in this discipline are David Hilbert (1862-1943), Stefan Banach (1892-1945), Leonhard Euler (1707-1783) and Joseph-Louis Lagrange (1736-1813) (source: https://www.britannica.com/science/functional-analysis-mathematics.)

${ }^{4} \mathrm{~A}$ simple example of such a problem is to find the curve of shortest length connecting two points. If there are no constraints, the solution is obviously a straight line between the points. However, if the curve is constrained to lie on a surface in space, then the solution is less obvious, and possibly many solutions may exist. Such solutions are known as geodesics. 
nature seek out a lower energy state. Quite different from what is the case in Physics, the word "energy" is used here as a synonym of "measure of error" or "loss" or "distance from ideal". The goal of the variational approach is to construct a functional, even called "energy functional", that aims to capture the optimal solution for a minimization problem of the "energy" value, posed, for example, by pattern recognition, or an image segmentation/registration task. If energy can be expressed as a continuous and differentiable functional, then, we have different ways of reaching the locally best value, for example by performing gradient-descent optimization, i.e. by iteratively making small steps along the negative direction of the gradient of the energy function.

\subsubsection{General Remarks}

A reference $I_{R}$ and a template image $I_{T}: \Omega \rightarrow \mathbb{R}^{+}$mapping $\Omega \subset \mathbb{R}^{3}$ to the corresponding intensities are given. Let $\mathcal{U}=\left\{\psi_{u} \mid \psi_{u}: \Omega \rightarrow \mathbb{R}^{3}, \psi_{u} \in \mathcal{C}^{2}\left(\Omega, \mathbb{R}^{3}\right), \Omega \subset \mathbb{R}^{3}\right\}$ be the set of all possible, at least twice continuously differentiable transformations mapping the domain $\Omega$ into $\mathbb{R}^{3}$. Here, $u: \Omega \rightarrow \mathbb{R}^{3}$ denote the displacement field and $u(p) \in \mathbb{R}^{3}$ is the displacement vector of $p$. For finding a transformation $\psi_{u}(p)=p+u(p), \forall p \in \Omega$, which registers $I_{R}$ and $I_{T}$, a matching criterion between the two images is needed. Such a criterion is quantified in terms of a distance function $\mathcal{D}\left[I_{R}, I_{T}, \psi_{u}\right]$, which measures the similarity between the transformed template $I_{T} \circ \psi_{u}$ and the reference image $I_{R}$ ("o" denotes the composition operator). This measure can be expressed by a loss function $\mathcal{L}: \mathbb{R} \times \mathbb{R} \rightarrow \mathbb{R}$, which determines which kind of metric is used. By integrating over the full domain $\Omega$, the corresponding similarity measure becomes

$$
\mathcal{D}\left[I_{R}, I_{T}, \psi_{u}\right]:=\int_{\hat{\Omega}} \mathcal{L}\left(I_{R}, I_{T} \circ \psi_{u}(p)\right) d p,
$$

where $\hat{\Omega}$ denotes the overlapping domain between reference $I_{R}$ and transformed template image $I_{T} \circ \psi_{u}$. The loss functions used in our work are the sum of squared differences (SSD) and the (inverse) normalized cross correlation (NCC), which we will define below (For detailed calculations of their derivatives see Appendix B.2).

In the method presented, we formulate the image registration problem in terms of the calculus of variations by considering image registration as a regularized minimization problem for the energy functional $\mathcal{J}$, according to Eq. (3.2).

$$
\underset{\psi_{u} \in \mathcal{U}}{\arg \min } \mathcal{J}\left[\psi_{u}\right], \quad \mathcal{J}\left[\psi_{u}\right]:=\mathcal{D}\left[I_{R}, I_{T}, \psi_{u}\right]+\lambda \mathcal{R}\left[\psi_{u}\right]
$$

Minimizing $\mathcal{D}\left[I_{R}, I_{T}, \psi_{u}\right]$ with respect to $\psi_{u}$ in many cases is an ill-posed problem, since no unique minimum exists. Introducing constraints (e.g. some regularity or smoothness of the displacement field) is an approach, in which undesirable solutions can be excluded, so that the problem gets well-posed. Therefore, a regularizer $\mathcal{R}$ is incorporated into the functional $\mathcal{J}$ of Eq. (3.2), which additionally measures how well the current solution fits the prior assumption about the space of admissible deformations $\mathcal{U}$. The trade-off between the terms $\mathcal{D}$ and $\mathcal{R}$ is controlled by the parameter $\lambda$. In other words, the regularization term quantifies the reasonability of the transformation and can also be used in an attempt to get closer to a mathematically sound formulation and to favor solutions that are realistic for the application in mind. 


\subsection{Mathematical Model of CRAR}

\subsubsection{The Main Idea}

In CRAR, we use [28] a piecewise rigid strategy [162] in order to globally model the deformation field of the soft choroid in an elastic way and still preserve the more rigid characteristics of the surrounding sclera and retina. In an initialization step, the slices of the reference and template stack $I_{R}, I_{T}: \Omega \subset \mathbb{R}^{3} \rightarrow \mathbb{R}^{+}$images are rigidly registered pairwise at Bruch's membrane (BM), as shown in Fig. 3.3. With the help of a graph search-based algorithm [163], BM is accurately segmented and used as shape-reference for the choroid-sclera interface (CSI) to initialize CRAR. (For details about BM and CSI we refer the reader to Sec. 2.1.4 as well as Fig. 2.8 and Fig. 2.9.) As explained in [28], this is possible as even in longitudinal studies of progressing diseases the shape of the CSI stays comparable to that of BM in its small curvature and smoothness.

Using CRAR, many problems involving unreliable segmentation can be avoided, because the exact position of the CSI is no longer needed. As a consequence, any other segmentation algorithm applied to any scan within the time sequence could be re-implemented as a starting point for comparisons.

\subsubsection{Piecewise Rigid Registration}

Let $\Omega_{j} \subset \mathbb{R}^{2}$ be the $j^{\text {th }}$ slice of size $m \times n$ pixels in a volume stack $\Omega=\bigcup_{j=1}^{S} \Omega_{j} \subset \mathbb{R}^{3}$ of $S$ slices. Utilizing BM as a reference surface as aforementioned, the volume surrounding the CSI surface is subdivided into partially overlapping $3 \mathrm{D}$ cuboidal blocks $\left\{\mathcal{C}_{i}^{s}\right\}$ (where $i=1, \ldots, N$ and $s=1, \ldots, \bar{S}$ ) defined over a regular grid of dimension $\mathcal{N}=N \times \bar{S}$ in the $O_{x y}$ plane, as illustrated in Fig. 3.4 (and, as additional visual information, see also Fig. C.2). Using a multiresolution approach, a 3D regularized block-matching registration of the CSI is conducted. As the images are aligned to the rigid BM, the displacement corresponds to shifts of the CSI. Thus, it is possible to determine the displacement field around the CSI and to use its outcome to quantify choroidal growth. Since this study focuses on quantitative choroidal thickness changes in longitudinal studies, only shifts in anterior-posterior/z-direction are considered.

In CRAR, the registration is conceived as a regularized minimization problem

$$
\underset{\psi_{u_{i}^{s}} \in \mathcal{U}}{\arg \min } \mathcal{J}\left[\psi_{u_{i}^{s}}\right], \quad \mathcal{J}\left[\psi_{u_{i}^{s}}\right]:=\mathcal{D}\left[I_{R}, I_{T}, \psi_{u_{i}^{s}}\right]+\lambda \mathcal{R}\left[\psi_{u_{i}^{s}}\right]
$$

with the aim to find, for each overlapping volume $\hat{\mathcal{C}}_{i}^{s}$ between reference and transformed template image, a set of blockwise constant transformations $\psi_{u_{i}^{s}}$ such that $I_{T} \circ \psi_{u_{i}^{s}}(p)=I_{T}\left(p+u_{i}^{s}\right) \approx I_{R}(p)$ for all $p \in \hat{\mathcal{C}}_{i}^{s}$. Recall that $\mathcal{D}$ is a distance measure that quantifies the similarity between reference $I_{R}$ and the transformed template image $I_{T}\left(p+u_{i}^{s}(p)\right)$. The regularizer $\mathcal{R}$, with its corresponding trade-off parameter $\lambda>0$, ensures certain properties of the transformation.

As in the previous stage of our method [28], for the sake of clarity denoted as CRAR-v1.0, the datasets were acquired as scan-rescan, and so we opted for the $l_{2}$-norm based Sum of Squared Differences (SSD)

$$
\mathcal{D}^{S S D}\left[I_{R}, I_{T}, \psi_{u_{i}^{s}}\right]:=\sum_{s=1}^{\bar{S}} \sum_{i=1}^{N} \int_{p \in \hat{\mathcal{C}}_{i}^{s}}\left\|\left(I_{T} \circ \psi_{u_{i}^{s}}-I_{R}\right)(p)\right\|^{2} d p
$$




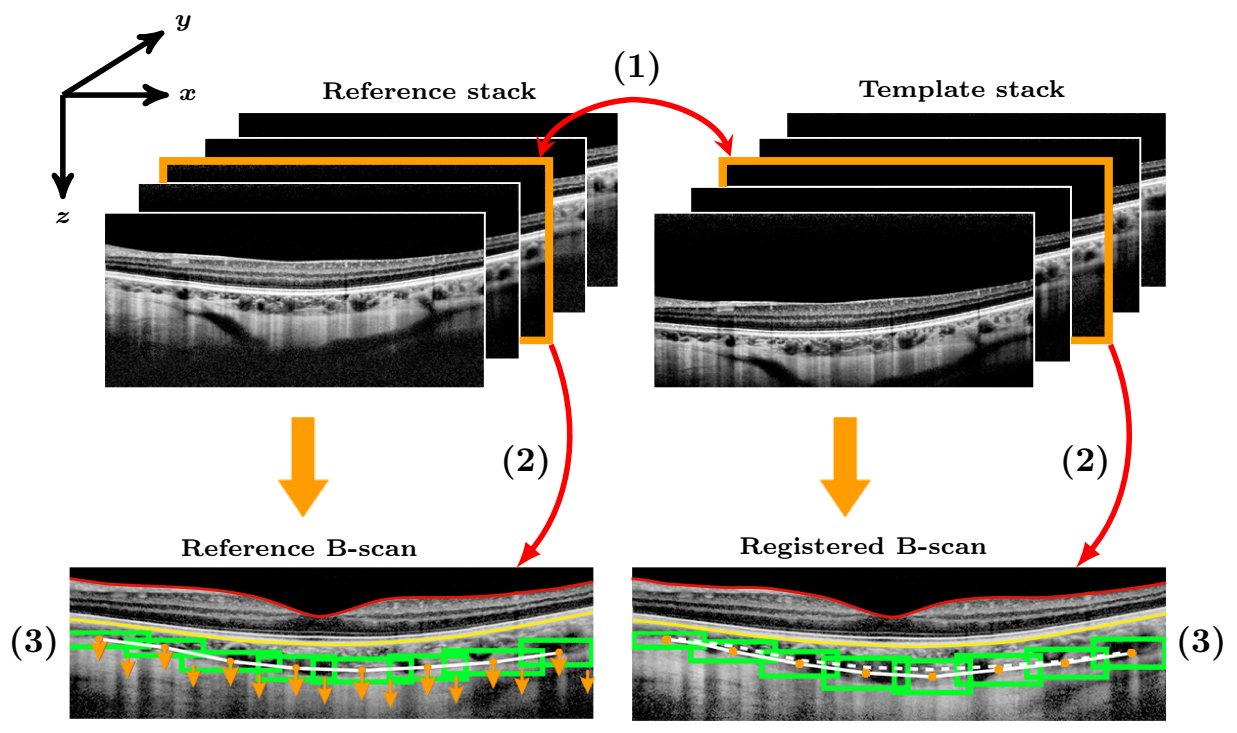

Figure 3.3.: The basic idea behind CRAR in 2D.

The pairwise inter-stack rigid registration as initialization of CRAR (1), followed by an accurate segmentation of BM (yellow line) (2). Utilizing BM as reference, the area surrounding the CSI is subdivided into partially overlapping blocks (green) (3). The displacement is represented by the shifts (short orange arrows) due to the blockwise transformation of the volume surrounding the lower boundary of the choroid.

where $p$ is the point position in $\hat{\mathcal{C}}_{i}^{s} \subset \Omega$. Here, $\hat{\mathcal{C}}_{i}^{s}$ denotes the overlapping volume between reference and transformed template image. The choice of SSD was based on the fact that with scan-rescan within a couple of minutes, images have the same intensities and are equally calibrated. In the new version of the method [164], as of now CRAR-v2.0, we analyze changes in the thickness of the choroid within time intervals of at least three months, and therefore the (inverse) Normalized Cross Correlation (NCC) is a more useful distance measure than the SSD used in CRAR-v1.0. In mathematical terms NCC is defined as

$$
\mathcal{D}^{N C C}\left[I_{R}, I_{T}, \psi_{u_{i}^{s}}\right]:=-\sum_{s=1}^{\bar{S}} \sum_{i=1}^{N} \int_{\hat{\mathcal{C}}_{i}^{s}} \frac{\sum_{p \in \hat{\mathcal{C}}_{i}^{s}}\left[I_{T} \circ \psi_{u_{i}^{s}}(p)-\mu_{T}\right]\left[I_{R}(p)-\mu_{R}\right]}{\sqrt{\sum_{p \in \hat{\mathcal{C}}_{i}^{s}}\left[I_{T} \circ \psi_{u_{i}^{s}}(p)-\mu_{T}\right]^{2}} \sqrt{\sum_{p \in \hat{\mathcal{C}}_{i}^{s}}\left[I_{R}(p)-\mu_{R}\right]^{2}}}
$$

or using the scalar product and norm notations

$$
\mathcal{D}^{N C C}\left[I_{R}, I_{T}, \psi_{u_{i}^{s}}\right]:=-\sum_{s=1}^{\bar{S}} \sum_{i=1}^{N} \int_{p \in \hat{\mathcal{C}}_{i}^{s}} \frac{\left\langle I_{T} \circ \psi_{u_{i}^{s}}(p)-\mu_{T}, I_{R}(p)-\mu_{R}\right\rangle_{\mathbb{R}^{d_{i}^{s}}}}{\left\|I_{T} \circ \psi_{u_{i}^{s}}(p)-\mu_{T}\left|\left\|_{\mathbb{R}^{d s}}|| I_{R}(p)-\mu_{R}\right\|_{\mathbb{R}^{d}}\right.\right.},
$$

where $\mu_{R}$ and $\mu_{T}$ are the average intensities of $I_{R}$ and $I_{T}$ respectively, while $\hat{\mathcal{C}}_{i}^{s}$ denotes the overlapping volume between reference and transformed template image and $p$ is the point position in block $\hat{\mathcal{C}}_{i}^{s} \subset \Omega$. Besides, $\langle\cdot, \cdot\rangle_{\mathbb{R}^{d_{i}^{s}}}$ and $\|\cdot\|_{\mathbb{R}_{i}^{d_{i}^{s}}}$ denote the scalar product and norm in $\mathbb{R}^{d_{i}^{s}}$, where $d_{i}^{s}$ corresponds to the number of voxels contained in $\hat{\mathcal{C}}_{i}^{s}$. 


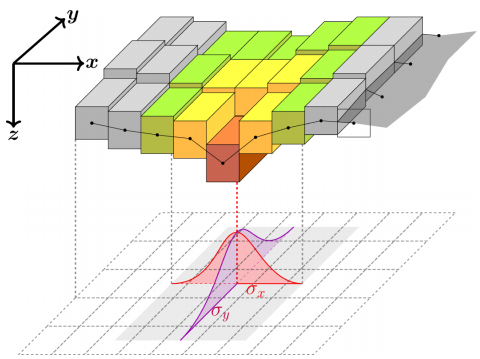

(a)

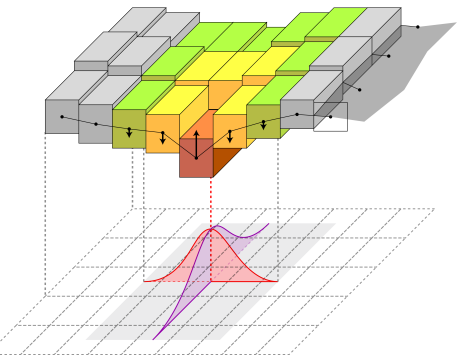

(b)

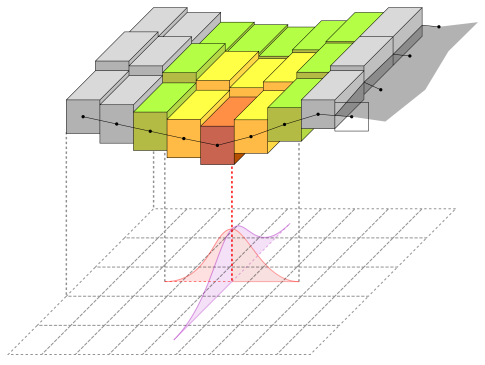

(c)

Figure 3.4.: Eye-shape adherent regularization.

(a) Block matching may leave some blocks mismatched (red). Two 1D cubic B-spline kernels with their support $2 \sigma_{x}$ and $2 \sigma_{y}$ are visualized. (b) The regularization corrects the position of the mismatched block that occurred during the $3 \mathrm{D}$ registration. This movement is counteracted by the directly neighboring blocks (yellow), which produces a smoothing effect. Blocks that are further away (light green) only have a small influence. (c) The smoothed result after eye shape adherent regularization. This matches better the physically impossible deformation of the choroid.

\subsubsection{Radial Differences Regularization}

In order to adhere to the eye's natural shape, the regularization enforces the local homogeneity of the transformations in nasal-temporal $(x-)$ and superior-inferior $(y-)$ direction by penalizing their radial differences [165]. Mismatch due to registration errors is not individually corrected. Instead, the entire neighborhood is moved until the block configuration with the least bending energy is reached, see Fig. 3.4 (and, as additional visual information, see also Fig. C.2). Let $\mathcal{N}=N \times \bar{S}$ be the total number of cuboids, $p_{i}^{s}=\left(x_{i}^{s}, y_{i}^{s}, z_{i}^{s}\right)$ and $p_{j}^{t}=\left(x_{j}^{t}, y_{j}^{t}, z_{j}^{t}\right)$ the centers of the blocks $\mathcal{C}_{i}^{s}$ and $\mathcal{C}_{j}^{t}$, respectively. Then, the regularizer $\mathcal{R}$ is defined as follows:

$$
\mathcal{R}\left[\psi_{u_{i}^{s}}\right]=\frac{1}{\mathcal{N}} \sum_{s, t=1}^{\bar{S}} \sum_{i, j=1}^{N}\left\|u_{i}^{s}\left(p_{i}^{s}\right)-u_{j}^{t}\left(p_{j}^{t}\right)\right\|^{2} \cdot K_{b}\left(p_{i}^{s}, p_{j}^{t}\right),
$$

where $u_{i}^{s}\left(p_{i}^{s}\right)$ and $u_{j}^{t}\left(p_{j}^{t}\right)$ are the corresponding displacement vectors of $p_{i}^{s}$ and $p_{j}^{t}$, as obtained from the 3D block-matching. Due to its smoothing properties and compact support, we chose the radial cubic B-spline function $K_{b}: \Omega \times \Omega \rightarrow \mathbb{R}$ as kernel. It makes sure that in case of two blocks being wide apart, displacements influence each other much less than if they are within the same $\left(2 \sigma_{x} \times 2 \sigma_{y}\right)$-neighborhood. The factor $\left\|u_{i}^{s}\left(p_{i}^{s}\right)-u_{j}^{t}\left(p_{j}^{t}\right)\right\|^{2}$ of Eq. (3.7) guarantees local homogeneity of the transformations. 


\title{
4. Intensity-based Choroidal Registration using Regularized Block-Matching
}

\begin{abstract}
In "Intensity-based Choroidal Registration using Regularized Block Matching", as of now ICR, the first version of our method is presented. Compared to the commonly used segmentation algorithms, one of the important advantages of ICR is that it works independently of any definition of the CSI, the interface separating the choroid from the sclera. The goal of ICR is to find the displacement field of the whole border area, including the CSI, separating the choroid from the adjacent sclera and to use its outcome to quantify the temporal changes in the choroidal thickness. In this prototype phase our algorithm processes scan-wise, i.e. in 2D.
\end{abstract}

\section{Publication}

The following paper was published on October $28^{\text {th }}, 2016$ in the proceedings of the Ophthalmic Medical Image Analysis, Third International Workshop (OMIA3), MICCAI 2016, Athens, Greece, pp. 33-40, [33]. It was presented on October $21^{\text {st }}, 2016$ at OMIA3, MICCAI $2016 .^{1}$

\footnotetext{
${ }^{1}$ This conference paper is available at Iowa Research Online: https://doi.org/10.17077/omia.1044.
} 
Oct 21 st, 2016

\section{Intensity-based Choroidal Registration Using Regularized Block Matching}

Tiziano Ronchetti

University of Basel

Peter Maloca

University of Basel

Christoph Meier

Bern University of Applied Sciences

Selim Orgül

University of Basel

Christoph Jud

University of Basel

See next page for additional authors

Follow this and additional works at: http://ir.uiowa.edu/omia

Part of the Ophthalmology Commons

\section{Recommended Citation}

Ronchetti, Tiziano; Maloca, Peter; Meier, Christoph; Orgül, Selim; Jud, Christoph; Hasler, Pascal; Považay, Boris; and Cattin, Philippe C.. Intensity-based Choroidal Registration Using Regularized Block Matching. In: Chen X, Garvin MK, Liu J, Trucco E, Xu Y editors. Proceedings of the Ophthalmic Medical Image Analysis Third International Workshop, OMIA 2016, Held in Conjunction with MICCAI 2016, Athens, Greece, October 21, 2016. 33-40. Available from https://doi.org/10.17077/omia.1044

Rights

Copyright $(\mathcal{C}) 2016$ the authors

Hosted by Iowa Research Online. For more information please contact: lib-ir@uiowa.edu. 
4. Intensity-based Choroidal Registration using Regularized Block-Matching

Presenter Information

Tiziano Ronchetti, Peter Maloca, Christoph Meier, Selim Orgül, Christoph Jud, Pascal Hasler, Boris Považay, and Philippe C. Cattin 


\title{
Intensity-based Choroidal Registration Using Regularized Block Matching
}

\author{
Tiziano Ronchetti ${ }^{1,2}$, Peter Maloca ${ }^{3}$, Christoph Meier ${ }^{2}$, Selim Orgül $^{3}$, \\ Christoph Jud ${ }^{1}$, Pascal Hasler ${ }^{3}$, Boris Považay ${ }^{2}$, and Philippe C. Cattin ${ }^{1}$ \\ ${ }^{1}$ Department of Biomedical Engineering (DBE), University of Basel, ${ }^{2}$ Institute for \\ Human Centered Engineering (HuCE)-optoLab, Bern University of Applied Sciences, \\ ${ }^{3}$ OCTlab, Department of Ophthalmology, University of Basel \\ Tiziano.Ronchetti@unibas.ch
}

\begin{abstract}
Detecting and monitoring changes in the human choroid play a crucial role in treating ocular diseases such as myopia. However, reliable segmentation of optical coherence tomography (OCT) images at the choroid-sclera interface (CSI) is notoriously difficult due to poor contrast, signal loss and OCT artefacts. In this paper we present blockwise registration of successive scans to improve stability also during complete loss of the CSI-signal. First, we formulated the problem as minimization of a regularized energy functional. Then, we tested our automated method for piecewise Intensity-based Choroidal rigid Registration using regularized block matching (ICR) on 20 OCT 3D-volume scan-rescan data set pairs. Finally, we used these data set pairs to determine the precision of our method, while the accuracy was determined by comparing our results with those using manually annotated scans.
\end{abstract}

Keywords: choroid-sclera, retinal layers, regularized block matching

\section{Introduction}

Myopia is a disease manifested by a disproportional growth of the eye bulb leading to a reduction and loss of visual function. It is widely diagnosed in Asian urban regions and also strongly increases in western countries. It starts to develop at school age leading to $80 \%$ incidence among teenagers. The strongly varying thickness of the choroid, the blood-filled 50 to $300 \mu \mathrm{m}$ thick layer underneath the retina, encapsulated by the rigid sclera, is correlated to not only myopia, but also to various other ocular diseases like diabetic retinopathy, intraocular tumors and macular degeneration [6]. This simple indicator is defined as the distance between the Choriocapillaris-Bruch's membrane-Retinal pigment epithelium complex (CBR) and the Choroid-Sclera Interface (CSI, see Fig. 1c). Choroidal maps (see Fig. 1b) visualize the individually and locally strongly varying thickness distribution across the area of the central choroid and can aid to extract essential clinical information. Likewise, the thickness of the retina, i.e. the distance between the Inner Limiting Membrane (ILM) and the CBR, is visualized by retinal maps (see Fig. 1a). The segmentation of the CBR is relatively

X. Chen, M. K. Garvin, J. Liu, E. Trucco, Y. Xu (Eds.): OMIA 2016, Held in Conjunction with MICCAI 2016, Athens, Greece, Iowa Research Online, pp. 33-40, 2016. Available from: http://ir.uiowa.edu/omia/2016_Proceedings/2016/ 


\section{Intensity-based Choroidal Registration using Regularized Block-Matching}

simple (e.g. using automatic segmentation methods based on graph theory [3]) because of the strong and characteristic signal of the retinal images in Optical Coherence Tomography (OCT). It has become the main contact-free, non-invasive, high-resolution imaging method that enables the detection of $\mu \mathrm{m}$ changes in the human retina and the choroid [7]. Segmenting the CSI, however, is very difficult due to the significantly lower image contrast, an increase of shadowing artefacts and unpredictable shape variations of the choroid [6]. Several studies (e.g.

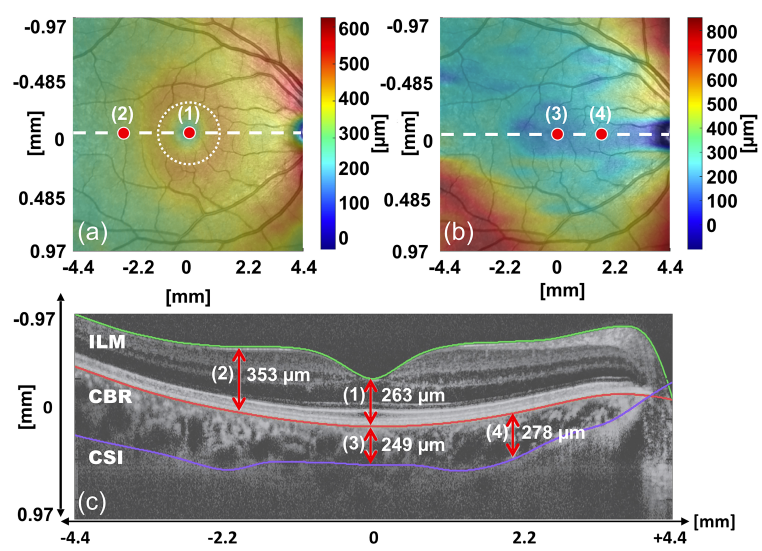

Fig. 1: (a) Retinal (ILM-CBR) and (b) choroidal (CBR-CSI) thickness maps calculated with ICR overlaid color-coded on fundus image. (c) OCT B-scan with segmented ILM, CBR and CSI layers.

$[3],[5],[9])$ use single frame segmentation, which works well in cases with good signal quality and smooth interfaces. However, this becomes difficult with less compliant subjects or in longitudinal clinical studies where successive imaging sessions can strongly vary in signal quality, leading to strong variations in image and segmentation quality [5]. Thus, reliable quantification for detection of subtle changes of choroidal thickness across a long sequence of sessions is error prone and insufficient for precise monitoring.

Instead of segmenting each tomogram individually, we suggest using image registration of image sequences obtained during a longitudinal study, utilizing the a-priori topological knowledge from previous acquisitions.

Conventional registration methods (see review article [10]) will fail when registering such OCT-data of the human eye (see Sec. 4). This is caused by the interleaved nature of the eye consisting of the soft choroid and surrounding more rigid tissues, the sclera and the CBR. It is therefore important, that sclera and $\mathrm{CBR}$ are treated as a rigid body during registration. Although a non-rigid 


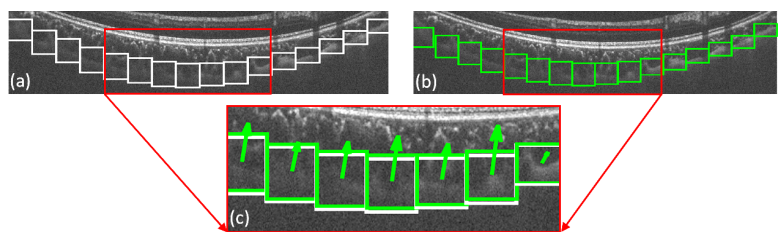

Fig. 2: Reference (a) and registered image (b) with corresponding blocks around the approximately determined CSI layer. Image (c) shows the result of the regularized block matching represented by the optimal position (green blocks) and the displacement field (green arrows) required for compensation.

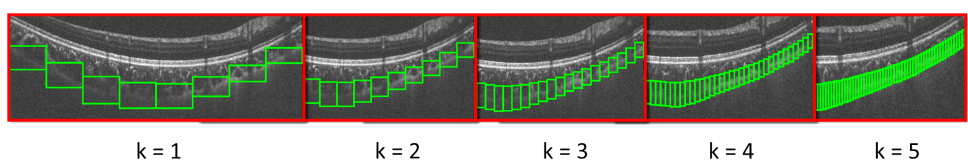

Fig. 3: Refinement of regularized block matching using different levels of subdivisions between 8 blocks $(\mathrm{k}=1)$ and 128 blocks $(\mathrm{k}=5)$.

registration procedure is imposed by the physical characteristics of the choroid, a piecewise rigid strategy [2] seems to be the most suitable and reliable approach to accurately model the deformation of the choroid. It allows us to decompose the global non-rigid matching problem of the choroid into numerous local rigid registrations of the individual subregions. The results are then embodied into a dense global non-rigid deformation field built in such a way that it elastically deforms the soft choroid and preserves the rigid characteristics of the surrounding sclera and CBR.

Our ICR method is based on the pre-segmentation of the CBR and CSI, which is tracked via registration in successive scans to quantify its growth. On the basis of the accurately segmented CBR, we approximate the position and shape of the CSI. Utilizing the rigid CBR as a reference line, we conduct hierarchical regularized block-matching [4] registration of the CSI (see Fig. 2 and 3). As the images were aligned at the rigid CBR, the displacement corresponds to either an increase or decrease of the CSI interface (see Fig. 2). This allows us to determine the displacement field in the vicinity of the CSI layer and use the outcome as a basis to quantify any choroidal growth.

\section{Material \& Method}

In this section we first introduce the image acquisition process and then we explain the processing pipeline in more detail. The OCT systems used were enhanced Spectralis OCT (Heidelberg Engineering) prototypes operating simultaneously at $800 \mathrm{~nm}$ and $1075 \mathrm{~nm}$ wavelength for obtaining different tissue contrast 


\section{Intensity-based Choroidal Registration using Regularized Block-Matching}

and penetration. The development of these dual wavelength SD-OCT system, called Hydra Spectralis, was conducted at the University of Applied Sciences in Biel, Switzerland. One of the systems is installed in the Centre for Myopia Research in Hong Kong, China, to obtain clinical data for multiple cross-sectional and longitudinal clinical studies in children and teenagers. We used 20 OCT 3D-volume data set pairs acquired as scan-rescan of 20 different Asian children between 8 and 13 years old. Ten children had their left eye and ten others had their right eye measured twice within a span of a few minutes. Each volume-

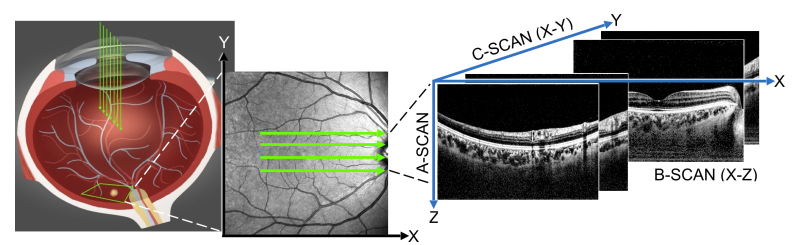

Fig. 4: The OCT scan acquisition system Hydra Spectralis.

stack consists of $25 \mathrm{~B}$-scans at a lateral resolution in nasal-temporal/ $x$-direction (see Fig. 4) was set to 768 depth-scans per frame, acquired at 20 frames/second. The axial resolution (in depth/z-direction) in the high speed acquisition mode is $11.46 \mathrm{\mu m} /$ pixel respectively $4.70 \mathrm{\mu m} /$ pixel. The scanned area is $30^{\circ} \times 20^{\circ}$ in $x$ and $y$ (including the optical nerve). Reproducibility of the scan locations is maintained by a scanning laser ophthalmoscope (SLO)-based eye-tracking system that independently raster scans the eye at $\mu \mathrm{m}$-precision and laterally repositions the OCT-beams to the target area, thereby compensating involuntary eye movement by stabilizing the $z$-position with a sturdy head-rest.

\subsection{Preprocessing}

Acquisition: A stack of up to 100 B-scans is acquired at the same position; frames are checked versus the SLO position and converted to images.

Stack registration: The acquired number of B-scans of each location and session are registered based on a similarity transformation (translation, rotation, scaling). The registration method is extended by a three-level image pyramid for multi-resolution image registration. The optimizer used for this task is a Regular Step Gradient Descent algorithm. Images that do not pass a pre-set error-level of 0.2 based on the normalized cross correlation are rejected. The registration result is a 16-bit grayscale image.

Contrast enhancement and noise reduction: To enhance the contrast, histogram equalization is applied to the original grayscale OCT image. To remove noise and locally improve the signal-to-noise ratio, Wiener filtering is slice-wise used. The cohesion of regions is improved with slice-wise median filtering. The averaged B-scans of multiple imaging sessions are combined to a new stack. 
Rigid registration of multi-session stack: Here we roughly align pairs of corresponding B-scans from the reference and floating stack images at the CBR level. To speed up this registration process we crop the images to remove the uninteresting areas with a lot of noise above the ILM and beneath the choroid. Segmentation of ILM and CBR: Using the on graph search based algorithm presented in [3], we accurately segment the ILM and the CBR. Further on the segmented CBR becomes the shape-reference for the CSI.

\subsection{Piecewise rigid registration using regularized block matching}

Let $\Omega_{j} \subset \mathbb{R}^{2}$ be the $j^{\text {th }}$ B-scans of size $m \times n$ [pixel $\left.{ }^{2}\right]$ in a volume stack $\Omega=$ $\bigcup_{j=1}^{S} \Omega_{j} \subset \mathbb{R}^{3}$. We consider a reference $I_{R}$ and a template image $I_{T}: \Omega \rightarrow \mathbb{R}$ mapping $\Omega$ to the corresponding intensities. In this context, we consider image registration as a regularized minimization problem for the energy functional $\mathcal{J}$,

$$
\min _{u} \mathcal{J}[u], \quad \mathcal{J}[u]=\mathcal{D}\left[I_{R}, I_{T}, u\right]+\lambda \mathcal{R}[u]+\mu \mathcal{S}[u] .
$$

$\mathcal{D}$ denotes a distance measure that quantifies the similarity between reference $I_{R}$ and transformed image $I_{T}(p+u(p))$. $\mathcal{R}$ and $\mathcal{S}$ are regularization terms with the corresponding balancing parameters $\lambda$ and $\mu$. They ensure certain properties of the transformation, which we will explain next.

In each B-scan we divide the interval $[0, n]$ into $N$ equidistant cells of width $\omega=\frac{n}{N}$ and cell centers $x_{i}=(2 i-1) \frac{\omega}{2}, i=1, \ldots, N$. Let $d_{i}$ be the distance between the segmented CBR and the roughly determined CSI at each location $x_{i}$, let $k_{a}$ and $k_{u}$ be two constants. We describe with $k_{a} \cdot d_{i}$ the block part above the CSI and with $k_{u} \cdot d_{i}$ the block part below. Thus, we obtain in each B-scan $\Omega_{j}$ non-overlapping rectangular blocks $\left\{\mathcal{B}_{i}^{j}\right\}_{i=1}^{N}$ with constant width $\omega$, variable height $h_{i}=\left(k_{a}+k_{u}\right) \cdot d_{i}$ and center points $p_{i}$.

The aim is to find a set $\mathcal{U}=\left\{u_{i}^{j}\right\}$ of blockwise constant transformations $u_{i}^{j} \in$ $\mathbb{R}^{3}$ such that $I_{T}\left(p+u_{i}^{j}\right) \approx I_{R}(p)$ for all $p \in \mathcal{B}_{i}^{j}$. $\mathcal{U}$ represents the displacement field between corresponding scans from the same individual.

At the first resolution level $k=1$ we initialize the block matching with 8 blocks. Applying a hierarchical strategy [1], each time we obtain $N=16,32, \ldots, 128$ blocks for every following level $k=2, \ldots, 5$ (see Fig. 3 ). Using piecewise intensitybased local rigid registration, we attempt to obtain the maximum correlation by matching blocks of the pre-determined CSI layer from the pre-registered image with the ones in the corresponding, slightly bigger search area $\hat{\mathcal{B}}_{i}^{j} \supset \mathcal{B}_{i}^{j}$.

We define the similarity measure

$$
\mathcal{D}\left[I_{R}, I_{T}, u_{i}^{j}\right]=\sum_{j=1}^{S} \sum_{i=1}^{N} \int_{p \in \mathcal{B}_{i}^{j}} \mathcal{G}_{i}(p) \mathcal{L}\left(I_{T}\left(p+u_{i}^{j}\right), I_{R}(p)\right) d p
$$

using as loss function $\mathcal{L}$ the $L_{2}$ norm $=\|\cdot\|^{2}$ :

$$
\mathcal{L}\left(I_{T}\left(p+u_{i}^{j}\right), I_{R}(p)\right)=\left\|I_{T}\left(p+u_{i}^{j}\right)-I_{R}(p)\right\|^{2},
$$




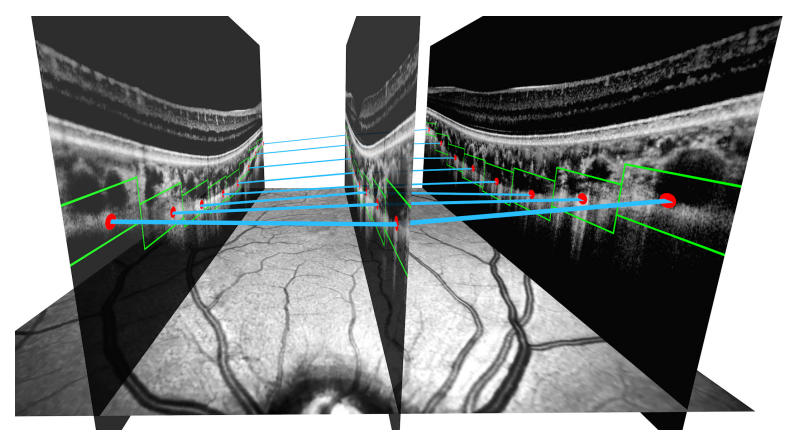

Fig. 5: With the term $S\left[u_{i}^{j}\right]$ we regularize in the scan depth across B-scans (note that the distance between the slices has been increased for better visualization). Blue lines indicate correlations between sites.

where $p$ is the point position in block $\mathcal{B}_{i}^{j} \subset \Omega_{j}$ and

$$
\mathcal{G}_{i}(p): \Omega_{j} \rightarrow \mathbb{R}_{0}^{+}, \quad \mathcal{G}_{i}(p)= \begin{cases}e^{-\left\|p-\hat{p}_{i}\right\|^{2}} & \text { if } \hat{p}=p+u_{i}^{j} \in \hat{\mathcal{B}}_{i}^{j} \\ 0 & \text { otherwise }\end{cases}
$$

is a weighting function which penalizes matches far off the target, where $\hat{p}_{i}$ is the center point of the matched block. Furthermore, we calculate for each B-scan the optimal $2^{\text {nd }}$ order polynomial which approximates the CBR-layer to obtain $\mathcal{K}$, the average of all curvatures. We use $\mathcal{K}$ as lower boundary for the regularization term $\mathcal{R}$,

$$
\mathcal{R}\left[u_{i}^{j}\right]=\sum_{j=1}^{S} \sum_{i=1}^{N}\left(\left\|D_{x}^{2}\left[u_{i}^{j}\right]\right\|-\mathcal{K}\right)^{2}
$$

where $D_{x}^{2}$ is the $2^{n d}$ order difference operator in $x$-direction. We calculate $D_{x}^{2}$ by fitting a cubic spline through the centers of the blocks and calculating its $2^{\text {nd }}$ order derivative. $\mathcal{R}$ penalizes non-smooth solutions, to avoid extreme values of the curvature of the CSI layer. In this way we regularize the vertical displacements of the blocks within a B-scan in comparison to the blocks of the corresponding reference scan. With the term $\mathcal{S}$,

$$
\mathcal{S}\left[u_{i}^{j}\right]=\sum_{j=1}^{S} \sum_{i=1}^{N}\left\|D_{y}^{2}\left[u_{i}^{j}\right]\right\|^{2}
$$

we regularize in the depth of the volume stack $\Omega$ (in $y$-direction, see Fig. 5). We calculate $D_{y}^{2}$ similarly to $D_{x}^{2}$. With this additional regularization we take into account not only the adjacent blocks within the same B-scan, but also the corresponding blocks in the neighboring scans. 
Table 1: Mean $(\bar{x})$, standard deviation $(s)$ and median $(\tilde{x})$ of the 20 data set pairs (in $[\mu \mathrm{m}]$ ) for ICR and manual (man.) registration.

\begin{tabular}{|c|c|c|c|c|c|c|c|c|c|}
\hline & \multicolumn{2}{|c|}{$\mathrm{k}=1$} & \multicolumn{2}{|c|}{$\mathrm{k}=2$} & \multicolumn{2}{|c|}{$\mathrm{k}=3$} & \multicolumn{2}{|c|}{$\mathrm{k}=4$} & $\mathrm{k}=5$ \\
\hline Tethod & ICR & man. & ICR & man. & ICR & man. & ICR & man. & ICR man. \\
\hline $\bar{x}$ & 3.52 & 6.03 & 3.62 & 7.16 & 3.92 & 8.64 & 4.15 & 10.44 & $6.81 \quad 15.96$ \\
\hline$s$ & 0.92 & 1.91 & 1.58 & 3.08 & 1.83 & 4.15 & 2.25 & 5.85 & 7.94 \\
\hline$\tilde{x}$ & 3.13 & 5.86 & 3.43 & 6.03 & 3.20 & 7.39 & 3.39 & 9.31 & $6.06 \quad 17.29$ \\
\hline
\end{tabular}

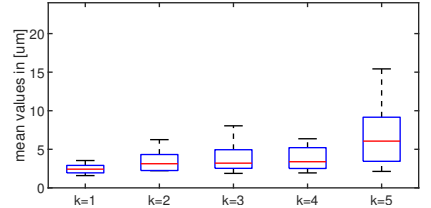

(a) Our method ICR

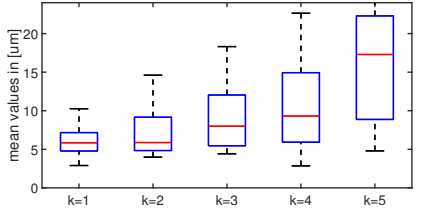

(b) Manual detection

Fig. 6: The automated (a) and the manual (b) detection of block displacements.

\section{Results}

We have tested our algorithm on the 20 data set pairs for each level $k$ and compared it to manual segmentation by a professional ophthalmologist. The results are shown in Tab. 1. Figure 6a depicts the mean values of all the block displacements in the template image during the regularized block matching for all 20 data set pairs. To get the optimal combination of precise results and a high spatial resolution, the best level of registration for our data set is $k=4$. This enables us to find choroidal changes at 64 different positions. Starting with $k=5$ a significant deterioration of the scan-rescan results can be observed. This is caused by the spatially high frequency noise of speckle that cannot be counteracted by a large structural signal in such a fine subdivision and leads to improper patch registration. Consequently, the results of the block matching algorithm become inaccurate. Figure $6 \mathrm{~b}$ shows the mean values of all the block displacements after manual segmentation of the CBR and CSI, followed by manual block building and matching (the blocks have been located by an expert as described in Sec. 2.2). A comparison of the accuracy of our method ICR and the manual detection has shown that, with increasing spatial resolution, our method still recognizes minute changes $<5 \mu \mathrm{m}$ up to the level $k=4$, which could not be attained by an ophthalmologist, even at a higher resolution.

\section{Discussion \& Outlook}

As mentioned in Sec. 1 the application of general 3D registration algorithms is in this case not useful, i.e. even the use of the smoothing parameter sigma 


\title{
4. Intensity-based Choroidal Registration using Regularized Block-Matching
}

\author{
40 T. Ronchetti et al.
}

by Demons registration algorithm [8] does not allow a satisfactory registration. With a small value of sigma the template matches the image of reference, but deformations are produced. By increasing the value of sigma, the deformations are reduced, but the images can no longer be aligned. The total time needed by our algorithm for one scan-rescan data set pair of $25 \mathrm{~B}$-scans is $295 \mathrm{~s}$ on average $^{2}$, which already makes it usable in clinical routine. We believe that an improvement in terms of computation time can be achieved with parallelization in MATLAB, followed by implementing the method in $\mathrm{C}++$. We intend to apply our method on test persons over an extended period of time and later also on patients with eye diseases. In addition, we plan to investigate the connection between the shape of the eye (curvature of the sclera) and myopia. We also consider the possibility to apply our algorithm in other fields (e.g. OCT-dermatology, ultrasound imaging).

\section{References}

1. Andronache, A., von Siebenthal, M., Székely, G., Cattin, P.: Non-rigid registration of multi-modal images using both mutual information and cross-correlation. Medical image analysis 12(1), 3-15 (2008)

2. Čech, P., Andronache, A., Wang, L., Székely, G., Cattin, P.: Piecewise rigid multimodal spine registration. In: Bildverarbeitung für die Medizin 2006, pp. 211-215. Springer (2006)

3. Chiu, S.J., Li, X.T., Nicholas, P., Toth, C.A., Izatt, J.A., Farsiu, S.: Automatic segmentation of seven retinal layers in SDOCT images congruent with expert manual segmentation. Optics express 18(18), 19413-19428 (2010)

4. De Haan, G., Biezen, P.W., Huijgen, H., Ojo, O.A.: True-motion estimation with $3 \mathrm{D}$ recursive search block matching. Circuits and Systems for Video Technology, IEEE Transactions on 3(5), 368-379 (1993)

5. Kajić, V., Esmaeelpour, M., Považay, B., Marshall, D., Rosin, P.L., Drexler, W.: Automated choroidal segmentation of $1060 \mathrm{~nm}$ OCT in healthy and pathologic eyes using a statistical model. Biomedical optics express 3(1), 86-103 (2012)

6. Morgan, I.G., Ohno-Matsui, K., Saw, S.M.: Myopia. The Lancet 379(9827), 17391748 (2012)

7. Považay, B., Hermann, B., Unterhuber, A., Hofer, B., et al.: Three-dimensional optical coherence tomography at $1050 \mathrm{~nm}$ versus $800 \mathrm{~nm}$ in retinal pathologies: enhanced performance and choroidal penetration in cataract patients. Journal of biomedical optics 12(4), 041211-041211 (2007)

8. Thirion, J.P.: Image matching as a diffusion process: an analogy with maxwell's demons. Medical image analysis 2(3), 243-260 (1998)

9. Tian, J., Marziliano, P., Baskaran, M., Tun, T.A., Aung, T.: Automatic segmentation of the choroid in enhanced depth imaging optical coherence tomography images. Biomedical optics express 4(3), 397-411 (2013)

10. Zitova, B., Flusser, J.: Image registration methods: a survey. Image and vision computing 21(11), 977-1000 (2003)

\footnotetext{
${ }^{2}$ Microsoft Windows 7 64-bit, MATLAB, R2015b, from MathWorks, Inc. (Natick, MA; US), $2.90 \mathrm{GHz}$ Intel Core i7-3520M processor with 8 GB RAM
} 


\section{Detecting Early Choroidal Changes using Piecewise Rigid Image Registration and Eye-Shape Adherent Regularization}

When we developed the first eye tracking prototype ICR (presented in Chapter 4), we mainly focused on a compactly functioning software, which brought about some limitations in how our method could be applied. In particular, ICR only worked scan-wise. That is why, in a second step, an extension to $3 \mathrm{D}$ was necessary.

With the help of the improved method "Detecting Early Choroidal Changes Using Piecewise Rigid Image Registration and Eye-Shape Adherent Regularization", as of now CRAR, we subdivide the volume around the CSI into partially overlapping cuboidal subregions, utilizing the rigid $\mathrm{BM}$ as a reference surface. ${ }^{1}$ Using a multiresolution approach, a 3D regularized block-matching registration of the CSI is conducted. As the images are aligned to the rigid BM, the displacements correspond to shifts of the CSI. Hence, it is possible to determine the displacement field around the CSI and to use its outcome to quantify local and global choroidal growth.

The regularization performed by CRAR provides an eye-shape adherent regularization, allowing for local homogeneity of the transformations. Due to its finite support, the B-spline kernel makes sure that displacements of blocks wide apart influence each other much less than if they are within the same neighborhood. Besides, the mismatched blocks of the registration process are not individually corrected, as was the case with our previous prototype ICR. Instead, the entire neighborhood is moved until the block configuration with the least bending energy is reached, which mimics the mechanical behavior of the rigid scleral tunic [166], see Sec. 2.1.1.

\section{Publication}

The following paper was published on September $9^{\text {th }}, 2017$ as a chapter in Fetal, Infant and Ophthalmic Medical Image Analysis, Lecture Notes in Computer Science (LNCS) by Springer, 2017, pp. 92-100, [28]. It was presented on September 14 ${ }^{\text {th }}, 2017$ at the Ophthalmic Medical Image Analysis, Fourth International Workshop (OMIA4), MICCAI 2017, Québec City, Québec, Canada. $^{2}$

\footnotetext{
${ }^{1}$ In our first two papers $[28,33]$ we used the CBR, the conveniently shortened form of Choriocapillaris-Bruch's membrane-Retinal epithelium complex, as reference surface instead of BM. Since the latter denotes the borderline between retina and choroid within the CBR, we switched to the by now common practice of using BM.

${ }^{2}$ This conference paper is available at Springer Professional: https://www.springerprofessional.de/detectingearly-choroidal-changes-using-piecewise-rigid-image-re/15033118.
} 


\title{
Detecting Early Choroidal Changes Using Piecewise Rigid Image Registration and Eye-Shape Adherent Regularization
}

Tiziano Ronchetti ${ }^{1,3(凶)}$, Peter Maloca ${ }^{2}$, Christoph Jud ${ }^{1}$, Christoph Meier ${ }^{3}$, Selim Orgül ${ }^{2}$, Hendrik P.N. Scholl ${ }^{2}$, Boris Považay ${ }^{3}$, and Philippe C. Cattin ${ }^{1}$

${ }^{1}$ Departments of Biomedical Engineering, University of Basel, Basel, Switzerland Tiziano.Ronchetti@unibas.ch

2 Ophthalmology, University of Basel, Basel, Switzerland

${ }^{3}$ HuCE-optoLab, Bern University of Applied Sciences, Bern, Switzerland

\begin{abstract}
Recognizing significant temporal changes in the thickness of the choroid and retina at an early stage is a crucial factor in the prevention and treatment of ocular diseases such as myopia or glaucoma. Such changes are expected to be among the first indicators of pathological manifestations and are commonly dealt using segmentation-based approaches. However, segmenting the choroid is challenging due to low contrast, loss of signal and presence of artifacts in optical coherence tomography (OCT) images. In this paper, we present a novel method for early detection of choroidal changes based on piecewise rigid image registration. In order to adhere to the eye's natural shape, the regularization enforces the local homogeneity of the transformations in nasal-temporal $(x-)$ and superior-inferior $(y-)$ direction by penalizing their radial differences. We restrict our transformation model to anterior-posterior $(z-)$ direction, as we focus on juvenile myopia, which correlates to thickness changes in the choroid rather than to structural alterations. First, the precision of the method was tested on an OCT scan-rescan data set of 62 healthy Asian children, ages 7 to 13, from a population with a high prevalence of myopia. Furthermore, the accuracy of the method in recognizing synthetically induced changes in the data set was evaluated. Finally, the results were compared to those of manually annotated scans.
\end{abstract}

Keywords: Early choroidal changes $\cdot$ Piecewise rigid registration

\section{Introduction}

The choroid is a vascular tissue located between the rather rigid sclera and retina at the posterior pole of the eye. It provides oxygen and metabolites to the retinal structures [7]. The choroid shows a thickness between 50 and $300 \mu \mathrm{m}$ with diurnal variations up to $29 \mu \mathrm{m}$. Several studies $[2,6,7]$ argue that longitudinal changes of the choroidal thickness are related to the growth of the sclera and, therefore, to the elongation of the eye bulb. Monitoring choroidal thickness delivers insight into the pathogenesis and helps in the planning of treatment of various 
ocular diseases such as myopia, glaucoma, diabetic retinopathy, choroidal tumors and macular degeneration [2]. The choroidal thickness is defined as the distance between the Choriocapillaris-Bruch's membrane-Retinal pigment epithelium complex (CBR) and the Choroid-Sclera Interface (CSI, see Fig. 1b). The common approach consists of localizing both CBR and CSI, using image segmentation $[5,9]$. To extract clinically relevant information, the distance CBR-CSI is visualized in a choroidal thickness map (see Fig. 1a). Due to the hyperreflectivity of the CBR in OCT imaging, its segmentation is relatively simple (e.g. using automatic graph-based segmentation methods [3]). In opposition, the CSI is difficult to segment because of the significantly lower image contrast, an increase of shadowing artifacts as well as unpredictable shape variations in the weakly scattering choroid [2]. The use of single frame segmentation as in [3,5,9] is difficult in longitudinal clinical studies where successive imaging sessions can strongly vary in signal quality.

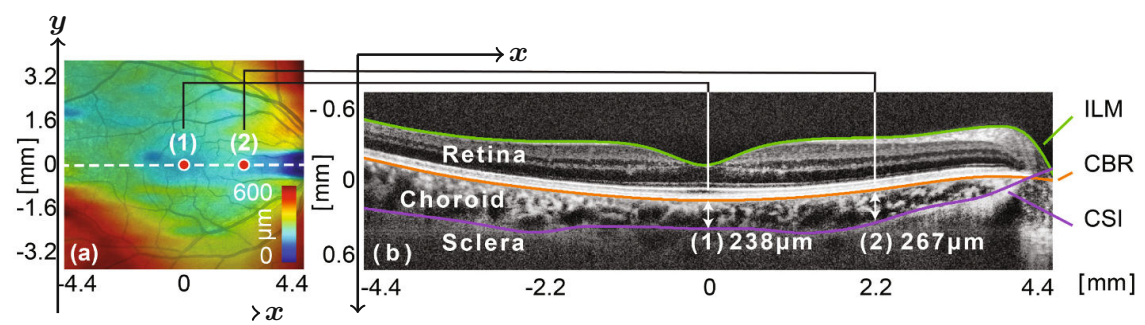

Fig. 1. (a) Choroidal (CBR-CSI) thickness map overlaid color-coded on fundus image. (b) OCT B-scan with segmented layers representing the section indicated by the white dotted line in (a). ILM is the Inner Limiting Membrane.

Due to the interleaved nature of the eye consisting of the soft choroid and surrounding more rigid tissues, the sclera and CBR, a piecewise rigid strategy [1] was used in recent developments to accurately model the deformation of the choroid. This approach allows to decompose the global non-rigid matching problem of the choroid into numerous local rigid registrations of the individual subregions. The results are embodied into a dense global non-rigid deformation field built such that it elastically deforms the soft choroid and preserves the rigid characteristics of the surrounding sclera and CBR.

In [8], the choroidal thickness changes were detected using atlas-based registration which could tackle the problem of low SNR. However, several challenges remain: (1) The method processes only scan-wise. (2) The regularization serves only as post-processing step and does not take the pixel spacing into account. (3) Due to the use of non-overlapping patches in the matching process the results become instable with increasing resolution.

In the proposed method, "Detecting early Choroidal changes using piecewise rigid image Registration and eye-shape Adherent Regularization", as of 
now CRAR, we subdivide the volume around the CSI in partially overlapping cuboidal subregions, utilizing the rigid CBR as a reference surface. Using a multiresolution approach, a 3D regularized block-matching registration of the CSI is conducted. As the images are aligned to the rigid CBR, the displacement corresponds to shifts of the CSI. Thus, it is possible to determine the displacement field around the CSI and to use its outcome to quantify choroidal growth.

This paper's contribution is threefold: (1) By grouping two or more rectangular subregions around the CSI into cuboidal blocks, the matching is done fully in 3D (see Fig. 3). (2) We simultaneously regularized along the $x$ - and $y$-axis taking different pixel spacing into account. This enforces uniform smoothness of the results matching the anatomic structure of the eye. (3) The regularization penalizes the radial differences of the transformations and favors similar displacements of patches within the same neighborhood (see Fig. 4). Mismatches are corrected during the gradient descent optimization.

In the pre-clinical experiment, we first analyzed the precision with scanrescan data. Furthermore, we showed superior change-detection performance over the CSI using smooth synthetic deformations of the rescans up to $50 \mu \mathrm{m}$ built into the data set (see Fig. 2). Finally, we compared to manual segmentation.
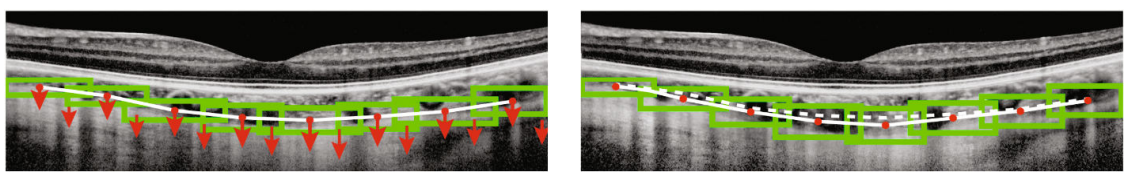

Fig. 2. Simulation of choroidal growth. Left: The white continuous line serves as reference for the deformation. Right: After blockwise transformation the reference line is shifted from the previous (white dotted line).

\section{Material and Method}

The eyes of 62 volunteers, aged 7 to 13, from Asian urban regions with a high prevalence of myopia were measured twice as scan-rescans within a few minutes, resulting in 124 OCT volume stacks. For half of them the left eye was measured, for the other the right one. For 36 of them a dual wavelength SD-OCT system prototype was used, which operated simultaneously at $800 \mathrm{~nm}$ and $1075 \mathrm{~nm}$ and was developed at the Bern University of Applied Sciences (see [8]). Each volume stack consisted of 25 slices of $768 \times 496$ pixels. The pixel spacing in nasaltemporal $/ x$ - and superior-inferior $/ y$-direction were set to $11.47 \mu \mathrm{m} / \mathrm{pixel}$ and to $245 \mu \mathrm{m} /$ pixel, the one in anterior-posterior/z-direction was set to $3.87 \mu \mathrm{m} /$ pixel. To examine the other 26 a Topcon SS-OCT system (DRI OCT Triton) was used, in which each volume stack consisted of 256 slices of $512 \times 992$ pixels. The pixel spacing in $x$-, $y$ - and $z$-direction were set to $11.72,23.44$ and to $2.60 \mu \mathrm{m} /$ pixel.

The preprocessing steps are the same as in [8]: the slices from the reference and template stack images are rigidly registered pair-wise at the CBR. Next, 


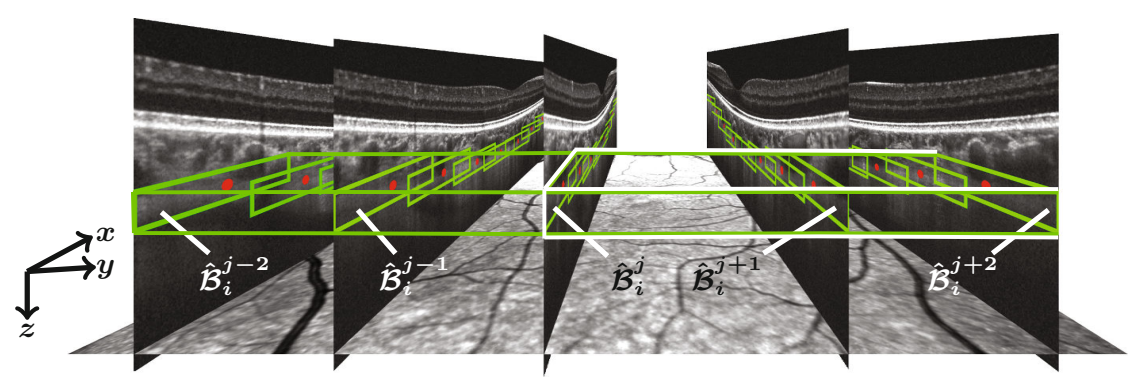

Fig. 3. The construction of the $3 \mathrm{D}$ cuboidal block $\mathcal{C}_{i}^{s}$ (green) by grouping five rectangular blocks $\hat{\mathcal{B}}_{i}^{j-2}, \hat{\mathcal{B}}_{i}^{j-1}, \hat{\mathcal{B}}_{i}^{j}, \hat{\mathcal{B}}_{i}^{j+1}$ and $\hat{\mathcal{B}}_{i}^{j+2}$ that have been normalized in height. The next block $\mathcal{C}_{i}^{s+1}$ (white) is created analogously.

using the algorithm presented in [3] based on graph search, the CBR is accurately segmented and becomes the shape-reference for the CSI.

Construction of the 3D Blocks: Let $\Omega_{j} \subset \mathbb{R}^{2}$ be the $j^{\text {th }}$ slice of size $m \times n$ pixels in a volume stack $\Omega=\bigcup_{j=1}^{S} \Omega_{j} \subset \mathbb{R}^{3}$ of $S$ slices. In each slice $\Omega_{j}$ the area around the CSI is divided into $N$ partially overlapping rectangular blocks $\mathcal{B}_{i}^{j}$. Each block has a variable height $h_{i}=\left(k_{a}+k_{u}\right) \cdot d_{i}$ and a center point $x_{i}$, where $i=1, \ldots, N$. Here, $d_{i}$ is the distance between the CBR and the roughly determined CSI at each location $x_{i}$, while $k_{a}$ and $k_{u}$ remain constant. The part of the block above the CSI is denoted by $k_{a} \cdot d_{i}$ and the part below by $k_{u} \cdot d_{i}$.

Since a high spatial resolution is needed to recognize small details, enough inner blocks are needed for a meaningful detection (the first and the last block are to be treated separately). Thus, the block matching is initialized with $N=8$ blocks with a width $\omega=\frac{m}{7(1-\phi)+1}$, where $\phi$ is the lateral overlap (in percent) between two neighboring blocks at the resolution level $k=1$. Using a multiresolution approach, the number $N$ of blocks is doubled with increasing $k$ (i.e. $\left.N=8 \cdot 2^{k-1}=2^{k+2}, k \in \mathbb{N}\right)$. To optimize the overlaps the values of $\left.\phi, \beta \in\right] 0,1[$ are selected such that, at the level $k$, the block width $\omega_{k}=\omega \cdot \beta^{k-1}$ exponentially decreases, while the percentage overlap $\phi_{k}=1-\left(\frac{m}{\omega_{k}}-1\right) /\left(2^{k+2}-1\right)$ increases.

At each $i^{\text {th }}$ position on the $x$-axis, partially overlapping $3 \mathrm{D}$ cuboidal blocks $\left\{\mathcal{C}_{i}^{s}\right\}$ are formed in the $y$-direction by normalizing the height of the rectangular blocks of two or more successive slices, and then by grouping them as follows:

$$
\mathcal{C}_{i}^{1}=\bigcup_{j=1}^{G+1} \hat{\mathcal{B}}_{i}^{j}, \quad \mathcal{C}_{i}^{s}=\bigcup_{j=1+(s-2) G}^{1+s G} \hat{\mathcal{B}}_{i}^{j}(s=2, \ldots, \bar{S}-1), \quad \mathcal{C}_{i}^{\bar{S}}=\bigcup_{j=S-G}^{S} \hat{\mathcal{B}}_{i}^{j},
$$

where $\left\{\hat{\mathcal{B}}_{i}^{j}\right\}$ are the normalized blocks and $G+1$ indicates the number of slices of two successives cuboids $C_{i}^{s}$ and $C_{i}^{s+1}$ that overlap. For example, let $G=2$ and $S=25$ be given, then, at each position $i$ on the $x$-axis $\bar{S}=13$ cuboidal blocks are built: $C_{i}^{1}$ and $C_{i}^{13}$ consist of 3 slices while $C_{i}^{2} \ldots, C_{i}^{12}$ of 5 slices (see Fig. 3 ). Between the slices, cubic interpolation is used. As a result, $\bar{S}=\left\lceil\frac{S}{G}\right\rceil$ cuboidal 
blocks in the depth of the volume stack are obtained at the $i^{\text {th }}$ position on the $x$-axis. For each cuboid $\mathcal{C}_{i}^{s}$, the upper coordinate is defined as the minimum, the lower one as the maximum of all $z$ values of the not yet normalized blocks $\mathcal{B}_{i}^{j}$.

3D Piecewise Registration: A reference $I_{R}$ and a template image $I_{T}: \Omega \rightarrow \mathbb{R}$ mapping $\Omega$ to the corresponding intensities are given. Since this study focuses on quantitative choroidal thickness changes, only shifts in axial/z-direction are considered. The aim is to find a set $\mathcal{U}=\left\{u_{i}^{s}\right\}$ of blockwise constant transformations in $z$-direction $u_{i}^{s}: \mathcal{C}_{i}^{s} \rightarrow \mathbb{R}^{3}$ such that $I_{T}\left(p+u_{i}^{s}(p)\right) \approx I_{R}(p)$ for all $p \in \mathcal{C}_{i}^{s}$. Thus, the displacement vectors are $u_{i}^{s}(p)=u_{i}^{s}\left(p_{i}^{s}\right) \in \mathbb{R}^{3}$, for all $p \in \mathcal{C}_{i}^{s}$, in which $u_{i}^{s}\left(p_{i}^{s}\right)$ is the center displacement of the block $\mathcal{C}_{i}^{s}$. We consider image registration as a regularized minimization problem for the energy functional $\mathcal{J}$,

$$
\arg \min \mathcal{U}[\mathcal{U}], \quad \mathcal{J}[\mathcal{U}]:=\mathcal{D}\left[I_{R}, I_{T}, \mathcal{U}\right]+\lambda \mathcal{R}[\mathcal{U}]
$$

$\mathcal{D}$ is a distance measure that quantifies the similarity between reference $I_{R}$ and the transformed template image $I_{T}\left(p+u_{i}^{s}(p)\right)$. The regularizer $\mathcal{R}$, with its corresponding trade-off parameter $\lambda>0$, ensures certain properties of the transformation, which will be explained next. In order to indicate how well the transformed template image $I_{T}\left(p+u_{i}^{s}(p)\right)$ matches the reference $I_{R}$, the similarity measure

$$
\mathcal{D}\left[I_{R}, I_{T}, \mathcal{U}\right]:=\sum_{s=1}^{\bar{S}} \sum_{i=1}^{N} \int_{p \in \mathcal{C}_{i}^{s}}\left\|I_{T}\left(p+u_{i}^{s}(p)\right)-I_{R}(p)\right\|^{2} d p
$$

is defined, where $p$ is the point position in block $\mathcal{C}_{i}^{s} \subset \Omega$. Using piecewise intensity-based locally rigid registration, an attempt is made to obtain the maximum correlation. This is done by matching blocks around the approximated CSI of the pre-registered image with the ones in the corresponding, slightly bigger search area $\hat{\mathcal{C}}_{i}^{s} \supset \mathcal{C}_{i}^{s}$ of the reference image.

Radial Differences Regularization: The regularizer $\mathcal{R}$ of Eq. (1) is defined by the fact that even in longitudinal studies of progressing diseases the shape of the CSI stays comparable to that of the CBR in small curvature and smoothness. Inspired by that, the regularization is defined as follows: (1) Neighboring displacements should be locally homogeneous. In other words, block centers within the same neighborhood should show a similar displacement. (2) To limit the influence of the displacement of a block to its own neighborhood, $\mathcal{R}$ ensures that the shifts of blocks further away converge to zero (see Fig. 4).

We therefore opted for a regularization based on radial differences [4] with a compactly supported radial kernel. Let $\mathcal{N}=N \cdot \bar{S}$ be the total number of cuboids, $p_{i}^{s}=\left(x_{i}^{s}, y_{i}^{s}, z_{i}^{s}\right)$ and $p_{j}^{t}=\left(x_{j}^{t}, y_{j}^{t}, z_{j}^{t}\right)$ the centers of the blocks $\mathcal{C}_{i}^{s}$ and $\mathcal{C}_{j}^{t}$ respectively. Then, the regularizer $\mathcal{R}$ is defined as follows:

$$
\mathcal{R}[\mathcal{U}]=\frac{1}{\mathcal{N}} \sum_{s, t=1}^{\bar{S}} \sum_{i, j=1}^{N}\left\|u_{i}^{s}\left(p_{i}^{s}\right)-u_{j}^{t}\left(p_{j}^{t}\right)\right\|^{2} K\left(p_{i}^{s}, p_{j}^{t}\right),
$$




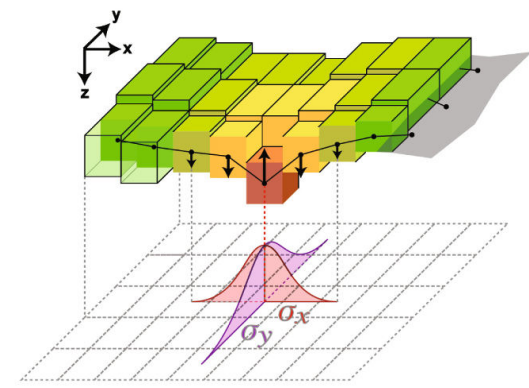

(a) The result after $3 \mathrm{D}$ block-matching

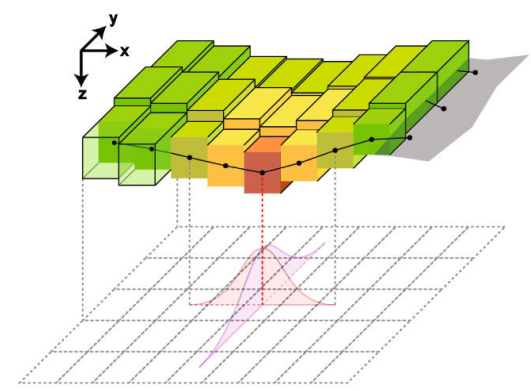

(b) The result after regularization

Fig. 4. (a) The position of the mismatched block (red) in the 3D registration is corrected by regularization. This has a smoothing effect, because the directly neighboring blocks (yellow) counteract this movement. Distant blocks (light green) have weaker influence. The blocks are drawn without overlap for better visualization. Both 1D Bspline kernels with their supports $2 \sigma_{x}$ and $2 \sigma_{y}$ are visualized underneath. (Color figure online)

where $u_{i}^{s}\left(p_{i}^{s}\right)$ and $u_{j}^{t}\left(p_{j}^{t}\right)$ are the corresponding displacement vectors of $p_{i}^{s}$ and $p_{j}^{t}$ as obtained from the 3D block-matching. Due to its smoothing properties and compact support, the radial cubic B-spline function $K: \Omega \times \Omega \rightarrow \mathbb{R}$ has been chosen as kernel. As the B-splines can be separated in single dimensions, the kernel $K$ can be split into a product of two 1D cubic B-spline kernels

$$
K\left(p_{i}^{s}, p_{j}^{t}\right)=\beta_{3}\left(\left\|x_{i}^{s}-x_{j}^{t}\right\| / \sigma_{x}\right) \cdot \beta_{3}\left(\left\|y_{i}^{s}-y_{j}^{t}\right\| / \sigma_{y}\right)
$$

where $\sigma_{x}, \sigma_{y}$ are the ratio of pixel spacing in $x$ - and $y$-direction (Fig. 4a), and

$$
\beta_{3}(r)= \begin{cases}2 / 3-\|r\|^{2}+\|r\|^{3} / 2 & \text { if } \quad 0 \leq\|r\|<\sigma \\ (2-\|r\|)^{3} / 6 & \text { if } \quad \sigma \leq\|r\|<2 \sigma, \text { where } r=\frac{\left\|x_{i}^{s}-x_{j}^{t}\right\|}{\sigma} \\ 0 & \text { if } \quad 2 \sigma \leq\|r\|\end{cases}
$$

is the $1 \mathrm{D}$ radial cubic B-spline kernel in the corresponding axis and $\sigma \in\left\{\sigma_{x}, \sigma_{y}\right\}$. Here, $2 \sigma$ describes the support in $[\mathrm{mm}]$ of the B-spline kernel in the $x$ - and $y$-direction, respectively. The factor $\left\|u_{i}^{s}\left(p_{i}^{s}\right)-u_{j}^{t}\left(p_{j}^{t}\right)\right\|^{2}$ of Eq. (2) guarantees a local homogeneity of the transformations while the B-spline kernel takes care of a $\left(2 \sigma_{x} \times 2 \sigma_{y}\right)$-neighborhood. In other words, it makes sure that in case of two blocks being wide apart, displacements influence each other much less than if they are within the same neighborhood. The mismatched blocks of the registration process are not individually corrected. Instead, the entire neighborhood is moved until the block configuration with the least bending energy is reached (Fig. 4b). 


\section{Results and Discussion}

The average displacements of all $\mathcal{N}$ blocks were detected using the proposed CRAR, a state-of-the-art method (ICR [8]) and the manual segmentation, each grouped by resolution level $k$. First, the methods were applied to the scan-rescan data set and then to the synthetically deformed data. In Fig. 5a the average displacements of the blocks from volume scan (reference) to its rescan (template) are shown. The average differences between the synthetically induced displacements (ground truth) and the measured ones are depicted in Fig. 5b.

CRAR shows, especially at higher resolution levels, a superior performance in comparison to ICR (see Fig. 5a). This is due to the fact that CRAR allows for the overlap of 3D blocks in both lateral, the $x$ - as well as the $y$-direction, whereas in ICR only non-overlapping $2 \mathrm{D}$ rectangular patches are applied. With ICR a considerable deterioration of the scan-rescan results from level $k=5$ occurs (see the trend in Fig. 5a), but with CRAR they remain precise.

This study aims at a precision in the detection of minute changes allowing clinical application: here it is set at $5 \mu \mathrm{m}$, i.e. in the range of the pixel scaling in $z$-direction. Hence, the ideal resolution level $k$ for CRAR is found at $k=7$, corresponding to the detected average displacements $|\Delta z|<2 \mu \mathrm{m}$, which is a clear improvement to ICR $(k=4,|\Delta z|>4 \mu \mathrm{m})$ and manual segmentation $(k=1,|\Delta z|>7 \mu \mathrm{m})$. This is confirmed by applying CRAR to the synthetic deformations (see Fig. 5b): CRAR remains precise with only a slightly greater variance compared to the scan-rescans. Both graphs of Fig. 5 display an important, continuous deterioration of the results using manual segmentation with increasing $k$. To penalize lower resolutions in the evaluation, the displacements at each lower level are calculated by translating the corresponding blocks in the highest resolution level $k=7$. Thus, at the $s^{t h}$ position, the displacement of the first block $\mathcal{C}_{1}^{s}$ for $k=1$ is the average of all the displacements of the centers $p_{1}^{s}, \ldots, p_{64}^{s}$ at $k=7$. For the displacement of the second block $\mathcal{C}_{2}^{s}$ at $k=1$, the average of the displacements of $p_{65}^{s}, \ldots, p_{128}^{s}$ at $k=7$ is taken. The constants $k_{a}$ and $k_{u}$ are chosen in the range of $] 0, \frac{2}{3}$ ] and ] $\left.0, \frac{1}{3}\right]$. The higher blocks include rigid

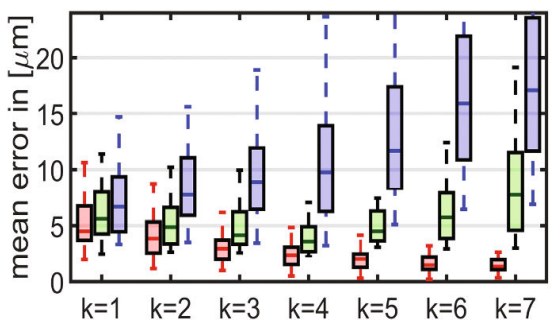

(a) Displacements by scan vs rescan

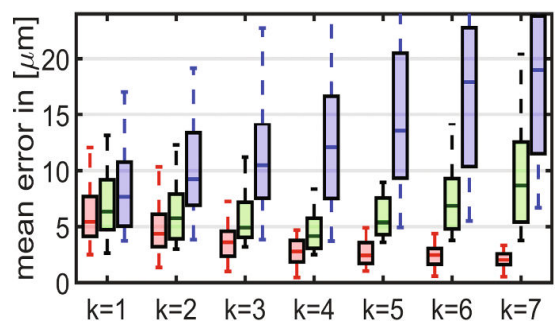

(b) Displacements by simulated changes

Fig. 5. Average displacements of the CSI obtained with CRAR (red), ICR (green), and manual expert segmentation (blue), applied on 62 OCT data set pairs (a) as scan-rescan and (b) after synthetic deformation for different $k$. (Color figure online) 
tissue, thus detectability might be reduced and subtle changes could be missed. The ideal trade-off to define the blocks was found at $\phi=0.3$ and $\beta=0.55$. After analyzing the feature density of manually segmented interfaces, the minimal needed block width for the recognition of subtle thickness changes in the choroid was set at 5 pixels. Thus, only $k \leq 7$ is examined.

\section{Conclusion}

The advantages of our method are manifold: (1) The precision in the detection of choroidal thickness changes is achieved in the range of the pixel scaling in $z$-direction. Even for finer block sizes, such as $k=5$, changes smaller than $5 \mu \mathrm{m}$ can be reliably recognized. By grouping the slices, the amount of information per patch increases, thereby reducing mismatches. (2) The regularization enforces uniform smoothness of the results matching the anatomic structure of the eye. (3) The simulated deformation proved that our method is able to detect longterm changes. (4) As the percentage overlap $\phi_{k}$ increases with $k$, the amount of information in the patches is still large, even at high resolution.

Our method, CRAR, enables unsupervised automated detection of choroidal and scleral changes and can be applied to large data sets to extract minute differences. It provides a sensitive objective progress indicator for several diseases and their respective treatments. The clinical results and interpretations of the ongoing longitudinal study of myopia development, utilizing CRAR, will be presented in an upcoming medical publication.

\section{References}

1. Čech, P., Andronache, A., Wang, L., Székely, G., Cattin, P.: Piecewise rigid multimodal spine registration. In: Handels, H., Ehrhardt, J., Horsch, A., Meinzer, H.P., Tolxdorff, T. (eds.) Bildverarbeitung für die Medizin 2006 Informatik aktuell. Springer, Heidelberg (2006). doi:10.1007/3-540-32137-3_43

2. Chhablani, J., Wong, I.Y., Kozak, I.: Choroidal imaging: a review. Saudi J. Ophthalmol. 28(2), 123-128 (2014)

3. Chiu, S.J., Li, X.T., Nicholas, P., Toth, C.A., Izatt, J.A., Farsiu, S.: Automatic segmentation of seven retinal layers in SDOCT images congruent with expert manual segmentation. Opt. Express 18(18), 19413-19428 (2010)

4. Jud, C., Möri, N., Bitterli, B., Cattin, P.C.: Bilateral regularization in reproducing kernel hilbert spaces for discontinuity preserving image registration. In: Wang, L., Adeli, E., Wang, Q., Shi, Y., Suk, H.-I. (eds.) MLMI 2016. LNCS, vol. 10019, pp. 10-17. Springer, Cham (2016). doi:10.1007/978-3-319-47157-0_2

5. Kajić, V., Esmaeelpour, M., Považay, B., Marshall, D., Rosin, P.L., Drexler, W.: Automated choroidal segmentation of $1060 \mathrm{~nm}$ OCT in healthy and pathologic eyes using a statistical model. Biomed. Opt. Express 3(1), 86-103 (2012)

6. Mutti, D.O., Gwiazda, J., Norton, T.T., Smith, E.L., Schaeffel, F., To, C.H.: Myopia yesterday, today, and tomorrow. Optom. Vis. Sci. 90(11), 1161-1164 (2013). Official publication of the American Academy of Optometry

7. Nickla, D.L., Wallman, J.: The multifunctional choroid. Prog. Retinal Eye Res. 29(2), 144-168 (2010) 
100 T. Ronchetti et al.

8. Ronchetti, T., Maloca, P., Meier, C., Orgül, S., Jud, C., Hasler, P., Považay, B., Cattin, P.C.: Intensity-based choroidal registration using regularized block matching. In: Proceedings of the Ophthalmic Medical Image Analysis Third International Workshop (OMIA), pp. 33-40 (2016)

9. Tian, J., Marziliano, P., Baskaran, M., Tun, T.A., Aung, T.: Automatic segmentation of the choroid in enhanced depth imaging optical coherence tomography images. Biomed. Opt. Express 4(3), 397-411 (2013) 


\subsection{B-Spline Deformation}

Inspired by [167], a synthetically deformed OCT B-scan was created as follows: a target thinning rate $\alpha$ was generated as a random sample from a uniform distribution between $[-25,25] \mu \mathrm{m}$, with $\alpha<0$ denoting a thinning choroid and $\alpha>0$ corresponding to a thickening one. This range for $\alpha$ was chosen to realistically represent changes that can be observed in the choroid's thickness which are bigger than the daily variations up to $29 \mu \mathrm{m}$ [168]. A 3D regular B-spline grid with $L_{1} \times L_{2} \times L_{3}$ control points was created, see Fig. 5.1. The region of interest to be manipulated was represented by two rows of grid nodes surrounding the CSI. The B-spline deformation was set to 0 outside this region.

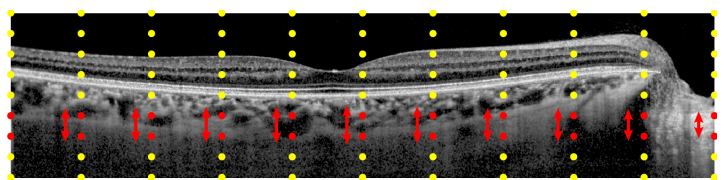

(a)

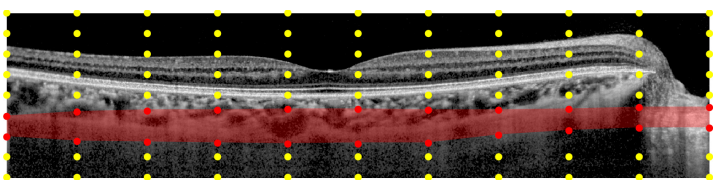

(b)

Figure 5.1.: Artificial deformation of the choroid.

(a) A regular $L_{1} \times L_{2} \times L_{3}$ grid is created. While the yellow dots remain in place, the red ones surrounding the lower boundary of the choroid are deformed. In thickening simulations (positive factor $\alpha$ ), the B-spline grid points in the choroidal region move away from each other vertically, whereas in thinning simulations (negative $\alpha$ ), they move toward each other. (b) The results after the deformation. The B-spline kernel guarantees locally smooth deformations, allowing a realistic thinning/thickening effect. 


\section{Statistical Framework for Validation without Ground Truth of Choroidal Thickness Changes Detection}

The lack of a ground truth renders validation of the detected displacements difficult. In order to prove that the results obtained are realistic, this problem must be overcome. A well-established strategy is to compare the results achieved by an automatic technique with those of a group of human experts $[169,170]$. In this paper, we first present a framework to assess the performance of CRAR purely based on common agreement, using Williams' index [171,172]. Furthermore, we discuss the validity of our results by comparing our framework with an evaluation of manual segmentations. Finally, we generate a fictitious ground truth by averaging the results of the remaining experts after a leave-one-out cross validation, and conduct an adapted power analysis to demonstrate a significantly higher performance of CRAR against the group of experts.

Publication

The following paper was published on June $28^{\text {th }}, 2019$ in PLOS ONE [164]. ${ }^{1}$

\footnotetext{
${ }^{1}$ This paper is available at PLOS: https://journals.plos.org/plosone/article?id=10.1371/journal.pone. 0218776 .
} 


\section{PLOS ONE}

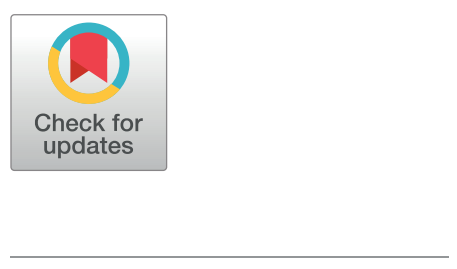

\section{G OPEN ACCESS}

Citation: Ronchetti T, Jud C, Maloca PM, Orgül S, Giger AT, Meier C, et al. (2019) Statistical framework for validation without ground truth of choroidal thickness changes detection. PLOS ONE 14(6): e0218776. https://doi.org/10.1371/journal. pone. 0218776

Editor: Sanjoy Bhattacharya, Bascom Palmer Eye Institute, UNITED STATES

Received: September 30, 2018

Accepted: June 11, 2019

Published: June 28, 2019

Copyright: ๑ 2019 Ronchetti et al. This is an open access article distributed under the terms of the Creative Commons Attribution License, which permits unrestricted use, distribution, and reproduction in any medium, provided the original author and source are credited.

Data Availability Statement: All relevant data are within the manuscript and its Supporting Information files.

Funding: This study was supported by the Swiss National Science Foundation within the SNSF Project 320030146021 "Characterization of choroidal changes in children and its temporal response to optical defocus." This work is a significant part of an international cooperation with clinical partners in Guangzhou and Hong Kong. supported by the National Natural Science
RESEARCH ARTICLE

\section{Statistical framework for validation without ground truth of choroidal thickness changes detection}

Tiziano Ronchetti $\circledast^{1,2,3 *}$, Christoph Jud ${ }^{1 \oplus}$, Peter M. Maloca ${ }^{3,4,7,8 \odot}$, Selim Orgül ${ }^{4}$, Alina T. Giger ${ }^{1}$, Christoph Meier ${ }^{2}$, Hendrik P. N. Scholl ${ }^{4,8,9}$, Rachel Ka Man Chun ${ }^{5}$, Quan Liu ${ }^{5,6}$, Chi-Ho To ${ }^{5,6}$, Boris Považay ${ }^{2 \ddagger}$, Philippe C. Cattin ${ }^{1 \ddagger}$

1 Department of Biomedical Engineering (DBE), University of Basel, Basel, Switzerland, 2 Institute for Human Centered Engineering (HuCE)-optoLab, Bern University of Applied Sciences, Bern, Switzerland, 3 OCTlab, Department of Ophthalmology, University Hospital Basel, Basel, Switzerland, 4 Department of Ophthalmology, University of Basel, Basel, Switzerland, 5 School of Optometry, The Hong Kong Polytechnic University (PolyU), Hong Kong, PR China, 6 State Key Laboratory of Ophthalmology, Zhongshan Ophthalmic Center, Sun Yat-sen University, Guangzhou, PR China, 7 Moorfields Eye Hospital, London, United Kingdom, 8 Institute of Molecular and Clinical Ophthalmology Basel (IOB), Basel, Switzerland, 9 Wilmer Eye Institute, Johns Hopkins University, Baltimore, Maryland, United States of America

- These authors contributed equally to this work.

‡ These authors also contributed equally to this work.

*Tiziano.Ronchetti@unibas.ch

\section{Abstract}

Monitoring subtle choroidal thickness changes in the human eye delivers insight into the pathogenesis of various ocular diseases such as myopia and helps planning their treatment. However, a thorough evaluation of detection-performance is challenging as a ground truth for comparison is not available. Alternatively, an artificial ground truth can be generated by averaging the manual expert segmentations. This makes the ground truth very sensitive to ambiguities due to different interpretations by the experts. In order to circumvent this limitation, we present a novel validation approach that operates independently from a ground truth and is uniquely based on the common agreement between algorithm and experts. Utilizing an appropriate index, we compare the joint agreement of several raters with the algorithm and validate it against manual expert segmentation. To illustrate this, we conduct an observational study and evaluate the results obtained using our previously published registration-based method. In addition, we present an adapted state-of-the-art evaluation method, where a paired t-test is carried out after leaving out the results of one expert at the time. Automated and manual detection were performed on a dataset of 90 OCT 3D-volume stack pairs of healthy subjects between 8 and 18 years of age from Asian urban regions with a high prevalence of myopia.

\section{Introduction}

The choroid is a vascular structure located at the posterior part of the uveal tract in the eye, between the relative rigid sclera and the more flexible, light-sensitive retina. The choroid plays 
Foundation of China NSFC, both PR China and numerous institutions in Switzerland (Basel, Bern, Luzern and Biel).

Competing interests: The authors have declared that no competing interests exist. a crucial role in the ocular metabolism circulation providing oxygen and metabolites to the outer retina $[1,2]$. Its variable thickness depends on factors such as blood pressure, axial length and age [3].

While in adults it has consistently been assessed that the choroidal thickness decreases with advancing age [4-6], studies researching choroidal development during childhood and adolescence led to contradictory conclusions. Subfoveal choroidal thickness was found to be positively correlated with age in Caucasian [7-10], but negatively in Asian children, where the prevalence of myopia is significantly higher $[5,11]$. The choroid plays an active role in emmetropization, both by modulation of its thickness to adjust the retina to the optical focus plane (choroidal accommodation) and through the regulation of the scleral growth [12, 13]. Its complex interaction with other tissues as well as its strong dependence on many other factors like blood pressure or diurnal variations, demand a precise and reliable monitoring method [1, 14].

Longitudinal studies of teenagers who develop myopia documented an eye ball elongation. This process is associated with significant thinning of the choroidal thickness in cases of high myopia [15]. Therefore, choroidal thickness, but also choroidal structure, is considered to be an important marker for monitoring myopic progression and for predicting myopia. The main challenge for detecting disease progress is to recognize particular minute changes as early as possible.

Based on optical coherence tomography (OCT) imaging [16] that unveils highly resolved details of the retina and choroid, there are segmentation- and registration-based methods for the detection of temporal changes in the thickness of the choroid (a detailed description of the different state-of-the-art methods follows below). In most cases the evaluation of such methods is performed by an artificially generated ground truth (usually the average of expert segmentations). A major drawback of such an approach is that differences between the manual segmentations cannot be correctly taken into account, when equal weights are given to all expert segmentations during averaging.

In this paper, our primary motivation is to show how to evaluate the performance of a method for choroidal thickness changes detection without generating an artificial average ground truth. We present a validation framework, purely based on common agreement, to assess the detection-performance, using the Williams' Index [17] as measure. As example of an automatic detection method we use our recently proposed registration-based method CRAR [18]. Additionally, we present an adapted state-of-the-art approach, where an artificial ground truth is created by averaging the results of the remaining experts after leaving one out at the time. In an observational study we examined long-term changes in the choroidal thickness of 90 OCT 3D-volume stack pairs of Chinese subjects between 8 and 18 years of age. For each eye, measurements were collected twice within a period of at least 3 to maximum 14 months.

Our paper's contributions are: 1) We present a statistical validation framework for automated choroidal thickness changes detection applicable in cases where a real ground truth is not available. 2) We demonstrate the framework's reliability by evaluating the results of our registration-based algorithm CRAR against those obtained by the experts. 3) We extend the commonly used power analysis approach by leave-one-out cross validation to become an ideal component of our statistical framework. 4) Based on a clinical study with volunteers with a high prevalence of juvenile myopia, we gain insight into possible correlations between time interval (between the measurements) and the choroidal thickness changes measured.

To the best of our knowledge, this is the first time that a statistical validation framework for automated choroidal thickness changes detection combines a method purely based on common agreement with an exhaustive power analysis approach. 


\section{Background and prior work}

OCT has become the main contactless, non-invasive method to characterize changes in the corneal, retinal and choroidal structures and to monitor eye growth [16]. Operating in the near infrared range, OCT provides high resolution imaging within a micrometer range and is well established in ophthalmology. Longitudinal studies using OCT imaging offer a unique possibility to gain insight into the dynamics of anatomical changes in the retina and choroid. This leads to an understanding of the mechanisms regulating such processes.

The commonly used representation of the thickness of the choroid is the choroidal thickness map, see Fig 1(a), generated from the pre-segmented data of a 3D-volume stack (C-scans) consisting of adjacent sagittal tomograms (B-scans). For every depth scan (A-scan) the difference between two segmentation-planes at this lateral location is calculated and represented. The choroidal thickness is defined as the vertical distance (along the A-scans/in $z$-direction) between the Bruch's Membrane (BM) and the Choroid-Sclera Interface (CSI), see Fig 1(b). Segmentation is frequently performed as a manual task to determine the ground truth from OCT-measurements. Due to the lack of alternative high precision in-vivo measurement methodologies, signals are typically compared to their histological equivalent. This proves to be notoriously difficult, subjective, and unreliable in view of the large amount of data points and the weak signals that are frequently hard to interpret for the human observer [14]. Even the intra-observer reproducibility is relatively low and with novel algorithms that excel in this regard a different approach to verify the reliability has to be taken. Automated detection of noisy and speckled OCT-images at the low-signal-end of the depth scan already has a long history and usually focuses on segmentation by delineating borders that are associated with large scale changes of the refractive index, or by determining tissue texture appearance.

Among the current approaches for detecting choroidal boundaries, graph search based segmentation methods represent a state-of-the-art [19-21]. However, their performance is limited by the low contrast of the choroidal boundaries, the inhomogeneity of the choroid's texture and great variation of the choroidal thickness [2]. Recently, a segmentation algorithm was presented, which combines a robust contour-detection method with a graph search, based on a novel weighting scheme [22]. However, the reliability of this method depends strongly on the choice of nodes and weights. In [23], CSI segmentation was performed using an improved graph search algorithm with curve smooth constraints. However, this approach was especially

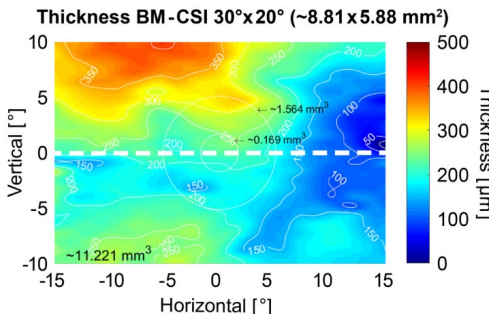

(a)

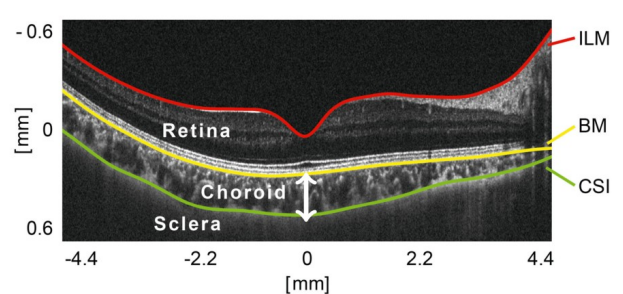

(b)

Fig 1. Choroidal thickness map and OCT B-scan with segmented layers. (a) Visualization of the choroidal thickness (BM-CSI) including the choroid's measured volume of a healthy right eye based on graph search algorithm. Circles indicate the location of the macula. The BM-CSI volume of the whole C-scan is indicated in the bottom left. (b) B-scan, a sagittal cross-section of the posterior eye segment through the retina, choroid and sclera, separated by the layers ILM, BM and CSI (source: Hydra, HuCE-optoLab/BUAS). The image was cut off in the vertical/z-direction for better visualization. The full A-scan length is $1.9 \mathrm{~mm}$. The ILM is the Inner Limiting Membrane, while BM and CSI denote the Bruch's Membrane and Choroid-Sclera Interface, respectively.

https://doi.org/10.1371/journal.pone.0218776.g001 
developed for Cirrus HD-OCT (High Definition) and still needs to be extended to other OCT devices. The use of a convolutional network architecture, where an optimal graph-edge weight can be learned directly from raw pixels, was proposed in [24]. However, this approach requires a huge amount of training data (approximately 1000 manually segmented B-scans) from the experts. In [25] the authors presented a method for unsupervised learning to identify anomalies in imaging data as candidates for pathological markers. However, it still has to be proven that the method is really able to recognize very small changes, for example in an early stage of a disease. Despite progress in image processing the use of single frame segmentation is inherently difficult, especially in longitudinal clinical studies where successive imaging sessions can strongly vary in signal quality.

In order to circumvent this limitation, we recently proposed CRAR, a method to detect early Choroidal changes using piecewise rigid image Registration and eye-shape Adherent Regularization [18]. Our method is a registration-based approach specifically developed for longitudinal studies, allowing to overcome critical problems like low contrast, loss of signal and the presence of artifacts, which are yet unsolved by most segmentation-based methods for the detection of minute choroidal changes. It needs to be emphasized that the aim of CRAR is not to localize the exact position of the CSI, i.e. the exact cutting line between choroid and sclera, but to figure out the displacement in anterior-posterior/ $z$-direction of its surrounding area within a specific time interval. Based on the resulting distortion fields, the use of a roughly localized CSI borderline is already sufficient to extract the corresponding volume changes.

In a previous paper [18] we already demonstrated CRAR's robustness regarding noise by testing the method on scan-rescans. As by scan-rescan no changes occur except the noise, the detected displacement field must be close to zero. By inducing synthetic deformations in the area of the CSI, we attested CRAR's applicability on follow-ups as well as its ability to detect changes as small as $5 \mu \mathrm{m}$ in the thickness of the choroid [18]. For more details about CRAR the reader is also referred to the S1 Appendix.

\section{Method}

To deal with the lack of valid ground truth information, we compare the results achieved by an automatic technique with those of a group of human experts, see Fig 2. In this context, one assumes that human raters hold some prior knowledge of a "mental" ground truth that is reflected in their manual tracings [26, 27]. Human rater accuracy and variability is taken into account by measuring the similarity between the expert and automatic segmentation [28]. Since a solid ground truth does not exist, the most natural solution is an evaluation based on common agreement.

The key idea is the following: we define a method $X$ to be at level with a group $\left\{Y_{i}\right\}$ of experts if $X$ agrees with each expert $Y_{i}$ at least as often as the experts $\left\{Y_{i}\right\}$ agree among themselves. In other words, with the help of an agreement index, we show that the agreement rate between algorithm $X$ and each expert is at least as high as within the expert group itself. First, we apply the similarity measures commonly used for evaluations of segmentation results. Since we are not looking for a surface but a contour (the CSI, the lower boundary of the choroid), we need a more appropriate measure for contours. To show how to proceed when working with a registration approach, where the outcome is the displacement of the contour line during the time interval between the two measurements (and not the contour itself), we use our previously published algorithm CRAR as example for method $X$. In this case, the displacement of the contour line is a very natural and intuitive metric to be used. The algorithm's performance is evaluated with an agreement test by comparing the displacements detected by CRAR with those of the experts. As an agreement measure we opt for the Williams' Index [29] 


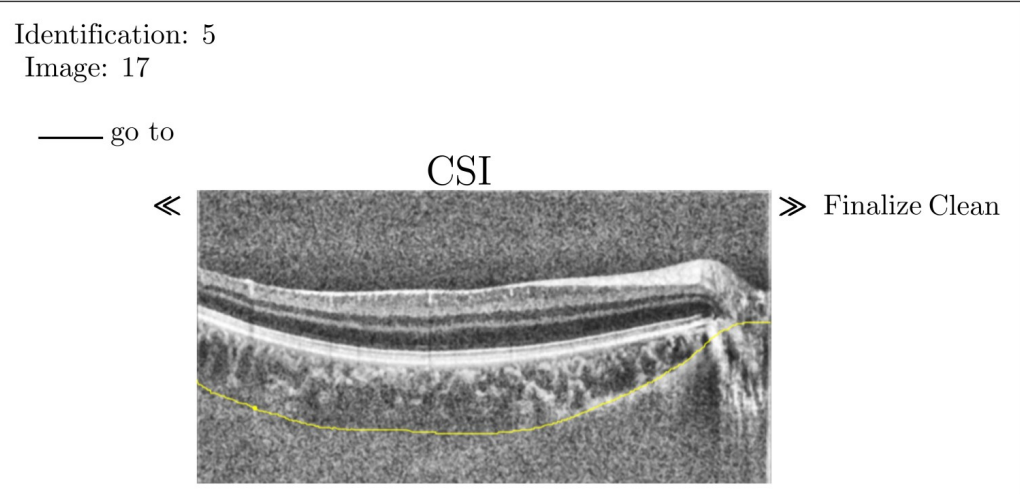

Fig 2. Sample screen of our online tool for manual expert segmentation. According to the consensus between the experts, interconnecting tissues and vessels inside the sclera were ignored while the yellow segmentation line was continued on the side of the optical nerve horizontal. The pre-processing (filtering and histogram equalization) for better contrast during the task was activated in this case by the expert.

\section{https://doi.org/10.1371/journal.pone.0218776.g002}

and demonstrate that the algorithm's performance is independent of the chosen metric. In addition, for comparison with a state-of-the-art evaluation procedure, we conduct a paired ttest using an artificially generated ground truth.

\section{Intra-rater coefficient}

In order to quantify the reliability of each expert in segmenting the same image we introduce the corresponding Intra-Rater Coefficient. Let us consider a set of $S$ images, namely OCT Bscans of size $m \times n$ pixels. Each image has to be segmented thrice by an expert. Considering the $s^{\text {th }}$ image segmented by expert $j$, the deviation $\Delta \mathcal{E}_{j}^{s}=\mathcal{E}_{j}^{s}-\overline{\mathcal{E}}_{j}^{s}$ of any one of the three segmentations $\mathcal{E}_{j}^{s}$ from their average $\overline{\mathcal{E}}_{j}^{s}$ is calculated. The number \#counts $(j, l)$ of all such deviations, which are within a given tolerance interval $[-l, l]$, i.e.

$$
\# \text { counts }(j, l)=\#\left\{\Delta \mathcal{E}_{j}^{s} \mid-l \leq \Delta \mathcal{E}_{j}^{s} \leq l\right\}, \text { where } s \in\{1, \ldots, S\},
$$

leads to the definition of the Intra-Rater Coefficient $\operatorname{IRC}_{j}$ of expert $j$

$$
\operatorname{IRC}_{j}=\frac{\# \operatorname{counts}(j, l)}{3 n S} \text {. }
$$

Here, $3 n S$ denotes the total number of pixels to be segmented by each expert and $l$ is the margin of tolerance set for the manual segmentation (in our case $l=20 \mu \mathrm{m}$ or 5 pixels).

Now, we try to get a feeling for the value of this coefficient. It can be shown that a rater that repeats the segmentation of the CSI at random will achieve an IRC of at most 0.2 . Such a rater can be simulated by inducing, in the manually annotated scans, synthetic B-spline deformations with randomly chosen coefficients for the linear combinations of their basis functions. In order to attest reliability to the manual segmentations, we aim to achieve IRC values of at least 0.70 . This value corresponds to a variance in the segmentations of one rater of approx. $25 \mu \mathrm{m}$.

\section{Williams' index}

According to Williams [17] and [29] we propose an agreement index giving an answer to the following question: given a group of $r \geq 3$ raters labeling a finite set of pixels, does rater $j$ agree 
with a group of experts in the same way as the group members agree among each other? If this is the case, the index of agreement is set equal to 1 .

Let $\mathcal{E}_{j}, \mathcal{E}_{j^{\prime}}$ and $\mathcal{E}_{j^{\prime}}$ be the results of all the manual segmentations by the experts $j, j^{\prime}$ and $j^{\prime \prime}$, the Williams' agreement Index (WI) of expert $j$ is defined as:

$$
W I_{j}=\frac{(r-2) \sum_{j^{\prime} \neq j}^{r} s\left(\mathcal{E}_{j}, \mathcal{E}_{j^{\prime}}\right)}{2 \sum_{j^{\prime} \neq j}^{r} \sum_{j^{\prime} \neq j}^{j^{\prime} \neq 1} s\left(\mathcal{E}_{j^{\prime}}, \mathcal{E}_{j^{\prime}}\right)},
$$

where $s\left(\mathcal{E}_{j}, \mathcal{E}_{j^{\prime}}\right) \in[0,1]$ provides a quantification for the similarity between the predictions of rater $j$ and $j^{\prime}$ for all pixels (for more details about $s\left(\mathcal{E}_{j}, \mathcal{E}_{j^{\prime}}\right)$ see the next subsection). The ratio derived is compared to the value of 1 . If this index is greater than 1 , it can be concluded that rater $j$ agrees with the other raters at least as often as they agree with each other [29]. Otherwise, the rate of agreement obtained between rater $j$ and the group of raters is smaller than the rate of agreement within the group of raters.

\section{Similarity measures}

The main underlying principle of our evaluation is the definition of agreement between a method $X$ (here CRAR) for automated detection of choroidal thickness changes and a group of experts doing manual segmentations. The agreement of two experts is defined as the similarity between their respective segmentations. An intuitive similarity measure for the comparison between our algorithm and manual segmentations is the difference between the automatically detected changes and the difference between manual segmentations by experts at different times. The evaluation framework is established by first calculating the WI using the common similarity measures for segmentation, i.e. the Dice Coefficient (DC) and the Jaccard Similarity Coefficient (JC). Such similarity measures are typically applied to survey the segmentation of surfaces.

As our task is to recognize the CSI, the lower contour of the choroid, we need a more appropriate similarity measure. For this reason we opt for the Bidirectional Local Distance (BLD, see Fig 3, for more detail see [30]), a more robust and conclusive similarity measure for surfaces than the DC and JC, as shown in Fig 4.

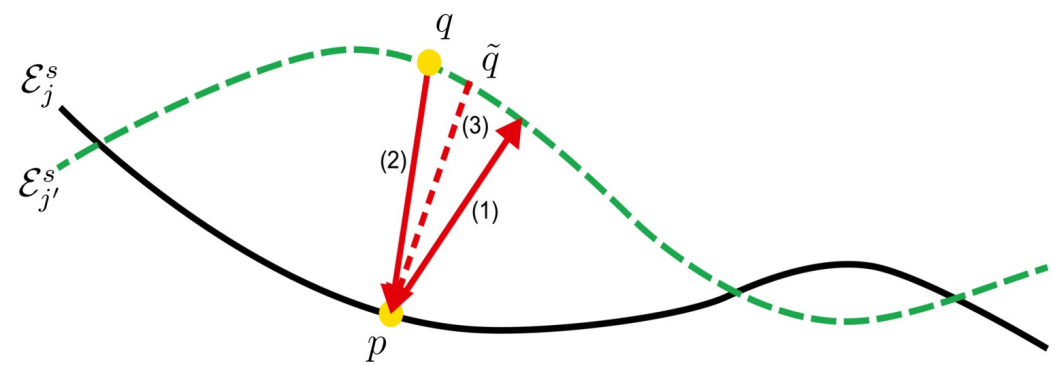

Fig 3. The calculation of the similarity measure BLD. First, the minimum "forward" distance $d_{\text {min }}\left(p, \mathcal{E}_{i^{\prime}}^{s}\right)$ between the point $p \in \mathcal{E}_{j}^{s}$ and the contour $\mathcal{E}_{j^{\prime}}^{s}$ is determined, here marked as (1). Second, among all the points $q$ on $\mathcal{E}_{j^{\prime}}^{s}$ with a "inverse" minimum distance $d_{\min }^{-1}\left(q, \mathcal{E}_{j}^{s}\right)$, those are selected whose minimal distance is found at the point $p$. Here, $q$ and $\tilde{q}$ are the candidates, with the corresponding distances denoted by (2) and (3). Then, the maximum distance among the candidates, in this case (2), is chosen as $d_{\max }^{-1}\left(\mathcal{E}_{j^{\prime}}^{s}, p\right)$. Finally, $\operatorname{BLD}\left(p, \mathcal{E}_{j^{\prime}}^{s}\right)$ is defined as the maximum between $\underline{d_{\min }}\left(p, \mathcal{E}_{j^{\prime}}^{s}\right)$ and $d_{\max }^{-1}\left(\mathcal{E}_{j^{\prime}}^{s}, p\right)$, in this example (2). For more details see [30].

https://doi.org/10.1371/journal.pone.0218776.g003 


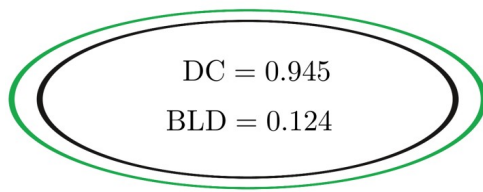

(a)

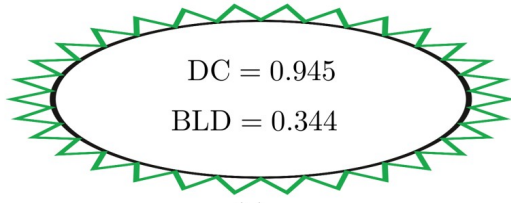

(b)

Fig 4. The robustness of the BLD in comparison to the DC for contour recognition. Here, the value of the surface delimited by the green contour is the same in both cases: (a) The region which should be recognized is an ellipse (black). (b) While the original contour was not recognized well at all, the DC for such a segmentation has yet the same high value as in (a). Using the BLD, we achieve a fairer evaluation of the segmentation, as the bad contour detection is taken into account and penalized with a higher value of the BLD (which corresponds to a minor similarity).

https://doi.org/10.1371/journal.pone.0218776.g004

Let $S$ be the number of B-scans to be segmented by each expert and let $\mathcal{E}_{j}=\left\{\mathcal{E}_{j}^{s}\right\}_{s=1}^{S}$ and $\mathcal{E}_{j^{\prime}}=\left\{\mathcal{E}_{j^{\prime}}^{s}\right\}_{s=1}^{S}$ denote the segmentations done by the experts $j$ and $j^{\prime}$ respectively. We define

$$
\operatorname{BLD}\left(\mathcal{E}_{j}, \mathcal{E}_{j^{\prime}}\right)=\sum_{s=1}^{s} \sum_{p \in \mathcal{E}_{j}^{\mathcal{E}}} \frac{\operatorname{BLD}\left(p, \mathcal{E}_{j^{\prime}}^{s}\right)}{n \max _{p \in \mathcal{E}_{j}^{s}}\left\{\operatorname{BLD}\left(p, \mathcal{E}_{j^{\prime}}^{s}\right)\right\}},
$$

where

$$
\operatorname{BLD}\left(p, \mathcal{E}_{j^{\prime}}^{s}\right)=\max \left\{d_{\min }\left(p, \mathcal{E}_{j^{\prime}}^{s}\right), d_{\max }^{-1}\left(\mathcal{E}_{j^{\prime}}^{s}, p\right)\right\},
$$

and $d_{\min }\left(p, \mathcal{E}_{j^{\prime}}^{s}\right)$ corresponds to the minimum distance from a point $p$ on the reference $\mathcal{E}_{j}$ to the test contour $\mathcal{E}_{j^{\prime}}^{s}$, while

$$
d_{\max }^{-1}\left(\mathcal{E}_{j^{\prime}}^{s}, p\right)=\max _{q \in \mathcal{E}_{j^{\prime}}^{s}}\left\{d_{\min }\left(q, \mathcal{E}_{j}^{s}\right) \mid d_{\min }^{-1}\left(q, \mathcal{E}_{j}^{s}\right)=\|q-p\|_{2}\right\}
$$

denotes the maximum inverse distance at $p$ on $\mathcal{E}_{j}^{s}$, as illustrated in Fig 3.

Although BLD is more robust than Dice and Jaccard for the detection of contours, (see Fig 4) it is, like Dice and Jaccard, not suited for the algorithm we present. As mentioned above, this algorithm is registration- but not segmentation-based, and its result is the displacement field over time of the entire border area between sclera and choroid (including the exact CSI, which is very difficult to localize). Therefore, the algorithm does not provide contours and thus, BLD, Jaccard, and Dice are not suitable as metric because we compare neither overlapping surfaces nor contours.

Consequently, we need to introduce an appropriate metric for our task, as of now diffZ (see Eq (4)) consisting of the difference between the detected displacements of the algorithm and the differences between the expert segmentations at different times, i.e.

$$
\operatorname{diffZ}\left(\Delta \mathcal{E}_{j}, \Delta \mathcal{E}_{j^{\prime}}\right)=\frac{\sum_{s=1}^{S} \sum_{i=1}^{n}\left|\Delta \mathcal{E}_{j}^{s}(i)-\Delta \mathcal{E}_{j^{\prime}}^{s}(i)\right|}{m n S},
$$

where $\Delta \mathcal{E}_{j}=\left\{\Delta \mathcal{E}_{j}^{s}\right\}_{s=1}^{S}$ and $\Delta \mathcal{E}_{j^{\prime}}=\left\{\Delta \mathcal{E}_{j^{\prime}}^{s}\right\}_{s=1}^{S}$ represent the displacements in anterior-posterior/ $z$-direction detected by the expert $j$ and $j^{\prime}$ respectively, and are computed as the difference between the segmentations of the second measurement and those of the first one. The metric diffZ provides a value between 0 and 1 , denoting the normalized difference (or, in other words, the amount of disagreement) between the detected displacements of algorithm and one expert, or, between any two experts, respectively. As a result, 0 means "no difference" (or, maximal agreement at each point) and 1 "maximum difference" (or, complete disagreement), 
respectively. The metric diffZ is especially suitable for the evaluation of registration-based algorithms such as CRAR, in which no contours are shown. At the same time, it can also be applied in longitudinal studies to evaluate segmentation-based algorithms, which aim at the segmentation of the CSI. In this case, diffZ can be defined for the algorithm as the difference between the segmentations of two measurements performed within a time interval.

\section{Power analysis (paired t-test)}

As an additional component to the presented validation framework, we now perform an extended power analysis. Let $\Delta \mathcal{E}=\left\{\Delta \mathcal{E}_{j}^{s}\right\}_{j=1, s=1}^{r, S}$ denote the total of all displacements detected by all experts. For each $j \in\{1, \ldots, r\}$ the artificial ground truth

$$
\overline{\mathcal{G}}_{j}=\frac{1}{r-1} \sum_{j^{\prime} \neq j}^{r} \Delta \mathcal{E}_{j^{\prime}}, \text { for all } \Delta \mathcal{E}_{j^{\prime}} \in \Delta \mathcal{E} \backslash\left\{\Delta \mathcal{E}_{j}\right\},
$$

is defined by leaving out the results of expert $j$ and calculating the average of the displacements detected by the remaining experts.

Let $X$ denote the displacements detected by the algorithm. For each expert $j$ a paired t-test is done to compare the errors $X_{j}=X-\overline{\mathcal{G}}_{j}$ and $Y_{j}=\Delta \mathcal{E}_{j}-\overline{\mathcal{G}}_{j}$. In other words, after defining an artificial ground truth, we compare the difference in the errors of both algorithm and experts in their detection of choroidal thickness changes. Thus, we test the null hypothesis that the pairwise differences $X_{j}-Y_{j}$ come from a normal distribution with mean equal to 0 at the $\alpha=$ 0.01 significant level. In order to reject the null hypothesis, the result of the $p$-value must be smaller than $\alpha$. While a $p$-value shows whether an effect exists, it will not reveal the size of the effect (it might be, that a smaller $p$-value has occurred only on the basis of a large sample size [31]). This is why, we report both statistical (the $p$-value) and substantive significance (effect size). Using the Cohen's distance $d$ between both datasets, the effect size can be determined by calculating the mean difference between the two groups $X_{j}$ and $Y_{j}$, and then dividing the result by the pooled standard deviation $\sqrt{\mathcal{S}}$, i.e.

$$
\text { Cohen's } d_{j}=\frac{\mu\left(X_{j}\right)-\mu\left(Y_{j}\right)}{\sqrt{\mathcal{S}}}, \quad \text { where } \mathcal{S}=\frac{\left(\left|X_{j}\right|-1\right) \cdot \sigma^{2}\left(X_{j}\right)+\left(\left|Y_{j}\right|-1\right) \cdot \sigma^{2}\left(Y_{j}\right)}{\left|X_{j}\right|+\left|Y_{j}\right|-2},
$$

where $\left|X_{j}\right|$ and $\left|Y_{j}\right|$ denote the sample sizes of $X_{j}$ and $Y_{j}$ respectively, while $\sigma\left(X_{j}\right)$ and $\sigma\left(Y_{j}\right)$ are their standard deviations. The necessity of this "leave-one-out" power analysis lies in the fact that, in our case, an artificially generated ground truth can only be represented in the form of a matrix, as to every OCT B-scan pair a corresponding ground truth for comparison has to be generated. This results for $\overline{\mathcal{G}}_{j}$ in a matrix of size $n \times S$ corresponding to the ground truth for the entire dataset. As a result, the values of the standard deviations for $X-\overline{\mathcal{G}}_{j}$ vs. $\Delta \mathcal{E}_{j}-\overline{\mathcal{G}}_{j}$ are different from those for $X$ vs. $\Delta \mathcal{E}_{j}$. Consequently, the values of the effect size, quantified by Cohen's $d$, change as well.

\section{Material}

\section{Subjects}

Ninety OCT 3D-volume stack pairs of Chinese subjects, aged 8-18 and stemming from urban regions with a high prevalence of myopia, have been analyzed. Healthy subjects with good distant and near vision (monocular corrected visual acuity was equal or better than LogMAR 0.00 ), but no systemic and ocular diseases, ocular trauma or surgery, were recruited. For the 
subjects aged $8-13$, the spherical refractive errors were $-1.00 \mathrm{D}$ to $-5.00 \mathrm{D}$ and cylindrical power was not more than $-1.50 \mathrm{D}$. For the others, the spherical refractive errors were $+0.75 \mathrm{D}$ to $-3.00 \mathrm{D}$ and the cylindrical power was not more than $-1.00 \mathrm{D}$. Written consent was obtained from both volunteers and, when necessary, their parents. The study protocol was approved by the Human Subjects Ethics Sub-committee of The Hong Kong Polytechnic University and was conducted in adherence to the tenets of the Declaration of Helsinki.

\section{Data acquisition}

The volunteers were measured twice at different times: half of them their left eye, the other half their right one. This resulted in 180 OCT volume stacks, each consisting of $25 \mathrm{~B}$-scans of $500 \times 768$ pixels. The pixel spacing in nasal-temporal $/ x$ - and superior-inferior $/ y$-direction were set to $11.46 \mu \mathrm{m} / \mathrm{pixel}$ and to $245 \mu \mathrm{m} /$ pixel respectively, the one in anterior-posterior/zdirection was set to $3.87 \mu \mathrm{m} /$ pixel. The volunteers were divided into three groups: the first group was measured a second time after 3 months, the second after 8 months, the third after 14 months.

The images were acquired by an eye-tracking dual-wavelength OCT system operating simultaneously at the 870 and $1075 \mathrm{~nm}$ bands. This system was developed at the HuCE-optoLab at the Bern University of Applied Sciences in Biel before it was transferred and setup at the Hong Kong Polytechnic University's School of Optometry. For technical details on the OCT system used we refer to the S2 Appendix.

\section{Manual expert segmentation}

Six ophthalmologists were recruited as experts. The experts received access to a Java-based online tool with which they could draw the CSI line, using either the mouse (PC) or a pen (tablet), see Fig 2. The experts could chose to segment the CSI without processing or to activate a pre-processing consisting of a histogram equalization and an average filtering. In this evaluation step the focus lay on both the intra-rater reliability of each individual expert and the agreement among the experts.

The 90 volume stack pairs were randomly distributed among the six experts, in such a way that each expert got exactly 50 volume stack pairs and each pair was assigned to at least three different experts for CSI segmentation. This allowed to test the agreement between the experts. From each volume stack pair, eight B-scan pairs were chosen for manual expert segmentation of the CSI, three in the lower (no. 1, 3 and 6), three in the middle (no. 11,13 and 16) and two in the upper region (no. 21 and 23). To test the intra-rater reliability, each expert unknowingly received three times the same scan pair. The number of lines to be segmented by each expert was 2400 ( 8 scan positions per volume stack $\times 2$ measurements per volunteer $\times 50$ volume stacks $\times 3$ repetitions). Calculating a time of approx. $9 \mathrm{sec}$ per line, this adds up to $6 \mathrm{~h}$ per expert.

\section{Manual segmentation (consensus)}

Manual segmentation was done according to the following mutual consensus: the lower border of the choroid was drawn without taking into account the interconnecting tissues that appear as humps on top of the slowly varying baseline. Also the shadow artifacts that are cast by the retinal and choroidal vasculature were ignored. Vessels inside the sclera were also disregarded. It was agreed that once the CSI came to an end near the optical nerve, the segmentation line would be continued in the same direction and with the same slope, as depicted in Fig 2. 


\section{Results and discussion}

Fig 5 shows the intra-rater reliability (repeatability) of the results of each expert by having the same image segmented thrice. The six experts together show an averaged standard deviation of $\pm 24.73 \mu \mathrm{m}$ in rating the same image. It is remarkable, that the experts are often inconclusive about the position of the CSI in the temporal para- and perifoveal region opposite to the ocular nerve, see later Fig 6(c) and 6(d). This is due to the low contrast in the image acquisition in this area of the choroid which makes a clear identification of the CSI difficult. The mean IRC value achieved by the experts was 0.807 , which is to be considered as a good result. It has to be pointed out that the lowest scores were reached by those experts who probably had experienced difficulties in handling the tool. The first version of our online tool has been continuously optimized based on the feedback of the raters. Despite the challenges, the IRC scores were almost as good as we wished for.

Fig 7 presents the values of the WI calculated between each individual expert and the other members of the group for all the images (using DC, JC, BLD and diffZ as metric). When we compare the segmentations performed by the experts, the results show an average WI of $0.9992 \pm 0.0221$. The weak scattering of the WI demonstrates that its value does not depend on the choice of the similarity measure. Fig 7 shows that there are no relevant differences between the values of the WI whether they are calculated on the base of diffZ or the other three metrics, so Dice, Jaccard and BLD are not indispensable to this kind of evaluation, and therefore no longer needed. This justifies the use of diffZ for our case even more. The comparison between

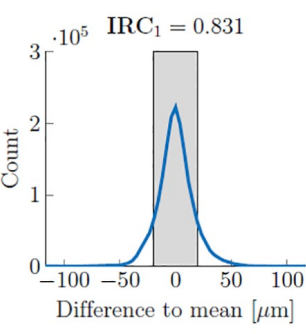

(a)

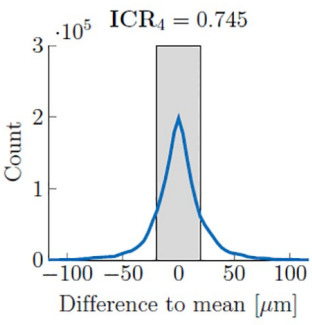

(d)

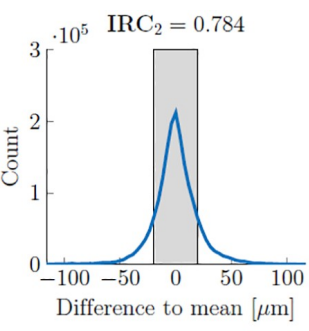

(b)

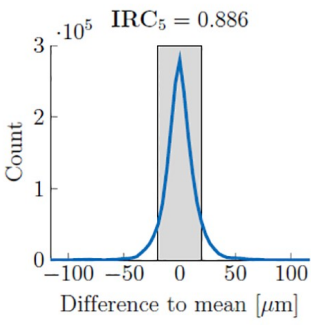

(e)

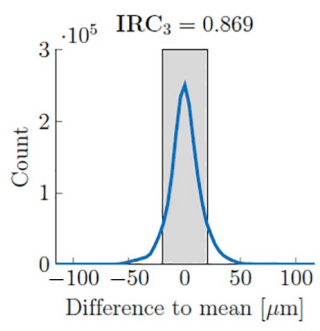

(c)

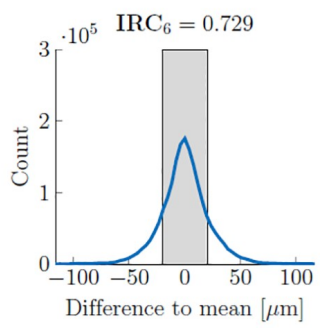

(f)

Fig 5. The representations of the intra-rater reliability of experts 1-6 ordered from (a)-(f). At every pixel position, the difference to the average value of the three available segmentations per rater is calculated. If its absolute value is smaller than a predefined threshold (here set to $20 \mu \mathrm{m}$ represented by the grey area) then it is counted, i.e. the prediction is considered reliable. Therefore, the narrower and higher the curve, the more reliable the segmentation by the corresponding expert is. The number of counts found within this range is divided by the total number of segmentation points graded by the corresponding expert. By the obtained normalized value $\mathrm{IRC}_{j}$ we define the IntraRater Coefficient to quantify the reliability of the $j^{\text {th }}$ expert.

https://doi.org/10.1371/journal.pone.0218776.g005 


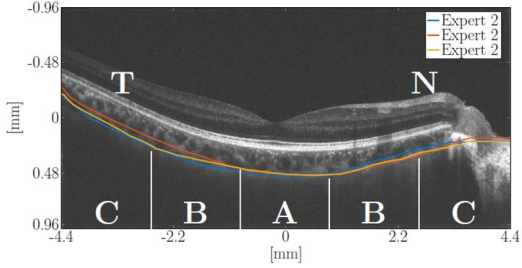

(a)

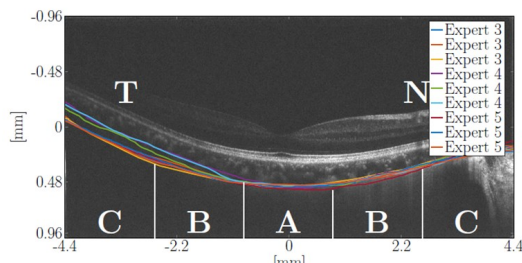

(c)

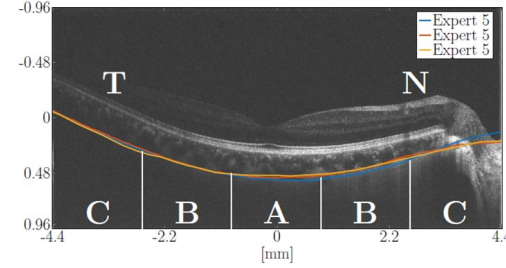

(b)

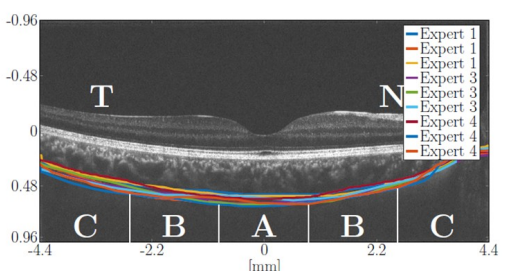

(d)

Fig 6. Examples of manual expert segmentation (consistent and less consistent with each other). Top: Repeatability of (a) expert 2 and (b) expert 5 when segmenting the CSI. Bottom: Comparison of segmentations by (c) experts 3, 4 and 5 and (d) experts 1,3 and 4 . The choroidal area is subdivided in nasal $(\mathrm{N})$-temporal $(\mathrm{T}) / x$-direction into five equidistant regions (patches) symmetrically around the foveal center: A (foveal region), B (parafoveal region), and C (perifoveal region). Here only cases of right eyes are depicted.

https://doi.org/10.1371/journal.pone.0218776.g006

CRAR and the experts group shows that CRAR's predictions match those of the experts (the WI is greater than 1). To exclude that one expert influences the value of the WI much more than another, the WI is recalculated by omitting one expert at a time. The calculation of the WI between CRAR and the remaining five experts after leaving-one-out gives values in the range of [1.0126, 1.0393].

Fig 8 shows the variability at each scan position grouped by expert. As expected, the displacements detected by CRAR $(4.29 \pm 23.73 \mu \mathrm{m}$, see the far right hand side of Fig 8$)$ are within the range of those detected by the experts $(5.47 \pm 39.45 \mu \mathrm{m})$ but with a smaller variance. This is

\begin{tabular}{|c|c|c|c|c|c|}
\hline $\operatorname{Exp} 1$ & 0.9975 & 0.9987 & 1.0638 & 1.0254 & 1.06 \\
\hline Exp 2 & 0.9989 & 0.9994 & 0.9856 & 1.0257 & \\
\hline $\operatorname{Exp} 3$ & 1.0024 & 1.0013 & 0.9192 & 0.9535 & 1.00 \\
\hline $\operatorname{Exp} 4$ & 1.0015 & 1.0008 & 0.9604 & 0.9895 & 0.98 \\
\hline $\operatorname{Exp} 5$ & 1.0001 & 1.0000 & 1.0384 & 0.9810 & 0.96 \\
\hline $\operatorname{Exp} 6$ & 0.9996 & 0.9998 & 1.0417 & 0.9974 & 0.94 \\
\hline \multirow[t]{2}{*}{ CRAR } & & & & 1.0289 & \\
\hline & Jaccard & Dice & BLD & $\operatorname{diff} Z$ & \\
\hline
\end{tabular}

Fig 7. The values of the WI calculated for the algorithm and the experts group. As similarity measures Jaccard, Dice, BLD and diffZ are used.

https://doi.org/10.1371/journal.pone.0218776.g007 


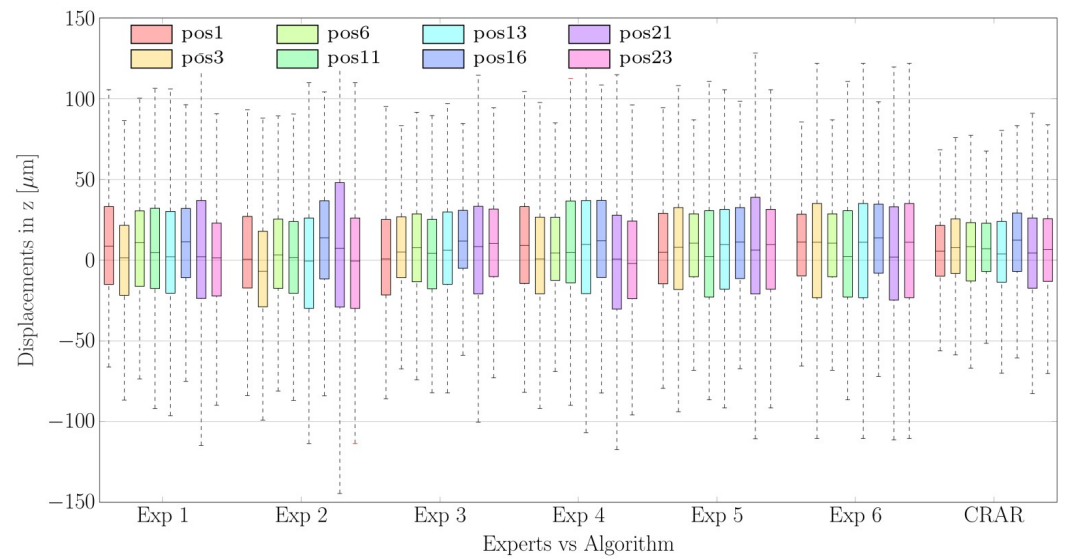

Fig 8. The average displacements of the CSI grouped by experts and algorithm. The results are obtained by manual segmentation by the six experts and by CRAR (subdivided into the B-scan positions 1, 3, 6, 11, 13, 16, 21 and 23). https://doi.org/10.1371/journal.pone.0218776.g008

due to the fact that our automated algorithm guarantees perfect repeatability $(\operatorname{IRC}=1)$, unlike the human rater.

Fig 9(a) and 9(b) show the total variability of all displacements detected by the experts and the algorithm, respectively, grouped by time interval between the two measurements. The average displacements measured using CRAR are $1.41 \pm 16.23 \mu \mathrm{m}$ in the case of images captured within a time interval of 3 months. For the other two time intervals of 8 and 14 months, the average changes are $4.88 \pm 22.63 \mu \mathrm{m}$ and $6.12 \pm 29.32 \mu \mathrm{m}$, respectively. These results are still consistent with those of the experts, i.e. $1.76 \pm 26.72 \mu \mathrm{m}, 5.67 \pm 32.48 \mu \mathrm{m}$ and $7.62 \pm$ $39.15 \mu \mathrm{m}$ but with smaller variations. These results are also summarized in Table 1 for better data visualization. During each time interval between measurements the choroidal thickness increases, as is to be expected for growing tissue. This finding supports the hypothesis which several studies [7-10] formulate: the increase in thickness of the choroid is a normal feature of eye growth from early childhood to adolescence. Such a thickening of the choroid could relate to changes in the structure and associated physiological demands of the outer retina occurring during the eye's natural development, and/or to its role as sclera growth regulator [13].

On the other hand, the thickening of the choroid seems to slow down as time goes by, while the scattering of the data increases. The slower progress in thickening could be related to the high prevalence of Chinese children to become myopic $(\approx 80 \%$ in the age $13-15)$. It could also be a sign that a disproportionate elongation of the eye-ball is taking place and must be compensated by slowing down the growth or, in case of myopia, even an actual thinning of the choroidal thickness at a relatively earlier age. The slowing down process of the thickening could also be the result of the natural "plateau" effect of the growth [10] in thickness of the choroid, in analogy to that of the body size. In other words, it appears that the thickness of the choroid increases in early childhood, reaching a peak between 10 and 20, and then exhibits a gradual decrease into older adulthood $[8,10]$. However, it has to be noted that these assumptions are based on cross-sectional studies and, thus, the same subjects have not been observed regularly during longer periods of time. Therefore, before it can be generalized that the thickness of the choroid increases from early childhood to adolescence, further longitudinal research is ongoing, in which the subjects are being measured regularly and more frequently. 


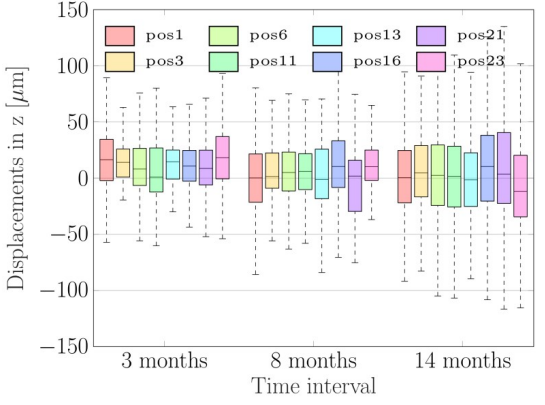

(a)

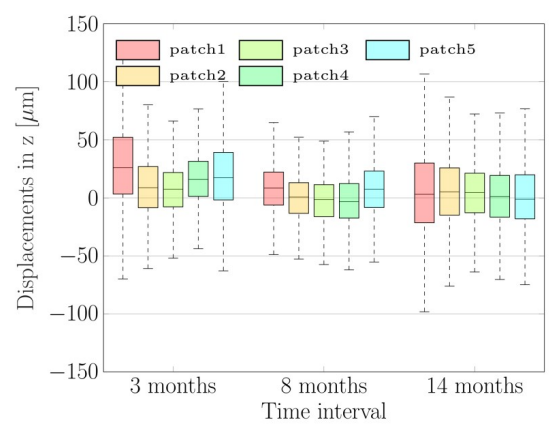

(c)

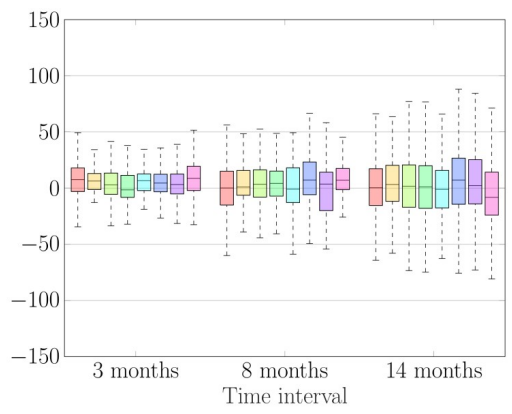

(b)

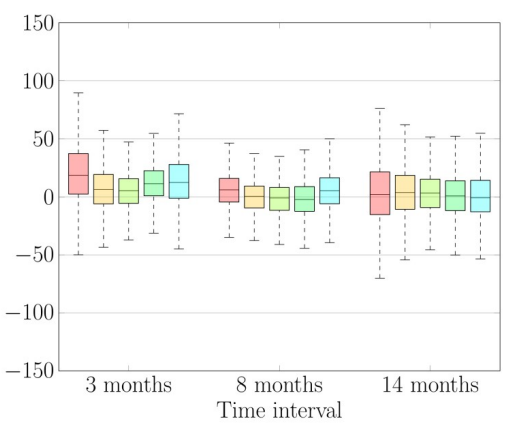

(d)

Fig 9. The average displacements of the CSI grouped by time intervals. Above: The average displacements of the CSI detected by the expert group (a) and by CRAR (b) grouped by time intervals between the two measurements and subdivided into eight scan positions. Below: The average displacements of the CSI detected by the expert group (c) and by CRAR (d) grouped by time interval and subdivided in nasal-temporal/ $x$-direction into five equidistant regions C-B-A-B-C (patches) symmetrically distributed around the foveal center, see Fig 6.

https://doi.org/10.1371/journal.pone.0218776.g009

The time interval related increase in scattering of the detected displacements is a natural consequence of the diversity in choroidal changes, which, most likely, vary from subject to subject.

As mentioned above the results of CRAR are in line with those of other clinical studies: according to $[9,10]$ changes in the choroidal thickness appear to rapidly increase in early childhood (age 4-7, mean increase of $30 \pm 15 \mu \mathrm{m}$ within a time interval of 18 months), followed by a plateau in thickness change in the older age groups examined (age 10-13, mean change $13 \pm 22 \mu \mathrm{m}$ in 18 months). These aspects are reflected in our results and can be explained by the fact that the subjects that took part in this study are in an age phase, in which the choroid is still growing, but not as intensively as in early childhood (4-6 years old).

Fig 9 (c) and 9 (d) show the detected temporal changes grouped by time intervals and subdivided in nasal-temporal $/ x$-direction into five equidistant regions C-B-A-B-C (patches) symmetrically distributed around the foveal center: A (foveal region), B (parafoveal region), and C (perifoveal region), see Fig 6. Here the mean changes detected by CRAR in the regions C-BA-B-C (from the temporal $(\mathrm{T})$ to the nasal $(\mathrm{N})$ location in the case of a right eye, as shown in Fig 6, and in the opposite direction in the case of a left eye): $6.75 \pm 27.12 \mu \mathrm{m}(\mathrm{C}), 4.07 \pm$ $19.23 \mu \mathrm{m}$ (B), $1.21 \pm 13.57 \mu \mathrm{m}$ (A), $4.23 \pm 17.01 \mu \mathrm{m}$ (B) and $5.18 \pm 20.38 \mu \mathrm{m}$ (C), respectively. These values are in the range of those detected by the experts: $9.95 \pm 37.18 \mu \mathrm{m}(\mathrm{C})$, 
6. Statistical Framework for Validation without Ground Truth

PLOS ONE

Statistical framework for validation without ground truth of choroidal thickness changes detection

Table 1. The averaged vertical displacements $\Delta z$ measured at different time intervals. mean $\Delta z$ in $[\mu \mathrm{m}]$ grouped by time interval

\begin{tabular}{l|l|l|l}
\hline mean $\Delta z$ in $[\mu \mathrm{m}]$ grouped by time interval & 3 months & $\mathbf{8}$ months & $\mathbf{1 4}$ months \\
\hline Experts & $1.76 \pm 26.72$ & $5.67 \pm 32.48$ & $7.62 \pm 39.15$ \\
\hline CRAR & $1.41 \pm 16.23$ & $4.88 \pm 22.63$ & $6.12 \pm 29.32$ \\
\hline
\end{tabular}

The table notes the detected temporal changes depicted in Fig 9 (a) and 9 (b) averaged per time interval between the two measurements.

https://doi.org/10.1371/journal.pone.0218776.t001

Table 2. The vertical displacements $\Delta z$ averaged per choroidal subregion.

\begin{tabular}{l|l|l|l|l|l}
\hline \multicolumn{7}{c}{$\Delta \boldsymbol{z}$ in $[\mu \mathrm{m}]$ averaged per choroidal subregion C-B-A-B-C } \\
\hline & C(perifoveal) & B (parafoveal) & A (foveal) & B (parafoveal) & C(perifoveal) \\
\hline Experts & $9.95 \pm 37.18$ & $5.13 \pm 28.53$ & $1.81 \pm 16.21$ & $4.17 \pm 22.37$ & $6.27 \pm 26.01$ \\
\hline CRAR & $6.75 \pm 27.12$ & $4.07 \pm 19.23$ & $1.21 \pm 13.57$ & $4.23 \pm 17.01$ & $5.18 \pm 20.38$ \\
\hline
\end{tabular}

The table notes the detected temporal changes depicted in Fig 9(c) and 9(d) averaged per choroidal subregion C-B-A-B-C (i.e. the five equidistant patches symmetrically distributed around the foveal center).

https://doi.org/10.1371/journal.pone.0218776.t002

$5.13 \pm 28.53 \mu \mathrm{m}(\mathrm{B}), 1.81 \pm 16.21 \mu \mathrm{m}(\mathrm{A}), 4.17 \pm 22.37 \mu \mathrm{m}(\mathrm{B})$ and $6.27 \pm 26.01 \mu \mathrm{m}(\mathrm{C})$, respectively. These results are also summarized in Table 2 for better data visualization.

According to $[32,33]$, the results show a prominent choroidal thickening in more peripheral regions and, in general, it can also be concluded that the first changes occur in the periphery rather than in the center. The error of CRAR is significantly lower than for the remaining five experts after leaving out one expert segmentation $\mathcal{E}_{j}$ at a time $(p$-value $<0.01)$ with medium effect size (Cohen's $d$ in the range of 0.41 and 0.49 ). This emphasizes the superior performance of CRAR in detecting choroidal thickness changes in comparison to those of the experts group.

The power analysis for different time intervals showed a small but not irrelevant effect size, and thus a change in the thickness of the choroid, which cannot be neglected. This supports the tendency mentioned above. For example, comparing the results for the time intervals of 3 and 14 months, we obtained a Cohen's $d$ of 0.20 for the experts and 0.25 for the algorithm.

\section{Conclusion}

In this paper, we presented a statistical framework for validation of choroidal thickness changes detection without ground truth. Using the William's Index, we evaluated the agreement of experts' segmentations and illustrated, with CRAR as example, how to assess their performance in absence of a ground truth for comparison. With the help of the developed framework we demonstrated that our method CRAR provides results which are in the range of those of the experts, but with a lower variability. In addition, we confirmed this outcome using a modified state-of-the-art evaluation procedure: based on the results of a paired t-test, we could attest a higher precision of CRAR for detecting minute thickness changes of the choroid.

It can be concluded that CRAR provides a consistent, automated, expert-level performance in recognizing and monitoring subtle choroidal changes before a disease can actually manifest itself. Thus, we want to further develop CRAR so that it can be applied in the prevention and observation of several diseases and their respective treatments. In the ongoing research we plan to add children between 4-6 years to our test group and to verify the algorithm's performance for a time span of three years, in order to gather more information about the influence 
of age, height, refractive error and axial length on the thickness of the choroid and to exhaustively discuss the results based on our new framework.

All things considered, the proposed validation framework is suitable for analyzing automatic detection algorithms for choroidal thickness changes, but might also be used for other applications, where a ground truth is not available.

\section{Supporting information}

S1 Appendix. Background information about CRAR.

S2 Appendix. Hardware: Hydra-Spectralis.

S1 Table. Choroidal thickness changes measurements. (ZIP)

\section{Acknowledgments}

We would like to express our great appreciation to the HuCE-optoLab's staff that were involved in building the two commercial class prototype OCT systems, as well as their maintenance at Bern University of Applied Sciences, especially Markus Stoller, Michael Peyer, David Luggen, Patrick Arnold, Matthias Mooser and Christian Burri. Furthermore, we are grateful for the commitment of the staff at the Hong Kong Polytechnic University's School of Optometry, who performed the challenging long term study with children and teenagers.

We are grateful to the participating ophthalmologists Dr. Filippo Simona, Dr. Mali Okada, Dr. Daniel Barthelmes, Dr. Simon Rothenbühler and Dr. Emanuel Ramos de Carvalho for their assistance in the manual segmentations; their contributions to our research have been very valuable. We would like to thank the team at our commercial partner Heidelberg Engineering, Ralf Kessler and Tilman Otto for supporting the changes to the Spectralis hardware.

\section{Author Contributions}

Conceptualization: Tiziano Ronchetti, Christoph Jud, Hendrik P. N. Scholl, Boris Považay, Philippe C. Cattin.

Data curation: Tiziano Ronchetti, Christoph Meier, Rachel Ka Man Chun, Quan Liu, Chi-Ho To, Boris Považay.

Formal analysis: Tiziano Ronchetti, Christoph Jud, Peter M. Maloca, Selim Orgül, Alina T. Giger, Rachel Ka Man Chun, Chi-Ho To, Boris Považay, Philippe C. Cattin.

Funding acquisition: Christoph Meier, Boris Považay.

Investigation: Tiziano Ronchetti, Christoph Meier, Rachel Ka Man Chun, Quan Liu, Chi-Ho To, Boris Považay.

Methodology: Tiziano Ronchetti, Christoph Jud, Peter M. Maloca, Alina T. Giger, Hendrik P. N. Scholl, Chi-Ho To, Boris Považay, Philippe C. Cattin.

Project administration: Christoph Meier, Chi-Ho To, Boris Považay.

Resources: Christoph Meier, Boris Považay.

Software: Tiziano Ronchetti. 
Supervision: Christoph Jud, Boris Považay, Philippe C. Cattin.

Validation: Tiziano Ronchetti, Christoph Jud, Peter M. Maloca, Philippe C. Cattin.

Visualization: Tiziano Ronchetti, Peter M. Maloca, Selim Orgül, Rachel Ka Man Chun, Quan Liu.

Writing - original draft: Tiziano Ronchetti, Christoph Jud, Boris Považay, Philippe C. Cattin.

Writing - review \& editing: Tiziano Ronchetti, Christoph Jud, Peter M. Maloca, Selim Orgül, Alina T. Giger, Hendrik P. N. Scholl, Rachel Ka Man Chun, Boris Považay, Philippe C.

Cattin.

\section{References}

1. Nickla DL, Wallman J. The multifunctional choroid. Progress in retinal and eye research. 2010; 29 (2):144-168. https://doi.org/10.1016/j.preteyeres.2009.12.002 PMID: 20044062

2. Chhablani J, Wong IY, Kozak I. Choroidal imaging: A review. Saudi Journal of Ophthalmology. 2014; 28 (2):123-128. https://doi.org/10.1016/j.sjopt.2014.03.004 PMID: 24843305

3. Chakraborty R, Read SA, Collins MJ. Diurnal variations in axial length, choroidal thickness, intraocular pressure, and ocular biometrics. Investigative ophthalmology \& visual science. 2011; 52(8):5121-5129. https://doi.org/10.1167/iovs.11-7364

4. Ikuno $Y$, Kawaguchi $K$, Nouchi T, Yasuno $Y$. Choroidal thickness in healthy Japanese subjects. Investigative ophthalmology \& visual science. 2010; 51(4):2173-2176. https://doi.org/10.1167/iovs.09-4383

5. Park KA, Oh SY. Choroidal thickness in healthy children. Retina. 2013; 33(9):1971-1976. https://doi. org/10.1097/IAE.0b013e3182923477 PMID: 23644561

6. Kim M, Kim SS, Koh HJ, Lee SC. Choroidal thickness, age, and refractive error in healthy Korean subjects. Optometry and Vision Science. 2014; 91(5):491-496. https://doi.org/10.1097/OPX. 0000000000000229 PMID: 24727822

7. Mapelli C, Dell'Arti L, Barteselli G, Osnaghi S, Tabacchi E, Clerici M, et al. Choroidal volume variations during childhood. Investigative ophthalmology \& visual science. 2013; 54(10):6841-6845. https://doi. org/10.1167/iovs.13-12761

8. Read SA, Collins MJ, Vincent SJ, Alonso-Caneiro D. Choroidal Thickness in Childhood. Investigative ophthalmology \& visual science. 2013; 54(5):3586-3593. https://doi.org/10.1167/iovs.13-11732

9. Bidaut-Garnier M, Schwartz C, Puyraveau M, Montard M, Delbosc B, Saleh M. Choroidal thickness measurement in children using optical coherence tomography. Retina. 2014; 34(4):768-774. https:// doi.org/10.1097/IAE.0b013e3182a487a4 PMID: 24013259

10. Read SA, Alonso-Caneiro D, Vincent SJ, Collins MJ. Longitudinal changes in choroidal thickness and eye growth in childhood. Investigative ophthalmology \& visual science. 2015; 56(5):3103-3112. https:// doi.org/10.1167/iovs.15-16446

11. He X, Jin P, Zou H, Li Q, Jin J, Lu L, et al. Choroidal thickness in healthy Chinese children aged 6 to 12 : The Shanghai Children Eye Study. Retina. 2017; 37(2):368-375. https://doi.org/10.1097/IAE. 0000000000001168 PMID: 27429378

12. Wang $D$, Chun RKM, Liu M, Lee RPK, Sun $Y$, Zhang $T$, et al. Optical defocus rapidly changes choroidal thickness in schoolchildren. PloS one. 2016; 11(8):e0161535. https://doi.org/10.1371/journal.pone. 0161535 PMID: 27537606

13. Summers JA. The choroid as a sclera growth regulator. Experimental eye research. $2013 ; 114: 120-$ 127. https://doi.org/10.1016/j.exer.2013.03.008 PMID: 23528534

14. Vuong VS, Moisseiev E, Cunefare D, Farsiu S, Moshiri A, Yiu G. Repeatability of choroidal thickness measurements on enhanced depth imaging optical coherence tomography using different posterior boundaries. American journal of ophthalmology. 2016; 169:104-112. https://doi.org/10.1016/j.ajo.2016 06.023 PMID: 27345731

15. Ho M, Liu DT, Chan VC, Lam DS. Choroidal thickness measurement in myopic eyes by enhanced depth optical coherence tomography. Ophthalmology. 2013; 120(9):1909-1914. https://doi.org/10. 1016/j.ophtha.2013.02.005 PMID: 23683921

16. Považay B, Hermann B, Unterhuber A, Hofer B, et al. Three-dimensional optical coherence tomography at $1050 \mathrm{~nm}$ versus $800 \mathrm{~nm}$ in retinal pathologies: enhanced performance and choroidal penetration in cataract patients. Journal of biomedical optics. 2007; 12(4):041211-041211. https://doi.org/10.1117/1. 2773728 PMID: 17867800 
17. Williams GW. Comparing the joint agreement of several raters with another rater. Biometrics. 1976; $p$. 619-627. https://doi.org/10.2307/2529750 PMID: 963175

18. Ronchetti T, Maloca P, Jud C, Meier C, Orgül S, Scholl HP, et al. Detecting Early Choroidal Changes Using Piecewise Rigid Image Registration and Eye-Shape Adherent Regularization. In: Fetal, Infant and Ophthalmic Medical Image Analysis. Springer; 2017. p. 92-100.

19. Kajić V, Esmaeelpour M, Považay B, Marshall D, Rosin PL, Drexler W. Automated choroidal segmentation of $1060 \mathrm{~nm}$ OCT in healthy and pathologic eyes using a statistical model. Biomedical optics express. 2012; 3(1):86-103. https://doi.org/10.1364/BOE.3.000086 PMID: 22254171

20. Tian J, Marziliano P, Baskaran M, Tun TA, Aung T. Automatic segmentation of the choroid in enhanced depth imaging optical coherence tomography images. Biomedical optics express. 2013; 4(3):397-411. https://doi.org/10.1364/BOE.4.000397 PMID: 23504041

21. Chiu SJ, Li XT, Nicholas P, Toth CA, Izatt JA, Farsiu S. Automatic segmentation of seven retinal layers in SDOCT images congruent with expert manual segmentation. Optics express. 2010; 18(18):1941319428. https://doi.org/10.1364/OE.18.019413 PMID: 20940837

22. Beaton L, Mazzaferri J, Lalonde F, Hidalgo-Aguirre M, Descovich D, Lesk M, et al. Non-invasive measurement of choroidal volume change and ocular rigidity through automated segmentation of highspeed OCT imaging. Biomedical optics express. 2015; 6(5):1694-1706. https://doi.org/10.1364/BOE.6. 001694 PMID: 26137373

23. Chen $\mathrm{Q}$, Fan W, Niu S, Shi J, Shen $\mathrm{H}$, Yuan S. Automated choroid segmentation based on gradual intensity distance in HD-OCT images. Optics express. 2015; 23(7):8974-8994. https://doi.org/10.1364/ OE.23.008974 PMID: 25968734

24. Sui X, Zheng $Y$, Wei B, Bi H, Wu J, Pan X, et al. Choroid segmentation from optical coherence tomography with graph-edge weights learned from deep convolutional neural networks. Neurocomputing. 2017; 237:332-341. https://doi.org/10.1016/j.neucom.2017.01.023

25. SchlegI T, Seeböck P, Waldstein SM, Schmidt-Erfurth U, Langs G. Unsupervised anomaly detection with generative adversarial networks to guide marker discovery. In: International Conference on Information Processing in Medical Imaging. Springer; 2017. p. 146-157.

26. Grau V, Mewes A, Alcaniz M, Kikinis R, Warfield SK. Improved watershed transform for medical image segmentation using prior information. IEEE transactions on medical imaging. 2004; 23(4):447-458. https://doi.org/10.1109/TMI.2004.824224 PMID: 15084070

27. Rex DE, Shattuck DW, Woods RP, Narr KL, Luders E, Rehm K, et al. A meta-algorithm for brain extraction in MRI. Neurolmage. 2004; 23(2):625-637. https://doi.org/10.1016/j.neuroimage.2004.06.019 PMID: 15488412

28. Zijdenbos AP, Forghani R, Evans AC. Automatic "pipeline" analysis of $3-D \mathrm{MRI}$ data for clinical trials: application to multiple sclerosis. IEEE transactions on medical imaging. 2002; 21(10):1280-1291. https://doi.org/10.1109/TMI.2002.806283 PMID: 12585710

29. Martin-Fernandez M, Bouix S, Ungar L, McCarley RW, Shenton ME. Two methods for validating brain tissue classifiers. In: International Conference on Medical Image Computing and Computer-Assisted Intervention. Springer; 2005. p. 515-522.

30. Kim HS, Park SB, Lo SS, Monroe JI, Sohn JW. Bidirectional local distance measure for comparing segmentations. Medical physics. 2012; 39(11):6779-6790. https://doi.org/10.1118/1.4754802 PMID: 23127072

31. Sullivan GM, Feinn R. Using effect size - or why the $P$ value is not enough. Journal of graduate medica education. 2012; 4(3):279-282. https://doi.org/10.4300/JGME-D-12-00156.1 PMID: 23997866

32. Park KA, Oh SY. Analysis of spectral-domain optical coherence tomography in preterm children: retinal layer thickness and choroidal thickness profiles. Investigative ophthalmology \& visual science. 2012; 53 (11):7201-7207. https://doi.org/10.1167/iovs.12-10599

33. Ruiz-Moreno JM, Flores-Moreno I, Lugo F, Ruiz-Medrano J, Montero JA, Akiba M. Macular choroidal thickness in normal pediatric population measured by swept-source optical coherence tomography. Investigative ophthalmology \& visual science. 2013; 54(1):353-359. https://doi.org/10.1167/iovs.1210863 


\subsection{Further Experiments}

In order to attest the superior performance of CRAR in recognizing thickness changes, we applied CRAR to the 90 OCT volume stack pairs after artificially induced deformation [167]. The mean errors in detecting changes by CRAR were compared to those obtained applying the old version of the algorithm, a state-of-the-art segmentation method based on graph search [173] and manual expert segmentation. Figure 6.1 illustrates that the higher precision of CRAR is remarkable, in particular at a higher resolution level. As a reminder, we point out that a multiresolution approach for the registration was used in CRAR (for more detail see [28]). In this context, $k$ denotes the resolution level with the corresponding number of patches in which the volume surrounding the CSI is subdivided in nasal-temporal $/ x$-direction, i.e. $8,16, \ldots, 512$ for $k=1,2, \ldots, 7$. In Fig. 3.3 the situation for $k=1$ and 8 blocks is shown.

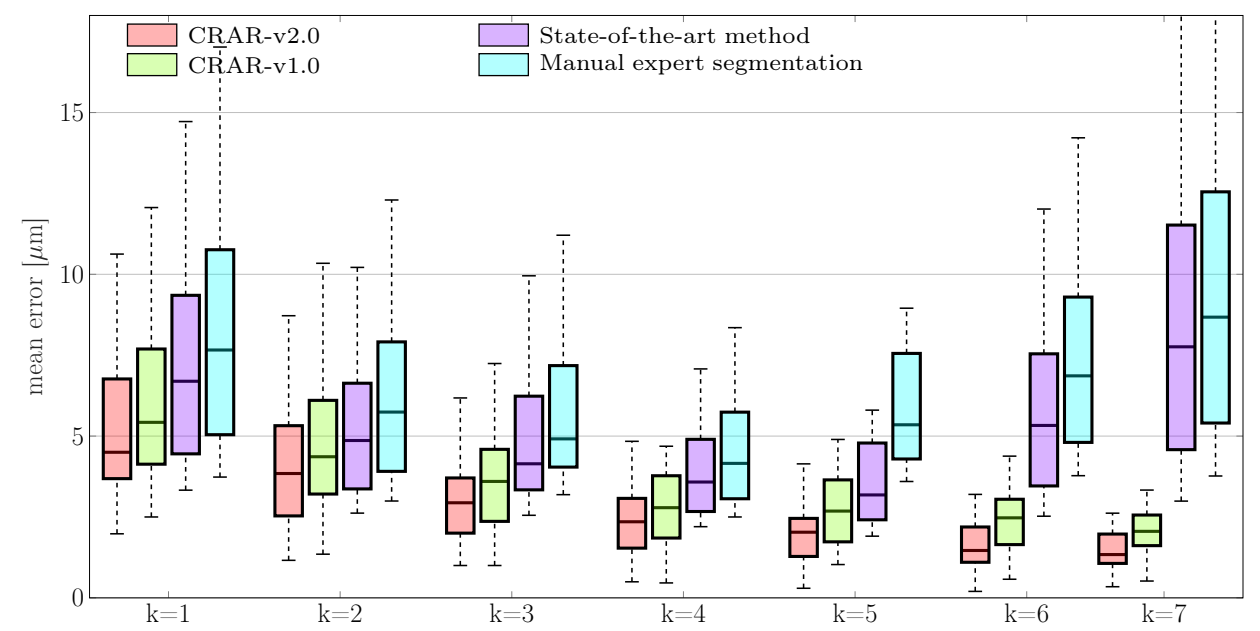

Figure 6.1.: Validation of CRAR.

The average differences between the synthetically induced displacements and the measured ones, obtained with CRAR-v2.0 (red), CRAR-v1.0 (green), a state-ofthe-art graph search based segmentation method [173] (purple) and manual expert segmentation (cyan), applied to the 90 OCT volume stack pairs after synthetic deformation for different resolution levels $k$.

\subsection{Supplementary Information}

For the interested reader we add two short excursions into the choice of the metric and the adapted power analysis used in the developed statistical framework.

Choice of Metric

As mentioned in the publication, the algorithm of our choice uses a registration-based approach and provides the displacement field of the surrounding region of the CSI in the vertical $/ z$-direction as a result, without having to look for the exact position of the CSI. Therefore, as illustrated in Fig. 7, the calculation of Williams' index (WI), comparing algorithm and expert group, uses only the value for the metric diffZ, but there is no information regarding the values of Dice/Jaccard 
and BLD, because the results are neither areas nor contours. Of course, our framework is also suitable for (segmentation-based) algorithms, where the outcomes are contours (segmentation lines).

\section{Adapted Power Analysis}

With the help of a minimal example implemented in Matlab (see Fig. 6.2), we want to sweep away any remaining concerns on the side of the reader about the necessity of the power analysis presented in the paper. It is true that our "leave-one-out" power analysis does not have a direct influence on the calculation of the $p$-values, because this calculation is based on the difference between the inputs of the paired t-test. Thus, the $p$-values of $X-\overline{\mathcal{G}}_{j}$ vs. $\Delta \mathcal{E}_{j}-\overline{\mathcal{G}}_{j}$ and $X$ vs. $\Delta \mathcal{E}_{j}$ are the same. In other words, the results of the paired t-test do not change, if both inputs are compared with the same reference, here $\overline{\mathcal{G}}_{j}$. However, such a comparison has an influence on the effect size. This is because in our case, $\overline{\mathcal{G}}_{j}$ has to be represented as a vector, as for every OCT B-scan pair a corresponding ground truth for comparison has to be generated.

As illustrated in Fig. 6.2, we calculated the $p$-values and Cohen's $d$ for the cases $X$ vs. $Y:=\Delta \mathcal{E}_{j}$ and $X_{j}:=X-\overline{\mathcal{G}}_{j}$ vs. $Y_{j}:=\Delta \mathcal{E}_{j}-\overline{\mathcal{G}}_{j}$, where $G_{j}:=\overline{\mathcal{G}}_{j}$ denotes the common reference (or ground truth for comparison). In the case of $X$ vs. $Y$, positive and negative values can be interpreted as "thickening" or "thinning" of the CSI. If $X_{j}$ vs. $Y_{j}$ is considered, this means the algorithm or the expert has over- or underestimated the value of the temporal changes in the thickness of the CSI. The workspace (see Fig. 6.2(b)) shows that both the calculation of the $p$-values and Cohen's $d$ for $X$ vs. $Y$ and $X_{j}$ vs. $Y_{j}$ give the same results, if $G_{j}$ is a scalar.

However, if $G_{j}$ is a vector, only the $p$-values remain the same, while the values of Cohen's $d$ for $X_{j}$ vs. $Y_{j}$ are different from those for $X$ vs. $Y$. In this case, the values of Cohen's $d$, and therefore the effect size, are influenced by the changes in the standards deviations (see the values of "std" in the workspace). In conclusion, because in our case the ground truth can only be represented in the form of a vector, the use of such a "leave-one-out" power analysis is justified. 


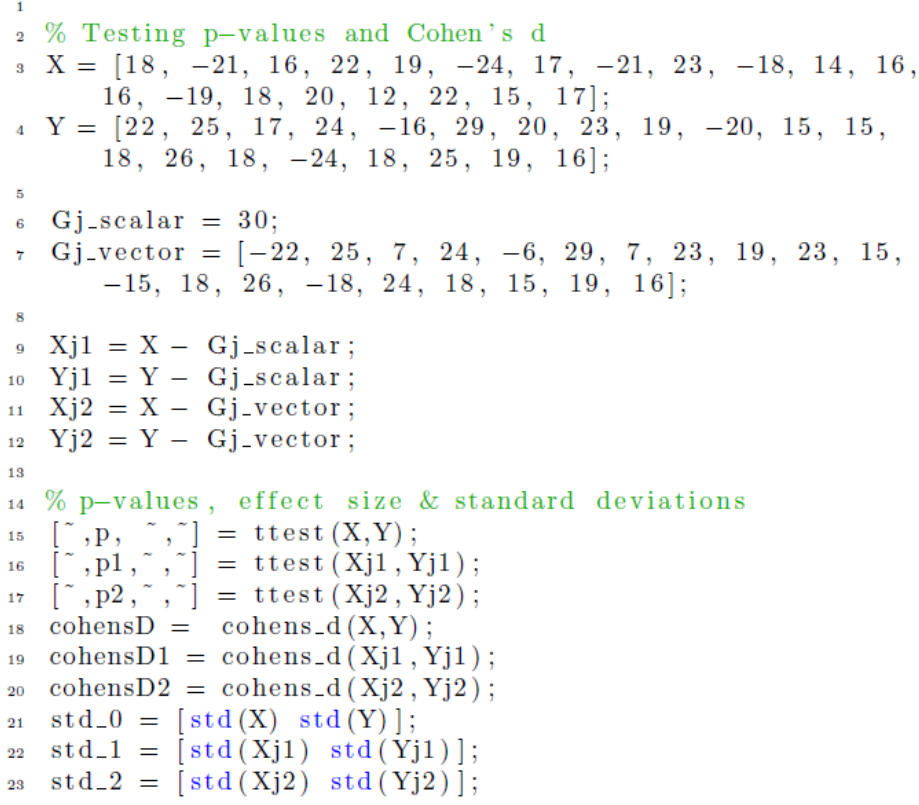

(a)

\begin{tabular}{|c|c|c|}
\hline Workspace & & (?) \\
\hline Name- & Value & \\
\hline$\boxplus$ cohensD & -0.3886 & \\
\hline (1) cohensD1 & -0.3886 & \\
\hline 田 cohensD2 & -0.2544 & \\
\hline 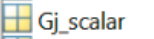 & 30 & \\
\hline 画Gjvector & $1 \times 20$ double & \\
\hline 田p & 0.2598 & \\
\hline 画1 & 0.2598 & \\
\hline 田p2 & 0.2598 & \\
\hline std_0 & {$[17.2410,15.3913]$} & \\
\hline 田std_1 & {$[17.2410,15.3913]$} & \\
\hline 画std_2 & [28.1086,21.3564] & \\
\hline$\boxplus x$ & $1 \times 20$ double & \\
\hline$\boxplus \mathrm{x}_{\mathrm{j} 1}$ & $1 \times 20$ double & \\
\hline Xj2 & $1 \times 20$ double & \\
\hline 畐Y & $1 \times 20$ double & \\
\hline 到1 & $1 \times 20$ double & \\
\hline 画 $\mathrm{Yj}_{2}$ & $1 \times 20$ double & \\
\hline
\end{tabular}

(b)

Figure 6.2.: Minimal example implemented in Matlab.

(a) Code, and (b) workspace with the inputs and outputs of a minimal example implemented in Matlab, to show that our "leave-one-out" power analysis does not have a direct influence on the calculation of the $p$-values. In other words, the results of the paired t-test do not change, if both inputs are compared with the same reference, here $\overline{\mathcal{G}}_{j}$. However, such a comparison has an influence on the effect size. This is because in our case, $\overline{\mathcal{G}}_{j}$ has to be represented as a vector. 


\title{
7. Feasibility Study of Subfoveal Choroidal Thickness Changes in Spectral-Domain Optical Coherence Tomography Measurements of Macular Telangiectasia Type 2
}

\begin{abstract}
Macular telangiectasia type 2 (MacTel2) is an ocular disease which manifests itself without noticeable symptoms in its early stages. Later, due to similar patterns of neovascularization, these symptoms could be mistaken for age-related macular degeneration (AMD) [82]. As the disease progresses, blurring, distorted vision, and a loss of central vision can occur within a period of 10-20 years. MacTel2 does not affect side vision, nor does it usually lead to total blindness. All existing knowledge about this disease is restricted to its advanced stages, while its beginnings and early stages are still unclear [81]. This publication describes a first feasibility study aiming to find out if choroidal thickness changes are correlated with the ongoing development of MacTel2. ${ }^{1}$
\end{abstract}

\section{Publication}

This conference paper was published in the book Computational Pathology and Ophthalmic Medical Image Analysis, Springer, Cham, 2018, pp. 303-309, [80]. It was presented at the Fifth International Workshop for Ophthalmic Medical Image Analysis (OMIA5), MICCAI 2018, Granada, Spain. $^{2}$

\footnotetext{
${ }^{1}$ It has to be noted, that the statistical validation framework, presented in Chapter 6 , was not used for the evaluation of the results presented in this publication. This is due to the fact this was published before the validation framework was completed.

${ }^{2}$ This paper is available at Springer Professional: https://www.springerprofessional.de/feasibility-study-ofsubfoveal-choroidal-thickness-changes-in-sp/16122544.
} 


\title{
Feasibility Study of Subfoveal Choroidal Thickness Changes in Spectral-Domain Optical Coherence Tomography Measurements of Macular Telangiectasia Type 2
}

Tiziano Ronchetti ${ }^{1,2,3(\bowtie)}$, Peter Maloca ${ }^{2,4,6}$, Emanuel Ramos de Carvalho ${ }^{6}$, Tjebo F. C. Heeren ${ }^{5}$, Konstantinos Balaskas ${ }^{5,6}$, Adnan Tufail ${ }^{6}$, Catherine Egan ${ }^{6}$, Mali Okada ${ }^{6}$, Selim Orgül $^{4}$, Christoph Jud ${ }^{1}$, and Philippe C. Cattin ${ }^{1}$

1 Center for medical Image Analysis and Navigation (CIAN),

Department of Biomedical Engineering, University of Basel, Allschwil, Switzerland Tiziano.Ronchetti@unibas.ch

2 OCTlab, Department of Ophthalmology, University of Basel, Basel, Switzerland

${ }^{3}$ HuCE-optoLab, Bern University of Applied Sciences, Biel, Switzerland

${ }^{4}$ University Hospital Basel, Basel, Switzerland

5 Moorfields Ophthalmic Reading Centre, London, UK

${ }^{6}$ Moorfields Eye Hospital, London, UK

\begin{abstract}
Macular Telangiectasia Type $2(\mathrm{MacTel} 2)$ is a disease of the retina leading to a gradual deterioration of central vision. At the onset of the disease a good visual acuity is present, which declines as the disease progresses to cause reading difficulties. In this paper, we present new insights on the vascular changes in MacTel2. We investigated whether MacTel2 progression correlates to changes in the thickness of the choroid. For this purpose, we apply a recently published registration-based approach to detect deviations in the choroid on a dataset of $45 \mathrm{MacTel} 2$ patients. Between 2012 and 2016 these subjects and a control group were measured twice within variable intervals of time in the Moorfields Eye Hospital in the MacTel Natural History Observation and Registry Study. Our results show that in the MacTel2 group the thickness of the choroid increased while in the control group a decrease was noted. Manual expert segmentation and an automated state-of-the-art method were used to validate the results.
\end{abstract}

Keywords: Macular Telangiectasia Type 2

Choroidal thickness changes $\cdot$ Piecewise rigid registration

\section{Introduction}

Macular Telangiectasia Type 2 (MacTel2) [3] is a disease of the macula, causing loss of central vision. Typically, in MacTel2, perifoveal vessels leak and/or

(C) Springer Nature Switzerland AG 2018

D. Stoyanov et al. (Eds.): COMPAY 2018/OMIA 2018, LNCS 11039, pp. 303-309, 2018. 
become dilated. Fluid from leakage causes macular swelling [17], which can decrease visual acuity.

MacTel2 can be present without noticeable symptoms in the early stages. As the disease progresses, blurring, distorted vision, and loss of central vision can be detected and lead to a loss of central vision over a period of 10 to 20 years. MacTel2 does not affect side vision, nor does it usually lead to total blindness. Due to the lack of early symptoms and an adequate therapy for MacTel2, it is important to obtain early and regular eye exams. It may be an inherited disease and, especially in its later stage, it could be mistaken for age-related macular degeneration (AMD) [6], due to similar patterns of neovascularization. But all existing knowledge is restricted to advanced stages of the disease, while its beginnings and early stages are still unclear [3].

MacTel2 is diagnosed with the help of optical coherence tomography (OCT) [12] and specified in detail using fluorescein angiography (FA) [3]. There is no therapy of MacTel2. However, in the case of neovascular vessel growth in the course of MacTel2, the injection of anti-vascular growth agents has been shown to be effective.

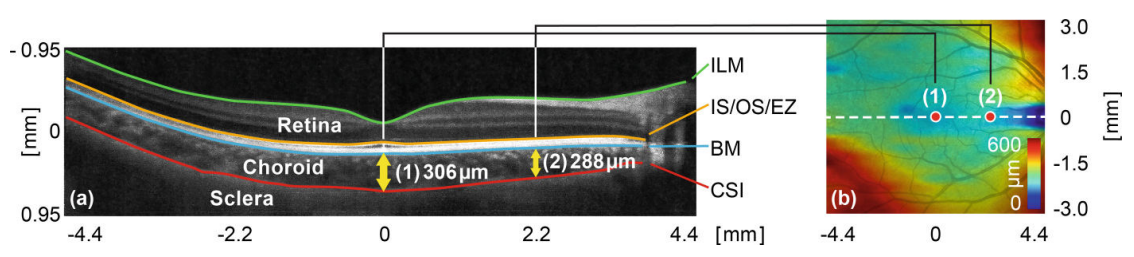

Fig. 1. (a) OCT B-scan with segmented layers corresponding to the white dotted line in (b). Inner Limiting Membrane (ILM), Bruch's Membrane (BM) and Choroid-Sclera Interface (CSI). IS/OS/EZ denotes the Inner Segment/Outer Segment/Ellipsoid Zone. (b) Choroidal (BM-CSI) thickness map overlaid color-coded on fundus image. The indicated locations correspond to the central (1) and paracentral (2) choroidal thickness measurements, respectively.

Choroidal thickness (ChT) has been demonstrated to be an important biomarker for MacTel2 $[2,7,9]$ and other pathologies such as age-related macular degeneration (AMD), diabetic macular edema (DME) [17], glaucoma [8] and juvenile myopia [14]. Therefore, monitoring of the choroidal thickness delivers insight into the pathogenesis of such diseases and helps in the planning of their treatment. With this feasibility study, we want to evaluate the impact of the choroidal thickness over time in MacTel2.

\section{State-of-the-Art Research}

The authors of [11] examined microcystoid spaces in MacTel2 patients. Evidence was found in the pathogenesis of MacTel2 because the Müller cells dysfunction was reported. Not much research has been done to correlate choroidal thickness changes in the course of MacTel2. The authors of [1] examined choroidal thickness changes in eyes and concluded that the thickness of the choroid did not 
vary between the eyes of MacTel2 patients and those of age-matched healthy subjects. In contrast, the authors of $[10,15]$ compared subfoveal choroidal thickness in a similarly large group of MacTel2 patients to that of healthy subjects. They concluded that patients with MacTel2 show a clearly thicker choroid in the subfoveal area.

In this paper, we examine if a correlation between changes in the thickness of the choroid and the development of MacTel 2 exists. We validate our results with the help of a state-of-the-art method [5] and manual segmentation by two experts.

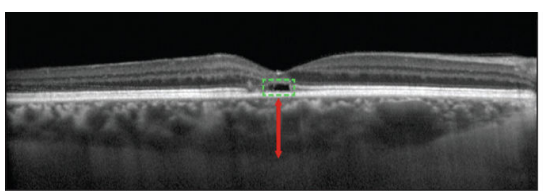

(a) Subfoveal ChT in MacTel2 (arrow)

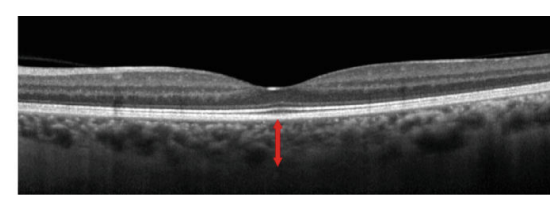

(b) Subfoveal ChT in a healthy eye (arrow)

Fig. 2. (a) Right eye of a 53-year-old woman with MacTel2. The horizontal B-scan shows the increased subfoveal choroidal thickness of $422 \mu \mathrm{m}$ (arrow). Typical findings of MacTel2 include an intraretinal outer retinal cavity and loss of the IS/OS/EZ indicated with the dashed box. (b) Healthy right eye of a 53-year-old woman from the control group.

\section{Material and Method}

The basis of this retrospective study was formed by forty-five MacTel2 patients who were enrolled at Moorfields Eye Hospital in the MacTel Natural History Observation and Registry Study from June 2012 until March 2016.

Their mean age was 57.1 (range 42 to 71 ). The study was approved by the local institutional review board and conducted according to the tenets of the Declaration of Helsinki. The volunteers were divided into three groups: the first group was measured a second time after 1 year, the second group after 2 years, the third after 4 years. Both eyes were measured.

Optical coherence tomography was acquired using the Heidelberg Spectralis, Human Reliability Analysis 2 (HRA2) system (Heidelberg Engineering, Heidelberg, Germany). The scan pattern for MacTel2 patients was between $3.8 \times 2.5 \times 1.9 \mathrm{~mm}^{3}$ and $4.4 \times 2.9 \times 1.9 \mathrm{~mm}^{3}$ including $49-261$ B-scans per volume, imaging averaging $8-12$ scans, interslice distance was $11-30 \mu \mathrm{m}$. The scan pattern for all healthy subjects was defined by raster lines of $4.5 \times 3.0 \times 1.9 \mathrm{~mm}^{3}$ (261 B-scans, averaging 8-12 scans, interslice distance was $11 \mu \mathrm{m}$ ).

The thickness of the choroid was calculated for each A-scan, defined as the Euclidean distance between the Bruch's Membrane (BM) and the posterior surface of the choroid, delimited by the Choroid-Sclera Interface (CSI, see Fig. 1(a)). 
Choroidal thickness maps for the $6 \times 6 \mathrm{~mm}^{2}$ macula-centered region imaged by spectral-domain (SD)-OCT scans were created (see Fig. 1(b)) and the average thicknesses were reported in micrometers. The changes in the choroidal thickness, measured between two visits, were automatically detected using CRAR [14], a registration-based algorithm, especially developed for longitudinal studies, which tackles the problems that occur in using image segmentation (i.e. low contrast, loss of signal and artifacts), the common approach to localize the exact position of the CSI.

Instead of segmentation, CRAR uses a piecewise rigid image registrationbased approach, in which the changes in thickness in the region around the CSI are examined. In such a way the exact position of the CSI is no longer needed. In CRAR the uniform smoothness of the results is supported by a regularization which matches the anatomic structure of the eye [14].

In the next step, CRAR' s results were compared with those obtained using a graph search-based state-of-the-art segmentation method [5] and manual expert segmentation by two independent experts. Finally, the subfoveal choroidal thickness was determined. Since MacTel2 manifests itself in the juxtafoveal region, it is reasonable to assume that the subfoveal choroidal thickness is reliable for this kind of analysis (see Fig. 2(a)).

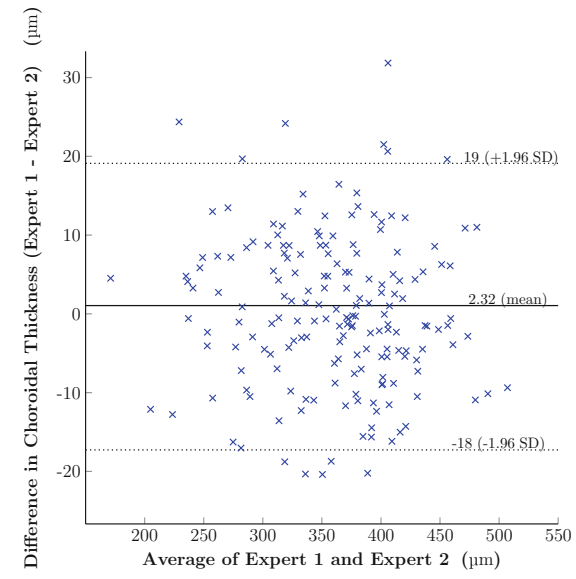

(a) Intraobserver experts' agreement

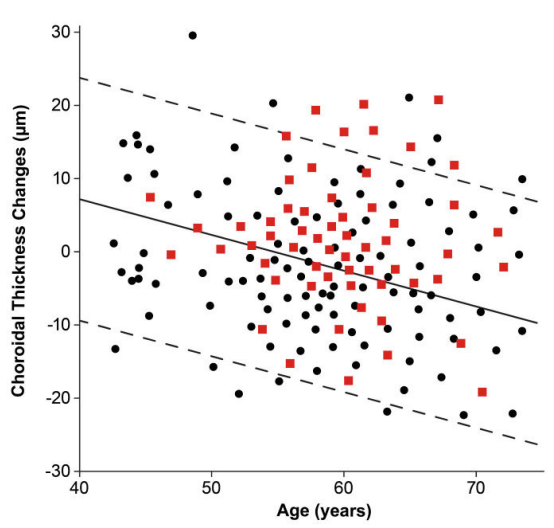

(b) Subfoveal ChT vs age

Fig. 3. (a) Bland-Altman plot representing the agreement between the choroidal thickness measured by the two experts. (b) Scatter plot comparing the relationship of subfoveal choroidal thickness and age in MacTel2 eyes (red squares) vs healthy control eyes (black dots). The dashed lines indicate $95 \%$ normal tolerance limits for choroidal thickness per year. 


\section{Result}

Subfoveal choroidal thickness was manually measured independly by two experts, who showed an agreement with an interobserver correlation $R^{2}$ of 0.96 for both the MacTel2 affected and the healthy eyes. A Bland-Altman diagram in Fig. 3(a) shows a mean interobserver difference of $2.32 \mu \mathrm{m}$, while the $95 \%$ limits of agreement are -18 and $19 \mu \mathrm{m}$, respectively.

There was a predominance of females among both the MacTel2 subjects (32 $\widehat{=} 71 \%)$ and healthy subjects $(28 \widehat{=} 62 \%)$. However, there was no difference in the results between the groups with respect to gender $(P=0.068$, Fisher' $\mathrm{s}$ exact test). Mean ages in the study and control groups were $58.4 \pm 9.3$ ( $\widehat{=} 49-68$ years) and $52 \pm 15.8$ ( $\widehat{=} 36-68$ years), respectively.

The relationship between changes in the subfoveal choroidal thickness and age in the MacTel2 and control group are shown in Fig. 3(b). All changes in the subfoveal choroidal thickness of both the MacTel2 and control group subdivided into time intervals between the two measurements are illustrated in Fig. 4(a).

On average, the subfoveal choroidal thickness of the MacTel2 patients increased in the time interval between the two measurements with $2.47 \pm 7.89 \mu \mathrm{m}$ (after 1 year), $4.32 \pm 13.12 \mu \mathrm{m}$ ( 2 years) and $10.85 \pm 21.43$ (4 years). In the case of healthy eyes, the same measurements showed a decrease of the subfoveal choroidal thickness of $-0.71 \pm 6.35 \mu \mathrm{m},-3.92 \pm 10.76 \mu \mathrm{m},-8.37 \pm 15.01 \mu \mathrm{m}$, respectively. The total mean increase of the MacTel2 group (over all time intervals and subjects) was $5.88 \pm 14.15 \mu \mathrm{m}$, while in case of the control group this decrease was $-4.33 \pm 10.71 \mu \mathrm{m}$ (see Fig. $4(\mathrm{~b})$ ). On average, the subfoveal choroidal thickness decreased by $2.55 \mu \mathrm{m}$ per year.

There is a decrease in the subfoveal choroidal thickness in adults ranging from $14-54 \mu \mathrm{m}$ every ten years $[4,16]$. On the other hand, the thickness increased in the MacTel2 group by $2.79 \mu \mathrm{m}$ per year. The increase of the MacTel2 slope showed no correlation to the decrease of the control group slope, nor the other way round $(P=0.54$, test of interaction).

For the whole MacTel 2 group, the state-of-the-art algorithm provided mean subfoveal choroidal thickness changes of $5.02 \pm 10.55 \mu \mathrm{m}$, while both experts in average $6.84 \pm 21.14 \mu \mathrm{m}$. For the whole control group the detected subfoveal choroidal thickness changes were $-4.81 \pm 12.87 \mu \mathrm{m}$ and $-6.32 \pm 19.14 \mu \mathrm{m}$. After conducting a power analysis, we could attest a superior performance of CRAR vs the state-of-the-art-method ( $P<0.05$, medium effect size as Cohen's $d$ in the range $0.43-0.51$, paired t-test), as well as vs the manual expert segmentation $(P<0.001$, large effect size as Cohen's distance $d>0.8$, paired t-test).

\section{Discussion}

As mentioned above, our main findings is the increase of the choroidal thickness in MacTel2 patients compared to healthy subjects. Similar results were mentioned in $[10,15]$, where authors also came to the conclusion that a statistically significant positive correlation was found exist between subfoveal choroidal thickness and age in MacTel2 subjects, while subfoveal choroidal thickness and age 
are negatively correlated in healthy adults $[4,16]$. A thickening of the choroid may be an early manifestation of MacTel2 eyes and therefore be potentially useful for diagnosis and monitoring. In previous studies of MacTel2 eyes, there has been no mention of an abnormal choroid [13]. However, diurnal changes were not considered in this study what can be a limiting factor. Müller cell dysfunction might be behind the pathogenesis of MacTel2, because Müller' s cell loss is commonly associated with macular pigment depletion [13]. Müller cell dysfunction may potentially lead to changes not only in the retinal but also in the choroidal vessels.

Based on our findings of choroidal involvement, the thesis that MacTel2 is only linked to the retina needs to be reconsidered as there is a reasonable assumption that the choroid may also be involved. Another limitation regarding this study is that we only measured the subfoveal choroidal thickness, whereas the rest of the choroid was not evaluated. But because the disease activity is primarily present in the juxtafoveal region, it is nevertheless reasonable to assume that the subfoveal choroidal thickness is reliable for this kind of analysis.

Finally, the age disparity between patients with MacTel2 and healthy subjects should be taken more into consideration in future studies, although even when adjusted for age, using analysis of covariance as shown in [10], the differences in choroidal thickness measurements between MacTel2 and control eyes persisted.

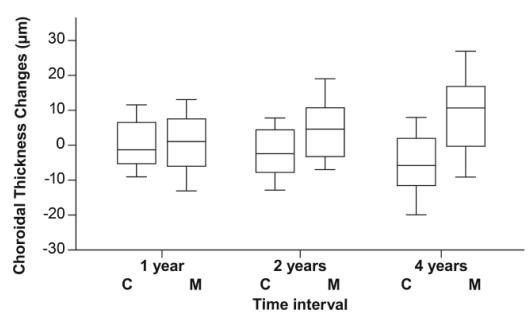

(a) Subfoveal ChT per time interval

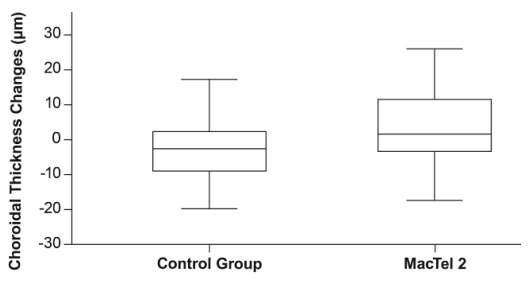

(b) Total subfoveal ChT

Fig. 4. (a) Subfoveal ChT changes detected by CRAR in the control (C) and MacTel2 (M) group subdivided into the different time intervals between the two measurements. (b) Total average of subfoveal ChT changes for MacTel2 and control group.

\section{Conclusion and Outlook}

A thickened choroid may be a valuable diagnostic and monitoring clue in identifying MacTel2, and this may lead to better understand its pathogenesis. Further studies are needed to validate the prognostic value of these findings and to determine whether there are quantitative or qualitative changes in the choroid that predict the onset and progression of this disease. 


\section{References}

1. Chhablani, J., et al.: Choroidal thickness in macular telangiectasia type 2. Retina 34(9), 1819-1823 (2014)

2. Chhablani, J., Wong, I.Y., Kozak, I.: Choroidal imaging: a review. Saudi J. Ophthalmol. 28(2), 123-128 (2014)

3. Issa, P.C., et al.: Macular telangiectasia type 2. Prog. Retin. Eye Res. 34, 49-77 (2013)

4. Margolis, R., Spaide, R.F.: A pilot study of enhanced depth imaging optical coherence tomography of the choroid in normal eyes. Am. J. Ophthalmol. 147(5), 811815 (2009)

5. Mazzaferri, J., Beaton, L., Hounye, G., Sayah, D.N., Costantino, S.: Open-source algorithm for automatic choroid segmentation of oct volume reconstructions. Sci. Rep. 7, 42112 (2017)

6. McLeod, D.S., Grebe, R., Bhutto, I., Merges, C., Baba, T., Lutty, G.A.: Relationship between RPE and choriocapillaris in age-related macular degeneration. Investig. Ophthalmol. Vis. Sci. 50(10), 4982-4991 (2009)

7. Mutti, D.O., Gwiazda, J., Norton, T.T., Smith III, E.L., Schaeffel, F., To, C.: Myopia-yesterday, today, and tomorrow. Optom. Vis. Sci. 90(11), 1161 (2013). Offcial publication of the American Academy of Optometry

8. Mwanza, J.C., Hochberg, J.T., Banitt, M.R., Feuer, W.J., Budenz, D.L.: Lack of association between glaucoma and macular choroidal thickness measured with enhanced depth-imaging optical coherence tomography. Investig. Ophthalmol. Vis. Sci. 52(6), 3430-3435 (2011)

9. Nickla, D.L., Wallman, J.: The multifunctional choroid. Prog. Retin. Eye Res. 29(2), 144-168 (2010)

10. Nunes, R.P., et al.: Spectral-domain optical coherence tomography measurements of choroidalthickness and outer retinal disruption in macular telangiectasia type 2 . Ophthalmic Surg. Lasers Imaging Retin. 46(2), 162-170 (2015)

11. Okada, M., Egan, C.A., Heeren, T.F., Tufail, A., Fruttiger, M., Maloca, P.M.: Macular telangiectasia type 2: quantitative analysis of a novel phenotype and implications for the pathobiology of the disease. Retina 38, S97-S104 (2018)

12. Považay, B., Hermann, B., Unterhuber, A., Hofer, B.: Three-dimensional optical coherence tomography at $1050 \mathrm{~nm}$ versus $800 \mathrm{~nm}$ in retinal pathologies: enhanced performance and choroidal penetration in cataract patients. J. Biomed. Opt. 12(4), 041211 (2007)

13. Powner, M.B., Gillies, M.C., Zhu, M., Vevis, K., Hunyor, A.P., Fruttiger, M.: Loss of müller's cells and photoreceptors in macular telangiectasia type 2. Ophthalmology 120(11), 2344-2352 (2013)

14. Ronchetti, T., et al.: Detecting early choroidal changes using piecewise rigid image registration and eye-shape adherent regularization. In: Cardoso, M.J., et al. (eds.) FIFI/OMIA -2017. LNCS, vol. 10554, pp. 92-100. Springer, Cham (2017). https:// doi.org/10.1007/978-3-319-67561-9_10

15. Shah, M., et al.: Subfoveal choroidal thicknessin macular telangiectasia type 2. Investig. Ophthalmol. Vis. Sci. 53(14), 2138 (2012)

16. Wei, W.B., et al.: Subfoveal choroidal thickness: the Beijing eye study. Ophthalmology 120(1), 175-180 (2013)

17. Yiu, G., Manjunath, V., Chiu, S.J., Farsiu, S., Mahmoud, T.H.: Effect of antivascular endothelial growth factor therapy on choroidal thickness in diabetic macular edema. Am. J. Ophthalmol. 158(4), 745-751 (2014) 


\section{Discussion and Conclusion}

In this chapter, we summarize the results achieved, as well as the strengths and limitations of the developed method. Furthermore, we take a look at future research.

\subsection{Achievements}

The goal of this work was to develop a method to automatically detect choroidal thickness changes in longitudinal studies. In particular, the method should be able to overcome the problem of low SNR in OCT images at the border area separating the choroid from the adjacent sclera.

In the orientation phase of the study current state-of-the-art methods, which are almost all segmentation-based were tested, on the problem at hand. It became clear very quickly, that these approaches suffer strongly from the high variability of the signal during the acquisition of the images in the lower choroidal region, close to the sclera. For this reason, we opted for image registration, instead of image segmentation, as the main tool to develop our method.

The underlying idea consisted of analyzing the changes in the area surrounding the CSI instead of insisting on localizing its exact position. This way, a rough positioning of the CSI, which we obtained by using the rigid BM as a reference surface, sufficed. We subdivided the volume around the CSI into partially overlapping cuboidal subregions. After we had aligned the images to the rigid BM, the displacement corresponded to shifts of the CSI. As a result, we obtained the displacement field around the CSI and used its outcome to quantify the choroidal growth.

Because of the interleaved nature of the eye's tunics, which consist of the soft choroid and its surrounding more rigid tissues, the sclera and retina, we opted for a piecewise rigid strategy [162] to model the deformation of the choroid. CRAR takes this interleaved nature of the eye into account and allows decomposing the global non-rigid matching problem of the choroid into numerous local rigid registrations of the individual subregions. The fine subdivision into blocks allows to investigate the deformations of the choroid by a global non-rigid transformation model, although the displacements of the individual subregions are rigidly modeled. The results were embodied into a dense global non-rigid deformation field built in such a way that it elastically deformed the soft choroid and preserved the rigid characteristics of the surrounding sclera and retina.

This method was created in a three-step process.

1. In the first version (see Chapter 4), we showed the feasibility of our detection method on a simplified model by creating a prototype for detecting temporal choroidal thickness changes, using a block matching-based image registration approach. Testing this prototype on scanrescan data provided encouraging results, showing robustness regarding noise. However, further improvements were necessary, because this first draft operated only scan-wise in 2D. Another limitation was that the regularization was implemented only as a post-processing step. As a matter of fact, the mismatched blocks were corrected only after the registration, 


\section{Discussion and Conclusion}

using a constraint that minimized the curvature of the connecting line through the block centers toward a fixed minimal value. Moreover, in this process the pixel spacing was not taken into account. Finally, the use of non-overlapping patches in the matching process led to a deterioration of the results with increasing resolution level, due to poor information in the fine block width.

2. In the next step (see Chapter 5), we extended the registration framework into 3D and designed an eye-shape adherent regularization system, which operates parallel to the 3D matching process. As a main difference from the original idea of the previous 2D approach, we subdivided the volume around the CSI into partially overlapping subregions, utilizing $\mathrm{BM}$ as a reference surface to determine the displacement field in the axial $(z-)$ direction of the choroid. In order to achieve a more natural representation of the choroidal deformation, we opted for the radial cubic B-spline, due to its smoothing properties and finite support. We thus obtained the following achievements: we extended the method from $2 \mathrm{D}$ to $3 \mathrm{D}$ by grouping several rectangular subregions around the CSI into cuboidal blocks. The regularization penalizes radial differences of the transformations and favors similar displacements of patches within the same neighborhood, which mimics the mechanical behavior of the rigid scleral tunic [166]. Moreover, we regularized the detected displacements (along the $z$-axis) with respect of the $x$ - and $y$-coordinates of all block centers within a neighborhood (corresponding to the support of the kernel used). The results showed a uniform smoothness which matches the anatomic structure of the eye. In a pre-clinical experiment, we first tested the method's precision using scan-rescan data. Furthermore, by introducing smooth synthetic deformations of the rescans (up to $50 \mu \mathrm{m}$ ), we proved a superior change-detection performance of our method in comparison to manual expert segmentation.

3. The precision in the detection of choroidal thickness changes was achieved in the range of the pixel scaling in the $z$-direction. Even for finer block sizes, changes smaller than $2 \mu \mathrm{m}$ could be reliably recognized, allowing subvoxel accuracy. By grouping the slices, the amount of information per patch increased, thereby reducing mismatches.

4. Finally (see Chapter 6), we presented an efficient statistical validation framework to evaluate its (and other methods') detection-performance in case a real ground truth is not available. Besides, this framework might also be used for other applications, where an algorithm for automated detection has to be validated, but a ground truth for comparison is missing.

In order to investigate further application fields for our method, we conducted a retrospective study in collaboration with the Moorfields Eye Hospital in London. We analyzed the eyes of adults affected with MacTel2 and already found interesting results (see Chapter 7). They let us assume that a correlation may exist between changes in the thickness of the choroid and the development of MacTel2. At least they suggest that the choroidal thickness might increase for adults with MacTel2, in contrast to the decrease that commonly takes place around the age of 50. As in the case of juvenile myopia, we obtained results for MacTel2 that are in line with those of other studies. This fact makes us feel confident to have developed a method with considerable potential for clinical applications. 


\subsection{Critical Points/Limitations}

The method presented was specifically developed for longitudinal studies, since it was designed to detect temporal changes in thickness, without depending on any particular definition of CSI. In other words, the goal of CRAR is not to determine the exact position of the CSI, but to determine the thickness changes obtained from OCT data acquired from the same subject at different time points. Using CRAR we can determine the displacement field of the CSI but not the exact location of the CSI itself.

Therefore, the method's strength is at the same time its inherent weakness: CRAR is not applicable in cross-sectional studies that investigate differences between individuals at a single time point. However, CRAR shows superior subvoxel accuracy in long-time study data. Another inherent limitation is that the accuracy of the results strongly depends on the rigid pre-registration step consisting of laterally aligning at BM two or more images of the same subject taken at different points in time. We evaluated the displacement field on the axial direction ( $z$-axis) only, and not on the lateral direction ( $x$ - and $y$-axis), because we merely focused on juvenile myopia, which is mainly related to changes in thickness and not to structural alterations of the choroid. The expansion or contraction, growth and/or drastic changes in the texture of tissue were explicitly not part of the current model. This is because these are not common in the investigated diseases either, and, therefore, do not require such a sensitive means of quantification.

Finally, the main purpose of this method is to detect changes in the thickness of the choroid as soon as possible. At this point, the choroid has not yet altered its shape very much and the natural uniform smoothness of the eye is still present, so a natural eye-shape adherent regularization model is ideal. As soon as the disease is in an advanced stage, the use of such a model is no longer suitable.

\subsection{Outlook}

In future research work, we aim to analyze the influence of age on the changes in the thickness of the choroid. The results of several studies $[16,18]$ indicate that the most significant changes happen at the age between 4 and 6 . Since most of the children we have evaluated so far were between 8 and 13 years of age, it makes sense to include younger children in future studies.

Therefore, the setup of the study will have to be redesigned: in the ongoing longitudinal research we plan to add children between 4-6 years to our test group and to verify the algorithm's performance for longer periods of time. The same children should be examined within a measurement period of a total of 2-3 years at regular 3-month intervals in order to gather more information about the influence of age, height, refractive error and axial length on the thickness of the choroid and to exhaustively discuss the results based on our validation framework.

As mentioned earlier, CRAR has been developed with the purpose to detect minute temporal changes in the choroidal thickness. Of course, it can also be applied to further examine changes in the intra-retinal layers, see Sec. 2.1.4. However, the quality of OCT images in this area is significantly better than in the lower region of the choroid, and the limit for quantification is rather given by physiological effects during the measurement itself (e.g. pulsation or blood/ocular pressure variation). Therefore, for such tasks, standard segmentation methods already provide a satisfactory solution. It would, however, be interesting to compare their results with those 


\section{Discussion and Conclusion}

of CRAR to investigate if this extremely sensitive algorithm is able to unveil more accurate information.

As a next step, in the ongoing myopia research, we plan on expanding CRAR to detect changes in the $x$ - and $y$-direction. Monitoring the lateral development of the choroidal tissue could lead to a better understanding of ocular diseases other than myopia which are also characterized by alterations in the structure of the choroid rather than by changes in its thickness.

We also see great potential in expanding CRAR in other medical imaging fields (e.g. OCTdermatology, ultrasound imaging), especially where low signal to noise and reproducible environments can be found, which makes a reliable segmentation of tissues very challenging, and where minute changes in tissues have to be monitored as potentially disease-related.

\subsection{Conclusion}

The development of OCT devices is continuous and fast, which means that in future it might be possible to obtain OCT images at such a high resolution to allow successful, direct segmentation of the CSI. However, as to the current state-of-the-art methods (and because of varying signal quality in different subjects and increasing acquisition speed), we still believe that finding the CSI using image segmentation is not the best approach. The common segmentation algorithms are often implemented in such a way that they are influenced by human expectation: human experts have a specific idea what the CSI is supposed to look like in areas with lower resolution and poor contrast. The constraints are often defined accordingly. The basic problem remains that a ground truth to assess the reliability of the segmentation is not available. The only tool at our available are the manual expert segmentations which obviously vary from expert to expert. Even when a consensus on segmenting has been reached, repeated segmentations of the same image, done by the same expert, will always differ from each other.

Graph-based segmentations can fail because of the rather poor image contrast, while the new emerging deep learning methods produce very good results, particularly when they replace a human expert in segmentation tasks. However, deep learning methods are still influenced by the preconceived perception of the experts. Moreover, the results of such methods are harder to interpret and can be influenced by subtle textures that are not obvious to the human investigators. In order to overcome this problem we would need a huge amount of training data, which would obviously be very expensive and difficult to realize. CRAR is more objective because it is based on algorithmic calculations only (so no training data) and thus free from any human bias in determining a ground truth.

It can be concluded that CRAR provides a consistent, automated, expert-level performance with subvoxel accuracy in recognizing and monitoring subtle choroidal changes before an eye disease actually manifests itself. Thus, we want to further develop CRAR so that it can be applied in the prevention and observation of several diseases and their respective treatments. A registration-based approach for temporal thickness changes detection was developed, which allows to overcome the problems in the segmentation of OCT images. At its present stage of development, CRAR enables unsupervised, automated detection and quantification of choroidal changes and can extract minute differences in large, paired data sets. 


\section{Bibliography}

[1] O. Braddick and J. Atkinson, "Development of human visual function," Vision research, vol. 51, no. 13, pp. 1588-1609, 2011.

[2] J. D. Wright Jr and W. P. Boger III, "Visual complaints from healthy children," Survey of ophthalmology, vol. 44, no. 2, pp. 113-121, 1999.

[3] D. Turbert, "Normal vision development in children," https://www.aao.org/eye-health/ tips-prevention/children-vision-development, 2017.

[4] A. Zimmermann, K. M. M. d. Carvalho, C. Atihe, S. M. V. Zimmermann, and V. L. d. M. Ribeiro, "Visual development in children aged 0 to 6 years," Arquivos brasileiros de oftalmologia, vol. 82, no. 3, pp. 173-175, 2019.

[5] M. Sundy and A. K. Lauer, "Pathologic myopia (myopic degeneration)," https://eyewiki. aao.org/Pathologic_myopia_(myopic_degeneration), 2016.

[6] A. M. Miller, A. Barash, A. Murchison, B. H. Feldman, P. O. Phelps, P. A. Karth, and S. J. Patel, "Retinal detachment," https://eyewiki.org/Retinal_Detachment, 2019.

[7] R. F. Spaide, K. Ohno-Matsui, L. A. Yannuzzi et al., Pathologic myopia. Springer, 2014.

[8] K. Ohno-Matsui, "Pathologic myopia," The Asia-Pacific Journal of Ophthalmology, vol. 5, no. 6, pp. 415-423, 2016.

[9] Y. Zhou, M. Song, M. Zhou, Y. Liu, F. Wang, and X. Sun, "Choroidal and retinal thickness of highly myopic eyes with early stage of myopic chorioretinopathy: tessellation," Journal of ophthalmology, vol. 2018, 2018.

[10] Y. Ikuno, S. Fujimoto, Y. Jo, T. Asai, and K. Nishida, "Choroidal thinning in high myopia measured by optical coherence tomography," Clinical ophthalmology (Auckland, NZ), vol. 7, p. $889,2013$.

[11] I. G. Morgan, K. Ohno-Matsui, and S.-M. Saw, "Myopia," The Lancet, vol. 379, no. 9827, pp. 1739-1748, 2012.

[12] B. Gilmartin, "Myopia: precedents for research in the twenty-first century," Clinical \& experimental ophthalmology, vol. 32, no. 3, pp. 305-324, 2004.

[13] M. Ho, D. T. Liu, V. C. Chan, and D. S. Lam, "Choroidal thickness measurement in myopic eyes by enhanced depth optical coherence tomography," Ophthalmology, vol. 120, no. 9, pp. 1909-1914, 2013.

[14] S. Mrejen and R. F. Spaide, "Optical coherence tomography: imaging of the choroid and beyond," Survey of ophthalmology, vol. 58, no. 5, pp. 387-429, 2013. 


\section{Bibliography}

[15] S. Wang, Y. Wang, X. Gao, N. Qian, and Y. Zhuo, "Choroidal thickness and high myopia: a cross-sectional study and meta-analysis," BMC ophthalmology, vol. 15, no. 1, p. 70, 2015.

[16] S. A. Read, M. J. Collins, S. J. Vincent, and D. Alonso-Caneiro, "Choroidal thickness in childhood," Investigative ophthalmology \& visual science, vol. 54, no. 5, pp. 3586-3593, 2013.

[17] S. A. Read, D. Alonso-Caneiro, S. J. Vincent, and M. J. Collins, "Longitudinal changes in choroidal thickness and eye growth in childhood," Investigative ophthalmology \& visual science, vol. 56, no. 5, pp. 3103-3112, 2015.

[18] M. Bidaut-Garnier, C. Schwartz, M. Puyraveau, M. Montard, B. Delbosc, and M. Saleh, "Choroidal thickness measurement in children using optical coherence tomography," Retina, vol. 34, no. 4, pp. 768-774, 2014.

[19] Y. Ikuno, K. Kawaguchi, T. Nouchi, and Y. Yasuno, "Choroidal thickness in healthy japanese subjects," Investigative ophthalmology \& visual science, vol. 51, no. 4, pp. 2173$2176,2010$.

[20] K.-A. Park and S. Y. Oh, "Choroidal thickness in healthy children," Retina, vol. 33, no. 9, pp. 1971-1976, 2013.

[21] M. Kim, S. S. Kim, H. J. Koh, and S. C. Lee, "Choroidal thickness, age, and refractive error in healthy korean subjects," Optometry and Vision Science, vol. 91, no. 5, pp. 491496, 2014.

[22] C. Mapelli, L. Dell'Arti, G. Barteselli, S. Osnaghi, E. Tabacchi, M. Clerici, R. Ratiglia, and F. Viola, "Choroidal volume variations during childhood," Investigative ophthalmology \& visual science, vol. 54, no. 10, pp. 6841-6845, 2013.

[23] X. He, P. Jin, H. Zou, Q. Li, J. Jin, L. Lu, H. Zhao, J. He, X. Xu, M. Wang et al., "Choroidal thickness in healthy chinese children aged 6 to 12: The shanghai children eye study," Retina, vol. 37, no. 2, pp. 368-375, 2017.

[24] D. Wang, R. K. M. Chun, M. Liu, R. P. K. Lee, Y. Sun, T. Zhang, C. Lam, Q. Liu, and C. H. To, "Optical defocus rapidly changes choroidal thickness in schoolchildren," PloS one, vol. 11, no. 8, p. e0161535, 2016.

[25] J. A. Summers, "The choroid as a sclera growth regulator," Experimental eye research, vol. 114, pp. 120-127, 2013.

[26] D. L. Nickla and J. Wallman, "The multifunctional choroid," Progress in retinal and eye research, vol. 29, no. 2, pp. 144-168, 2010.

[27] V. S. Vuong, E. Moisseiev, D. Cunefare, S. Farsiu, A. Moshiri, and G. Yiu, "Repeatability of choroidal thickness measurements on enhanced depth imaging optical coherence tomography using different posterior boundaries," American journal of ophthalmology, vol. 169, pp. 104-112, 2016. 
[28] T. Ronchetti, P. Maloca, C. Jud, C. Meier, S. Orgül, H. P. Scholl, B. Považay, and P. C. Cattin, "Detecting early choroidal changes using piecewise rigid image registration and eye-shape adherent regularization," in Fetal, Infant and Ophthalmic Medical Image Analysis. Springer, 2017, pp. 92-100.

[29] J. Chhablani, I. Y. Wong, and I. Kozak, "Choroidal imaging: A review," Saudi Journal of Ophthalmology, vol. 28, no. 2, pp. 123-128, 2014.

[30] D. O. Mutti, J. Gwiazda, T. T. Norton, E. L. Smith III, F. Schaeffel, and C.-h. To, "Myopia-yesterday, today, and tomorrow," Optometry and vision science: official publication of the American Academy of Optometry, vol. 90, no. 11, p. 1161, 2013.

[31] E. H. Myrowitz, "Juvenile myopia progression, risk factors and interventions," Saudi Journal of Ophthalmology, vol. 26, no. 3, pp. 293-297, 2012.

[32] S. S. Rong, L. J. Chen, and C. P. Pang, "Myopia Genetics-The Asia-Pacific Perspective," The Asia-Pacific Journal of Ophthalmology, vol. 5, no. 4, pp. 236-244, 2016.

[33] T. Ronchetti, P. Maloca, C. Meier, S. Orgül, C. Jud, P. Hasler, B. Považay, and P. C. Cattin, "Intensity-based choroidal registration using regularized block matching," in Proceedings of the Ophthalmic Medical Image Analysis Third International Workshop (OMIA), 2016, pp. 33-40.

[34] H. F. Krey, G. Grunau, and H. Bräuer, Exempla ophthalmologica: Bildatlas zur Physiologie und Pathophysiologie des Auges. Albert-Roussel-Pharma, 1986.

[35] R. Machemer, G. Michelson, and G. Naumann, "Atlas of ophthalmology," https://www. atlasophthalmology.net/.

[36] B. Feldman, "Eyewiki," https://eyewiki.aao.org/Main_Page.

[37] Wikipedia, "Wikipedia," https://www.wikipedia.org/.

[38] "International statistical classification of diseases and related health problems 11th revision," http://www.who.int/classifications/icd/revision/en/.

[39] Vision Eye Institute, "How the eye works," https://visioneyeinstitute.com.au/eyematters/ how-the-eye-works/.

[40] Wikipedia, "Visual cortex," https://en.wikipedia.org/wiki/Visual_cortex.

[41] R. S. Ramrattan, T. L. van der Schaft, C. M. Mooy, W. De Bruijn, P. Mulder, and P. De Jong, "Morphometric analysis of bruch's membrane, the choriocapillaris, and the choroid in aging." Investigative ophthalmology \& visual science, vol. 35, no. 6, pp. 2857$2864,1994$.

[42] P. Lin, P. S. Mettu, D. L. Pomerleau, S. J. Chiu, R. Maldonado, S. Stinnett, C. A. Toth, S. Farsiu, and P. Mruthyunjaya, "Image inversion spectral-domain optical coherence tomography optimizes choroidal thickness and detail through improved contrast," Investigative ophthalmology \& visual science, vol. 53, no. 4, pp. 1874-1882, 2012. 
9. Bibliography

[43] L. M. Parver, "Temperature modulating action of choroidal blood flow," Eye, vol. 5, no. 2, p. 181, 1991.

[44] N. Carpenter and A. P. Griorian, "Hyperopia," https://eyewiki.aao.org/Hyperopia, 2015.

[45] L. Ostrow, Gregory I. and Kirkeby, "Myopia," https://eyewiki.aao.org/Myopia, 2019.

[46] Vision Web, "Nearsightedness," https://www.visionweb.com/content/aboutvw/ aboutcontent.jsp?RID $=27$.

[47] The Eye Institute for Medicine \& Surgery, "Myopia (nearsightedness)," http://www. seebetterbrevard.com/disorders/myopia.php.

[48] B. Rajan, "High myopia treatment," http://high-myopia-eye-hospital.com/low-myopia/.

[49] S. Gunasekaran, T. Agarwal, N. Sharma, and R. Tandon, "Refractive surgery for myopia: Case selection and management options," National Medical Journal of India, vol. 27, no. 4, pp. 204-209, 2014.

[50] J. Hjortdal, "Surface ablation techniques for myopia-a review of the advances over the past 25 years," European Ophthalmic Review, vol. 11, no. 1, pp. 31-34, 2017.

[51] C. S. Y. Lam, W. C. Tang, D. Y.-Y. Tse, Y. Y. Tang, and C. H. To, "Defocus incorporated soft contact (disc) lens slows myopia progression in hong kong chinese schoolchildren: a 2-year randomised clinical trial," British Journal of Ophthalmology, vol. 98, no. 1, pp. 40-45, 2014.

[52] J. T. Leung and B. Brown, "Progression of myopia in hong kong chinese schoolchildren is slowed by wearing progressive lenses." Optometry and vision science: official publication of the American Academy of Optometry, vol. 76, no. 6, pp. 346-354, 1999.

[53] J. Gwiazda, L. Hyman, M. Hussein, D. Everett, T. T. Norton, D. Kurtz, M. C. Leske, R. Manny, W. Marsh-Tootle, and M. Scheiman, "A randomized clinical trial of progressive addition lenses versus single vision lenses on the progression of myopia in children," Investigative ophthalmology \& visual science, vol. 44, no. 4, pp. 1492-1500, 2003.

[54] P. Cho, S. W. Cheung, and M. H. Edwards, "Practice of orthokeratology by a group of contact lens practitioners in hong kong-part 1. general overview." Clinical \& experimental optometry, vol. 85, no. 6, pp. 365-371, 2002.

[55] P. Cho, S. W. Cheung, and M. H. Edwards, "Practice of orthokeratology by a group of contact lens practitioners in hong kong: Part 2. orthokeratology lenses," Clinical and Experimental Optometry, vol. 86, no. 1, pp. 42-46, 2003.

[56] G. Lingham, D. A. Mackey, R. Lucas, and S. Yazar, "How does spending time outdoors protect against myopia? A review," British Journal of Ophthalmology, 2019.

[57] D. O. Mutti, L. T. Sinnott, S. A. Cotter, L. A. Jones-Jordan, R. N. Kleinstein, R. E. Manny, J. D. Twelker, and K. Zadnik, "Childhood age, time outdoors, and the risk of juvenile-onset myopia," Investigative Ophthalmology \& Visual Science, vol. 59, no. 9, pp. 3392-3392, 2018. 
[58] M. Chen, A. Wu, L. Zhang, W. Wang, X. Chen, X. Yu, and K. Wang, "The increasing prevalence of myopia and high myopia among high school students in fenghua city, eastern china: a 15-year population-based survey," BMC ophthalmology, vol. 18, no. 1, p. $159,2018$.

[59] C. S. Y. Lam, E. Goldschmidt, and M. H. Edwards, "Prevalence of myopia in local and international schools in hong kong," Optometry and vision science, vol. 81, no. 5, pp. 317-322, 2004.

[60] Y. Li, J. Liu, and P. Qi, "The increasing prevalence of myopia in junior high school students in the haidian district of beijing, china: a 10-year population-based survey," $B M C$ ophthalmology, vol. 17, no. 1, p. 88, 2017.

[61] I. G. Morgan, M. He, and K. A. Rose, "Epidemic of pathologic myopia: what can laboratory studies and epidemiology tell us?" Retina, vol. 37, no. 5, pp. 989-997, 2017.

[62] S. J. Ryan, "Retina. London: Saunders," Elsevier. Retrieved September, vol. 13, p. 2016, 2013.

[63] T. Y. Wong, A. Ferreira, R. Hughes, G. Carter, and P. Mitchell, "Epidemiology and disease burden of pathologic myopia and myopic choroidal neovascularization: an evidencebased systematic review," American journal of ophthalmology, vol. 157, no. 1, pp. 9-25, 2014 .

[64] M. C. Lim, S.-T. Hoh, P. J. Foster, T.-H. Lim, S.-J. Chew, S. K. Seah, and T. Aung, "Use of optical coherence tomography to assess variations in macular retinal thickness in myopia," Investigative Ophthalmology \& Visual Science, vol. 46, no. 3, pp. 974-978, 2005.

[65] B. J. Curtin and D. B. Karlin, "Axial length measurements and fundus changes of the myopic eye," American journal of ophthalmology, vol. 71, no. 1, pp. 42-53, 1971.

[66] M. Takano and S. Kishi, "Foveal retinoschisis and retinal detachment in severely myopic eyes with posterior staphyloma," American journal of ophthalmology, vol. 128, no. 4, pp. 472-476, 1999.

[67] Y. Ikuno, "Overview of the complications of high myopia," Retina, vol. 37, no. 12, pp. 2347-2351, 2017.

[68] Genetics Home Reference, "Gyrate atrophy of the choroid and retina," https://ghr.nlm. nih.gov/condition/gyrate-atrophy-of-the-choroid-and-retina.

[69] Wikipedia, "Retinal detachment," https://en.wikipedia.org/wiki/Retinal_detachment, 2019.

[70] M. P. Passarinho, M. N. Cohen, and A. S. Vinay, "Eyewiki," http://eyewiki.aao.org/ Horseshoe_or_flap_tear.

[71] F. Gillespie and B. Covelli, "Hereditary high myopia with retinal detachment: A family study," Archives of Ophthalmology, vol. 69, no. 6, pp. 733-736, 1963. 
[72] V. Nerschbach, J. Eule, N. Eberle, R. Höinghaus, and D. Betz, "Ocular manifestation of lymphoma in newly diagnosed cats," Veterinary and comparative oncology, vol. 14, no. 1, pp. 58-66, 2016.

[73] NYU Langone Health, "Procedures to treat retinal tears and retinal detachments," https://nyulangone.org/conditions/retinal-detachment/treatments/ procedures-to-treat-retinal-tears-retinal-detachments.

[74] P. A. Asbell, I. Dualan, J. Mindel, D. Brocks, M. Ahmad, and S. Epstein, "Age-related cataract," The Lancet, vol. 365, no. 9459, pp. 599-609, 2005.

[75] P. A. Campochiaro, "Retinal and choroidal neovascularization," Journal of cellular physiology, vol. 184, no. 3, pp. 301-310, 2000.

[76] C. Millsap, G. Peyman, and M. Greve, "Subretinal hemorrhage removal with multiple retinotomy sites in age-related macular degeneration," Ophthalmic Literature, vol. 3, no. 48 , p. $214,1995$.

[77] National Eye Institute (NEI), "Glaucoma," https://www.nei.nih.gov/search?terms= glaucoma\&language $=$ All\&type $=$ All.

[78] P. Mitchell, W. Smith, K. Attebo, and P. R. Healey, "Prevalence of open-angle glaucoma in australia: the blue mountains eye study," Ophthalmology, vol. 103, no. 10, pp. 1661$1669,1996$.

[79] Baxter Eye Care, "Glaucoma testing and treatment," https://www.baxtereye.com/ eye-care-services/management-of-ocular-diseases/glaucoma-testing-and-treatment/.

[80] T. Ronchetti, P. Maloca, E. R. de Carvalho, T. F. Heeren, K. Balaskas, A. Tufail, C. Egan, M. Okada, S. Orgül, C. Jud et al., "Feasibility study of subfoveal choroidal thickness changes in spectral-domain optical coherence tomography measurements of macular telangiectasia type 2," in Computational Pathology and Ophthalmic Medical Image Analysis. Springer, 2018, pp. 303-309.

[81] P. C. Issa, M. C. Gillies, E. Y. Chew, A. C. Bird, T. F. Heeren, T. Peto, F. G. Holz, and H. P. Scholl, "Macular telangiectasia type 2," Progress in retinal and eye research, vol. 34, pp. 49-77, 2013.

[82] D. S. McLeod, R. Grebe, I. Bhutto, C. Merges, T. Baba, and G. A. Lutty, "Relationship between rpe and choriocapillaris in age-related macular degeneration," Investigative ophthalmology \& visual science, vol. 50, no. 10, pp. 4982-4991, 2009.

[83] B. Považay, B. Hermann, A. Unterhuber, B. Hofer et al., "Three-dimensional optical coherence tomography at $1050 \mathrm{~nm}$ versus $800 \mathrm{~nm}$ in retinal pathologies: enhanced performance and choroidal penetration in cataract patients," Journal of biomedical optics, vol. 12, no. 4, pp. 041211-041211, 2007.

[84] G. Chang, C. Westerfeld, A. Lane, and J. Miller, "Intravitreal bevacizumab (avastin) in the treatment of macular telangiectasis type 2 (mactel 2)," Investigative Ophthalmology \& Visual Science, vol. 50, no. 13, pp. 756-756, 2009. 
[85] F. Pichi, K. Aggarwal, P. Neri, P. Salvetti, A. Lembo, P. Nucci, C. M. G. Cheung, and V. Gupta, "Choroidal biomarkers," Indian journal of ophthalmology, vol. 66, no. 12, p. $1716,2018$.

[86] P. Phadikar, S. Saxena, S. Ruia, T. Y. Lai, C. H. Meyer, and D. Eliott, "The potential of spectral domain optical coherence tomography imaging based retinal biomarkers," International journal of retina and vitreous, vol. 3, no. 1, p. 1, 2017.

[87] J. W. Shin, Y. U. Shin, H. Y. Cho, and B. R. Lee, "Measurement of choroidal thickness in normal eyes using 3d oct-1000 spectral domain optical coherence tomography," Korean Journal of Ophthalmology, vol. 26, no. 4, pp. 255-259, 2012.

[88] K. B. Freund, L. A. Yannuzzi, and J. A. Sorenson, "Age-related macular degeneration and choroidal neovascularization," American journal of ophthalmology, vol. 115, no. 6, pp. 786-791, 1993.

[89] G. Yiu, V. Manjunath, S. J. Chiu, S. Farsiu, and T. H. Mahmoud, "Effect of antivascular endothelial growth factor therapy on choroidal thickness in diabetic macular edema," American journal of ophthalmology, vol. 158, no. 4, pp. 745-751, 2014.

[90] J.-C. Mwanza, J. T. Hochberg, M. R. Banitt, W. J. Feuer, and D. L. Budenz, "Lack of association between glaucoma and macular choroidal thickness measured with enhanced depth-imaging optical coherence tomography," Investigative ophthalmology \& visual science, vol. 52, no. 6, pp. 3430-3435, 2011.

[91] Q. Chen, S. Huang, Q. Ma, H. Lin, M. Pan, X. Liu, F. Lu, and M. Shen, "Ultra-high resolution profiles of macular intra-retinal layer thicknesses and associations with visual field defects in primary open angle glaucoma," Scientific reports, vol. 7, p. 41100, 2017.

[92] T. Oberwahrenbrock, M. Weinhold, J. Mikolajczak, H. Zimmermann, F. Paul, I. Beckers, and A. U. Brandt, "Reliability of intra-retinal layer thickness estimates," PloS one, vol. 10, no. 9, p. e0137316, 2015.

[93] G. Staurenghi, S. Sadda, U. Chakravarthy, R. F. Spaide et al., "Proposed lexicon for anatomic landmarks in normal posterior segment spectral-domain optical coherence tomography: the in*oct consensus," Ophthalmology, vol. 121, no. 8, pp. 1572-1578, 2014.

[94] P. Soukup, P. Maloca, B. Altmann, M. Festag, E.-A. Atzpodien, and S. Pot, "Interspecies variation of outer retina and choriocapillaris imaged with optical coherence tomography," Investigative ophthalmology \& visual science, vol. 60, no. 10, pp. 3332-3342, 2019.

[95] C. H. Meyer, S. Saxena, and S. R. Sadda, Spectral domain optical coherence tomography in macular diseases. Springer, 2016.

[96] H. Gray and W. Lewis, "Anatomy of the human body. lea \& febiger, philadelphia," From: Bartleby. com, 1918.

[97] Wikipedia, "Retina," https://en.wikipedia.org/wiki/Retina.

[98] H. Kolb, "Simple anatomy of the retina," The Organization of the Retina and Visual System. Webvision, 2011. 
9. Bibliography

[99] Wikipedia, "Fluorescein angiography," https://en.wikipedia.org/wiki/Fluorescein_ angiography.

[100] R. F. Spaide, J. M. Klancnik, and M. J. Cooney, "Retinal vascular layers imaged by fluorescein angiography and optical coherence tomography angiography," JAMA ophthalmology, vol. 133, no. 1, pp. 45-50, 2015.

[101] I. Kozak, V. L. Morrison, T. M. Clark, D.-U. Bartsch, B. R. Lee, I. Falkenstein, A. M. Tammewar, F. Mojana, and W. R. Freeman, "Discrepancy between fluorescein angiography and optical coherence tomography in detection of macular disease," Retina (Philadelphia, Pa.), vol. 28, no. 4, p. 538, 2008.

[102] J. F. De Boer, R. Leitgeb, and M. Wojtkowski, "Twenty-five years of optical coherence tomography: the paradigm shift in sensitivity and speed provided by fourier domain oct," Biomedical optics express, vol. 8, no. 7, pp. 3248-3280, 2017.

[103] W. Drexler and J. G. Fujimoto, Optical coherence tomography: technology and applications. Springer Science \& Business Media, 2008.

[104] S. Aumann, S. Donner, J. Fischer, and F. Müller, "Optical coherence tomography (oct): Principle and technical realization," in High Resolution Imaging in Microscopy and Ophthalmology. Springer, 2019, pp. 59-85.

[105] A. Kumar, J. I. Lim, N. Bhagat, and Y. Gupta, "Wide field retinal imaging systems," https://eyewiki.aao.org/Wide_field_retinal_imaging_systems.

[106] L. A. Yannuzzi, K. T. Rohrer, L. J. Tindel, R. S. Sobel, M. A. Costanza, W. Shields, and E. Zang, "Fluorescein angiography complication survey," Ophthalmology, vol. 93, no. 5, pp. 611-617, 1986.

[107] M. Bhende, S. Shetty, M. K. Parthasarathy, and S. Ramya, "Optical coherence tomography: A guide to interpretation of common macular diseases," Indian journal of ophthalmology, vol. 66, no. 1, p. 20, 2018.

[108] J. Fingler, C. Readhead, D. M. Schwartz, and S. E. Fraser, "Phase-contrast oct imaging of transverse flows in the mouse retina and choroid," Investigative ophthalmology \& visual science, vol. 49, no. 11, pp. 5055-5059, 2008.

[109] D. Pauleikhoff, B. Heimes, G. Spital, M. Gutfleisch, M. Ziegler, B. Book, M. Farecki, and A. Lommatzsch, "Oct angiography-is this the future for macular diagnosis?" Klinische Monatsblätter für Augenheilkunde, vol. 232, no. 9, pp. 1069-1076, 2015.

[110] E. Mozayan and P. A. Mammo, Danny A. Karth, "Macular telangiectasia," https:// eyewiki.aao.org/Macular_telangiectasia.

[111] A. F. Fercher, G. von Bally, and S. Khanna, "Ophthalmic interferometry optics in medicine, biology and environmental research," 1990.

[112] D. Huang, E. A. Swanson, C. P. Lin, J. S. Schuman, W. G. Stinson, W. Chang, M. R. Hee, T. Flotte, K. Gregory, C. A. Puliafito et al., "Optical coherence tomography," Science, vol. 254, no. 5035, pp. 1178-1181, 1991. 
[113] O. Ferrer-Roca, "Telepathology and optical biopsy," International journal of telemedicine and applications, vol. 2009, 2009.

[114] Optical Biological Engineering Laboratory (OBEL), "Introduction to oct," http://obel.ee. uwa.edu.au/research/fundamentals/introduction-oct/.

[115] A. Garg, Anterior \& Posterior Segment OCT: Current Technology \& Future Applications. JP Medical Ltd, 2014.

[116] A. Baghaie, Z. Yu, and R. M. D’Souza, "Involuntary eye motion correction in retinal optical coherence tomography: Hardware or software solution?" Medical image analysis, vol. 37, pp. 129-145, 2017.

[117] J. Chhablani, T. Krishnan, V. Sethi, and I. Kozak, "Artifacts in optical coherence tomography," Saudi Journal of Ophthalmology, vol. 28, no. 2, pp. 81-87, 2014.

[118] K. Mohana, D. Bhende, and D. Muna, "Optical coherence tomography: newer techniques, newer machines," Sci J Med Vis Res Found, vol. 2, pp. 75-78, 2015.

[119] B. Baumann, B. Potsaid, M. F. Kraus, J. J. Liu, D. Huang, J. Hornegger, A. E. Cable, J. S. Duker, and J. G. Fujimoto, "Total retinal blood flow measurement with ultrahigh speed swept source/fourier domain oct," Biomedical optics express, vol. 2, no. 6, pp. $1539-1552,2011$.

[120] A. M. Davis, Development of Fourier domain optical coherence tomography for applications in developmental biology. Duke University, 2008.

[121] U. Morgner, W. Drexler, F. Kärtner, X. Li, C. Pitris, E. Ippen, and J. Fujimoto, "Spectroscopic optical coherence tomography," Optics letters, vol. 25, no. 2, pp. 111-113, 2000.

[122] S. Chinn, E. Swanson, and J. Fujimoto, "Optical coherence tomography using a frequency-tunable optical source," Optics letters, vol. 22, no. 5, pp. 340-342, 1997.

[123] F. Lexer, C. K. Hitzenberger, A. Fercher, and M. Kulhavy, "Wavelength-tuning interferometry of intraocular distances," Applied Optics, vol. 36, no. 25, pp. 6548-6553, 1997.

[124] C. L. Shields, S. Kaliki, D. Rojanaporn, S. R. Ferenczy, and J. A. Shields, "Enhanced depth imaging optical coherence tomography of small choroidal melanoma: comparison with choroidal nevus," Archives of Ophthalmology, vol. 130, no. 7, pp. 850-856, 2012.

[125] R. F. Spaide, H. Koizumi, and M. C. Pozonni, "Enhanced depth imaging spectral-domain optical coherence tomography," American journal of ophthalmology, vol. 146, no. 4, pp. 496-500, 2008.

[126] B. Považay, K. Bizheva, B. Hermann, A. Unterhuber, H. Sattmann, A. F. Fercher, W. Drexler, C. Schubert, P. Ahnelt, M. Mei et al., "Enhanced visualization of choroidal vessels using ultrahigh resolution ophthalmic oct at 1050 nm," Optics Express, vol. 11, no. 17, pp. 1980-1986, 2003. 
9. Bibliography

[127] R. F. Spaide, "Enhanced depth imaging optical coherence tomography of retinal pigment epithelial detachment in age-related macular degeneration," American journal of ophthalmology, vol. 147, no. 4, pp. 644-652, 2009.

[128] R. Fitzpatrick, "Representation of waves via complex numbers," http://farside.ph.utexas. edu/teaching/315/Waves/node88.html.

[129] L. Mandel and E. Wolf, Optical coherence and quantum optics. Cambridge university press, 1995.

[130] Biomedical Optics Research Group (BORG), "Fourier domain optical coherence tomography (oct)," http://borg.ensc.sfu.ca/fourieroct.html.

[131] W. Drexler, M. Liu, A. Kumar, T. Kamali, A. Unterhuber, and R. A. Leitgeb, "Optical coherence tomography today: speed, contrast, and multimodality," Journal of biomedical optics, vol. 19, no. 7, p. 071412, 2014.

[132] H. M. Subhash and R. K. Wang, "Optical coherence tomography: technical aspects," in Biomedical Optical Imaging Technologies. Springer, 2013, pp. 163-212.

[133] L. G. Brown, "A survey of image registration techniques," ACM computing surveys (CSUR), vol. 24, no. 4, pp. 325-376, 1992.

[134] J. A. Maintz and M. A. Viergever, "A survey of medical image registration," Medical image analysis, vol. 2, no. 1, pp. 1-36, 1998.

[135] B. Zitova and J. Flusser, "Image registration methods: a survey," Image and vision computing, vol. 21, no. 11, pp. 977-1000, 2003.

[136] B. Fischer and J. Modersitzki, "Ill-posed medicine an introduction to image registration," Inverse Problems, vol. 24, no. 3, p. 034008, 2008.

[137] W. R. Crum, T. Hartkens, and D. Hill, "Non-rigid image registration: theory and practice," The British journal of radiology, vol. 77, no. suppl_2, pp. S140-S153, 2004.

[138] D. L. Hill, P. G. Batchelor, M. Holden, and D. J. Hawkes, "Medical image registration," Physics in medicine \& biology, vol. 46, no. 3, p. R1, 2001.

[139] R. D. Eastman, J. Le Moigne, and N. S. Netanyahu, "Research issues in image registration for remote sensing," in 2007 IEEE Conference on Computer Vision and Pattern Recognition. IEEE, 2007, pp. 1-8.

[140] A. A. Goshtasby, 2-D and 3-D image registration: for medical, remote sensing, and industrial applications. John Wiley \& Sons, 2005.

[141] R. Szeliski et al., "Image alignment and stitching: A tutorial," Foundations and Trends in Computer Graphics and Vision, vol. 2, no. 1, pp. 1-104, 2007.

[142] A. Myronenko, "Non-rigid image registration: Regularization, algorithms and applications," Oregon Health \& Science University, 2010. 
[143] M. Holden, "A review of geometric transformations for nonrigid body registration," IEEE transactions on medical imaging, vol. 27, no. 1, pp. 111-128, 2007.

[144] S. Klein, M. Staring, and J. P. Pluim, "Evaluation of optimization methods for nonrigid medical image registration using mutual information and b-splines," IEEE transactions on image processing, vol. 16, no. 12, pp. 2879-2890, 2007.

[145] S. Wright and J. Nocedal, "Numerical optimization," Springer Science, vol. 35, no. 67-68, p. 7, 1999.

[146] M. J. Powell, "Recent advances in unconstrained optimization," Mathematical Programming, vol. 1, no. 1, pp. 26-57, 1971.

[147] J. J. Waterfall, F. P. Casey, R. N. Gutenkunst, K. S. Brown, C. R. Myers, P. W. Brouwer, V. Elser, and J. P. Sethna, "Sloppy-model universality class and the vandermonde matrix," Physical review letters, vol. 97, no. 15, p. 150601, 2006.

[148] R. N. Gutenkunst, J. J. Waterfall, F. P. Casey, K. S. Brown, C. R. Myers, and J. P. Sethna, "Universally sloppy parameter sensitivities in systems biology models," PLoS computational biology, vol. 3, no. 10, 2007.

[149] J.-P. Thirion, "Fast non-rigid matching of 3d medical images," Rapports de rechercheINRIA, 1995.

[150] J.-P. Thirion, "Image matching as a diffusion process: an analogy with Maxwell's demons," Medical image analysis, vol. 2, no. 3, pp. 243-260, 1998.

[151] R. Sandkühler, C. Jud, S. Andermatt, and P. C. Cattin, "Airlab: Autograd image registration laboratory," arXiv preprint arXiv:1806.09907, 2018.

[152] J. Hadamard, "Sur les problèmes aux dérivées partielles et leur signification physique," Princeton university bulletin, pp. 49-52, 1902.

[153] J. Hadamard, "Lectures on cauchy problem in linear partial differential equations, yale university press, new haven, ct, 1923," Hadamard Lectures on Cauchy's Problem in Linear Partial Differential Equations, 1923.

[154] H. W. Engl, M. Hanke, and A. Neubauer, Regularization of inverse problems. Springer Science \& Business Media, 1996, vol. 375.

[155] A. Roche, G. Malandain, N. Ayache, and S. Prima, "Towards a better comprehension of similarity measures used in medical image registration," in International Conference on Medical Image Computing and Computer-Assisted Intervention. $\quad$ Springer, 1999, pp. 555-566.

[156] J. P. Pluim, J. A. Maintz, and M. A. Viergever, "Mutual information matching and interpolation artifacts," in Medical Imaging 1999: Image Processing, vol. 3661. International Society for Optics and Photonics, 1999, pp. 56-65. 
[157] S. Ourselin, A. Roche, S. Prima, and N. Ayache, "Block matching: A general framework to improve robustness of rigid registration of medical images," in International Conference on Medical Image Computing And Computer-Assisted Intervention. Springer, 2000, pp. $557-566$.

[158] R. Yaakob, A. Aryanfar, A. A. Halin, and N. Sulaiman, "A comparison of different block matching algorithms for motion estimation," Procedia Technology, vol. 11, pp. 199-205, 2013.

[159] D. Rueckert, L. I. Sonoda, C. Hayes, D. L. Hill, M. O. Leach, and D. J. Hawkes, "Nonrigid registration using free-form deformations: application to breast mr images," IEEE transactions on medical imaging, vol. 18, no. 8, pp. 712-721, 1999.

[160] J. A. Schnabel, D. Rueckert, M. Quist, J. M. Blackall, A. D. Castellano-Smith, T. Hartkens, G. P. Penney, W. A. Hall, H. Liu, C. L. Truwit et al., "A generic framework for non-rigid registration based on non-uniform multi-level free-form deformations," in International Conference on Medical Image Computing and Computer-Assisted Intervention. Springer, 2001, pp. 573-581.

[161] I. Bankman, Handbook of medical image processing and analysis. Elsevier, 2008.

[162] P. Čech, A. Andronache, L. Wang, G. Székely, and P. Cattin, "Piecewise rigid multimodal spine registration," Bildverarbeitung für die Medizin 2006, pp. 211-215, 2006.

[163] S. J. Chiu, X. T. Li, P. Nicholas, C. A. Toth, J. A. Izatt, and S. Farsiu, "Automatic segmentation of seven retinal layers in SDOCT images congruent with expert manual segmentation," Optics express, vol. 18, no. 18, pp. 19413-19 428, 2010.

[164] T. Ronchetti, C. Jud, P. M. Maloca, S. Orgül, A. T. Giger, C. Meier, H. P. Scholl, R. K. M. Chun, Q. Liu, C.-H. To et al., "Statistical framework for validation without ground truth of choroidal thickness changes detection," PloS one, vol. 14, no. 6, 2019.

[165] C. Jud, N. Möri, B. Bitterli, and P. C. Cattin, "Bilateral regularization in reproducing kernel hilbert spaces for discontinuity preserving image registration," in International Workshop on Machine Learning in Medical Imaging. Springer, 2016, pp. 10-17.

[166] C. Boote, I. A. Sigal, R. Grytz, Y. Hua, T. D. Nguyen, and M. J. Girard, "Scleral structure and biomechanics," Progress in Retinal and Eye Research, vol. 74, p. 100773, 2020.

[167] I. Oguz, M. D. Abramoff, L. Zhang, K. Lee, E. Z. Zhang, and M. Sonka, "4d graph-based segmentation for reproducible and sensitive choroid quantification from longitudinal oct scans4d choroid segmentation from longitudinal oct scans," Investigative ophthalmology \& visual science, vol. 57, no. 9, pp. OCT621-OCT630, 2016.

[168] R. Chakraborty, S. A. Read, and M. J. Collins, "Diurnal variations in axial length, choroidal thickness, intraocular pressure, and ocular biometrics," Investigative ophthalmology \& visual science, vol. 52, no. 8, pp. 5121-5129, 2011.

[169] V. Grau, A. Mewes, M. Alcaniz, R. Kikinis, and S. K. Warfield, "Improved watershed transform for medical image segmentation using prior information," IEEE transactions on medical imaging, vol. 23, no. 4, pp. 447-458, 2004. 
[170] D. E. Rex, D. W. Shattuck, R. P. Woods, K. L. Narr, E. Luders, K. Rehm, S. E. Stolzner, D. A. Rottenberg, and A. W. Toga, "A meta-algorithm for brain extraction in mri," NeuroImage, vol. 23, no. 2, pp. 625-637, 2004.

[171] G. W. Williams, "Comparing the joint agreement of several raters with another rater," Biometrics, pp. 619-627, 1976.

[172] M. Martin-Fernandez, S. Bouix, L. Ungar, R. W. McCarley, and M. E. Shenton, "Two methods for validating brain tissue classifiers," in International Conference on Medical Image Computing and Computer-Assisted Intervention. Springer, 2005, pp. 515-522.

[173] J. Mazzaferri, L. Beaton, G. Hounye, D. N. Sayah, and S. Costantino, "Open-source algorithm for automatic choroid segmentation of oct volume reconstructions," Scientific Reports, vol. 7, p. 42112, 2017.

[174] R. Gourant and D. Hilbert, "Methods of mathematical physics, vol. ii," Courant, Interscience, 1962.

[175] C. De Boor, C. De Boor, E.-U. Mathématicien, C. De Boor, and C. De Boor, A practical guide to splines. Springer-Verlag New York, 1978, vol. 27.

[176] H. B. Curry and I. J. Schoenberg, "On pólya frequency functions iv: the fundamental spline functions and their limits," Journal d'analyse mathématique, vol. 17, no. 1, pp. 71-107, 1966. 


\section{List of Figures}

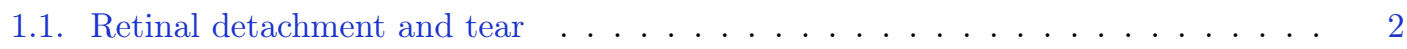

1.2. Interleaved nature of the human eye . . . . . . . . . . . . . . . . 3

2.1. Anatomy of the human eye . . . . . . . . . . . . . . . . . . . . . . . . .

2.2. The three chambers of the human eye . . . . . . . . . . . . . . . . . . . 7

2.3. Choroidal blood flow temperature regulation . . . . . . . . . . . . . . . 8

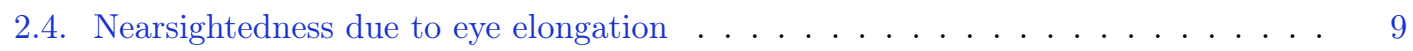

2.5. Optics of the myopic eye . . . . . . . . . . . . . . . . . 10

2.6. Wide field retinal fundus and OCT images . . . . . . . . . . . . . . . . 11

2.7. Retinal detachment as a consequence of pathological myopia . . . . . . . . . . . 13

2.8. Commonly used representations of the thickness of the choroid . . . . . . . . . 14

2.9. The intra- and outer-retinal boundaries . . . . . . . . . . . . . . . . . . . . . . . . . . . . . . .

2.10. Section of the central retina . . . . . . . . . . . . . . . . . 16

2.11. Example of a less recognizable choroid-sclera interface . . . . . . . . . . . . . 17

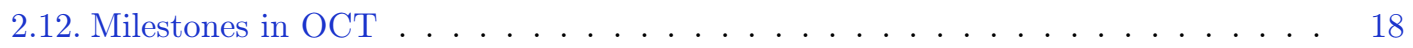

2.13. Comparison of the different imaging modalities . . . . . . . . . . . . . . 19

2.14. Time domain OCT visualized. . . . . . . . . . . . . . . . . . . . . . 20

2.15. The OCT scanning terminology with relative anatomical orientation . . . . . . 21

2.16. The two types of Frequency domain OCT systems . . . . . . . . . . . . . . . 22

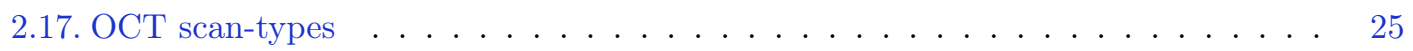

2.18. Signal mirroring in FD-OCT . . . . . . . . . . . . . . . . . . 28

3.1. The principle of image registration . . . . . . . . . . . . . . . . . . . . . . . . . . . . . . . . .

3.2. The B-spline basis functions used . . . . . . . . . . . . . . . . . . . . . . . . . . . . . . . . . . . . .

3.3. The basic idea behind CRAR in $2 \mathrm{D} \ldots \ldots \ldots \ldots$

3.4. Eye-shape adherent regularization . . . . . . . . . . . . . . 37

5.1. Artificial deformation of the choroid . . . . . . . . . . . . . . 59

6.1. Validation of CRAR . . . . . . . . . . . . . . . . . . 78

6.2. Minimal example . . . . . . . . . . . . . . . . . . 80

C.1. Axial and lateral imaging parameters in OCT . . . . . . . . . . . . . . . . 115

C.2. Eye-shape adherent regularization . . . . . . . . . . . . . . . 117 
Appendices 



\section{A. Additional Technical Information}

\section{A.1. Dual-Wavelength System: Hydra-Spectralis}

First of all, we want to emphasize that the optical coherence tomography (OCT)-device which we are presenting was used for most of the image acquisition during these studies. However, our algorithm works for any data format, shape, contrast and texture and is therefore not dependent on a particular OCT-device.

The dual-wavelength SD-OCT (Spectral domain/spectrometer-based) system, called HydraSpectralis, was developed between 2013 and 2017 at the HuCE-optoLab (optical laboratory at the Institute for Human Centered Engineering) at the Bern University of Applied Sciences (BUAS, Berner Fachhochschule) in Switzerland as result of a cooperation project with involvement of the School of Optometry of the Polytechnic University of Hong Kong, the ARTORG (University of Bern), the Department of Biomedical Engineering (University of Basel) and the Zhongshan Ophthalmic Centre in Guangzhou and was funded by the Swiss National Science Foundation (SNSF) and the National Natural Science Foundation of China (NSFC).

The Spectralis system's base hard-, firm- and software originates from Heidelberg Engineering (Germany) and was extended with a second broadband light source that operated at the second ophthalmic NIR window at $1075 \mathrm{~nm}$, a completely reconstructed OCT light path and an additional spectrometer, which was developed at the optoLab. Furthermore, the acquisition, tracking and B-Scan registration firm- and software was rewritten to support the dual synchronous channels. The Spectralis is already a full-fledged commercial and clinically approved integrated $870 \mathrm{~nm}-\mathrm{OCT}$ device, which harbors a coaxial confocal scanning laser ophthalmoscopy (cSLO) that drives an active, real-time eye-tracker.

The scan-head and its light path with the original wavelength of $870 \mathrm{~nm}$ was modified and extended to support the additional wavelength of $1075 \mathrm{~nm}$ for simultaneous imaging at both wavelengths. Longer wavelengths are less likely to be scattered by small particles or inhomogeneities as well as changes of refractive index as found in biological tissue. Henceforth, the weaker $1075 \mathrm{~nm}$ transparency window of water enables to transfer the radiation deeper into and out of biological tissue without inadvertent scatter from the linear propagation. Therefore, signals returning from deeper layers that are hidden under strong scattering tissue, such as the retinal pigment epithelium and the blood-filled choriocapillaris and choroid, correspond better to the actual biological structures, rather than to shadows or other image artifacts like the $870 \mathrm{~nm}$ signals. The choroid can also carry highly scattering melanin at lower concentration than the RPE. It is more likely that signals from deeper layers are less affected and therefore more clearly represent the true internal structure than at the $870 \mathrm{~nm}$ spectrum.

The Hydra-Spectralis system allows to acquire stabilized high-definition cross-sectional images (Brightness-mode or B-scans) of the choroid at both wavelengths. Scattering and absorption reduce the signal with increasing penetration depth. On the other hand, the OCT-signal fea- 


\section{A. Additional Technical Information}

tures a positive and negative copy that is symmetric around the zero delay, which corresponds to the matched length of the object and reference path. If the reference arm is elongated and the zero delay is positioned on the side of the weaker signal stemming from deeper regions, the two effects can balance each other. This inversion of the symmetric signal is also referred to as enhanced depth imaging (EDI) and efficiently counteracts the sensitivity fall-off due to scattering with the sensitivity increase close to the zero delay.

The system does not distinguish between image artifacts, such as the scattered background seen at shorter wavelengths, and real signals, though. Tomograms have been acquired with both channels simultaneously at the same locations in stabilized condition (eye-tracker activated) with up to 100 scans at the same position. Due to the eye-tracker, even less compliant subjects that interrupt the acquisition can participate, since the system re-tracks the original position and re-builds the complete set of scans at the same lateral locations even in long-term follow-up scans. The long sequence acquisition and registered overlapping has multiple implications for the biological information. First, the signal quality is improved due to the $n \times \log (n)$ rule for signal-to-noise improvement for multiple acquisitions. Secondly, the changes in imaging conditions slightly alter the speckle structure of the individual frames that originates from local interference of the light's transverse coherence. Averaging of differently speckled frames thus results in a reduction of the speckle in the final image. Thirdly, small scale tissue variations, e.g. those caused by the heart-beat, are averaged out and build a more reliable baseline for identifying long-term changes of the tissue. 


\section{B. Additional Mathematical Information}

\section{B.1. Mathematical Definitions underlying CRAR}

Definition 1 (Image). Let $\Omega \subset \mathbb{R}^{3}$ be a bounded, connected, open domain. An image is a one-time continuously differentiable function $I: \mathbb{R}^{3} \rightarrow \mathbb{R}$ compactly supported in $\Omega$.

Definition 2 (Transformation). Let $\Omega \subset \mathbb{R}^{3}$ be a domain. A transformation is an invertible and at least twice continuously differentiable function $\psi_{u}: \Omega \rightarrow \mathbb{R}^{3}$ with $\psi_{u}(p)=p+u(p), \forall p \in$ $\Omega$. The function $u: \Omega \rightarrow \mathbb{R}^{3}$ is called displacement. For a differentiable transformation $\psi_{u}$, the Jacobi matrix is denoted by

$$
\nabla \psi_{u}=\left(\partial_{1} \psi_{u}\left|\partial_{2} \psi_{u}\right| \partial_{3} \psi_{u}\right)^{T}=\left(\begin{array}{ccc}
\partial_{1} \psi_{u 1} & \partial_{2} \psi_{u 1} & \partial_{3} \psi_{u 1} \\
\partial_{1} \psi_{u 2} & \partial_{2} \psi_{u 2} & \partial_{3} \psi_{u 2} \\
\partial_{1} \psi_{u 3} & \partial_{2} \psi_{u 3} & \partial_{3} \psi_{u 3}
\end{array}\right)^{T}
$$

As we need to minimize the energy functional $J$ (see Eq. (3.3) on page 34 in Sec. 3.3), the derivatives of the similarity measures $\mathcal{D}^{S S D}$ and $\mathcal{D}^{N C C}$ (see Eq. (3.4) and Eq. (3.5) on page 35 in Sec. 3.3, respectively) are required. In the following, we give important definitions for a comprehensive calculation of their first derivatives.

Definition 3 (Directional Derivative). Let $J: \Omega \subset X \rightarrow Y$ be an operator with Banach spaces $X, Y$ and $\Omega \neq \emptyset . J$ is called directionally differentiable at $p \in \Omega$ if the limit

$$
\partial \mathcal{J}(p, v)=\frac{\partial}{\partial v}(p)=\lim _{\epsilon \rightarrow 0^{+}} \frac{\mathcal{J}(p+\epsilon v)-\mathcal{J}(p)}{\epsilon} \in Y
$$

exists for all $v \in X$. In this case, $\partial \mathcal{J}(p, v)$ is called directional derivative of $J$ in the direction of $v$ and $\partial \mathcal{J}(p, \cdot): X \rightarrow Y, v \mapsto \partial(p, v)$ describes the first variation of $J$ at $p \in \Omega$.

(Note: In our case $X=\mathbb{R}^{3}$ and $Y=\mathcal{U}$. Recall, that $\mathcal{U}=\left\{\psi_{u} \mid \psi_{u}: \Omega \rightarrow \mathbb{R}^{3}, \psi_{u} \in \mathcal{C}^{2}\left(\Omega, \mathbb{R}^{3}\right), \Omega \subset\right.$ $\left.\mathbb{R}^{3}\right\}$ denotes the set of all possible, at least twice continuously differentiable, transformations mapping the domain $\Omega$ into $\mathbb{R}^{3}$, see Sec. 3.2.2 on page 32.)

Definition 4 (Similarity Measure). Let $I_{R}$ and $I_{T}: \Omega \rightarrow \mathbb{R}$ be two images, i.e. $I_{R}, I_{T} \in \mathcal{I}=$ $\left\{I: \Omega \subset \mathbb{R}^{3} \rightarrow \mathbb{R}\right\}$

$$
\mathcal{D}\left(I_{R}, I_{T}, \psi_{u}\right):=\int_{\Omega} \omega(p) \cdot r\left(I_{R}, I_{T} \circ \psi_{u}\right) d p
$$

is the corresponding similarity measure. Here $r: \mathcal{I}^{2} \rightarrow \mathcal{I}$ is a residual or difference function, $\omega: \mathbb{R} \rightarrow \mathbb{R}^{+}$is a weighting function and $\psi_{u} \in C\left(\Omega, \mathbb{R}^{3}\right)$. 


\section{B. Additional Mathematical Information}

Definition 5 (Well-posedness). A mathematical problem is well-posed according to Hadamard's definition, if all the following conditions are fulfilled:

(a) For each datum $I_{T}(p)$ in a given class of functions $\mathcal{I}$ there exists a solution $\psi_{u}$ in a prescribed class $X$ ("existence").

(b) The solution is unique in X ("uniqueness").

(c) The solution of the problem depends continuously on the initial data, i.e. when the error in the original data tends to zero, the induced error in the solution also tends to zero ("continuity") ${ }^{1}$.

\section{B.2. First Variation of $\mathcal{D}^{\mathrm{SSD}}$ and $\mathcal{D}^{\mathrm{NCC}}$}

In order to conduct a minimization of a energy functional, as explained in Sec. Let $\Omega_{j} \subset \mathbb{R}^{2}$ be the $j^{\text {th }}$ slice of size $m \times n$ pixels in a volume stack $\Omega=\bigcup_{j=1}^{S} \Omega_{j} \subset \mathbb{R}^{3}$ of $S$ slices. Utilizing $\mathrm{BM}$ as a reference surface as aforementioned, the volume surrounding the CSI surface is subdivided into partially overlapping 3D cuboidal blocks $\left\{\mathcal{C}_{i}^{s}\right\}$ (where $i=1, \ldots, N$ and $s=1, \ldots, \bar{S}$ ) defined over a regular grid of dimension $\mathcal{N}=N \times \bar{S}$ in the $O_{x y}$ plane, as illustrated in Fig. 3.4 (for additional visual information, see Fig. C.2).

Minimizing $\mathcal{D}^{S S D}$ means applying a fast optimization scheme using derivatives. Therefore, in order to determine the direction of steepest descent, the first variation $\mathcal{D}^{S S D}$ with respect to $u_{i}^{s}$ has to be calculated.

Theorem 1 (First Variation of $\mathcal{D}^{\mathrm{SSD}}$ ). Let $I_{R}$ and $I_{T}: \Omega \subset \mathbb{R}^{3} \rightarrow \mathbb{R}^{+}$be two images. The first variation of $\mathcal{D}^{\mathrm{SSD}}$ is given by

$$
\partial \mathcal{D}^{\mathrm{SSD}}\left(p, u_{i}^{s}\right)=\frac{\partial}{\partial u_{i}^{s}} \mathcal{D}^{\mathrm{SSD}}(p)=2 \sum_{s=1}^{\bar{S}} \sum_{i=1}^{N} \int_{p \in \hat{\mathcal{C}}_{i}^{s}}\left\|I_{T}\left(p+u_{i}^{s}(p)\right)-I_{R}(p)\right\| \frac{\partial}{\partial u_{i}^{s}} I_{T}\left(p+u_{i}^{s}(p)\right) d p,
$$

where $\hat{\mathcal{C}}_{i}^{s}$ denotes the overlapping volume between reference and transformed template image and $p$ is the point position in block $\hat{\mathcal{C}}_{i}^{s} \subset \Omega$.

Proof. Using the linearity of the differential operator and the chain rule, we calculate the partial derivative of the functional $\mathcal{D}^{\mathrm{SSD}}$ at the point $p \in \hat{\mathcal{C}}_{i}^{s}$ with respect to the parameter $u_{i}^{s}$ :

$$
\begin{aligned}
\frac{\partial}{\partial u_{i}^{s}} \mathcal{D}^{\mathrm{SSD}}(p) & =\frac{\partial}{\partial u_{i}^{s}} \sum_{s=1}^{\bar{S}} \sum_{i=1}^{N} \int_{p \in \hat{\mathcal{C}}_{i}^{s}}\left\|I_{T}\left(p+u_{i}^{s}(p)\right)-I_{R}(p)\right\|^{2} d p \\
& =\sum_{s=1}^{\bar{S}} \sum_{i=1}^{N} \int_{p \in \hat{\mathcal{C}}_{i}^{s}} \frac{\partial}{\partial u_{i}^{s}}\left\|I_{T}\left(p+u_{i}^{s}(p)\right)-I_{R}(p)\right\|^{2} d p \\
& =2 \sum_{s=1}^{\bar{S}} \sum_{i=1}^{N} \int_{p \in \hat{\mathcal{C}}_{i}^{s}} \| I_{T}\left(\left(p+u_{i}^{s}(p)\right)-I_{R}(p) \| \frac{\partial}{\partial u_{i}^{s}} I_{T}\left(p+u_{i}^{s}(p)\right) d p\right.
\end{aligned}
$$

\footnotetext{
${ }^{1}$ Continuity should be seen in the light of that of stability or robustness of the solution [174]. However, although it is a necessary condition for stability, on its own it is not sufficient.
} 
Theorem 2 (First Variation of $\mathcal{D}^{\mathrm{NCC}}$ ). The first variation of $\mathcal{D}^{\mathrm{NCC}}$ is given by

$$
\partial \mathcal{D}^{\mathrm{NCC}}\left(p, u_{i}^{s}\right)=\sum_{s=1}^{\bar{S}} \sum_{i=1}^{N} \frac{\sum_{p \in \hat{\mathcal{C}}_{i}^{S}} \frac{\partial}{\partial u_{i}^{s}} A_{T} B_{R} \sqrt{\sum_{p \in \hat{\mathcal{C}}_{i}^{s}} A_{T}^{2} \sum_{p \in \hat{\mathcal{C}}_{i}^{s}} B_{R}^{2}}-\sum_{p \in \hat{\mathcal{C}}_{i}^{s}} A_{T} B_{R} \frac{\partial}{\partial u_{i}^{s}} \sqrt{\sum_{p \in \hat{\mathcal{C}}_{i}^{s}} A_{T}^{2} \sum_{p \in \hat{\mathcal{C}}_{i}^{s}} B_{R}^{2}}}{\left(\sum_{p \in \hat{\mathcal{C}}_{i}^{s}} A_{T}^{2}\right)\left(\sum_{p \in \hat{\mathcal{C}}_{i}^{s}} B_{R}^{2}\right)}
$$

where $A_{T}=I_{T}\left(p+u_{i}^{s}(p)\right)-\mu_{T}$ and $B_{R}=I_{R}(p)-\mu_{R}$. Besides, $\mu_{R}$ and $\mu_{T}$ are the average intensities of $I_{R}$ and $I_{T}$ respectively, while $\hat{\mathcal{C}}_{i}^{s}$ denotes the overlapping volume between reference and transformed template image and $p$ is the point position in block $\hat{\mathcal{C}}_{i}^{s} \subset \Omega$.

Proof. Using the linearity of the differential operator, the quotient and the chain rule, we calculate the partial derivative of the functional $\mathcal{D}^{\mathrm{NCC}}$ at the point $p \in \hat{\mathcal{C}}_{i}^{s}$ with respect to the parameter $u_{i}^{s}$ :

$$
\begin{aligned}
& \frac{\partial}{\partial u_{i}^{s}} \mathcal{D}^{\mathrm{NCC}}\left(p, u_{i}^{s}\right)=-\frac{\partial}{\partial u_{i}^{s}} \sum_{s=1}^{\bar{S}} \sum_{i=1}^{N} \int_{p \in \hat{\mathcal{C}}_{i}^{s}} \frac{\sum_{p \in \hat{\mathcal{C}}_{i}^{s}}\left[I_{T} \circ \psi_{u_{i}^{s}}(p)-\mu_{T}\right]\left[I_{R}(p)-\mu_{R}\right]}{\sqrt{\sum_{p \in \hat{\mathcal{C}}_{i}^{s}}\left[I_{T} \circ \psi_{u_{i}^{s}}(p)-\mu_{T}\right]^{2}} \sqrt{\sum_{p \in \hat{\mathcal{C}}_{i}^{s}}\left[I_{R}(p)-\mu_{R}\right]^{2}}} \\
& =-\sum_{s=1}^{\bar{S}} \sum_{i=1}^{N} \frac{\partial}{\partial u_{i}^{s}} \frac{\sum_{p \in \hat{\mathcal{C}}_{i}^{s}}\left[I_{T}\left(p+u_{i}^{s}(p)\right)-\mu_{T}\right]\left[I_{R}(p)-\mu_{R}\right]}{\sqrt{\sum_{p \in \hat{\mathcal{C}}_{i}^{s}}\left[I_{T}\left(p+u_{i}^{s}(p)\right)-\mu_{T}\right]^{2}} \sqrt{\sum_{p \in \hat{\mathcal{C}}_{i}^{s}}\left[I_{R}(p)-\mu_{R}\right]^{2}}} \\
& =-\sum_{s=1}^{\bar{S}} \sum_{i=1}^{N} \frac{\partial}{\partial u_{i}^{s}} \frac{\sum_{p \in \hat{\mathcal{C}}_{i}^{s}} A_{T} B_{R}}{\sqrt{\sum_{p \in \hat{\mathcal{C}}_{i}^{s}} A_{T}^{2} \sum_{p \in \hat{\mathcal{C}}_{i}^{s}} B_{R}^{2}}} \\
& =-\sum_{s=1}^{\bar{S}} \sum_{i=1}^{N} \frac{\sum_{p \in \hat{\mathcal{C}}_{i}^{s}} \frac{\partial}{\partial u_{i}^{s}} A_{T} B_{R} \sqrt{\sum_{p \in \hat{\mathcal{C}}_{i}^{s}} A_{T}^{2} \sum_{p \in \hat{\mathcal{C}}_{i}^{s}} B_{R}^{2}}-\sum_{p \in \hat{\mathcal{C}}_{i}^{s}} A_{T} B_{R} \frac{\partial}{\partial u_{i}^{s}} \sqrt{\sum_{p \in \hat{\mathcal{C}}_{i}^{s}} A_{T}^{2} \sum_{p \in \hat{\mathcal{C}}_{i}^{s}} B_{R}^{2}}}{\left(\sum_{p \in \hat{\mathcal{C}}_{i}^{s}} A_{T}^{2}\right)\left(\sum_{p \in \hat{\mathcal{C}}_{i}^{s}} B_{R}^{2}\right)}
\end{aligned}
$$

For the detailed calculation, please take note of the following:

$A_{T}=I_{T}\left(p+u_{i}^{s}(p)\right)-\mu_{T}$ and $B_{R}=I_{R}(p)-\mu_{R}$

$\frac{\partial}{\partial u_{i}^{s}} \sum_{p \in \hat{\mathcal{C}}_{i}^{s}} A_{T}=\frac{\partial}{\partial u_{i}^{s}} \sum_{p \in \hat{\mathcal{C}}_{i}^{s}}\left(I_{T}\left(p+u_{i}^{s}(p)\right)-\mu_{T}\right)=\sum_{p \in \hat{\mathcal{C}}_{i}^{s}} \frac{\partial}{\partial u_{i}^{s}} I_{T}\left(p+u_{i}^{s}(p)\right)$

$\frac{\partial}{\partial u_{i}^{s}} \sum_{p \in \hat{\mathcal{C}}_{i}^{s}} A_{T}^{2}=\frac{\partial}{\partial u_{i}^{s}} \sum_{p \in \hat{\mathcal{C}}_{i}^{s}}\left(I_{T}\left(p+u_{i}^{s}(p)\right)-\mu_{T}\right)^{2}=2 \sum_{p \in \hat{\mathcal{C}}_{i}^{s}} \frac{\partial}{\partial u_{i}^{s}} I_{T}\left(p+u_{i}^{s}(p)\right) \cdot\left(I_{T}\left(p+u_{i}^{s}(p)\right)-\mu_{T}\right)$

$\frac{\partial}{\partial u_{i}^{s}} \sum_{p \in \hat{\mathcal{C}}_{i}^{s}} B_{R}=\frac{\partial}{\partial u_{i}^{s}} \sum_{p \in \hat{\mathcal{C}}_{i}^{s}}\left(I_{R}(p)-\mu_{R}\right)=0$

$\frac{\partial}{\partial u_{i}^{s}} \sum_{p \in \hat{\mathcal{C}}_{i}^{s}} B_{R}^{2}=\frac{\partial}{\partial u_{i}^{s}} \sum_{p \in \hat{\mathcal{C}}_{i}^{s}}\left(I_{R}(p)-\mu_{R}\right)^{2}=0$

$\frac{\partial}{\partial u_{i}^{s}} \sqrt{\sum_{p \in \hat{\mathcal{C}}_{i}^{s}} A_{T}^{2} \sum_{p \in \hat{\mathcal{C}}_{i}^{s}} B_{R}^{2}}=\frac{\sum_{p \in \hat{\mathcal{C}}_{i}^{s}} B_{R}^{2} \sum_{p \in \hat{\mathcal{C}}_{i}^{s}} \frac{\partial}{\partial u_{i}^{s}} I_{T}\left(p+u_{i}^{s}(p)\right) \cdot\left(I_{T}\left(p+u_{i}^{s}(p)\right)-\mu_{T}\right)}{\sqrt{\sum_{p \in \hat{\mathcal{C}}_{i}^{s}} A_{T}^{2} \sum_{p \in \hat{\mathcal{C}}_{i}^{s}} B_{R}^{2}}}$

\section{B.3. B-Splines}

Definition 6 (Spline function). Let $\left[x_{i-k}, x_{i}\right]$ be a closed interval subdivided into $N$ distinct equidistant cells with center points $x_{i}=(2 i-1) \frac{\omega}{2}, i=1, \ldots, N$, where $\omega=\left|x_{i+1}-x_{i}\right|$. A spline $s_{k}(x)$ of order $k$ is a function which

(i) in each open interval $\left(x_{i}, x_{i+1}\right)$ is a polynomial of degree $k$, and 


\section{B. Additional Mathematical Information}

(ii) has $(k-1)$ continuous derivatives in the open interval $\left(x_{1}, x_{N}\right)$.

For each fixed set of knots as in Def. 6, the class of such splines is a linear space of functions with $(N+k)$ free parameters. A basis for this linear space is provided by "Schoenberg's Bsplines", or "Basic splines" [175, 176].

Definition 7 (B-splines definitions). Let $\left[x_{i-k}, x_{i}\right]$ be a closed interval subdivided into $N$ distinct equidistant cells with center points $x_{i}=(2 i-1) \frac{\omega}{2}, i=1, \ldots, N$, where $\omega=\left|x_{i+1}-x_{i}\right|$. A B-spline $\beta_{k}(x)$ of order $k$ is a non-negative spline function (as in Def. 6)

$$
\beta_{k}(t)=\left[x_{i-k}, x_{i}\right]\left(x_{i}-t\right)_{+}^{k-1}, \text { where }\left(x_{i}-t\right)_{+}^{k-1}=(\max \{x-t, 0\})^{k-1}
$$

which vanishes outside of $\left[x_{i-k}, x_{i}\right]$.

To obtain a basis of B-splines, we adjoin $k$ additional knots to the ends of the interval $\left[x_{1}, x_{\mathcal{N}}\right]$ in the form $x_{1-k} \leq x_{2-k} \leq \ldots \leq x_{1}$ and $x_{N} \leq x_{N+1} \ldots \leq x_{N+k}$, respectively. According to [176], there exists a unique vector of coefficients $u=\left(u_{1}, \ldots, u_{N+k)}\right.$ that

$$
s_{k}(t)=\sum_{i=1}^{\mathcal{N}+k} u_{i} B_{i}(t) .
$$

Theorem 3 (Holladay Theorem). Let $\left[x_{i-1}, x_{i}\right]$ be the $i$-th cell as in Def. 6 . Furthermore, let $\left\{z_{i}\right\}_{k=1}^{N}$ be a set of real numbers. Following, among all functions $f(x)$ with a continuous second derivative on $\left[x_{0}, x_{N}\right]$ and such that $f\left(x_{i}\right)=z_{i} \forall i \in\{1, \ldots, N\}$, the spline function $s_{k}$ with the knots at $x_{k}$ and with $s^{\prime \prime}\left(x_{1}\right)=s^{\prime \prime}\left(x_{N}\right)=0$ minimizes the integral

$$
\int_{x_{1}}^{x_{N}}\left(f^{\prime \prime}(x)\right)^{2} d x
$$

In addition, the spline function $s_{k}(x)$ is defined to be composed of cubic polynomials in each subinterval $\left[x_{i-1}, x_{i}\right]$, and satisfies the following conditions:

$$
\begin{aligned}
& \text { i) } s^{\prime}\left(x_{k}^{+}\right):=\lim _{x \rightarrow x_{k}^{+}} s^{\prime}\left(x_{k}\right)=\lim _{x \rightarrow x_{k}^{-}} s^{\prime}\left(x_{k}\right)=s^{\prime}\left(x_{k}^{-}\right), k \in\{2, \ldots, N-1\} \\
& \text { ii) } s^{\prime \prime}\left(x_{k}^{+}\right):=\lim _{x \rightarrow x_{k}^{+}} s^{\prime \prime}\left(x_{k}\right)=\lim _{x \rightarrow x_{k}^{-}} s^{\prime \prime}\left(x_{k}\right)=s^{\prime \prime}\left(x_{k}^{-}\right), k \in\{2, \ldots, N-1\}
\end{aligned}
$$

In other words, a spline $s_{k}$ can be approximated by the shift and superposition of third order basis functions, provided that their derivatives are zero at the boundary points. 


\section{Additional Visual Information}

\section{C.1. Illustrations Technical Background}

Axial and lateral imaging parameters in OCT and schematic illustration of a 3Dvolume scan (in addition to Fig. 2.15 and 2.17).

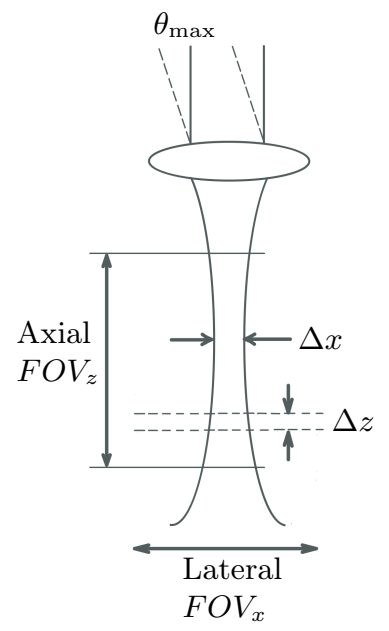

(a)

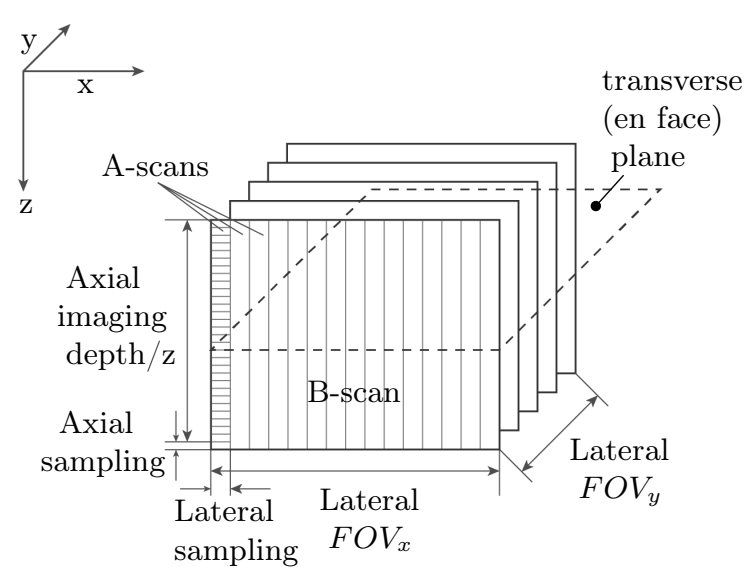

(b)

Figure C.1.: Imaging parameters and scanning terminology in OCT.

(a) Axial and lateral imaging parameters in OCT depending on the focusing of the sample arm beam. $F O V_{z}$ and $F O V_{x}$ denote the axial and lateral field of view, while $\Delta z$ and $\Delta x$ are the axial and lateral resolution, respectively. (b) The set-up of a C-scan. An A-scan is produced along the $z$ /axial-direction. When the $z$ - is combined with the $x$ /lateral-direction a $2 \mathrm{D}$ cross-section, called B-scan, is the result. Adding the $y /$ transverse-direction generates a 3D-volume stack of parallel B-scans, defined as a C-scan. (Figure adaptet from [103,104].)

As described in Sec. 2.2.2, the image information in an A-scan, a depth-resolved reflectivity profile (along the axial $/ z$-direction), is generated from the detected intensity spectrum by applying the inverse Fourier transform [131]. Due to the Fourier relation between the time and frequency domain, a spectral decomposition of the detected interferogram happens instantaneously without moving the reference arm (unlike the procedure in TD-OCT).

The lateral and axial resolutions, see Fig. C.1, are the key factors in all application fields of OCT. Especially retinal and choroidal imaging require a high axial resolution to allow deep penetration [132]. 


\section{Additional Visual Information}

The term "axial/ lateral resolution" (unit: $\mu \mathrm{m}$ ) is often inappropriately used to indicate the "axial/lateral sampling" (unit: $\mu \mathrm{m} / \mathrm{pixel}$ ), which defines how many micrometer per pixels are imaged and provide the axial/lateral scaling of the scan. On the other side, the resolution defines the minimal distance between structures at which they can still be distinguished in an OCT B-scan [104].

Assuming a Gaussian spectrum, the axial resolution $\Delta z$ is related to the coherence length of the light source bandwidth, stated both in wavenumber and wavelength terms as

$$
\Delta z=\frac{2 \sqrt{\ln 2}}{\Delta k_{\mathrm{FWHM}}}=\frac{2 \ln 2}{\pi} \frac{\lambda_{0}^{2}}{\Delta \lambda_{\mathrm{FWHM}}} \approx 0.44 \frac{\lambda_{0}^{2}}{\Delta \lambda_{\mathrm{FWHM}}},
$$

where $\lambda_{0}=2 \pi / k_{0}$ is the center wavelength of the light source and $\Delta \lambda_{\mathrm{FWHM}}$ denotes the bandwidth, defined as the "Full Width at Half Maximum" ${ }^{1}$ of its wavelength spectrum, according to the relationship

$$
\Delta k_{\mathrm{FWHM}}=\frac{\pi}{\sqrt{\ln 2}} \frac{\Delta \lambda_{\mathrm{FWHM}}}{\lambda_{0}^{2}}=\frac{2 \sqrt{\ln 2}}{\Delta z} .
$$

As shown in Eq. (C.1), the axial resolution is inversely proportional to the spectral width of the light source. To achieve an high axial resolution, a broadband light source is therefore necessary [103].

The lateral resolution is defined by the beam diameter at half maximum, according to

$$
\Delta x=\frac{\sqrt{2 \ln 2}}{\pi} \frac{\lambda_{0}}{\mathrm{NA}} \approx 0.37 \frac{\lambda_{0}}{\mathrm{NA}},
$$

where NA denotes the numerical aperture of an optical system (in this case the human eye) and is defined as the range of angles over which the system can accept or emit light. (The NA can also be considered as a measure of the optical resolution in a diffraction-limited system.) In the case of the human eye, it is given by

$$
\mathrm{NA}=n_{\text {vitr. }} \frac{D}{2 f_{p}},
$$

where $n=1.336$ is the refractive index of the vitreous, $D$ the pupil diameter $(\approx 3 \mathrm{~mm}$ for undilated pupils, in the range of $6-8 \mathrm{~mm}$ for dilated pupils), and $f_{p}(=22.3 \mathrm{~mm})$ the posterior focal length of the emmetropic eye. In order to achieve a high lateral resolution $\Delta x$, a large NA is required [132], see Eq. (C.3).

As illustrated in Fig. C.1, the lateral field of view $\left(F O V_{x}\right)$ is defined by

$$
F O V_{x}=2 \cdot f \cdot \theta_{\max }
$$

where $\theta_{\max }$ denotes the maximum scan angle, $f$ is the focal length of the objective lens [103].

\footnotetext{
${ }^{1}$ The full width at half maximum (FWHM) is the width of a line shape at half of its maximum amplitude.

The FWHM is vital to define resolution. For Gaussian line shapes, it is about 2.4 standard deviations.
} 
The FWHM power of the confocal axial response function is defined as the axial field of view $\left(F O V_{z}\right)$ according to

$$
F O V_{z}=\frac{0.565 \lambda}{\sin ^{2}(0.5 \arcsin (N A))}
$$

As shown in Eq. (C.3), lateral resolution in OCT, limited by geometrical optics constraints of the sample arm design, is independent of the bandwidth of the light source and, thus of the axial resolution. Unlike in conventional microscopy, OCT therefore allows a high axial resolution to be achieved despite the limited diameter of the eye pupil. (For a comprehensive and detailed explanation the reader is referred to [103,104,130-132].)

\section{C.2. Illustrations Mathematical Background}

\section{Eye-shape adherent regularization (alternative illustration to Fig. 3.4).}

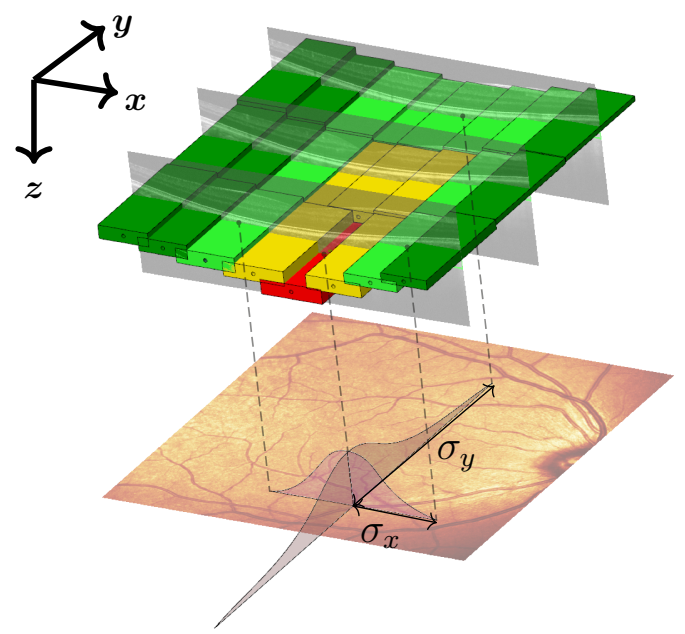

(a)

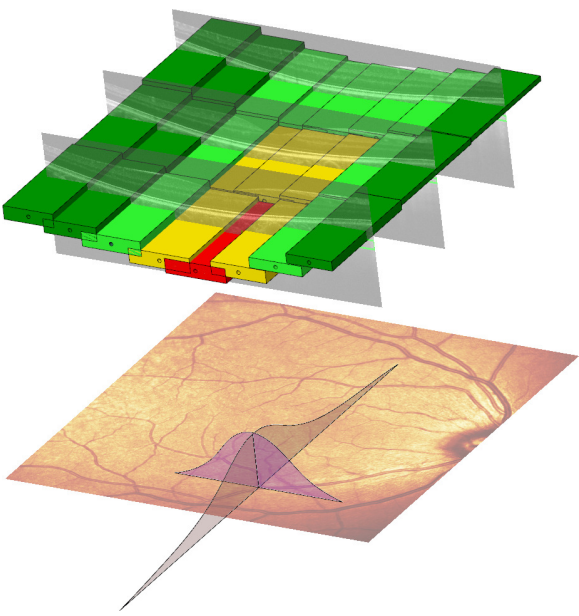

(b)

Figure C.2.: Eye-shape adherent regularization.

(a) Block matching leaves some blocks mismatched (red). Underneath both 1D B-spline kernels with their support $2 \sigma_{x}$ and $2 \sigma_{y}$ are visualized. The regularization corrects the position of the mismatched block in the $3 \mathrm{D}$ registration. This movement is counteracted by the directly neighboring blocks (yellow), which has a smoothing effect. Blocks that are further away (light green) do not have such strong influence. (b) The smoothed result after eye shape adherent regularization. 



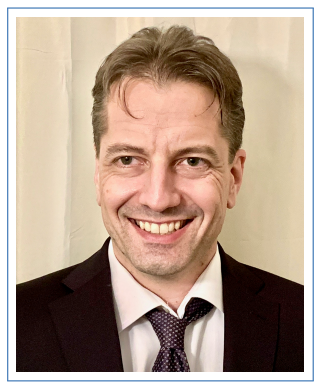

Tiziano Ronchetti

Curriculum Vitae

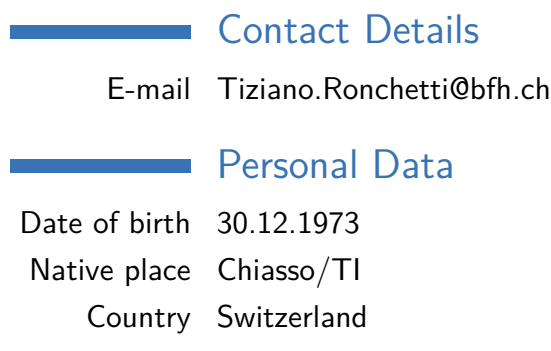

\section{Education}

2015-2020 PhD in Biomedical Engineering, CIAN/DBE, Medical Faculty, University of Basel. Topic: Detecting Early Choroidal Changes Using Piecewise Rigid Image Registration and Eye-Shape Adherent Regularization. Graduation on September 2020. Primary Advisor: Prof. Dr. Selim Orgül. Secondary Advisor: Prof. Dr. Philippe C. Cattin. External Expert: Prof. Dr. Dr. Daniel Barthelmes. Further Experts: Dr. Christoph Jud, Dr. Boris Považay and Dr. Peter Maloca.

1997-2001 High school teacher diploma in Mathematics and Chemistry, Höheres Lehramt, University of Bern. Completion of teacher-training in summer 1998. Thesis and main exam in fall 2001. Topic: Computer simulation in Quantum Chemistry. Supervisor: Prof. Dr. Günther Baars.

1992-1997 Master of Science (Lic. phil.-nat.) in Mathematics (major) and Chemistry (minor), University of Bern. Completion with Diploma of phil.-nat. faculty on 26.06.1997. Master Thesis (Diplomarbeit): Classification Configuration Spaces of Polygonal Linkages. Supervisor: Prof. Dr. Peter Mani-Levitska.

1989-1992 Matura (type B), Literaturgymnasium, Liceo Lugano 1, Lugano. Main focus: French, German and Latin (and the corresponding literature). Graduation on 30.06.1992. 
Curriculum Vitae

\section{Teaching Experience}

since 2007 Lectures in Mathematics, Bern University of Applied Sciences, Department MNG, Biel, Bern and Burgdorf.

since 2003 Teaching in Mathematics, Berufsmaturitätsschule BMS/GIBB.

1998-2003 Lectures in Mathematics, ISBE-HTA, Bern.

1993-1998 High school teaching in Mathematics, Gymnasium Langenthal, Neufeld Gymnasium and Freies Gymnasium Bern.

\section{PhD Research Project}

SNSF Project 320030-146021 "Characterisation of choroidal changes in children and its temporal response to optical defocus", an international cooperation between the University of Applied Sciences of Biel (project center), the Department of Biomedical Engineering of the University of Basel (academic supervision), Department of Ophthalmology (clinical support), Guangzhou and Hong Kong (clinical partners). The persons responsible are Prof. Christoph Meier (head of the HuCE-OptoLab of the Bern University of Applied Sciences in Biel) and the principal investigator Dr. Boris Považay.

\section{Publications}

○ T. Ronchetti, P. Maloca, C. Meier, S. Orgül, C. Jud, P. Hasler, B. Považay, and P. C. Cattin, "Intensity-based choroidal registration using regularized block matching," in Proceedings of the Ophthalmic Medical Image Analysis Third International Workshop (OMIA), 2016, pp. 33-40.

○ T. Ronchetti, P. Maloca, C. Jud, C. Meier, S. Orgül, H. P. Scholl, B. Považay, and P. C. Cattin, "Detecting early choroidal changes using piecewise rigid image registration and eye-shape adherent regularization," in Fetal, Infant and Ophthalmic Medical Image Analysis. Springer, 2017, pp. 92-100.

$\circ$ T. Ronchetti, C. Jud, P. M. Maloca, S. Orgül, A. T. Giger, C. Meier, H. P. Scholl, R. K. M. Chun, Q. Liu, C.-H. To, B. Považay, and P. C. Cattin, "Statistical framework for validation without ground truth of choroidal thickness changes detection," PloS one, vol. 14, no. 6, p. e0218776, 2019.

○ T. Ronchetti, P. Maloca, E. R. de Carvalho, T. F. Heeren, K. Balaskas, A. Tufail, C. Egan, M. Okada, S. Orgül, C. Jud, and P. C. Cattin, "Feasibility study of subfoveal choroidal thickness changes in spectral-domain optical coherence tomography measurements of macular telangiectasia type 2," in Computational Pathology and Ophthalmic Medical Image Analysis. Springer, 2018, pp. 303-309.

\section{Research Interests}

- Medical image analysis with a particular focus on kernel methods for image registration.

- Deep Learning methods for image segmentation and registration. 


\section{Languages}

- Italian, mother tongue.

- German, very fluent (written and orally).

o French, very fluent (written and orally).

○ English, fluent (written and orally).

- Spanish, fluent (written and orally).

o Portuguese, somewhat fluent (orally). 